\title{
Non-small cell lung cancer
}

\author{
Citation for published version (APA):
}

Sosa Iglesias, V. (2018). Non-small cell lung cancer: taking it down a NOTCH : NOTCH inhibitie in nietkleincellig long carcinoom . [Doctoral Thesis, Maastricht University]. Gildeprint en Universitaire Pers Maastricht. https://doi.org/10.26481/dis.20180703vs

\section{Document status and date:}

Published: 01/01/2018

DOI:

10.26481/dis.20180703vs

Document Version:

Publisher's PDF, also known as Version of record

\section{Please check the document version of this publication:}

- A submitted manuscript is the version of the article upon submission and before peer-review. There can be important differences between the submitted version and the official published version of record.

People interested in the research are advised to contact the author for the final version of the publication, or visit the DOI to the publisher's website.

- The final author version and the galley proof are versions of the publication after peer review.

- The final published version features the final layout of the paper including the volume, issue and page numbers.

Link to publication

\footnotetext{
General rights rights.

- You may freely distribute the URL identifying the publication in the public portal. please follow below link for the End User Agreement:

www.umlib.nl/taverne-license

Take down policy

If you believe that this document breaches copyright please contact us at:

repository@maastrichtuniversity.nl

providing details and we will investigate your claim.
}

Copyright and moral rights for the publications made accessible in the public portal are retained by the authors and/or other copyright owners and it is a condition of accessing publications that users recognise and abide by the legal requirements associated with these

- Users may download and print one copy of any publication from the public portal for the purpose of private study or research.

- You may not further distribute the material or use it for any profit-making activity or commercial gain

If the publication is distributed under the terms of Article $25 \mathrm{fa}$ of the Dutch Copyright Act, indicated by the "Taverne" license above, 


\section{Non-small cell lung cancer: taking it down a NOTCH}

\section{NOTCH inhibitie in niet-kleincellig long carcinoom}

by

\section{Venus Sosa Iglesias}

Department of Radiotherapy

GROW - School for Oncology and Developmental Biology

Maastricht University

Maastricht, The Netherlands 
(C) Venus Sosa Iglesias, 2018. Maastricht, The Netherlands

All rights reserved. No part of this publication may be reproduced, stored in a retrieval system, or transmitted, in any form or by any means without written permission of the author or the publisher holding the copyright of the published articles.

Financial support for the execution of this thesis was provided by Worldwide Cancer Research. Madras House, St Andrews, Fife, Scotland, KY16 9EH. Registered in Scotland, No 152991, Charity No SC022918

Printing costs were kindly provided by the University of Maastricht and MaastRO (Maastricht Radiation Oncology) Clinic, in the Netherlands.

Cover design: Venus Sosa Iglesias, using www.canva.com

"Scientists in unison working together to protect human lungs."

"When I walk along with two others, from at least one I will be able to learn." (Confucius) "Do your little bit of good where you are; it's those little bits of good put together that overwhelm the world." (Desmond Tutu)

Hand photos from Arjan Groot, Carolien Wansleeben, Damiënne Marcus, Eloy Moreno Roig, Jonathan Ient, Kranthi Panth Marella, Lorena Giuranno, Lotte Schyns, Lydie Barbeau, Marijke Zonneveld, Marike van Gisbergen, Mckneil Tupaki Rongokavea, Clyde Drexlar Misi, Sanam Radhika, Ronald Rolland, Alpheus Bana, Nanda Parvathaneni, Rianne Biemans, Sanaz Yahyanejad, Veronica Olivo Pimentel, and Venus Sosa Iglesias

Printing: Gildeprint (https://www.gildeprint.nl/) 


\section{Non-small cell lung cancer: taking it down a NOTCH}

\section{NOTCH inhibitie in niet-kleincellig long carcinoom}

by

\section{Venus Sosa Iglesias}

Dissertation to obtain the degree of

Doctor at Maastricht University, on the authority of the

Rector Magnificus Prof.dr. Rianne M. Letschert, in accordance with the decision of the Board of Deans, to be defended in public on

Tuesday, July $3^{\text {rd }} 2018$ at 10 hours at Maastricht University 


\title{
Promoter:
}

Prof. Marc Vooijs, PhD

\section{Co-promoters:}

Assist. Prof. Jan Theys, PhD

Assoc. Prof. Ludwig Dubois, PhD

\begin{abstract}
Assessment Committee:
Prof. Dirk De Ruysscher, MD, PhD (chair) (MaastRO Clinic)

Prof. Boris Kramer, MD, PhD (Maastricht University Medical Center)

Prof. Frans Ramaekers, PhD (Maastricht University Medical Center)

Prof. Jan Bussink, MD, PhD (Radboud University Medical Center)

Pierre Close, $\mathrm{PhD}$ (University of Liège)
\end{abstract}




\section{TABLE OF CONTENTS}

Chapter 1: $\quad$ Introduction to NOTCH signaling in cancer

Chapter 2: $\quad$ Drug resistance in non-small cell lung cancer: a potential for NOTCH targeting?

Chapter 3: $\quad$ NOTCH inhibition enhances synergistically the efficacy of conventional chemotherapeutics and chemoradiation in non-squamous NSCLC cell lines

Chapter 4: $\quad$ NOTCH inhibition to enhance cisplatin and radiation treatment in human NSCLC xenografts

Chapter 5: A novel clinically relevant orthotopic NSCLC model for image-guided small animal radiotherapy platforms

Chapter 6: NOTCH blockade combined with radiation therapy and temozolomide prolongs survival of orthotopic glioblastoma.

Chapter 7: $\quad$ General discussion and future perspectives
Addendum:
Summary
Valorization
Acknowledgements
Curriculum Vitae
List of publications 


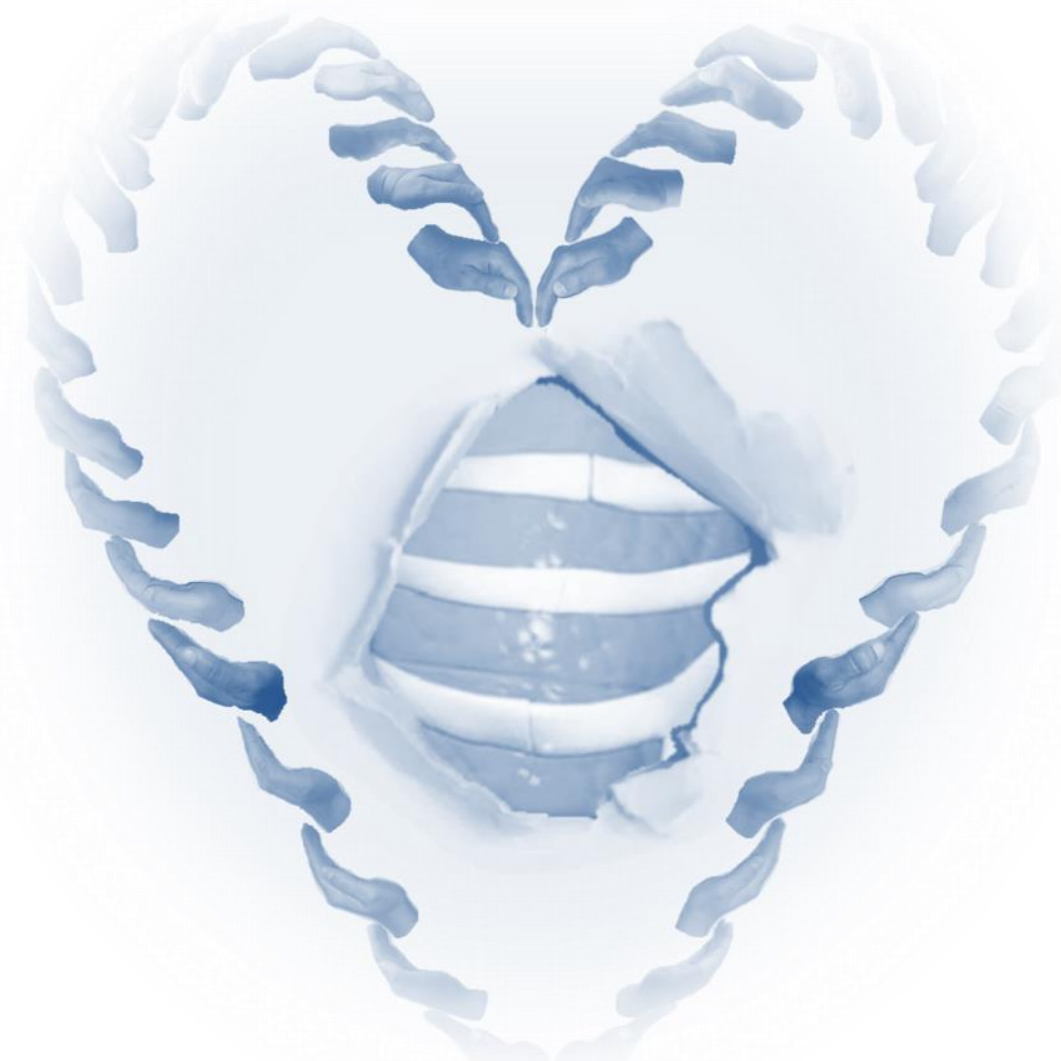




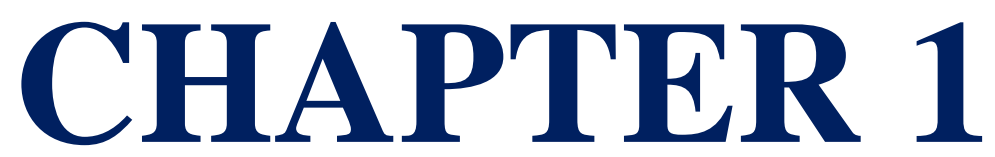

Introduction to NOTCH signaling in cancer 


\section{TABLE OF CONTENTS}

INTRODUCTION

TUMOR RESISTANCE

LUNG CANCER

GLIOBLASTOMA (GBM)

Stem Cell PathWays: TARgets For Precision Therapy

NOTCH: A Historical PERSPECTIVE

NOTCH SIGNALING

NOTCH ENDOCYTOSIS

NOTCH IN NORMAL PHYSiOlogy

NOTCH IN LUNG AND BRAIN CANCERS

DUALITY OF NOTCH ACTIVITY IN CANCER

NOTCH AS AN ONCOGENE

NOTCH AS TUMOR SUPPRESSOR

NOTCH-BASED THERAPEUTIC INTERVENTIONS 


\section{Introduction}

Cancer presents a major health and economic problem worldwide. In 2015, about 8.8 million people died of cancer worldwide ( 1 out of 6 deaths is due to cancer). By 2030, it is expected that there will be 23.6 million new cases of cancer each year. Lung, liver, colorectal, stomach, and breast cancers are the most frequent cancerrelated deaths in decreasing order (www.who.int). There are over 100 different types of cancer and symptoms vary with the tissue of origin. In 2000, Hanahan and Weinberg published a seminal review on the origins of cancer and identified six capabilities every cancer cell has to acquire to transform from normal into an invasive cancer [1]. The six hallmarks that are common to most cancer types are: "sustaining proliferative signaling", "evading growth suppressors", "activating invasion and metastasis", "enabling replicative immortality", "inducing angiogenesis", and finally, "resisting cell death". They revisited these hallmarks in 2011 and identified "tumor-promoting inflammation", "avoiding immune destruction", "deregulating cellular energetics", and "genome instability and mutation" as "enablers' of the aforementioned six acquired capabilities [2]. This discovery has led to the development of small molecules and antibody-based approaches to target the hallmarks cancer, which include: 1) targeting proliferative signaling using neutralizing antibodies against EGFR or HER2 in cancers overexpressing these oncogenic driver mutations [3, 4], 2) evading growth suppressors using small molecule inhibitors of cyclin-dependent kinases to induce cell-cycle arrest at G1 phase [5-7], 3) anti-CTLA4 checkpoint inhibitors to enhance anti-tumor immune response $[8,9], 4)$ telomerase inhibitors to prevent telomere elongation $[10,11], 5)$ vascular endothelial growth factor (VEGF) inhibitors to inhibit angiogenesis [12-14], 6) PARP inhibitors to prevent repair of single strand breaks (that will converted into double strand breaks if they persist), and induce synthetic lethality if combined with mutations in genes involved in repair of double strand breaks such as BRCA [15], 7) c-MET inhibitors to prevent metastasis and invasion [16-18], 8) pro-apoptotic BH3 protein (member of the Bcl-2 family) mimetics $[19,20], 9)$ aerobic glycolysis inhibitors to prevent conversion of glucose into pyruvate in an oxygen-independent manner for energy production (especially useful for the tumor in oxygen deprived hypoxic areas) [21-23], or 10) antiinflammatory drugs to inhibit the pro-tumorigenic effects of inflammation [24, 25]. However, in practice, most patients do not show durable responses and eventually relapse. 


\section{Tumor Resistance}

Resistance to chemotherapy, radiotherapy, and personalized precision treatments is a major problem underlying treatment failure and limiting durable responses and cures. The mechanisms of resistance to these treatments include molecular alterations of the drug target, activation of pro-survival pathways, metabolic inactivation of drugs, and ineffective induction of cell death [26]. Moreover, molecular tumor analysis of patient genomes has shown high interpatient and intratumor genetic heterogeneity. This heterogeneity is already present prior to treatment or it develops during treatment, and it is thought to be one of the key contributing factors to clinical therapeutic resistance [27].

Retrospective analyses of phase II clinical trials indicate that a personalized treatment approach results in better outcomes and fewer treatment toxicity than conventional therapies [28]. Genetic profiling of patients also improves the selection of drugs best suited for clinical implementation. Currently, the likelihood of antineoplastic drugs to be approved in phase I clinical trials, compared to drugs for other diseases, is $\sim 6.7 \%$ ( $\mathrm{n}=1803$ ) [29]. The success rate of drugs that are approved throughout all clinical phases is of $19.4 \%$ [30], a parameter that is grossly underestimated due to false negative drugs that are a result of inadequate screening. An example of the latter is the case of the EGFR inhibitor Gefitinib which was initially approved by the FDA (Food and Drug Administration) in 2003 for advanced non-small cell lung cancer (NSCLC) patients previously treated with chemotherapy, where the response rate of refractory patients was $\sim 10 \%$ [31]. This consent was later on withdrawn in 2005 when Gefitinib didn't show significant improvement in the setting for which it was accepted [32]. Nonetheless, when the clinical trial outcome was assessed based on EGFR oncogenic mutation status, patients bearing the mutation had prolonged progression-free survival compared to standard first-line carboplatin-paclitaxel doublet treatment [33] and FDA approval was regained in 2009 in Europe. In this decade, an average of 35 drugs per year are discontinued in different phases of development, whereby those in the last phases are especially problematic due to the high expenses in money, patients and research resources. This failure rate could be improved if drug programs followed a biomarker-based strategy, if proof of concept was obtained, and if all negative results on clinical trials were published [34].

In this thesis, I have focused on enhancing treatment outcome in lung and brain cancer. 


\section{Lung Cancer}

Lung cancer can be classified into two main types: non-small cell lung cancer (NSCLC) and small cell lung cancer (SCLC) accounting for $85 \%$ and $15 \%$ of all lung cancer cases, respectively. NSCLC can be further subdivided into adenocarcinoma, squamous cell carcinoma, and large cell carcinoma. Staging is based on tumor location and infiltrative nature. NSCLC can remain localized in the lung (stage I), or infiltrate nearby lymph nodes (stage II), colonize the chest (stage III), and other body organs (stage IV). According to the American Cancer Society, the 5-year survival rate for stage I NSCLC patients is between $45-49 \%$, for stage II is $30 \%$, for stage IIIA is $14 \%$ and IIIB is $5 \%$, and finally, for stage IV is $1 \%$. In SCLC, there are only two stages: limited or extensive (when it has colonized the chest and other body organs). SCLC is usually more responsive to chemotherapy and radiotherapy than NSCLC but its fast growing, infiltrative/invasive and aggressive nature makes it challenging to obtain cures even after dose intensification [35]. Staging of lung cancer is usually determined by Positron Emission Tomography (PET) imaging but the golden standard to assess molecular alterations is tissue biopsy. Obtaining representative and reliable tissue biopsies in lung cancer for gene mutation profiling is challenging due to intratumor heterogeneity, risk of pneumothorax and bleeding and difficult anatomical accessibility of biopsy target. Because tumors may alter their mutation status throughout treatment (especially with tyrosine kinase inhibitors where it has been well documented, but probably also with other treatments), dynamic acquisition of tumor biopsies in time has been recommended [36].

This thesis focuses on NSCLC subtypes where current first-line treatment consists of surgical resection, chemotherapy, radiation, targeted therapy and combinations thereof. Selection of treatment is principally based on TNM (Tumor-NodeMetastases) stage, performance status and presence of oncogenic driver mutations. More than two-thirds of NSCLC patients are diagnosed when cancer is inoperable and locally advanced. At this stage, patients receive concurrent polychemotherapy with fractionated radiation (mostly delivered in 2Gy fractions for a total dose of 60Gy). Concurrent chemoradiation has proven to be better than the sequential combination in improving loco-regional control, overall and progression-free survival [37]. Chemotherapy often involves the use of two or more agents where one of them is usually platinum-based (cisplatin, carboplatin) combined with another one with a different mechanism of action. The use of concomitant polychemotherapy (double or triple) regimens as compared to single-agent chemotherapy has been shown to improve progression-free survival of patients with locally advanced 
NSCLC [37]. The late diagnosis and the intrinsic and/or acquired treatment resistances remain obstacles in improving cancer survival.

Low dose computed tomography (CT) screening of patients for lung cancer is considered for high risk subjects ( $>49$ years, current/former smokers), although it has rendered some controversy. Whereas Henschke et al., claim that annual spiral CT screening of asymptomatic populations at risk of lung cancer would increase 5-year survival rates from $16 \%$ by routine care to $86 \%$ in a cost-effective manner [38], Bach et al. report that this approach would lead to over diagnosis (false positives) and overtreatment of indolent cancers thus resulting in no additional clinical benefit [39]. It should be noted that $\mathrm{CT}$ screening identifies a greater proportion of patients bearing lung cancers with long doubling times ( $>400$ days versus 135 days by routine care). Consequently, both cancer populations (slow versus faster growing tumors) shouldn't be managed in the same way to avoid overtreatment / undertreatment [40]. Large-scale clinical implementation however, is not recommended yet because there are several parameters that remain to be optimized such as: precise definition of the high-risk population, timing and frequency, type of CT (2D versus $3 \mathrm{D}$ ), managing false positive cases, and cost-effectiveness as compared to smoking cessation [41]. Over $50 \%$ of lung adenocarcinomas in Europeans bear driver genetic alterations: $\sim 25-38 \%$ of them in KRAS, $\sim 7-16 \%$ in EGFR, $~ 5 \%$ in MET, $\sim 3-5 \%$ ALK, $\sim 2-5 \%$ in BRAF, and, $\sim 2 \%$ in RET [42-44]. Clinical application of diagnostic tests for KRAS genotyping, detection of actionable EGFR variants, and/or ALK translocations remains challenging [45-47]. These studies bring to light that lung cancer should be approached as a disease with a wide spectrum of behaviors and responses, and that there is a great need for personalizing precision treatment to improve survival.

\section{Glioblastoma Multiforme (GBM)}

Brain tumors comprise a diverse group of cancers that either arise from cells in the central nervous system, or from tumors that metastasized from lung, melanoma or breast. Meningiomas and glial tumors including glioblastoma, astrocytoma and oligodendroglioma, account for two-thirds of all brain tumors in adults. Glioblastoma is the most prevalent and aggressive primary brain tumor in adults, and it arises from supportive brain cells: the astrocytes. According to the Central Brain Tumor registry of the USA, 3.19 subjects per 100000 will develop glioblastoma, an incidence which increases with age. In the Netherlands, about 600 new patients are diagnosed with glioblastoma every year. There are two main types: primary or de 
novo which is the most common and aggressive form, or secondary which grows slower and arises from low-grade glioma. Standard of care includes tumor debulking by surgery and concurrent chemotherapy with fractionated radiotherapy (delivered in 2Gy-fractions to a total of 60Gy) whereby median survival doesn't exceed 15 months. Patients with a methylated MGMT (O (6)-methylguanine DNAmethyltransferase) promoter, involved in DNA repair, are sensitive to alkylating agents such as temozolomide, and have significantly better treatment outcomes [48]. Hence, temozolomide is part of standard of care for most GBM patients. MGMT methylation status is a promising biomarker to stratify GBM patients prior to treatment however, tumors can change their methylation status post-therapy [49], and tumor progression is inevitable. GBM remains an incurable disease.

A better understanding of GBM pathophysiology and mechanisms of recurrence would improve patient stratification [50]. There at least 5 clinical subtypes with distinct molecular alterations [51]. The two glioblastoma subtypes also have different genetic mutation profiles: while primary or "de novo" GBM have EGFR amplification, PTEN mutation, and absence of IDH (Isocitrate dehydrogenase) mutations; secondary GBM lack EGFR amplification but have TP53 and IDH mutations [51], and additionally, mutations in ATRX (Alpha thalassemia/mental retardation syndrome X-linked) [52]. GBM is a highly heterogeneous disease. One of the resistance-generating mechanisms in GBM is mediated by TP53 gain-of-function mutations which confers poor overall survival prognosis due to increase in MGMT expression [53]. Combination therapies using EGFR and VEGF inhibitors in recurrent GBM subjects, have reported slightly improved survival (cediranib plus gefitinib [54]) or no survival benefit (bevacizumab plus sorafenib [55]).

Despite efforts in personalizing GBM therapies, clinical responses to targeted therapies so far have been marginal, in particular for recurrent cases, possibly due to low blood brain barrier (BBB) permeability or inadequate patient screening [56]. There is hence, a dire need for novel disease-tailored precision treatments.

\section{Stem Cell pathways: Targets for Precision Therapy}

Emerging evidence implicates a subpopulation of tumor cells (cancer stem cells) with characteristics of normal stem cells such as unlimited self-renewal, capacity for multipotent differentiation, and high tumor initiating ability [57], that are resistant to treatment [58]. Both in lung and brain cancers, such cancer stem cells have been identified and associated with worse outcome [59, 60]. 
NOTCH signaling regulates cellular activities in different ways such as promotion or repression of cellular proliferation, controlling differentiation and cell death [61] as well as maintenance of the stem cell population [62].

This thesis describes translational research aimed at improving therapeutic efficacy via combination of therapies used as part of standard of care for NSCLC and GBM with NOTCH signaling inhibition.

\section{NOTCH: A Historical Perspective}

In the early $20^{\text {th }}$ century, Dexter described a mutant notched wing phenotype in Drosophila melanogaster [63]. Genetic studies undertaken by Thomas H. Morgan identified diverse X-linked Notch phenotypes, dominant with regards to wing morphology and bristles, but recessive in their lethality [64]. Poulson was the first to describe a recessive neurogenic phenotype of Notch [65], mediated by binding to the Notch ligand Delta [66], and its involvement in fly embryogenesis. In worms, it was first identified that Notch is a genetic switch for cell-fate decisions and its effect is mediated by interactions between cells [67].

In 1983, the locus of the notch gene was cloned and sequenced from flies to mammals. The gene identified encodes for a single pass transmembrane glycoprotein protein containing a large extracellular domain consisting of multiple repeats of the epidermal growth-factor (EGF)-like motif $[68,69]$. Notch receptors lacking the EGF-like repeats were found to be constitutively active in several organisms including Drosophila melanogaster [70, 71], Caenorhabditis elegans [72], and Xenopus [73]. Drosophila melanogaster's genome contains only one Notch receptor, whereas Caenorhabditis elegans' genome contains two that are biochemically but not functionally interchangeable (lin-12 and glp-1 [74]). Mammalian cells encode for four NOTCH receptors (NOTCH 1-4). The first identification of NOTCH1 as an oncogene was done by Ellisen et al. who reported that TAN-1, now called NOTCH1, chromosomal translocations occurred in T-cell acute lymphoblastic leukemia (TALL) patients and led to hyperactivated gene activation [75]. Shortly after, mammalian NOTCH2 was identified in rats and presented similar structural motifs than NOTCH1 but different spatial and temporal expression patterns [76]. The third NOTCH receptor was identified in mice (mNOTCH3), it lacked specific EGF-like repeats, and encoded a shorter intracellular domain as compared to the two other NOTCH receptors. mNOTCH3 is thought to have a pivotal role in the development of the central nervous system [77]. The fourth mammalian NOTCH homologue 
(NOTCH4) was found to have less EGF-like repeats than the rest of the NOTCH homologues, is thought to have a role in vertebrate endothelium, and has a role as a proto-oncogene in breast carcinogenesis [78].

Studies in D. melanogaster and $C$. elegans have shown that only the intracellular domain of the Notch receptor (Lin-12 in C. elegans) is implicated in signal transduction, resulting in regulation of neural/epidermal cell fate. The extracellular and transmembrane domains are involved in negatively regulating the intrinsic activity of the uncleaved receptor [72, 79]. The Notch receptor can only exert its function in development in the presence of a nuclear localization signal in its protein sequence. CBF1, now called CSL or RBP-Jk (Recombination signal binding protein $\mathrm{Jk}$ ), is a sequence specific DNA-binding protein that associates with the NOTCH intracellular domain. It wasn't until it was shown that CBF1 stimulates transcription of the downstream NOTCH target HES1 (through the KBF2-binding site of HES1) in the presence of the NOTCH $\triangle \mathrm{E}$ truncated form but not the full-length $\mathrm{NOTCH}$ receptor, that a cleavage model for $\mathrm{NOTCH}$ activation and subsequent gene transcription was accepted [80]. Notch proteins are cleaved at different sites, of which one, is performed by the $\gamma$-secretase multi-enzymatic protein complex which is also involved in the generation of beta-amyloid peptide plaques in brain [81], whose accumulation leads to Alzheimer's disease [82]. Sherrington et al. discovered in 1995 a new integral protein, the $\mathrm{S} 182$ transcript from the $A D 3$ gene (now called presenilin 1), which had missense mutations causative of an aggressive form of familial Alzheimer's disease of early onset [83]. Studies in C. elegans and D. melanogaster identified three other components of the $\gamma$-secretase besides presenilin 1: pen-2, aph-1, and nicastrin [84], that act together to process both $\beta$-APP and Notch receptors $[85,86]$. Notch receptor cleavage results in the release of the intracellular thus enabling signal transduction [86] and gene transcription.

\section{NOTCH Signaling}

Notch, WNT and Hedgehog $(\mathrm{HH})$ signaling pathways act, and interact together, to maintain tissue homeostasis, a regulated balance between the proliferation of undifferentiated cells and their differentiation into mature cell types. WNT/ $\beta$-catenin has been shown to directly bind the NICD, resulting in an increased transcriptional output of target genes [87]. MAML1, essential for NOTCH signaling activation, has been reported to function as a co-activator for $\beta$-catenin-dependent transcription [88]. A tight regulation of these pathways ensures normal physiological functions.

Nowadays, the canonical NOTCH signaling pathway is well-established and is identified as a highly conserved short-range signaling pathway that needs of two 
adjacent cells to communicate: one expressing a NOTCH receptor and the other expressing a ligand. Alongside the canonical pathway, a non-canonical Notch signaling pathway has been described in mammals and flies. The latter is $\gamma$-secretasedependent but RBP-JK-independent, although its mechanism remains unclear. Noncanonical ligands, such as DLK1 (Delta-like 1 homolog), may interact with themselves through specific EGF repeats [89] or with extracellular matrix proteins to unfold the negative regulatory region (NRR) and activate Notch. This pathway is also thought to exert its function by post-translationally antagonizing Wnt/ $\beta$-catenin [90].

NOTCH receptors are large type I transmembrane glycoproteins that have three main structural domains: the extracellular (ECD), the transmembrane (TMD), and the intracellular domains (ICD). The extracellular domain in N-terminal consists of 2936 epidermal growth factor (EGF)-like tandem repeats which mediates interactions with the NOTCH ligands, many of which occur via calcium binding [91]. The EGFlike repeats can be post-translationally modified via O-glycosylation and/or Ofucosylation to regulate NOTCH signaling [92]. The EGF repeats are followed by a negative regulatory region (NRR), composed of a Lin-12-NOTCH repeats (LNR) and a heterodimerization domain (HD), which prevents pathway activation in the absence of ligand. The NRR is followed by a transmembrane domain (TMD) which contains a "stop translocation" signal of Arg/Lys residues. Subsequently, the RAM

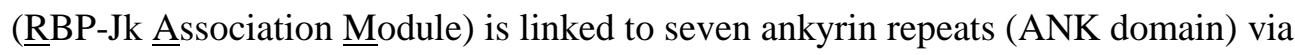
a linker containing the first part of a bipartite nuclear localization signal (NLS). The evolutionary divergent transactivation domain (TAD) is followed by the second part of the NLS. And finally, in the C-terminal end, there is a proline/glutamic acid/serine/threonine-rich motif (PEST) in charge of regulating the stability of the cleaved NOTCH intracellular domain (NICD) [93]. NOTCH ligands that are type I transmembrane proteins, are composed of three structural domains: N-terminal DSL (Delta/Serrate/LAG-2) domain, the DOS (Delta and OSM-11-like proteins) domain containing EGF repeats, and another subset of EGF repeats (both calcium binding and non-calcium binding) [94, 95]. In mammals, DSL ligands can be classified in two groups: Delta-like (DLL1, DLL3, and DLL4) and Jagged-like (JAGGED1 and JAGGED2) [93]. The DOS domain, found in the first two EGF-like repeats, is known to cooperate with the DSL domain to mediate NOTCH binding and signaling [94]. Mutations in the DOS motif of Jagged1 may cause Alagille syndrome [96] or Tetralogy of Fallot [97]. 

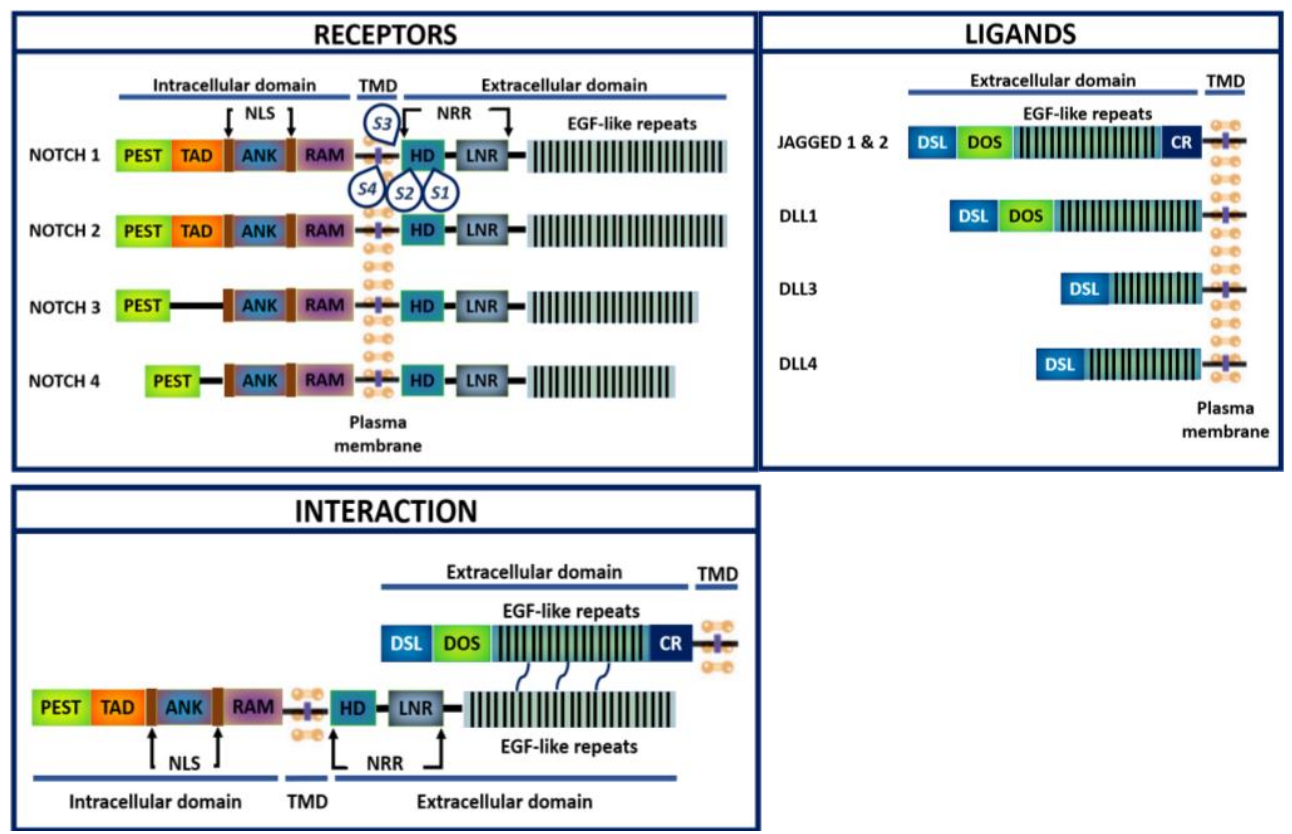

Figure 1: NOTCH receptors and ligands from the canonical NOTCH signaling pathway and their interaction. See text for details.

NOTCH receptor maturation starts in the secretory pathway after cleavage by furinlike proteases which generate a non-covalently linked heterodimer, the NECDNTMIC (Notch extracellular domain-Notch transmembrane and intracellular domain). Furin-like proteases cleave at Site 1 (S1), which is located within the HD of the NRR. Once this NOTCH receptor-ligand interaction takes place, leading to the formation of homodimers between EGF repeats, the NOTCH receptor undergoes two consecutive proteolytic cleavages that will render the receptor active. Ligand binding unfolds the NRR, opening up a scissile bond for the ADAM10 metalloproteinases $[98,99]$ at Site 2 (S2), 12 amino acids upstream of the TMD and in the inner core of the NRR, causing a cleavage of the NOTCH ectodomain, thus leaving a membrane-tethered Notch-extracellular truncation (NEXT). NEXT is the substrate for $\gamma$-secretase, a multi-component protease complex, which cleaves at Site 3 (S3), in the inner part of the TMD, and is further cleaved until Site 4 (S4), in the middle of the TMD. The resulting Notch intracellular domain (NICD) translocates to the nucleus and binds to the DNA-bound protein CSL (RBP-Jk) via the RAM domain and replaces the corepressors (CoR). The ANK domain also binds to the CSL to recruit the coactivator (CoA) Mastermind/Lag-3 and the mediator MED8, to activate 
gene transcription [100]. Some of the NOTCH downstream targets include: HES and HEY protein families, and oncogenes like RAS or MYC [101].

NOTCH signaling is spatially, and temporally, tightly controlled. The PEST domain of the NICD is phosphorylated by the CDK8 kinase, and thus targeted for proteasomal degradation by E3 ubiquitin ligase Sel10/Fbw7/cdc4 to target NICD for degradation $[102,103]$.

\section{NOTCH Endocytosis}

Another mechanism by which NOTCH signaling is controlled is via NOTCH receptor and ligand internalization by endocytosis resulting in either positive or negative feed-back loops in a context-dependent manner. Unstimulated / inappropriately stimulated NOTCH receptors are recycled or degraded mediated by ubiquitination-dependent endocytosis. Endocytosis of DSL-ligands, triggered by monoubiquitinylation by E3 ligases (Neuralized for Delta-type or Mindbomb for Serrate/Jagged-type ligands), plays an important role in upregulating signaling activity [104]. E3 ubiquitin ligases (e.g. NUMB, Deltex, Nedd4, Cbl) also controls NOTCH receptor trafficking towards lysosomal degradation or recycling through endocytosis [105]. Numb interacts with the cytosolic HECT (Homologous to E6-AP C Terminus) domain-containing E3 ligase Itch to promote ubiquitination and degradation of membrane-tethered NOTCH1 [106]. Numb interacts with proteins involved in endocytic trafficking, including Eps15, the EHD/Rme-1 family of proteins, and the clathrin adaptor protein, AP2 [107], to modulate NOTCH1 receptor signaling. Components of ESCRT (endosomal sorting complex required for transport) complexes, which regulate sorting into multivesicular bodies, may function as tumor suppressors by preventing Notch activation [108]. The composition of the phospholipid membrane can also affect both NOTCH and EGFR signaling in Drosophila. The rate-limiting enzyme for phosphatidylcholine biosynthesis, phosphocholine cytidylyltransferase 1, regulates endocytosis and in its absence, NOTCH and EGFR accumulate in enlarged endosomes, which is correlated with a decrease in signaling strength, thus suggesting that accumulation in endosomal compartments is for lysosomal degradation or for spatial separation from activating molecules (e.g. $\gamma$-secretase or ligands) [109]. On the other hand, protein sorting in lipid rafts, involved in receptor endocytosis, is a way to positively regulate Notch receptor activation in C. elegans [110]. Notch receptor endocytosis is also regulated by physical stimuli. In order to ensure thermal regulation during Drosophila's development, Notch is internalized via Deltex-mediated endocytosis, 
allowing signaling activation at low temperatures, whereas at high temperatures, Suppressor of Deltex $(\mathrm{Su}(\mathrm{dx}))$ mediates Notch endocytosis in a cholesteroldependent manner, leading to Notch degradation [111].

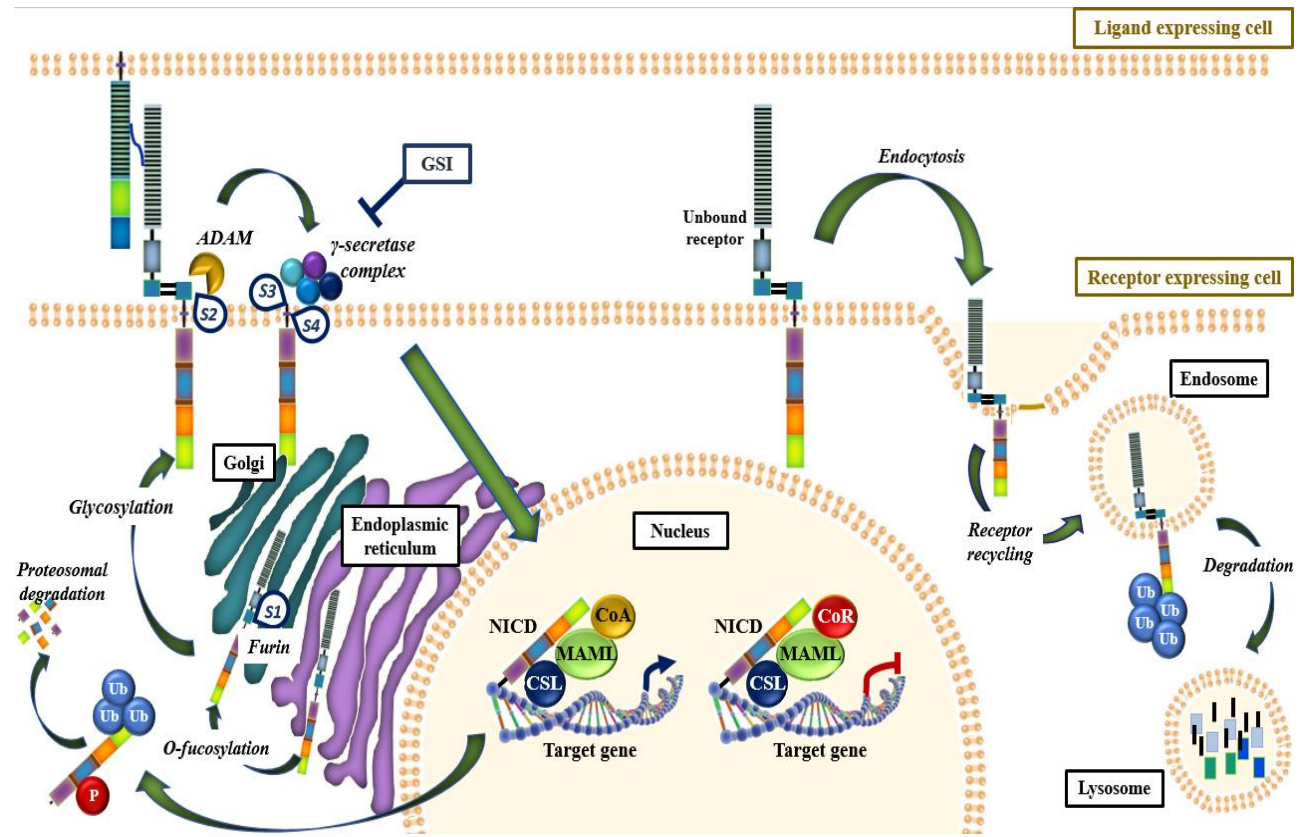

Figure 2: Overview of the canonical NOTCH signaling pathway (modified from Life Sciences). See text for details. ${ }^{*} \mathrm{GSI}=\gamma$-secretase inhibitor

\section{NOTCH in Normal Physiology}

Notch signaling has essential roles during development, cell proliferation or death, determination of cell-fate, differentiation, and maintenance of stem cells in different organs [105, 112]. NOTCH signaling is essential for normal lung organogenesis where it regulates broncho-alveolar and neuroendocrine cell differentiation [113]. Additionally, NOTCH activation promotes differentiation of basal cells into secretory lineages whereas its inhibition leads to differentiation into ciliated cells [114].

In the brain, NOTCH signaling regulates cellular differentiation via lateral inhibition, an important mechanism controlling neural cell fate during development [115]. Notch signaling maintains neural stem and progenitor cells by decreasing expression of Hes1, Hes5, and proneural genes, it regulates glial differentiation, and blocks 
neural differentiation via HES1 [115]. In normal cerebellar development, NOTCH2 is predominantly expressed in proliferating progenitors and NOTCH1 in post-mitotic differentiating cells [116].

Expression analysis and lineage tracing portrayed redundant roles of NOTCH1 and 2 in intestinal homeostasis [117-119]. Blockade of NOTCH ligands Dll1/4 results in goblet metaplasia and proliferative arrest of crypt epithelial cells [120, 121]. Multipotent crypt progenitors are maintained by active NOTCH and WNT pathways, and their inactivation results in differentiation into secretory [117, 119] or enterocytic $[122,123]$ fates, respectively. Several Notch signaling pathway members are implicated in vasculature: in the endothelium (NOTCH1/4; DLL1/4; HES1; and HEY1/2) and in surrounding mural cells (NOTCH3) [124]. DLL4 is positively regulated by several pro-angiogenic factors including: growth factors (VEGF-A, bFGF) $[125,126]$; interleukin 6 mediated by STAT3 activation in stromal cells [127]; Foxc proteins via direct activation leading to arterial specification and lymphatic sprouting [128]; N4 ICD [129]; and HIF-1 $\alpha$-mediated upregulation of DLL4-NOTCH-HEY2 consequently repressing COUP-TFII, a vein identity regulator, in endothelial progenitor cells to specify cell fate [130]. NOTCH also participates in many other functions including keratinocyte differentiation and growth arrest [131], T cell maturation [132, 133], and is a key regulator of cell fate decisions in the hematopoietic system regulating megakaryocyte development, and the myeloid and erythroid lineages [134].

\section{NOTCH in Lung and Brain Cancers}

NOTCH signaling is deregulated in multiple human diseases including developmental syndromes (e.g. Aortic valve disease, Alagille) $[112,135]$ and adultonset diseases (e.g. Alzheimer, hematological and solid cancers) [136, 137]. Both lung cancer and glioblastoma are characterized by aberrant NOTCH signaling. In NSCLC, activating mutations in NOTCHI or loss of the negative regulator of $\mathrm{NOTCH}, N U M B 1$, are present in $10 \%$ and $30 \%$ of adenocarcinomas [138]. High NOTCH activity in NSCLC is associated with worse disease-free survival in patients [139], and increased proliferation, greater hypoxic fraction, and radiation resistance in NSCLC tumor-bearing mice [140]. In contrast, in squamous NSCLC, NOTCH mutations are usually inactivating and function as tumor suppressors [141].

In glioblastoma, high Deltex1, a positive regulator of NOTCH signaling [142], expression induces PI3K and MAPK/ERK pro-mitotic pathways, anti-apoptotic Mcl- 
1, and promotes migration/invasion in a NOTCH, miR-21, and ERK-mediated manner, and is associated with poor prognosis in glioblastoma [143].

NOTCH1 regulates transcription of EGFR, frequently overexpressed/amplified in GBM, via TP53 [144], and in this subpopulation of GBM cells with overexpressed EGFR, Notch signaling genes are concurrently significantly overexpressed [145]. Both NOTCH1 and 2 have been implicated in radiation resistance of GBM stem cells by upregulating AKT and downregulating the pro-apoptotic protein MCL-1 [146]. NOTCH inhibition reduces glioma subpopulations expressing CD133, NESTIN, SOX2, BMI1, and OLIG2 stem cell markers implicated in treatment resistance, and attenuates tumoral neurosphere and xenograft growth, mainly promoted by NOTCH2 [147, 148].

Overall, these data implicate NOTCH signaling in lung and brain cancer development and treatment resistance.

\section{Duality of NOTCH activity in Cancer}

Aberrant NOTCH signaling in cancer patients can act as an oncogene or tumor suppressor gene. Mechanisms that result in aberrant NOTCH signaling include receptor/ligand overexpression, activating / inactivating mutations, epigenetic regulation, and post-translational modifications. The outcome of aberrant NOTCH activity is highly context- and cancer stage-dependent, and is defined by interactions with the microenvironment. Moreover, NOTCH activity is influenced by means of pathway-crosstalk including those pathways involved in epithelial-mesenchymal transition, neurogenesis, hematopoiesis, angiogenesis, and cancer stem cell maintenance.

There are differential mutational patterns of NOTCH pathway members in cancers that lead to divergent roles in tumorigenesis (reviewed in [149]):

1) Autonomous oncoproteins: Mutations that disrupt the NRR in NOTCH1 together with loss of the PEST domain are frequent in T-ALL, triple-negative breast cancer, adenoid cystic carcinoma, and in tumors derived from pericytes or smooth muscle. These mutations result in ligand independent NOTCH receptor proteolysis and activation.

2) Microenvironment-dependent oncoproteins: Mutations that affect only the PEST domain are prevalent in B-cell tumors (chronic lymphocytic leukemia, splenic marginal zone lymphoma, mantle cell lymphoma), peripheral T-cell lymphomas, and breast cancers [150]. These mutations lack the selective pressure for ligandindependent $\mathrm{NOTCH}$ activation, and activation is probably restricted to selective microenvironments where tumor cells interact with stromal ligands. 
3) Autonomous tumor suppressors: Mutations in the N-terminal region of the NOTCH receptor are predominant in squamous cell carcinomas (of the skin, head and neck, esophagus, and lung) and in small cell lung cancer, urothelial carcinoma, and low-grade glioma. These mutations lead to loss of protein functionality (failure to produce the NOTCH protein or formation of dominant negative decoy receptors lacking ICD).

4) The consequences of the loss or downregulated RBP-Jk copy number is dependent on tumor type. In tumors where NOTCH functions as an oncogene (e.g. breast cancer) NOTCH downstream targets will be upregulated, probably due to the loss of corepressor recruitment by RBP-Jk. However, in cancers where $\mathrm{NOTCH}$ acts as a tumor suppressor (e.g. squamous cell carcinoma) NOTCH downstream targets will be inhibited.

\section{NOTCH as an Oncogene}

The first report of a pathological and NOTCH-dependent carcinogenic event was described in an aggressive form of T-cell acute lymphoblastic leukemia (T-ALL), which affects white blood cells, occurring mainly during childhood. Notch1 chromosomal translocations ("TAN-1"), a rare mutation in T-ALL patients, result in hyperactivated NOTCH1 gene expression leading to T-cell leukemia [75]. While these translocations are rare, NOTCH1 mutations, resulting in constitutively active NOTCH signaling, have been reported in $\sim 60 \%$ of T-ALL patients, and occur in the extracellular NRR or PEST domains [151]. Moreover, mutations in E3 ubiquitin ligase Sel10/Fbw7/Cdc4, that targets the NOTCH PEST domain for degradation, are frequently found in T-cell leukemias [152]. Fbw7 is a F-box family protein part of the Skp1, Cul1, and F-box protein (SCF) ubiquitin ligase complex. Fbw7 acts as a tumor suppressor in many tissues by targeting NOTCH, c-MYC, Cyclin E, c-JUN, and MCL-1 oncogenes for ubiquitin-mediated degradation [153,154]. The first study showing the oncogenic capacity of NOTCH proteins identified NOTCH4 as a retroviral insertion (int-3) in mammary tumors induced by mouse mammary tumor virus (MMTV) $[155,156]$. Following this, several mutations which enhance NOTCH signaling via either gain of function mutations in NOTCH receptors, or inactivation of negative regulators (e.g. NUMB, FBXW7), have been shown to contribute to tumor progression in many solid tumors: mutations in PEST, HD or extracellular domains of NOTCH1, 2, and/or 3, common hot spots in T-ALL, in triple negative breast cancer [150], upregulation of NOTCH1 or RBP-Jk in colon cancer [157], Fbw7 inactivation in pancreatic ductal adenocarcinoma [158, 159], gain-of function 
mutations in NOTCH1 or loss of NUMB expression in NSCLC [138], NOTCH1 upregulation in head and neck carcinoma [160], NOTCH1 activation or NUMB downregulation in prostate cancer [161, 162], and NOTCH1, 2, and 4 activation in anaplastic astrocytoma [163]. It has been shown that NOTCH drives tumorigenesis mainly by cell cycle progression and inhibiting apoptosis. NOTCH1 and 3 induce cyclin D1 expression, mediated by JAGGED1 binding, to promote proliferation $[164,165]$. NOTCH is frequently expressed in astrocytic gliomas and interacts with the EGFR pathway to regulate important drivers of glioma such as PI3K-AKT pathway, KRAS, cyclin D1 and matrix metalloproteinase 9 (MMP9) [166]. Additionally, there is mounting evidence supporting the role of NOTCH signaling in maintenance and self-renewal of resistant-cancer stem cells in cancers including TALL [167], brain [148, 168], breast [169], colon [170], and non-small cell lung cancer [139]. In these cases, NOTCH activation is associated with worse prognosis in survival.

\section{NOTCH as Tumor Suppressor}

There is an increasing body of literature demonstrating a tumor suppressive role for $\mathrm{NOTCH}$ proteins. In the epidermis, NOTCH suppresses tumor formation by blocking WNT/ $\beta$-catenin signaling to drive epidermal differentiation. Notchl deletion in epidermis results in the formation of basal cell tumors [171]. Loss of Notchl in epidermal keratinocytes impairs skin barrier integrity by creating a woundlike state that promotes tumorigenesis however, expression of NOTCH1 was insufficient to suppress this tumor-promoting effect, emphasizing the importance of crosstalk with stroma [172]. NOTCHI expression is under the direct transcriptional control of p53 in human keratinocyte tumors and can induce apoptosis by enhancing TP53 activity [171]. In HPV infected keratinocytes, the E6 oncoprotein binds to and mediates p53 degradation, and the viral protein NFX1-123 activates NOTCH signaling to increase keratinocyte differentiation in the absence of cell cycle arrest [173]. In human squamous cell cancer (SCC), NOTCH also functions as a tumor suppressor including head and neck SCC [174], cutaneous SCC [175], lung SCC [141], bladder SCC [176], and esophageal SCC [177]. Genetic alterations in SCC are mostly missense mutations, nonsense mutations resulting in truncated proteins lacking the TAD domain, or frame shift mutations in NOTCH1, and less frequently, in NOTCH2 and NOTCH3. 


\section{NOTCH-based Therapeutic Interventions}

There are over 100 preclinical studies and 37 registered interventional clinical trials targeting the NOTCH pathway (www.clinicaltrials.gov). Most of these studies use small molecule $\gamma$-secretase inhibitors (GSI) that block the rate-limiting step in NOTCH activation: the intramembranous cleavage by presenilin containing the $\gamma$ secretase enzyme. There are several GSI in clinical trials including: BMS-906024, PF-3084014, RO4929079, MK-0752. Additionally, there are monoclonal antibodies against the NRR (to keep receptors in a proteolysis resistant conformation), the ligand-binding EGF repeats in the ECD, or against NOTCH ligands (e.g. DLL4 in endothelial cells, to target tumor angiogenesis). Alternative methods of NOTCH signaling inhibition that have not proceeded to clinical trials include cell-permeable, synthetic, blocking peptides that interfere with the formation of NOTCH-CSLMAML activation complex, and natural non-toxic compounds, present in fruits, vegetables or nuts (e.g. delta-tocotrienol in blueberries; isoflavone genistein in soy products; resveratrol in grapes, red wine, and peanuts; and curcumin). Strong evidence regarding the NOTCH inhibitory property of natural compounds is lacking.

The dose limiting toxicity of NOTCH inhibitors is in the gastrointestinal tract where it causes goblet cell metaplasia resulting in diarrhea and dehydration. There are ways to overcome such toxicity by applying discontinuous scheduling, administering lower doses, and combination of NOTCH inhibitors with glucocorticoid treatment [178-180].

In contrast, when NOTCH acts as a tumor suppressor, therapeutic strategies will aim at promoting $\mathrm{NOTCH}$ activity to eliminate stem/progenitor cells by inducing their differentiation [181]. However, there are few studies addressing this approach.

Further discussion of the implications of oncogenic NOTCH signaling in nonsquamous NSCLC in survival, resistance to treatment, and therapeutic options for its targeting, will be discussed throughout this thesis. 


\section{Outline}

In this thesis, we have investigated the potential of NOTCH signaling as a therapeutic target in non-squamous NSCLC and GBM. We developed novel in vitro and in vivo models to investigate whether NOTCH inhibition using small molecule inhibitors (GSI), when used in combination with standard of care conventional treatment (chemotherapy and radiotherapy), improves treatment response.

Chapter 2 reviews the intrinsic and acquired treatment-based resistances that have arisen in NSCLC which are directly or indirectly associated with Notch signaling. We discuss how the NOTCH signaling pathway is associated with intrinsic mechanisms of tumor resistance including its role in: cancer stem cells, ABC drug transporters, epithelial-mesenchymal transition, hypoxia, and crosstalk with other oncogenic pathways such as with TP53, growth factor signaling (e.g. EGFR), tumor angiogenesis (e.g. VEGF), and the DNA damage response (ATM). We describe that NOTCH-based therapeutic regimens in combination with radiation, and chemotherapeutics/targeted agents is a promising strategy to combat NSCLC.

In Chapter 3, we evaluated in vitro in 2D, and in 3D multicellular tumor spheroids, whether addition of NOTCH inhibition to chemo- and chemoradiation regimens could delay significantly and synergistically tumor cell growth.

In Chapter 4, we tested in ectopically subcutaneously growing NSCLC xenografts, single NOTCH inhibition, cisplatin, and single dose irradiation regimens to determine the doses that rendered efficacy but no normal tissue toxicity. In a followup conditional study, the selected single doses of GSI and cisplatin, or of GSI and $\mathrm{RT}$, were combined to assess whether NOTCH inhibition could overcome treatment resistance in two models of NSCLC with different NOTCH expression levels.

In Chapter 5, we develop an orthotopic NSCLC preclinical model in mice where H1299 tumors grow as a single localized nodule and can be monitored using two positively correlated imaging modalities: dual wavelength BLI and dual energy CBCT. Additionally, we comment on different tumor volume-based radiation treatment plan set-ups that can be delivered employing an image-guided, high precision small animal irradiator taking into account tumor margins, and radiation dose-volume histogram parameters to both the internal and planed target volume and organs at risk (lung, heart, spinal cord). 
In Chapter 6, we investigated the efficacy of the triple combination therapy consisting on standard of care for glioma (temozolomide plus radiation) with a clinically approved NOTCH/gamma-secretase inhibitor. We reported that addition of NOTCH inhibition to standard of care delays 3D growth of glioma spheroids, reduces treatment resistant glioma stem cell markers in vitro and in vivo, and prolongs survival in mice with orthotopic glioblastoma.

Finally, Chapter 7 summarizes the findings of this thesis and comments on the future challenges and opportunities to be addressed in future research using NOTCH-based therapies.

\section{NOTCHing down Non-small cell lung cancer}

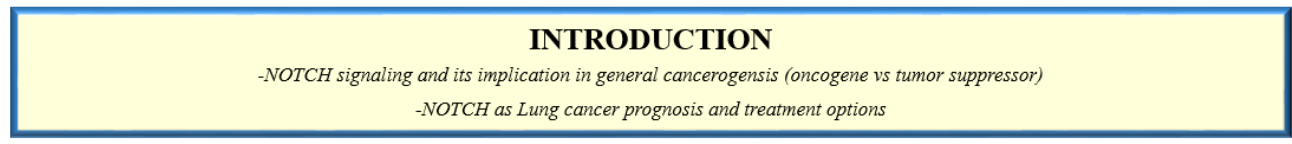

Chapter 2

NOTCH-associated treatment resistances

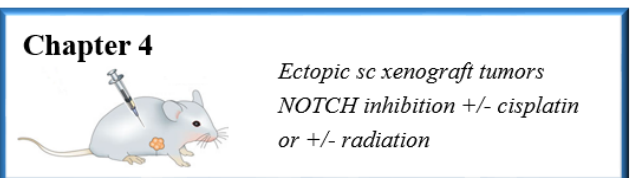

\section{Chapter 3}

$2 \mathrm{D}$ and $3 \mathrm{D}$ screens: $\mathrm{NOTCH}$

inhibition + /- Chemoradiation

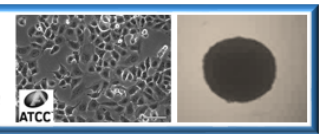

Chapter 5

Orthotopic xenograft tumors

Dual WL BLI and Dual energy CBCT

\author{
Chapter 6 \\ Orthotopic glioma model \\ $T M Z+/-R T+/-N O T C H$ inhibition
}

DISCUSSION AND FUTURE PERSPECTIVES

Figure 3: Schematic diagram of the outline of this thesis. 


\section{References}

1. Hanahan, D. and R.A. Weinberg, The hallmarks of cancer. Cell, 2000. 100(1): p. 57-70.

2. Hanahan, D. and R.A. Weinberg, Hallmarks of cancer: the next generation. Cell, 2011. 144(5): p. 646-74.

3. Han, W. and Y. Du, Recent Development of the Second and Third Generation Irreversible Epidermal Growth Factor Receptor Inhibitors. Chem Biodivers, 2017. 14(7).

4. Xu, M.J., D.E. Johnson, and J.R. Grandis, EGFR-targeted therapies in the post-genomic era. Cancer Metastasis Rev, 2017. 36(3): p. 463-473.

5. Hussain, A., C.K. Verma, and U. Chouhan, Identification of novel inhibitors against Cyclin Dependent Kinase 9/Cyclin T1 complex as: Anti cancer agent. Saudi J Biol Sci, 2017. 24(6): p. 1229-1242.

6. Polk, A., et al., Specific CDK4/6 inhibition in breast cancer: a systematic review of current clinical evidence. ESMO Open, 2016. 1(6): p. e000093.

7. Sobhani, N., et al., Cyclin dependent kinase 4 and 6 inhibitors as novel therapeutic agents for targeted treatment of malignant mesothelioma. Genes Cancer, 2017. 8(3-4): p. 495-496.

8. Hoos, A., Development of immuno-oncology drugs - from CTLA4 to PD1 to the next generations. Nat Rev Drug Discov, 2016. 15(4): p. 235-47.

9. Diesendruck, Y. and I. Benhar, Novel immune check point inhibiting antibodies in cancer therapy-Opportunities and challenges. Drug Resist Updat, 2017. 30(Supplement C): p. 39-47.

10. Bajaj, S., P.P. Roy, and J. Singh, 1,3,4-Oxadiazoles as telomerase inhibitor: Potential anticancer agents. Anticancer Agents Med Chem, 2017.

11. Wang, S., et al., BRD4 inhibitors block telomere elongation. Nucleic Acids Res, 2017. 45(14): p. 8403-8410.

12. Subbiah, V., et al., First-in-human trial of multikinase VEGF inhibitor regorafenib and anti-EGFR antibody cetuximab in advanced cancer patients. JCI Insight, 2017. 2(8).

13. Wilmes, L.J., et al., AG-013736, a novel inhibitor of VEGF receptor tyrosine kinases, inhibits breast cancer growth and decreases vascular permeability as detected by dynamic contrast-enhanced magnetic resonance imaging. Magn Reson Imaging, 2007. 25(3): p. 319-27.

14. Zhang, J., et al., The novel VEGF receptor 2 inhibitor YLL545 inhibits angiogenesis and growth in breast cancer. Oncotarget, 2016. 7(27): p. 41067-41080.

15. Farmer, H., et al., Targeting the DNA repair defect in BRCA mutant cells as a therapeutic strategy. Nature, 2005. 434(7035): p. 917-921.

16. Lev, A., et al., Preclinical rationale for combination of crizotinib with mitomycin $C$ for the treatment of advanced colorectal cancer. Cancer Biol Ther, 2017. 18(9): p. 694-704. 
17. Bouattour, M., et al., Recent Developments of c-Met as a Therapeutic Target in Hepatocellular Carcinoma. Hepatology, 2017.

18. Gozdzik-Spychalska, J., et al., C-MET inhibitors in the treatment of lung cancer. Curr Treat Options Oncol, 2014. 15(4): p. 670-82.

19. Wolf, P., BH3 Mimetics for the Treatment of Prostate Cancer. Front Pharmacol, 2017. 8: p. 557.

20. Karpel-Massler, G., et al., Targeting intrinsic apoptosis and other forms of cell death by BH3-mimetics in glioblastoma. Expert Opin Drug Discov, 2017. 12(10): p. 1031-1040.

21. Shan, S., et al., Apigenin Restrains Colon Cancer Cell Proliferation via Targeted Blocking of Pyruvate Kinase M2-Dependent Glycolysis. J Agric Food Chem, 2017. 65(37): p. 8136-8144.

22. Xie, R., et al., The Combination of Glycolytic Inhibitor 2-Deoxyglucose and Microbubbles Increases the Effect of 5-Aminolevulinic Acid-Sonodynamic Therapy in Liver Cancer Cells. Ultrasound Med Biol, 2017. 43(11): p. 26402650.

23. Ganapathy-Kanniappan, S. and J.F. Geschwind, Tumor glycolysis as a target for cancer therapy: progress and prospects. Mol Cancer, 2013. 12: p. 152.

24. Guadagni, F., et al., Non-steroidal anti-inflammatory drugs in cancer prevention and therapy. Anticancer Res, 2007. 27(5A): p. 3147-62.

25. Rayburn, E.R., S.J. Ezell, and R. Zhang, Anti-Inflammatory Agents for Cancer Therapy. Mol Cell Pharmacol, 2009. 1(1): p. 29-43.

26. Holohan, C., et al., Cancer drug resistance: an evolving paradigm. Nat Rev Cancer, 2013. 13(10): p. 714-26.

27. Bedard, P.L., et al., Tumour heterogeneity in the clinic. Nature, 2013. 501(7467): p. 355-64.

28. Schwaederle, M., et al., Impact of Precision Medicine in Diverse Cancers: A Meta-Analysis of Phase II Clinical Trials. J Clin Oncol, 2015. 33(32): p. 3817-25.

29. Hay, M., et al., Clinical development success rates for investigational drugs. Nat Biotechnol, 2014. 32(1): p. 40-51.

30. DiMasi, J.A., et al., Trends in risks associated with new drug development: success rates for investigational drugs. Clin Pharmacol Ther, 2010. 87(3): p. 272-7.

31. Dancey, J.E. and B. Freidlin, Targeting epidermal growth factor receptorare we missing the mark? The Lancet, 2003. 362(9377): p. 62-64.

32. Thatcher, N., et al., Gefitinib plus best supportive care in previously treated patients with refractory advanced non-small-cell lung cancer: results from a randomised, placebo-controlled, multicentre study (Iressa Survival Evaluation in Lung Cancer). Lancet, 2005. 366(9496): p. 1527-37.

33. Mok, T.S., et al., Gefitinib or carboplatin-paclitaxel in pulmonary adenocarcinoma. N Engl J Med, 2009. 361(10): p. 947-57. 
34. Jardim, D.L., et al., Factors associated with failure of oncology drugs in late-stage clinical development: A systematic review. Cancer Treat Rev, 2017. 52(Supplement C): p. 12-21.

35. Crivellari, G., et al., Increasing chemotherapy in small-cell lung cancer: from dose intensity and density to megadoses. Oncologist, 2007. 12(1): p. 79-89.

36. Jekunen, A.P., Role of rebiopsy in relapsed non-small cell lung cancer for directing oncology treatments. J Oncol, 2015. 2015: p. 809835.

37. Auperin, A., et al., Meta-analysis of concomitant versus sequential radiochemotherapy in locally advanced non-small-cell lung cancer. J Clin Oncol, 2010. 28(13): p. 2181-90.

38. International Early Lung Cancer Action Program, I., et al., Survival of patients with stage I lung cancer detected on CT screening. N Engl J Med, 2006. 355(17): p. 1763-71.

39. Bach, P.B., et al., Computed tomography screening and lung cancer outcomes. JAMA, 2007. 297(9): p. 953-61.

40. Detterbeck, F., The fruits of our efforts: time for a different view of lung cancer and CT screening. Thorax, 2009. 64(6): p. 465-6.

41. Vansteenkiste, J., et al., Early and locally advanced non-small-cell lung cancer (NSCLC): ESMO Clinical Practice Guidelines for diagnosis, treatment and follow-up. Ann Oncol, 2013. 24 Suppl 6: p. vi89-98.

42. Lin, J.J. and A.T. Shaw, Resisting Resistance: Targeted Therapies in Lung Cancer. Trends Cancer, 2016. 2(7): p. 350-364.

43. Schmid, K., et al., EGFR/KRAS/BRAF mutations in primary lung adenocarcinomas and corresponding locoregional lymph node metastases. Clin Cancer Res, 2009. 15(14): p. 4554-60.

44. Kerr, K.M., et al., Second ESMO consensus conference on lung cancer: pathology and molecular biomarkers for non-small-cell lung cancer. Ann Oncol, 2014. 25(9): p. 1681-90.

45. Vendrell, J.A., et al., Circulating Cell Free Tumor DNA Detection as a Routine Tool forLung Cancer Patient Management. Int J Mol Sci, 2017. 18(2).

46. Lin, C.C., et al., Emerging platforms using liquid biopsy to detect EGFR mutations in lung cancer. Expert Rev Mol Diagn, 2015. 15(11): p. 1427-40.

47. van der Wekken, A.J., et al., Dichotomous ALK-IHC Is a Better Predictor for ALK Inhibition Outcome than Traditional ALK-FISH in Advanced NonSmall Cell Lung Cancer. Clin Cancer Res, 2017. 23(15): p. 4251-4258.

48. Weller, M., et al., MGMT Promoter Methylation Is a Strong Prognostic Biomarker for Benefit from Dose-Intensified Temozolomide Rechallenge in Progressive Glioblastoma: The DIRECTOR Trial. Clin Cancer Res, 2015. 21(9): p. 2057-64.

49. Brandes, A.A., et al., O(6)-methylguanine DNA-methyltransferase methylation status can change between first surgery for newly diagnosed 
glioblastoma and second surgery for recurrence: clinical implications. Neuro Oncol, 2010. 12(3): p. 283-8.

50. Olar, A. and K.D. Aldape, Using the molecular classification of glioblastoma to inform personalized treatment. J Pathol, 2014. 232(2): p. 165-77.

51. Parsons, D.W., et al., An integrated genomic analysis of human glioblastoma multiforme. Science, 2008. 321(5897): p. 1807-12.

52. Jiao, Y., et al., Frequent ATRX, CIC, FUBP1 and IDH1 mutations refine the classification of malignant gliomas. Oncotarget, 2012. 3(7): p. 709-22.

53. Wang, X., et al., Gain of function of mutant TP53 in glioblastoma: prognosis and response to temozolomide. Ann Surg Oncol, 2014. 21(4): p. 1337-44.

54. Brown, N., et al., Multi-Center Randomized Phase II Study Comparing Cediranib plus Gefitinib with Cediranib plus Placebo in Subjects with Recurrent/Progressive Glioblastoma. PLoS One, 2016. 11(5): p. e0156369.

55. Galanis, E., et al., Phase II study of bevacizumab in combination with sorafenib in recurrent glioblastoma (N0776): a north central cancer treatment group trial. Clin Cancer Res, 2013. 19(17): p. 4816-23.

56. Lau, D., S.T. Magill, and M.K. Aghi, Molecularly targeted therapies for recurrent glioblastoma: current and future targets. Neurosurg Focus, 2014. 37(6): p. E15.

57. Reya, T., et al., Stem cells, cancer, and cancer stem cells. Nature, 2001. 414(6859): p. 105-111.

58. Baumann, M., M. Krause, and R. Hill, Exploring the role of cancer stem cells in radioresistance. Nature reviews. Cancer, 2008. 8(7): p. 545-554.

59. Bertolini, G., et al., Highly tumorigenic lung cancer CD133+ cells display stem-like features and are spared by cisplatin treatment. Proc Natl Acad Sci U S A, 2009. 106(38): p. 16281-6.

60. Singh, S.K., et al., Identification of human brain tumour initiating cells. Nature, 2004. 432(7015): p. 396-401.

61. Artavanis-Tsakonas, S., M.D. Rand, and R.J. Lake, Notch signaling: Cell fate control and signal integration in development. Science, 1999. 284(5415): p. 770-776.

62. Androutsellis-Theotokis, A., et al., Notch signalling regulates stem cell numbers in vitro and in vivo. Nature, 2006. 442(7104): p. 823-826.

63. Dexter, J.S., The Analysis of a Case of Continuous Variation in Drosophila by a Study of Its Linkage Relations. The American Naturalist, 1914. 48(576): p. $712-758$.

64. Morgan, T.H., The Theory of the Gene. The American Naturalist, 1917. 51(609): p. 513-544.

65. Poulson, D.F., Effects of Notch deficiencies. Drosophila Information Service, 1939. 12: p. 64. 
66. Lehmann, R., et al., On the phenotype and development of mutants of early neurogenesis inDrosophila melanogaster. Wilehm Roux Arch Dev Biol, 1983. 192(2): p. 62-74.

67. Greenwald, I.S., P.W. Sternberg, and H.R. Horvitz, The lin-12 locus specifies cell fates in Caenorhabditis elegans. Cell, 1983. 34(2): p. 435-44.

68. Artavanis-Tsakonas, S., M.A. Muskavitch, and B. Yedvobnick, Molecular cloning of Notch, a locus affecting neurogenesis in Drosophila melanogaster. Proc Natl Acad Sci U S A, 1983. 80(7): p. 1977-81.

69. Wharton, K.A., et al., Nucleotide sequence from the neurogenic locus notch implies a gene product that shares homology with proteins containing EGFlike repeats. Cell, 1985. 43(3 Pt 2): p. 567-81.

70. Lieber, T., et al., Antineurogenic phenotypes induced by truncated Notch proteins indicate a role in signal transduction and may point to a novel function for Notch in nuclei. Genes Dev, 1993. 7(10): p. 1949-65.

71. Rebay, I., R.G. Fehon, and S. Artavanis-Tsakonas, Specific truncations of Drosophila Notch define dominant activated and dominant negative forms of the receptor. Cell, 1993. 74(2): p. 319-29.

72. Struhl, G., K. Fitzgerald, and I. Greenwald, Intrinsic activity of the Lin-12 and Notch intracellular domains in vivo. Cell, 1993. 74(2): p. 331-45.

73. Coffman, C.R., et al., Expression of an extracellular deletion of Xotch diverts cell fate in Xenopus embryos. Cell, 1993. 73(4): p. 659-71.

74. Fitzgerald, K., H.A. Wilkinson, and I. Greenwald, glp-1 can substitute for lin-12 in specifying cell fate decisions in Caenorhabditis elegans. Development, 1993. 119(4): p. 1019-27.

75. Ellisen, L.W., et al., TAN-1, the human homolog of the Drosophila notch gene, is broken by chromosomal translocations in $T$ lymphoblastic neoplasms. Cell, 1991. 66(4): p. 649-61.

76. Weinmaster, G., V.J. Roberts, and G. Lemke, Notch2: a second mammalian Notch gene. Development, 1992. 116(4): p. 931-41.

77. Lardelli, M., J. Dahlstrand, and U. Lendahl, The novel Notch homologue mouse Notch 3 lacks specific epidermal growth factor-repeats and is expressed in proliferating neuroepithelium. Mech Dev, 1994. 46(2): p. 12336.

78. Uyttendaele, H., et al., Notch4/int-3, a mammary proto-oncogene, is an endothelial cell-specific mammalian Notch gene. Development, 1996. 122(7): p. 2251-9.

79. Kopan, R., J.S. Nye, and H. Weintraub, The intracellular domain of mouse Notch: a constitutively activated repressor of myogenesis directed at the basic helix-loop-helix region of MyoD. Development, 1994. 120(9): p. 238596.

80. Jarriault, S., et al., Signalling downstream of activated mammalian Notch. Nature, 1995. 377(6547): p. 355-8.

81. De Strooper, B., et al., Deficiency of presenilin-1 inhibits the normal cleavage of amyloid precursor protein. Nature, 1998. 391(6665): p. 387-90. 
82. Murphy, M.P. and H. LeVine, 3rd, Alzheimer's disease and the amyloid-beta peptide. J Alzheimers Dis, 2010. 19(1): p. 311-23.

83. Sherrington, R., et al., Cloning of a gene bearing missense mutations in early-onset familial Alzheimer's disease. Nature, 1995. 375(6534): p. 75460.

84. Francis, R., et al., aph-1 and pen-2 are required for Notch pathway signaling, gamma-secretase cleavage of betaAPP, and presenilin protein accumulation. Dev Cell, 2002. 3(1): p. 85-97.

85. De Strooper, B., et al., A presenilin-1-dependent gamma-secretase-like protease mediates release of Notch intracellular domain. Nature, 1999. 398(6727): p. 518-22.

86. Struhl, G. and I. Greenwald, Presenilin is required for activity and nuclear access of Notch in Drosophila. Nature, 1999. 398(6727): p. 522-5.

87. Jin, Y.H., et al., Beta-catenin modulates the level and transcriptional activity of Notchl/NICD through its direct interaction. Biochim Biophys Acta, 2009. 1793(2): p. 290-9.

88. Zhang, Y., et al., Mastermind-like transcriptional co-activator-mediated Notch signaling is indispensable for maintaining conjunctival epithelial identity. Development, 2013. 140(3): p. 594-605.

89. Traustadottir, G.A., et al., The non-canonical NOTCH1 ligand Delta-like 1 homolog (DLK1) self interacts in mammals. Int J Biol Macromol, 2017. 97: p. $460-467$.

90. Andersen, P., et al., Non-canonical Notch signaling: emerging role and mechanism. Trends Cell Biol, 2012. 22(5): p. 257-65.

91. Raya, A., et al., Notch activity acts as a sensor for extracellular calcium during vertebrate left-right determination. Nature, 2004. 427(6970): p. 121128.

92. Haines, N. and K.D. Irvine, Glycosylation regulates Notch signalling. Nat Rev Mol Cell Biol, 2003. 4(10): p. 786-97.

93. Kopan, R. and M.X. Ilagan, The canonical Notch signaling pathway: unfolding the activation mechanism. Cell, 2009. 137(2): p. 216-33.

94. Cordle, J., et al., A conserved face of the Jagged/Serrate DSL domain is involved in Notch trans-activation and cis-inhibition. Nat Struct Mol Biol, 2008. 15(8): p. 849-57.

95. Komatsu, H., et al., OSM-11 facilitates LIN-12 Notch signaling during Caenorhabditis elegans vulval development. PLoS Biol, 2008. 6(8): p. e196.

96. Warthen, D.M., et al., Jaggedl (JAG1) mutations in Alagille syndrome: increasing the mutation detection rate. Hum Mutat, 2006. 27(5): p. 436-43.

97. Guarnaccia, C., et al., The tetralogy of Fallot-associated G274D mutation impairs folding of the second epidermal growth factor repeat in Jagged-1. FEBS J, 2009. 276(21): p. 6247-57. 
98. Groot, A.J., et al., Regulated proteolysis of NOTCH2 and NOTCH3 receptors by ADAM10 and presenilins. Mol Cell Biol, 2014. 34(15): p. 282232.

99. Groot, A.J. and M.A. Vooijs, The role of Adams in Notch signaling. Adv Exp Med Biol, 2012. 727: p. 15-36.

100. Lubman, O.Y., S.V. Korolev, and R. Kopan, Anchoring notch genetics and biochemistry; structural analysis of the ankyrin domain sheds light on existing data. Mol Cell, 2004. 13(5): p. 619-26.

101. Rizzo, P., et al., Rational targeting of Notch signaling in cancer. Oncogene, 2008. 27(38): p. 5124-31.

102. Fryer, C.J., J.B. White, and K.A. Jones, Mastermind recruits $C y c C: C D K 8$ to phosphorylate the Notch ICD and coordinate activation with turnover. Mol Cell, 2004. 16(4): p. 509-20.

103. Tsunematsu, R., et al., Mouse Fbw7/Sel-10/Cdc4 is required for notch degradation during vascular development. J Biol Chem, 2004. 279(10): p. 9417-23.

104. Le Borgne, R., Regulation of Notch signalling by endocytosis and endosomal sorting. Curr Opin Cell Biol, 2006. 18(2): p. 213-22.

105. Bray, S.J., Notch signalling: a simple pathway becomes complex. Nat Rev Mol Cell Biol, 2006. 7(9): p. 678-89.

106. McGill, M.A. and C.J. McGlade, Mammalian numb proteins promote Notch1 receptor ubiquitination and degradation of the Notch1 intracellular domain. J Biol Chem, 2003. 278(25): p. 23196-203.

107. Santolini, E., et al., Numb is an endocytic protein. J Cell Biol, 2000. 151(6): p. $1345-52$.

108. Katzmann, D.J., G. Odorizzi, and S.D. Emr, Receptor downregulation and multivesicular-body sorting. Nat Rev Mol Cell Biol, 2002. 3(12): p. 893905.

109. Weber, U., C. Eroglu, and M. Mlodzik, Phospholipid membrane composition affects EGF receptor and Notch signaling through effects on endocytosis during Drosophila development. Dev Cell, 2003. 5(4): p. 55970.

110. Simons, K. and D. Toomre, Lipid rafts and signal transduction. Nat Rev Mol Cell Biol, 2000. 1(1): p. 31-9.

111. Barbieri, E., P.P. Di Fiore, and S. Sigismund, Endocytic control of signaling at the plasma membrane. Curr Opin Cell Biol, 2016. 39(Supplement C): $\mathrm{p}$. 21-7.

112. Gridley, T., Notch signaling and inherited disease syndromes. Hum Mol Genet, 2003. 12 Spec No 1: p. R9-13.

113. Morimoto, M., et al., Different assemblies of Notch receptors coordinate the distribution of the major bronchial Clara, ciliated and neuroendocrine cells. Development, 2012. 139(23): p. 4365-73.

114. Rock, J.R., et al., Notch-dependent differentiation of adult airway basal stem cells. Cell Stem Cell, 2011. 8(6): p. 639-48. 
115. Beatus, P. and U. Lendahl, Notch and neurogenesis. J Neurosci Res, 1998. 54(2): p. 125-36.

116. Fan, X., et al., Notch1 and notch2 have opposite effects on embryonal brain tumor growth. Cancer Res, 2004. 64(21): p. 7787-93.

117. Vooijs, M., et al., Mapping the consequence of Notch1 proteolysis in vivo with NIP-CRE. Development, 2007. 134(3): p. 535-44.

118. Riccio, O., et al., Loss of intestinal crypt progenitor cells owing to inactivation of both Notch1 and Notch2 is accompanied by derepression of CDK inhibitors p27Kip1 and p57Kip2. EMBO Rep, 2008. 9(4): p. 377-83.

119. van Es, J.H., et al., Notch/[gamma]-secretase inhibition turns proliferative cells in intestinal crypts and adenomas into goblet cells. Nature, 2005. 435(7044): p. 959-963.

120. Vooijs, M., Z. Liu, and R. Kopan, Notch: architect, landscaper, and guardian of the intestine. Gastroenterology, 2011. 141(2): p. 448-59.

121. Pellegrinet, L., et al., Dll1- and dll4-mediated notch signaling are required for homeostasis of intestinal stem cells. Gastroenterology, 2011. 140(4): p. 1230-1240 e1-7.

122. Korinek, V., et al., Depletion of epithelial stem-cell compartments in the small intestine of mice lacking Tcf-4. Nat Genet, 1998. 19(4): p. 379-83.

123. Pinto, D., et al., Canonical Wnt signals are essential for homeostasis of the intestinal epithelium. Genes Dev, 2003. 17(14): p. 1709-13.

124. Sainson, R.C. and A.L. Harris, Anti-Dll4 therapy: can we block tumour growth by increasing angiogenesis? Trends Mol Med, 2007. 13(9): p. 38995.

125. Patel, N.S., et al., Up-regulation of delta-like 4 ligand in human tumor vasculature and the role of basal expression in endothelial cell function. Cancer Res, 2005. 65(19): p. 8690-7.

126. Liu, Z.J., et al., Regulation of Notch1 and Dll4 by vascular endothelial growth factor in arterial endothelial cells: implications for modulating arteriogenesis and angiogenesis. Mol Cell Biol, 2003. 23(1): p. 14-25.

127. Suzuki, M., et al., Delta-4 expression on a stromal cell line is augmented by interleukin-6 via STAT3 activation. Exp Hematol, 2006. 34(9): p. 1143-50.

128. Seo, S., et al., The forkhead transcription factors, Foxcl and Foxc2, are required for arterial specification and lymphatic sprouting during vascular development. Dev Biol, 2006. 294(2): p. 458-70.

129. Shawber, C.J., et al., Notch signaling in primary endothelial cells. Ann N Y Acad Sci, 2003. 995: p. 162-70.

130. Diez, H., et al., Hypoxia-mediated activation of Dll4-Notch-Hey2 signaling in endothelial progenitor cells and adoption of arterial cell fate. Exp Cell Res, 2007. 313(1): p. 1-9.

131. Rangarajan, A., et al., Notch signaling is a direct determinant of keratinocyte growth arrest and entry into differentiation. EMBO J, 2001. 20(13): p. 342736. 
132. Han, H., et al., Inducible gene knockout of transcription factor recombination signal binding protein-J reveals its essential role in $T$ versus B lineage decision. Int Immunol, 2002. 14(6): p. 637-45.

133. Radtke, F., et al., Deficient $T$ cell fate specification in mice with an induced inactivation of Notch1. Immunity, 1999. 10(5): p. 547-58.

134. Oh, P., et al., In vivo mapping of notch pathway activity in normal and stress hematopoiesis. Cell Stem Cell, 2013. 13(2): p. 190-204.

135. Garg, V., et al., Mutations in NOTCH1 cause aortic valve disease. Nature, 2005. 437(7056): p. 270-4.

136. Marathe, S., et al., Jaggedl Is Altered in Alzheimer's Disease and Regulates Spatial Memory Processing. Front Cell Neurosci, 2017. 11: p. 220.

137. Sharma, A., et al., A novel Monoclonal Antibody against Notch1 Targets Leukemia-associated Mutant Notchl and Depletes Therapy Resistant Cancer Stem Cells in Solid Tumors. Sci Rep, 2015. 5: p. 11012.

138. Westhoff, B., et al., Alterations of the Notch pathway in lung cancer. Proc Natl Acad Sci U S A, 2009. 106(52): p. 22293-8.

139. Hassan, K.A., et al., Notch pathway activity identifies cells with cancer stem cell-like properties and correlates with worse survival in lung adenocarcinoma. Clin Cancer Res, 2013. 19(8): p. 1972-80.

140. Theys, J., et al., High NOTCH activity induces radiation resistance in non small cell lung cancer. Radiother Oncol, 2013. 108(3): p. 440-445.

141. Wang, N.J., et al., Loss-of-function mutations in Notch receptors in cutaneous and lung squamous cell carcinoma. Proc Natl Acad Sci U S A, 2011. 108(43): p. 17761-6.

142. Matsuno, K., et al., Deltex acts as a positive regulator of Notch signaling through interactions with the Notch ankyrin repeats. Development, 1995. 121(8): p. 2633-44.

143. Huber, R.M., et al., Deltex-1 activates mitotic signaling and proliferation and increases the clonogenic and invasive potential of U373 and LN18 glioblastoma cells and correlates with patient survival. PLoS One, 2013. 8(2): p. e57793.

144. Purow, B.W., et al., Notch-1 regulates transcription of the epidermal growth factor receptor through p53. Carcinogenesis, 2008. 29(5): p. 918-25.

145. Brennan, C., et al., Glioblastoma subclasses can be defined by activity among signal transduction pathways and associated genomic alterations. PLoS One, 2009. 4(11): p. e7752.

146. Wang, J., et al., Notch promotes radioresistance of glioma stem cells. Stem Cells, 2010. 28(1): p. 17-28.

147. Fan, X., et al., NOTCH pathway blockade depletes CD133-positive glioblastoma cells and inhibits growth of tumor neurospheres and xenografts. Stem Cells, 2010. 28(1): p. 5-16.

148. Yahyanejad, S., et al., NOTCH blockade combined with radiation therapy and temozolomide prolongs survival of orthotopic glioblastoma. Oncotarget, 2016. 7(27): p. 41251-41264. 
149. Aster, J.C., W.S. Pear, and S.C. Blacklow, The Varied Roles of Notch in Cancer. Annu Rev Pathol, 2017. 12(1): p. 245-275.

150. Wang, K., et al., PEST domain mutations in Notch receptors comprise an oncogenic driver segment in triple-negative breast cancer sensitive to a gamma-secretase inhibitor. Clin Cancer Res, 2015. 21(6): p. 1487-96.

151. Weng, A.P., et al., Activating mutations of NOTCH1 in human T cell acute lymphoblastic leukemia. Science, 2004. 306(5694): p. 269-71.

152. Song, J.H., et al., FBXW7 mutation in adult T-cell and B-cell acute lymphocytic leukemias. Leuk Res, 2008. 32(11): p. 1751-5.

153. Babaei-Jadidi, R., et al., FBXW7 influences murine intestinal homeostasis and cancer, targeting Notch, Jun, and DEK for degradation. J Exp Med, 2011. 208(2): p. 295-312.

154. Cheng, Y. and G. Li, Role of the ubiquitin ligase Fbw7 in cancer progression. Cancer Metastasis Rev, 2012. 31(1-2): p. 75-87.

155. Gallahan, D., C. Kozak, and R. Callahan, A new common integration region (int-3) for mouse mammary tumor virus on mouse chromosome 17. J Virol, 1987. 61(1): p. 218-20.

156. Gallahan, D. and R. Callahan, The mouse mammary tumor associated gene INT3 is a unique member of the NOTCH gene family (NOTCH4). Oncogene, 1997. 14(16): p. 1883-90.

157. Zhu, J., et al., Deciphering genomic alterations in colorectal cancer through transcriptional subtype-based network analysis. PLoS One, 2013. 8(11): p. e79282.

158. Zhang, Q., et al., Fbxw7 Deletion Accelerates Kras(G12D)-Driven Pancreatic Tumorigenesis via Yap Accumulation. Neoplasia, 2016. 18(11): p. 666-673.

159. Bailey, P., et al., Genomic analyses identify molecular subtypes of pancreatic cancer. Nature, 2016. 531(7592): p. 47-52.

160. Cha, S., et al., Clinical application of genomic profiling to find druggable targets for adolescent and young adult (AYA) cancer patients with metastasis. BMC Cancer, 2016. 16: p. 170.

161. Guo, Y., et al., Numb(-/low) Enriches a Castration-Resistant Prostate Cancer Cell Subpopulation Associated with Enhanced Notch and Hedgehog Signaling. Clin Cancer Res, 2017. 23(21): p. 6744-6756.

162. Stoyanova, T., et al., Activation of Notch1 synergizes with multiple pathways in promoting castration-resistant prostate cancer. Proc Natl Acad Sci U S A, 2016. 113(42): p. E6457-E6466.

163. Killela, P.J., et al., The genetic landscape of anaplastic astrocytoma. Oncotarget, 2014. 5(6): p. 1452-7.

164. Ling, H., J.R. Sylvestre, and P. Jolicoeur, Notchl-induced mammary tumor development is cyclin D1-dependent and correlates with expansion of premalignant multipotent duct-limited progenitors. Oncogene, 2010. 29(32): $\mathrm{p}$. 4543-4554. 
165. Cohen, B., et al., Cyclin D1 is a direct target of JAG1-mediated Notch signaling in breast cancer. Breast Cancer Res Treat, 2010. 123(1): p. 11324.

166. $\mathrm{Xu}, \mathrm{P}$., et al., The oncogenic roles of Notchl in astrocytic gliomas in vitro and in vivo. J Neurooncol, 2010. 97(1): p. 41-51.

167. Armstrong, F., et al., NOTCH is a key regulator of human T-cell acute leukemia initiating cell activity. Blood, 2009. 113(8): p. 1730-40.

168. Fan, X., et al., Notch pathway inhibition depletes stem-like cells and blocks engraftment in embryonal brain tumors. Cancer Res, 2006. 66(15): p. 744552.

169. Harrison, H., et al., Breast cancer stem cells: something out of notching? Cancer Res, 2010. 70(22): p. 8973-6.

170. Sikandar, S.S., et al., NOTCH signaling is required for formation and selfrenewal of tumor-initiating cells and for repression of secretory cell differentiation in colon cancer. Cancer Res, 2010. 70(4): p. 1469-78.

171. Nicolas, M., et al., Notchl functions as a tumor suppressor in mouse skin. Nat Genet, 2003. 33(3): p. 416-21.

172. Demehri, S., A. Turkoz, and R. Kopan, Epidermal Notch1 loss promotes skin tumorigenesis by impacting the stromal microenvironment. Cancer Cell, 2009. 16(1): p. 55-66.

173. Vliet-Gregg, P.A., J.R. Hamilton, and R.A. Katzenellenbogen, Human papillomavirus 16E6 and NFX1-123 potentiate Notch signaling and differentiation without activating cellular arrest. Virology, 2015. 478: p. 5060.

174. Agrawal, N., et al., Exome sequencing of head and neck squamous cell carcinoma reveals inactivating mutations in NOTCH1. Science, 2011. 333(6046): p. 1154-7.

175. South, A.P., et al., NOTCH1 mutations occur early during cutaneous squamous cell carcinogenesis. J Invest Dermatol, 2014. 134(10): p. 26302638.

176. Rampias, T., et al., A new tumor suppressor role for the Notch pathway in bladder cancer. Nat Med, 2014. 20(10): p. 1199-205.

177. Song, Y., et al., Identification of genomic alterations in oesophageal squamous cell cancer. Nature, 2014. 509(7498): p. 91-5.

178. Samon, J.B., et al., Preclinical analysis of the gamma-secretase inhibitor PF-03084014 in combination with glucocorticoids in T-cell acute lymphoblastic leukemia. Mol Cancer Ther, 2012. 11(7): p. 1565-75.

179. Real, P.J. and A.A. Ferrando, NOTCH inhibition and glucocorticoid therapy in T-cell acute lymphoblastic leukemia. Leukemia, 2009. 23(8): p. 1374-7.

180. Real, P.J., et al., Gamma-secretase inhibitors reverse glucocorticoid resistance in T cell acute lymphoblastic leukemia. Nat Med, 2009. 15(1): p. 50-8.

181. Nowell, C.S. and F. Radtke, Notch as a tumour suppressor. Nat Rev Cancer, 2017. 17(3): p. 145-159. 


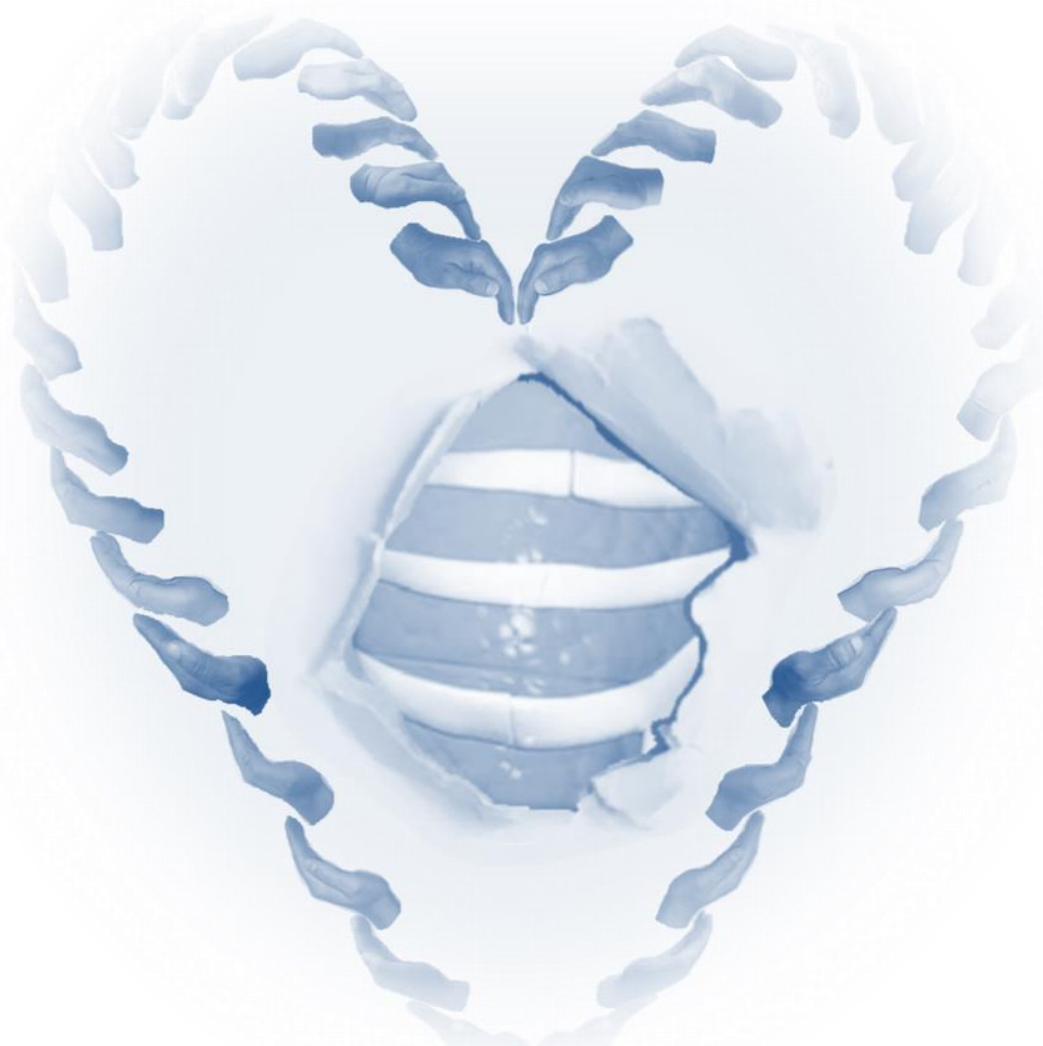




\section{CHAPTER 2}

\section{Drug resistance in non-small cell lung cancer: a potential for NOTCH targeting?}

Venus Sosa Iglesias, Lorena Giuranno, Ludwig J Dubois*, Jan Theys*, Marc Vooijs.

*Contributed equally

Department of Radiation Oncology

GROW - School for Oncology \& Developmental Biology

Maastricht University Medical Center (MUMC)

Maastricht, the Netherlands

Submitted to Frontiers in Oncology 


\section{TABLE OF CONTENTS}

LUNG CANCER AND STANDARD OF CARE

THE LUNG CANCER GENOME: ACTIONABLE TARGETS IN NSCLC?

Lung CANCER STEM CELLS

THE CANONICAL NOTCH SIGNALING PATHWAY

NOTCH in a Physiological \& PAThological CONTEXT IN THE LUNG

ONCOGENE ADDition AND TREATMENT RESISTANCE

NOTCH-RELATED RESISTANCE TO CHEMOTHERAPY

NOTCH-RELATED RESISTANCE TO TARGETED THERAPIES

NOTCH TARGETING IN NSCLC

PRECLINICAL EFFECTS OF NOTCH INHIBITORS

CLINICAL TRIALS USING NOTCH PATHWAY INHIBITORS

60

SIDE-EFFECTS OF NOTCH THERAPEUTICS

NOTCH INHIBITION IN LUNG CANCER COMORBIDITIES

Conclusions ANd Perspectives

LITERATURE AND SEARCH CRITERIA

ACKNOWLEDGEMENTS

FIGURE AND TABLES 


\section{Abstract}

Drug resistance is a major cause for therapeutic failure in non-small cell lung cancer (NSCLC) leading to tumor recurrence, and disease progression. Cell intrinsic mechanisms of resistance include changes in the expression of drug transporters, activation of pro-survival and anti-apoptotic pathways, as well non-intrinsic influences of the tumor microenvironment. It has become evident that tumors are composed of a heterogeneous population of cells with different genetic, epigenetic, and phenotypic characteristics that results in diverse responses to therapy and underlies the emergence of resistant clones. This tumor heterogeneity is driven by subpopulations of tumor cells termed cancer stem cells (CSCs) that have tumor initiating capabilities, are highly self-renewing, and retain the ability for multilineage differentiation. CSCs have been identified in NSCLC and have been associated with chemo- and radiotherapy resistance. Stem cell pathways are frequently deregulated in cancer and are implicated in recurrence after treatment. Here we focus on the Notch signaling pathway, which has a role in stem cell maintenance, in non-squamous non-small lung cancer, and we critically assess the potential for targeting the Notch pathway to overcome resistance to chemotherapeutic and targeted agents using both preclinical and clinical evidence.

\section{Lung cancer and Standard of care}

According to the World Health Organization, in 2015, every 3.5 seconds a person died of cancer and one out of five deaths were due to lung cancer. Lung cancer is the second most common type of cancer and the leading cause of cancer-related mortality. More than two thirds of lung cancer patients are diagnosed at an advance stage (III-IV). The lack of early diagnostic techniques, and the intrinsic and/or acquired treatment resistance leading to relapse are major obstacles in finding a cure for lung cancer.

Lung cancer can be divided into two main categories: non-small cell lung cancer (NSCLC) accounting for $85 \%$ of lung cancers, and small cell lung cancer ( 15\%). NSCLC can be further categorized into, generally, adenocarcinoma (AC 40\%), squamous cell carcinoma (SQCC 25-30\%), large cell undifferentiated carcinoma (10-15\%), mixed subtypes (adenosquamous), and the far less common sarcomatoid carcinoma. Treatment for NSCLC consists of surgical resection, chemotherapy, radiation, targeted therapy, and/or combinations thereof. Standard first line treatment 
for inoperable locally advanced stage NSCLC cancer is concurrent polychemotherapy with fractionated radiation (60 Gy in 2 Gy fractions) [1]. Studies show that chemoradiotherapy (using paclitaxel) as opposed to radiotherapy alone, delivered after induction chemotherapy (carboplatin and paclitaxel), is feasible and improves time to progression and overall survival of inoperable stage III NSCLC [2].

Polychemotherapy for NSCLC often involves the combination of a platinum-based agent (e.g. cisplatin, carboplatin) and other drugs with different mechanism of action. Cisplatin or carboplatin covalently binds DNA and activates the DNA-damage response, induces cell cycle arrest, and apoptosis. The second chemotherapeutic agent can be a topoisomerase II inhibitor (e.g. etoposide), a DNA damaging agent preventing replication such as a taxane (e.g. paclitaxel, docetaxel) or a vinca-alkaloid (e.g. vincristine, vinorelbine, vinblastine) which inhibits microtubule assembly and blocks mitosis, an altered DNA base that gets incorporated in the DNA but cannot be repaired (e.g. gemcitabine), or an inhibitor of folate metabolism (e.g. pemetrexed). There are studies that suggest that the selection of the chemotherapeutic agent should consider the subtype of NSCLC. Second-line paclitaxel treatment for cisplatintreated lung cancer patients benefits clinical outcome (response rate plus stable disease) in non-squamous cell carcinomas preferentially [3]. Adenocarcinoma seems to have better overall survival rates for both gemcitabine-platinum and taxaneplatinum regimens, where the first, gives better objective response rates and a tendency to improved median survival time (9.1 versus 7.4 months in the taxane combination) [4]. Squamous cell carcinoma patients could benefit more from a cisplatin plus etoposide treatment rather than the four-drug combination: cyclophosphamide, adriamycin, methotrexate, and procarbazine where the response rate is $44.7 \%$ versus $21.6 \%$ respectively [5]. Large cell neuroendocrine carcinomas and small cell lung carcinomas have a similar biological behavior and respond similarly to some treatments including: irinotecan, platinum, and taxanes, which are more effective than pemetrexed [6-8]. The remaining subtypes of large cell carcinomas (non-neuroendocrine), sarcomatoid tumors, and mixed carcinomas often don't have well defined biological features, the criteria for diagnosis are not as robust, and hence, treatment response has not been properly assessed. Regarding targeted agents, several studies suggest that adenocarcinoma patients benefit more from EGFR-TKI therapy than squamous cell carcinoma patients, both subtypes bearing EGFR mutations, where objective response rates, overall survival, and progression-free survival are 66-74\%, 19-21 months, and 9.4-10 months [9] versus 25-27\%, 13.48 months, and 3-5 months [10, 11], respectively. Within the adenocarcinoma subtypes, the brochioloalveolar one is the most responsive to small 
molecule tyrosine kinase inhibitors (e.g. gefitinib) [12]. These observations raise the following question: "which are the reasons behind these diverse responses and outcomes, to the same treatments, between lung cancer subtypes and patients?"

\section{The lung cancer genome: Actionable targets in NSCLC?}

Whole genome sequencing of lung cancers has revealed complex patterns of driver mutations with over 200 non-synonymous mutations that distinguish smokers from non-smokers and predict patient outcome [13-15]. Mutations in KRAS occur in up to $25 \%$ of NSCLC and despite preclinical efforts, there are no clinically approved drugs that effectively target KRAS. In lung adenocarcinoma, actionable mutations in the epidermal growth factor receptor (EGFR) occur with a 10-15\% frequency and can be effectively targeted with small molecule first- and second-generation tyrosine kinase inhibitors (TKI) (e.g. erlotinib, gefitinib, afatinib), and monoclonal antibodies (e.g. cetuximab). TKI that target translocations in the anaplastic lymphoma kinase (EML4-ALK), occurring with a $5 \%$ frequency in adenocarcinomas, are also available (e.g. ceritinib, alectinib, crizotinib). Other actionable driver mutations $(\sim 15-20 \%)$ that occur less commonly are ROS1 rearrangements, BRAF mutations, RET rearrangements, NTRK1 rearrangements, MET amplifications and HER2 mutations. In about $40 \%$ of lung adenocarcinomas however, there are no common driver genes yet identified [16]. High response rates (60-70\%) are achieved with the EGFR TKIs in EGFR-mutated cancers [9] and $~ 60 \%$ of partial/complete responses with ALK inhibitors (e.g. crizotinib) in patients with ALK translocations [17]. However, resistance to pharmacological inhibitors, for example TKIs, seems inevitable. Mechanisms of resistance include alteration of the drug target such as resistance mutations, alternative splicing, and gene amplification, as well as activation of alternative oncogenic pathways. Tumor cells which harbor these resistance-creating mutations can be present at the onset of treatment (primary) or emerge during treatment (secondary resistance). Other mechanisms of resistance, for instance inefficient drug delivery, metabolic inactivation and drug-interactions, also play a role in therapeutic outcome. The most frequent form of acquired resistance in NSCLC are secondary mutations in EGFR (e.g. T790M 'gatekeeper') occurring in $60 \%$ of patients treated with $2^{\text {nd }}$ generation TKIs. Similarly, secondary mutations in ALK (e.g. C1156Y, L1196M, G1269A, L1152R) are associated with acquired resistance to first generation ALK inhibitors such as crizotinib. In addition, there are several pathways that can mediate resistance to TKI which include the activation of anti-apoptotic pathways, HER2 and MET amplification, or mutations in PIK3CA or BRAF [18]. 
In squamous cell carcinoma subtype of non-small cell lung cancers (SQCC NSCLC), most tumors carry mutations in TP53, RB1, CDKN2A and in the oxidative pathway genes KEAP and NFE2L2. EGFR and ALK mutations, common in adenocarcinomas, are less frequent in SQCC of the lung and hence, agents developed for lung adenocarcinoma are less effective against lung SQCC. In adenocarcinoma patients, EGFR-TKI objective response rates, overall survival, and progression-free survival are 66-74\%, 19-21 months, and 9.4-10 months [9] versus 25-27\%, 13.48 months, and 3-5 months for SQCC [11, 19], respectively. Interestingly, SQCC differentiation genes such as SOX2 and TP63 (TP53 homolog) are commonly altered and mutually exclusive with loss of function mutations in NOTCH1 and NOTCH2. Other alterations include amplification of EGFR, FGFR1, and PI3K pathways [20]. Alternative approaches that target the tumor microenvironment using antiangiogenic therapies such as antibodies or small molecule inhibitors aimed at the vascular endothelial growth factor (VEGF) or its receptor (VEGFR), were the first targeted agents to yield a significant improvement in overall survival when combined with first-line chemotherapy for metastatic NSCLC. Anti-angiogenic treatment however, also resulted in strong normal tissue toxicities [21]. Importantly, antiangiogenesis inhibition combined with platinum chemotherapy does not improve outcome for squamous NSCLC [22].

Remarkable progression-free survival rates have been observed in advanced NSCLC using checkpoint inhibitors (e.g. Nivolumab, Ipilimumab) as first line treatment, superior to chemotherapy in both squamous and non-squamous NSCLC [23]. Checkpoint inhibitors that target PD-L1/PD-1 and CTL-A4 receptors expressed on immune and tumor cells, block the anti-tumor adaptive immune-response by suppressing the cytotoxic T-cell response. Monoclonal antibodies that block the interaction between PD-L1 and PD-1 (e.g. Durvalumab) improve progression-free survival (16.8 vs 5.6 months (placebo)), the response rate and its duration (72.8\% versus $46.8 \%$ 18-month response) in stage III unresectable NSCLC pre-treated with platinum-based chemotherapy [24, 25]. There are still many factors that remain uncertain that would enable clinicians to determine the response to checkpoint inhibitors but a high mutation load creates immunogenic tumors and is strongly associated with response to checkpoint inhibitors [26]. Unfortunately, most NSCLC patients do not respond to such immunotherapies despite expressing PD-L1 and the disease progresses, indicative of resistance to checkpoint inhibitors [27].

Better and more holistic approaches have been proposed showing that a "cancer mutation signature" is more predictive for treatment response than the individual 
mutation status [28]. In KRAS-driven NSCLC, the signature: FOXRED2, KRAS, TOP1, PEX3 and ABL2 was more predictive for prognosis than the single mutation status of KRAS [29]. An RNA-seq-based prognostic model built with four genes (RHOV, CD109, FRRS1 and LINC00941) was statistically associated with worse overall survival and metastasis-free survival and is able to stratify patients bearing KRAS or EGFR mutations versus their wild type counterparts in overall survival outcome [30]. Because lung cancer is a highly heterogeneous disease on the genetic, epigenetic and metabolic levels, it is perhaps not so surprising that personalized medical approaches targeting only one driver mutation improve overall survival but cannot increase cure rates.

\section{Lung cancer heterogeneity}

Cancers are composed of mixed cell populations with diverse genotypic, epigenetic, phenotypic, and morphological characteristics. Tumor heterogeneity is observed among different patients with the same tumor subtype (interpatient heterogeneity), among tumor cells within one host organ (intratumor heterogeneity), between the primary and the metastatic tumors (intermetastatic heterogeneity), and among tumor cells within the metastatic site (intrametastatic heterogeneity) [31]. It was first exemplified in renal cancer that biopsies from primary and metastatic sites from the same patient showed extensive divergent and convergent evolution of driver mutations, copy number variations, and chromosome aneuploidy [32]. It is has been proposed for a long time now that these subclonal tumor populations, present at low frequency, contain clones with invasive and metastatic properties [33], and escape mechanisms for systemic and targeted treatments that affect clinical outcome. It is well understood that heterogeneity is not only determined by cell intrinsic mechanisms but also by the dynamic tumor microenvironment (e.g. angiogenesis, immune system, fibroblasts) [34]. Lung cancer is also highly heterogeneous with respect to metabolic activity and blood perfusion at the macro-level as well as at the single-cell level [35, 36]. Genome sequencing in NSCLC has identified hundreds of mutations present in subclonal fractions that increase with tumor-grade [15, 37], and in primary tumors, predict early postsurgical relapse [38]. Smokers have 10-fold more mutations than non-smokers and distinct driver mutations (e.g. EGFR vs KRAS) [14, 15]. Chromosomal instability which is a driver intra-tumor heterogeneity, is associated with cancer drug resistance and associated with poor outcome in NSCLC. Tumor subclones have different actionable therapeutic targets explaining the variety of responses to targeted therapeutics $[39,40]$. In addition to the genetic and epigenetic heterogeneity, there is a high degree of heterogeneity in 
tumor metabolism which is highly dynamic and subject to changes in oxygen, nutrients and other tumor microenvironmental factors [35]. Taken together, the different levels of heterogeneity in tumors are of high clinical relevance in tumor progression, treatment response, and relapse. One of the main genetic drivers of tumor heterogeneity is cancer stem cells which maintain a tumor cell hierarchy [41].

\section{Lung Cancer Stem cells}

Cancer stem cells (CSC) were first identified in myeloid leukemias by Dick and colleagues. CSC are tumor initiating cells responsible for the cellular hierarchy maintained by means of self-renewal, and causing tumor heterogeneity, and are capable of multipotent differentiation [42]. Tumor heterogeneity may also be due to the plasticity of cancer stem cells which enables them to differentiate reversibly into different cell types under specific environmental conditions [43]. Furthermore, differentiated cancer cells may be reprogrammed to a more stem-cell like state under specific conditions (e.g. hypoxia-induced OCT-4 and Nanog) [44] and hence, contribute to recurrence. Additionally, chromosomal instability together with external environmental factors, may lead to cancer stem cell heterogeneity and even to metastasis.

Cancer stem cells from NSCLC have the ability to form colonies in soft-agar, they are highly tumorigenic in vivo [45], can be identified by virtue of Hoechst dye-efflux (the side population, SP) using flow cytometric methods. CSCs express multidrug $\mathrm{ABC}$-transporters and are resistant to multiple chemotherapeutic agents [46]. One of the best-characterized cancer stem cell markers for solid cancer, including NSCLC, is the CD133 cell surface protein. CD133 expressing (CD133+) lung cancer cells are self-renewing tumor cells that express markers from embryonic stem cells, are present in low numbers in human NSCLC but are highly tumorigenic. Moreover, when CD133+ CSC differentiate, their CD133- progeny is no longer tumorigenic [47]. It seems plausible, that combination therapy targeting dually and specifically stem cells and non-stem cells, would be required to be successful in, or at least be closer to, eradicating cancer [48]. There is now mounting evidence that the normal stem cell pathways such as Wnt, Notch and Hh (Hedgehog) are deregulated and mutated in cancer and cancer stem cells [49]. NOTCH signaling plays a role in the maintenance of cancer stem cells in different cancer types including T-ALL [50], brain [51], breast [52], colon [53] and lung cancer [54]. 


\section{The canonical NOTCH signaling pathway}

The NOTCH signaling pathway is a highly conserved cell-to-cell communication pathway between cells expressing the single pass transmembrane NOTCH receptor and neighboring cells expressing a transmembrane NOTCH ligand. It is a major cell fate determination pathway essential for embryonic development. In adult tissues, NOTCH signaling regulates tissue homeostasis through cell renewal, differentiation, proliferation and cell death $[55,56]$. The mammalian genome encodes for four NOTCH genes (NOTCH 1-4) and five NOTCH ligands (Jagged1, 2 or Delta1, 3 and 4). NOTCH signaling occurs at the cell surface and is highly regulated by the proteolytic cleavage of the NOTCH receptor. NOTCH receptors are transported to the cell-surface as furin-cleaved heterodimers and ligand interaction initiates two consecutive proteolytic cleavages. The first proteolytic cleavage is executed by the ADAM10 metalloprotease which cleaves the NOTCH ectodomain and is followed by the intramembranous and rate-limiting cleavage by the $\gamma$-secretase complex [57]. The $\gamma$-secretase liberates the NOTCH Intracellular Domain (NICD) which then translocates to the nucleus where it binds to the DNA-bound protein CSL (also called RBP-Jk), and together with the Mastermind (MAML) co-activators, forms the NOTCH transcriptional complex. In the C-terminal end of the NICD, there is a proline/glutamic acid/serine/threonine-rich motif (PEST) which is a substrate for the E3 ubiquitin ligase Fbwx7 and targets NICD for proteasomal degradation when the signal needs to be shut down.

NOTCH regulates the transcription of genes of the HES and HEY family, CD25 and GATA3 (in T cells), negative regulators of NOTCH signaling (NRARP, Deltex1), oncogenes like RAS, cyclin D1, p21/Waf1, and c-MYC, among many others [58]. $\mathrm{NOTCH}$ signaling has been found deregulated in multiple human diseases, and recently, there is growing evidence supporting the role of NOTCH signaling in the development and progression of cancers [59]. Gain-of-function mutations are a hallmark in T-cell acute lymphoblastic leukemia (T-ALL) [60], but overexpression and mutations in NOTCH pathway members are found at lower frequencies in many other leukemias and solid cancers. A recent review summarized the involvement of Notch signaling in all acquired capabilities of cancer cells already defined by Hanahan and Weinberg as the hallmarks of cancer [61, 62]. 


\section{NOTCH in a Physiological \& Pathological context in the Lung}

NOTCH receptors and ligands are expressed during early lung development and control cell fate specification and branching along the proximal-distal axis [63, 64]. NOTCH blockade reduces the number of SOX2 progenitors and alters the balance between basal, ciliated, neuroendocrine and secretory cell fates in the airway epithelium $[65,66]$. In the postnatal lung, NOTCH restricts basal cells to the secretory cell fate suppressing ciliated differentiation [67]. NOTCH signaling is also required to maintain the differentiated state of secretory cells in the upper airways and blocking JAGGED1/2-NOTCH signaling leading to transdifferentiation of club cells into ciliated cells [68]. NOTCH2 regulates differentiation of lineage-restricted progenitors into bronchial club cells and ciliated cells as well as contributing to alveolar morphogenesis and integrity of epithelial and smooth muscle layers of airways $[69,70]$. NOTCH1/3 contribute additively to regulate pulmonary neuroendocrine cell fate [70]. While NOTCH1 is dispensable for airway epithelial development upon lung epithelial injury, NOTCH1 is essential to induce club cell regeneration by activating its downstream targets Hes5 and Pax6 [71].

Deregulation or mutation of NOTCH receptors, ligands, and signaling regulators, is associated with pathogenesis of many hematological and solid tumors including lung cancer [72]. In T-cell leukemias, NOTCH1 activating mutations occur in $60 \%$ of cases. Many human lung cancer cell lines (20\%), and primary lung cancers, harbor missense or non-sense mutations in one of the NOTCH receptors [73]. Translocations involving NOTCH3 were first identified in NSCLC [74], are found overexpressed in $30 \%$ of NSCLC, and are strongly correlated with EGFR expression [75]. Gain of function mutations in NOTCH1 or loss of the negative NOTCH regulator NUMB have been identified in up to $30 \%$ of adenocarcinomas and are correlated with poor prognosis. Loss of NUMB is also correlated with higher NOTCH activity, and in tumors with wild type TP53 NOTCH1 expression, it was associated with worse outcome [76]. Furthermore, high NOTCH1 and NOTCH3 receptors, ligand, and target gene expression, are correlated with worse survival in resected NSCLC $[54,77,78]$. Other studies have shown conflicting data on the role of NOTCH1 expression and its influence on the outcome in NSCLC [79, 80]. It must be noted however, that lung cancers were not sub-classified into adenocarcinoma and squamous carcinoma in all studies. A meta-analysis confirmed positive correlations of NOTCH1 and 3 expressions with progression and overall survival (OS) in adenocarcinoma (but not in lung SQCC) however, this was only significant for NOTCH3 [81]. For the NOTCH target gene HES1 significant associations between 
expression and overall survival have been found in adenocarcinoma [54, 78, 81]. In addition to its oncogenic role, inactivating mutations in NOTCH receptors has also been associated with squamous cancers of the skin, head and neck, and lung [82]. The tumor suppressive role of NOTCH in epidermal differentiation was first identified in mice with keratinocyte-specific loss of Notch1 which developed skin carcinoma [83]. More recently, sequencing analysis has identified missense and nonsense mutations in SQCC in NOTCH1 or 2 that suggest a loss of function [84, 85 ] but no loss-of-function mutations have been reported for NOTCH ligands or target genes.

\section{Oncogene Addition and Treatment Resistance}

In 2002, Weinstein proposed a potential Achilles heel of cancer which he referred to as oncogene addiction, whereby the expression of oncogenes is not only required for the initiation of tumorigenesis but also for the maintenance of the malignant phenotype [86]. This concept was coined based on findings from preclinical studies in which tumors regressed when c-MYC, KRAS, TP53 and other commonly mutated oncogenes that were used to initiate the tumors, were turned off. Unfortunately, two decades later there are still no clinically approved therapeutics against MYC, RAS or TP53. To date, the only pharmacological proof of oncogene addiction in patients is in chronic myelogenous leukemia (CML) were tumors regress and are cured upon targeting the BCR-Abl fusion gene with the small molecule inhibitor serine/threonine kinase inhibitor Gleevec. In order to survive, tumor cells evolve by either promoting the emergence of new tumor clones that are no longer dependent on the initial activating oncogene (primary resistance), or by developing mutations (of the drug target or downstream activating mutations) that make tumors that were initially responders, insensitive to monotherapy treatment (secondary resistance). For example, during anti-EGFR treatment (e.g. Cetuximab) of metastatic colorectal cancer, KRAS mutant cells can be identified in the blood of patients while tumors are still regressing [87]. Despite the paramount clinical success of Gleevec, resistance also develops by acquisition of mutations in the binding site of Gleevec [88]. It is evident that monotherapies of specific targeted agents will not lead to cancer cure, therefore drug combinations are required. Given the important role of Notch signaling in cancer stem cells and its frequent involvement in NSCLC we questioned whether NOTCH targeting combined with systemic chemotherapy or targeted treatments is a promising direction to pursue. 


\section{NOTCH-related Resistance to Chemotherapy}

\section{Platinum-based drugs (cisplatin, carboplatin)}

Platinum-based drugs bind covalently to DNA thereby interfering with replication, particularly in fast-growing cells, and prompt activation of DNA-damage recognition and repair mechanisms leading to cell cycle arrest or apoptosis when repair is not effective. In vitro and in vivo studies show that cisplatin enriches a subpopulation of NOTCH-regulated CD133expressing stem-like lung cancer cells that cause crossresistance to paclitaxel and doxorubicin by upregulation of ABCG2 and ABCB1 (also called MDR-1 or P-glycoprotein), ATP-binding cassette (ABC) drug transporters [89]. Besides CD133 expression, NSCLC cells expressing CD44, Nanog, Oct-4, SOX-2, and ALDHA1 were shown to be resistant to cisplatin through NOTCH3-mediated activation of autophagy [90]. Lung cancer stem cells downregulate $\mathrm{AQP} 2$ and CTR1 drug transporter genes, consequently leading to reduced drug uptake and intracellular accumulation, increased DNA damage, and resistance to treatment. Moreover, these cells display an increased ability to repair cisplatin-induced DNA intrastrand cross-links via activation of nucleotide-excision and mismatch repair pathways [91].

Cisplatin-resistant stem-like cells also display upregulated EMT (EpithelialMesenchymal transition) markers [92]. EMT is physiologically important during embryogenesis involving the loss of cell-to-cell junctions, loss of epithelial (e.g. Ecadherin/CDH1) and gain of mesenchymal markers (e.g. N-cadherin, Vimentin). Pathologically it is involved in tumorigenesis, metastasis and therapeutic resistance. NOTCH induces EMT via activation of transcription factors including TWIST, SNAIL, SLUG, and ZEB [93]. Additionally, NOTCH expression has been shown to be regulated by certain growth factors involved in EMT including FGF (Fibroblast growth factor) and PDGF (Platelet-derived growth factor) [94].

Poor prognosis of NSCLC patients with activated NOTCH signaling (either by $\mathrm{NOTCH}$ receptor activating mutations or loss of NUMB repressor) has been associated with absence of mutations in the tumor suppressor protein TP53 [76]. Aberrantly high TP53 expression before the start of treatment is associated with strong histopathological responses (e.g. necrosis, fibrosis) to cisplatin, and it has been reported that in only $13 \%$ of the cases, there is an alteration of TP53 expression levels before and after cisplatin treatment in stage IIIA NSCLC [95]. Interestingly, 
NUMB, a suppressor of NOTCH, forms a tri-complex with TP53 and its ubiquitin ligase HDM2 to prevent ubiquitination and consequent TP53 degradation [96]. Therefore, in cancers with loss of NUMB, such as some breast cancers and lung adenocarcinomas, there is an increase in NOTCH receptor and a decrease in TP53 protein expression levels thus enhancing chemoresistance. Additionally, CSL/RBP$\mathrm{Jk}$, a DNA binding protein that mediates NOTCH transcriptional activation, can be negatively regulated by TP53 which in turn can decrease CSL expression as feedback inhibition [97]. There is evidence that NOTCH3 signaling in ovarian cancer is also predictive for platinum resistance [98]. NOTCH3 signaling is active in drug-resistant cancer stem cells and NOTCH3 inhibition induces chemosensitivity to platinumbased drugs. The preclinical and clinical data here discussed suggests that not only platinum-sensitive but also platinum-resistant cancers may benefit from NOTCH targeting. However, whether NOTCH targeting induces platinum sensitivity in NSCLC patients is not known.

\section{Microtubule-targeting inhibitors (Taxanes, Vinca Alkaloids)}

Taxanes (docetaxel, paclitaxel) and Vinca alkaloids (vinblastine, vincristine, vinorelbine) interfere with microtubule function by preventing either depolymerization (taxanes) or microtubule formation (Vinca alkaloids), and ultimately blocking cell cycle progression through mitosis. Overexpression of ABC drug transporters (ABCB1/MDR-1/P-gp) mediates resistance towards taxanes and vinca-alkaloids and is a common feature of human cancer including NSCLC [99]. The miR-451 is a direct regulator of the multidrug resistant protein (MDR-1). Overexpression of miR-451 induces chemosensitivity while miR-451 loss induces taxane resistance in NSCLC. NOTCH1, through the activation of AP-1, an early transcription factor necessary for progression through G1 phase, downregulates miR451. NOTCH blockade using $\gamma$-secretase inhibitors (GSI) increases miR-451 and reduces MDR-1 thereby sensitizing tumors to taxane-based treatment [100]. In docetaxel-resistant lung cancer cell lines, miR-451 is downregulated which causes Myc/ERK-dependent inactivation of glycogen synthase kinase 3 (GSK-3ß), Snail activation, and EMT [101]. Whether the EMT induced docetaxel resistance in these models is reversible by blocking NOTCH is not yet known. In a recent study, a small molecule $\gamma$-secretase inhibitor (BMS-906024) sensitized NSCLC cell lines to paclitaxel and both drugs synergized preclinically by targeting the paclitaxel-induced increase in NOTCH1, especially in cell lines with a KRAS and BRAF wild type background versus their mutant counterparts, in a TP53-dependent manner [102]. 
Factors from immune, stromal, and cancer cells secreted by paclitaxel-resistant lung adenocarcinoma cells contribute to acquired drug resistance by promoting cell proliferation and escaping apoptosis. However, the secretion of some of these cellgrowth promoting factors is reduced when glucose is deprived. FOXO3a promotes cross-resistance (e.g. to 5-fluorouracil and cisplatin) via glycolysis-mediated ABCB1 upregulation. Suppression of the cellular energy supply by targeting glycolysis may alternatively overcome acquired drug resistance [103]. Genes encoding proteins involved in glucose uptake, glycolysis, lactate to pyruvate conversion, and repression of the tricarboxylic acid cycle are direct transcriptional targets of NOTCH signaling. NOTCH upregulation in breast cancer cells leads to increased glycolysis through activation of the PI3K/AKT pathway, whereas endogenous Notch signaling decreases mitochondrial activity and induces glycolysis in a p53-dependent manner [104].

Additionally, NOTCH signaling also cross-talks with HIF-1 $\alpha$, an important glycolysis regulator [105], through physical interaction with NICD-1 under hypoxia, upregulating NOTCH downstream targets (Hesl, Hey2 mRNA) and preventing differentiation in cortical neural stem cells thus maintaining stemness [106, 107]. Hypoxia $\left(\leq 2 \% \mathrm{O}_{2}\right)$ can induce multi-drug resistance (e.g. to cisplatin, carboplatin, paclitaxel, gemcitabine) in NSCLC via upregulation of ABCB1 and EGF-like domain 7, an endothelial secreted factor that regulates vascular tube formation [91]. Microtubule-targeting agents shift the binding of HIF- $1 \alpha$ from actively translating polysomes to inactive ribosomal subunits as for HIF-1 $\alpha$ mRNA translation requires active transport on interphase microtubules [108]. HIF-1 $\alpha$ can also be upregulated and stabilized in an oxygen-independent manner by oncogene signaling through the PI3K/AKT and MAPK/ERK/Ras pathways; both of which are found mutated in human NSCLC. NOTCH1 activates Akt-1 via PTEN repression and induction of the insulin-like growth factor 1 receptor (IGF-1R) in lung adenocarcinoma during hypoxia [109]. The interaction between the NOTCH and hypoxia/HIF pathways thus connects two cancer vulnerabilities. Therefore, changes in the tumor microenvironment that alter energy metabolism or requirements of tumor cells could be exploited as a target for increasing drug sensitivity by targeting the NOTCH pathway. 


\section{Etoposide}

Topoisomerase II enzymes are important in DNA unwinding, strand excision and religation during replication, and cell cycle checkpoint activation after DNA damage. Etoposide is a topoisomerase II inhibitor and induces cell cycle arrest and apoptosis. Etoposide resistance in NSCLC has been partly attributed to NF-KB-mediated ABCB1 drug transporter expression [110]. Upregulation of NF-kB signaling, through loss of TP53 and KRAS mutations, is found in different cancers including lung adenocarcinoma [111]. NF-KB has been shown to function downstream of $\mathrm{NOTCH}$ and facilitates NOTCH target gene expression and tumor formation in pancreatic and T-ALL models [112-114]. Conversely, in breast cancer stem cells, NF-kB upregulates JAGGED1 expression and activates NOTCH signaling [115]. Also, in glioblastoma multiforme, NF-kB/STAT3 signaling pathway regulates activation of the NOTCH pathway [116]. Etoposide resistance may occur via the 5'tyrosyl DNA phosphodiesterase (TDP2), a transcriptional target of mutant TP53 that repairs topoisomerase-mediated DNA damage [117]. As opposed to normal lung patient tissue, $58.5 \%$ of cancer tissues that stained positive for TP53 were also positive for TDP2 [118]. Although no direct link between TDP2 and NOTCH has yet been found in NSCLC, NUMB might have a role based on its involvement in TP53 degradation [96].

\section{* Pemetrexed}

Pemetrexed inhibits thymidylate synthase, dihydrofolate reductase, and glycinamide ribonucleotide formyltransferase, enzymes involved in folate metabolism, purine and pyrimidine synthesis necessary for DNA and RNA synthesis. Pemetrexed and cisplatin are often administered concomitantly and have good overall survival outcomes in non-squamous NSCLC as first-line therapy [22, 119] but treatment resistance is common. Pemetrexed treatment induces replicative stress in the form of single strand breaks, that if not repaired, will lead to the formation of double strand breaks. Cisplatin on the other hand, induces mainly intrastrand DNA crosslinks which need nucleotide excision repair pathways to be repaired dependent on the availability of a great number of nucleotides. Pre-treatment with pemetrexed with sequential cisplatin administration results in additive/synergistic effects in NSCLC cells $[120,121]$. Interestingly, the surviving clones to sequential pemetrexedcisplatin treatment frequently undergo EMT conversion and are enriched for cancer stem cells (CD133, CD44, NANOG, and Oct $4 B$ mRNA upregulation). Blocking 
EMT with a natural flavonoid, kaempferol, overcomes resistance to anti-folate therapy in NSCLC [122]. Based on its role in EMT induction and cancer stem cell maintenance, targeting NOTCH signaling in NSCLC might be an interesting option to overcome pemetrexed-resistance.

\section{Gemcitabine}

Gemcitabine is an anti-metabolite analog of deoxycytidine that is incorporated into DNA and blocks DNA replication. Gemcitabine treatment in lung cancer cell lines induces an increase in Beclin-1 mediated autophagy activation [123]. Inhibition of autophagosome formation using 3-methyladenine, an inhibitor of PI3K, in gemcitabine-resistant lung cancer cells, increased the expression of apoptotic mediators [123]. NOTCH3 is upregulated in patients with gemcitabine-resistance and its knock-down reduces autophagy (LC3-II expression), colony and sphere forming ability in lung cancer cell lines [90]. Since NOTCH signaling also regulates PI3K/Akt signaling, NOTCH inhibition could lead to similar effects as those obtained with 3-methyladenine. Both in lung and pancreas tumor models, NOTCH inhibition with GSI or monoclonal antibodies (mAbs) targeting NOTCH2/3 (tarextumab) sensitizes tumors to gemcitabine [124, 125].

\section{NOTCH-related resistance to Targeted therapies}

\section{Epidermal Growth factor receptor inhibitors}

EGFR mutations occur in $\sim 10-25 \%$ of lung adenocarcinoma patients and these patients benefit, at least initially, to neutralizing treatment with monoclonal antibodies (e.g. cetuximab, necitumumab) and from TKI (e.g. erlotinib, gefitinib, afatinib) [16]. Often, resistance to TKI occurs via mutation of EGFR (e.g. T790M or S492R), oncogenic shift (MET amplification, HER2 upregulation, KRAS activation), impairment of apoptosis (e.g. BH3 domain deletions of BIM) and EMT conversion [126]. Some patients could regain sensitivity to TKI after discontinuing TKI treatment [127]. The EGFR T790M mutation occurs in $62 \%$ of the patients with acquired resistance and has fueled the development of $2^{\text {nd }}$ and $3^{\text {rd }}$ generation TKI (e.g. Osimertinib/ AZD9291) which was FDA-approved for EGFR T790M positive NSCLC patients [128]. However, resistance against AZD9291 has already been described via an alternative mutation: C797S EGFR [129]. 
Erlotinib treatment of EGFR mutated and wildtype NSCLC enriches ALDH expressing $(\mathrm{ALDH}+)$ stem-like cells in a NOTCH3-dependent manner, increases cell death of ALDH- cells, and increases pulmosphere-forming potential [130]. Concomitant treatment with GSI and erlotinib reduces the ALDH+ subpopulation of cells in EGFR-mutated cell lines [130]. Additionally, expression of NOTCH1 and HES1 were found to be upregulated in gefitinib-resistant lung cancer, which could be reversed by NOTCH inhibition resulting in increased apoptosis [131]. Moreover, dual targeting of EGFR and NOTCH2/3 with the CT16 antibody reduces EGFRTKI-induced ALDH+ and RT-induced CD133+ stem cell subpopulations, the EGFR/RT-induced EMT gene signature, and expression of DNA repair genes. Combination of CT16 with RT prevents tumor regrowth of mouse xenografts. However, CT16 was not effective in treating cetuximab-/erlotinib-resistant cell lines [132]. Other studies have shown that there is a differential response to GSI-treatment depending on the EGFR status. NSCLC cell lines with undetectable EGFR protein levels are more sensitive to GSI-treatment since both autophagic and apoptotic machineries are activated [133]. EGFR T790M TKI-resistant NSCLC frequently have high insulin-like growth factor 1 receptor (IGF-1R) expression levels. EGFR heterodimerizes with IGF-1R preventing gefitinib-induced apoptosis [134]. In cells overexpressing IGF-1R, combination treatment of IGF-1R inhibitors and EGFR TKIs (e.g. linsitinib and gefitinib respectively) inhibits proliferation, increases apoptosis, and attenuates VEGF production in NSCLC cells [135]. Additionally, VEGF expression was found to be upregulated in EGFR-mutated lung adenocarcinomas which additionally increase cell survival via activation of Akt and STAT5 pathways [136]. NOTCH1 is known to upregulate IGF-1R and its inhibition sensitizes cells to GSI-induced cell death under hypoxic conditions [109]. VEGF upregulation could potentially be targeted through NOTCH blockade induced IGF$1 \mathrm{R}$ inhibition.

NOTCH activation stimulates endothelial-to-mesenchymal transition (EndMT) by downregulating endothelial markers (vascular endothelial cadherin, platelet endothelial cell adhesion molecule-1, endothelial NO synthase, Tie1/2), upregulating mesenchymal markers ( $\alpha$-smooth muscle actin, fibronectin, platelet-derived growth factor receptors) and migration toward platelet-derived growth factor-BB [137, 138]. EndMT increases the production of cancer-associated fibroblasts (CAF), known to contribute to tumor progression and treatment resistance [139, 140]. Podoplaninexpressing CAF have shown to be implicated in the primary resistance in NSCLC to EGFR TKI [141]. 


\section{Anaplastic Lymphoma Kinase (ALK) inhibitors}

ALK is a tyrosine kinase receptor which belongs to the insulin receptor family. The ALK receptor can undergo a variety of rearrangements which have been estimated to occur in 3-7\% of NSCLC patients. Currently, there are three targeted agents approved for clinical use: crizotinib, ceritinib and alectanib. Phase I trials with crizotinib led to promising results, however most patients develop resistance to crizotinib within 12 months due to de novo ALK mutations (C1156Y, L1196M, G1269A, L1152R), ALK gene amplification or alternative mechanisms such as epithelial-to-mesenchymal transition (EMT) or upregulation of P-glycoprotein [142]. The next-generation ALK inhibitor Alectinib (CH5424802) has shown efficacy in NSCLC [143]. Hypoxia was found to induce resistance to ALK inhibitors crizotinib and alectanib in lung adenocarcinoma by inducing an EMT phenotype [144]. Resistance to ALK inhibitors in NSCLC is mediated by mechanisms previously described to be associated with NOTCH signaling.

\section{Angiogenesis inhibitors}

Anti-angiogenic therapy aims at normalizing vasculature in tumors to improve blood flow and drug delivery. This can be done either by targeting vascular endothelial growth factor (VEGF) (e.g. bevacizumab or ramucirumab), by targeting the DLL4 NOTCH ligand (e.g. enoticumab, demcizumab or MEDI0639) or simultaneously (e.g. HD105). Monotherapy regimens with anti-VEGF inhibitors results in approximately $70 \%$ reduction in vasculature density and arrests blood flow [145]. The surviving fraction of endothelial cells are characterized by reduced VEGFR2 and 3 reversible expression, and interstitial fluid pressure due to vasculature normalization, enabling better drug delivery to the tumor [146]. Moreover, these cells show intrinsic and/or acquired resistance due to upregulation of alternative proangiogenic signals (e.g. FGF, PDGF and TNF- $\alpha$ ) and increase in local hypoxia [147, 148]. DLL4 NOTCH ligand is partially dependent on VEGF signaling for its expression in lung tumor vessels [149]. Blockade of DLL4 in glioma and breast tumors delays tumor growth even in those tumors that are resistant to anti-VEGF therapy [149]. Dual targeting of DLL4 and VEGF using the bispecific antibody HD105 inhibits tumor progression of lung adenocarcinomas and gastric cancers [150]. Studies with mouse tumor cells however, have shown that overexpression of endothelial specific-DLL4/NOTCH signaling in Lewis lung carcinoma xenografts reduces primary tumor growth by reducing VEGF-induced endothelial proliferation, 
tumor vessel density and overall tumor blood supply. On the other hand, tumor vascular maturation and functionality was improved and thus drug delivery was enhanced and metastasis suppressed [151].

\section{KRAS driven tumors}

KRAS mutation is the most frequent mutation (25\%) in non-squamous NSCLC but no targeted therapies are available for clinical use. Several strategies are being tested in clinical trials including MEK inhibitors, focal adhesion kinase inhibitors, cyclindependent kinase inhibitors, and heat shock protein 90 inhibitors [152]. In multicentric trials, Selumetinib, an inhibitor of MEK1/MEK2 downstream of KRAS, showed no improved progression-free survival (PFS) despite extensive preclinical evidence [153]. A retrospective study suggested that patients with KRAS ${ }^{\mathrm{G} 12 \mathrm{C}}$ tumors, prone to activate the RAS-like pathway, display shorter PFS in response to pemetrexed, while patients with KRAS ${ }^{\mathrm{G} 12 \mathrm{D}}$ tumors, prone to activate the PI3K pathway, show short PFS in response to gemcitabine [154]. Given that NOTCH signaling is involved in the activation of both the RAS and PI3K signaling pathways, therapies targeting NOTCH in KRAS-driven tumors could be a promising strategy. In in vitro and in in vivo preclinical NSCLC models, GSI can increase paclitaxel sensitivity particularly in KRAS-wildtype NSCLC suggesting that KRAS/BRAF mutation status may predict combined efficacy of GSI with paclitaxel [102]. Moreover, other studies have shown that GSI can suppress KRAS-driven NSCLC partly by suppressing ERK/MEK signaling by activating ERK phosphatase DUSP [155].

\section{NOTCH Targeting in NSCLC}

There are several approaches to block NOTCH signaling which include $\gamma$-secretase inhibitors (GSI), monoclonal antibodies (mAbs), blocking peptides, and natural compounds. GSIs prevent NOTCH receptor activation by blocking the rate-limiting step in NOTCH activation, the intramembranous cleavage by presenilin containing the $\gamma$-secretase enzyme. Monoclonal antibodies against the negative regulatory region (NRR) of NOTCH, the ligand-binding EGF repeats in NOTCH extracellular domain, or the NOTCH ligand DLL4 in endothelial cells, block NOTCH signaling at several steps in the signaling cascade. Synthetic, stabilized, cell-permeable blocking peptides have been designed mainly to interfere with the formation of NOTCH-CSL-MAML activation complex. Natural, non-toxic compounds have 
gained interest since they have been shown to be associated with decreased cancerrisk in lung cancer [156]. Moreover, certain non-toxic agents including deltatocotrienol (in blueberries), curcumin (in curcuma longa used as flavoring agent), and anthocyanidins (in berries) have shown anti-NOTCH signaling effects in NSCLC.

\section{Preclinical effects of NOTCH inhibitors}

In preclinical studies, NOTCH signaling has been blocked pharmacologically by 1) Gamma-secretase inhibitors (GSI): DAPT, MRK-003, PF-3084014, RO4929079, BMS-708163, LY-685,458, LY-411575 and GSI XX (Table 1), 2) monoclonal antibodies: CT16 (anti-EGFR and anti-NOTCH 2/3), Tarextumab (anti-NOTCH 2/3), HD105 (anti-DLL4 and anti-VEGF), Demcizumab (anti-DLL4), and 3) naturally-occurring NOTCH signaling inhibitors: Nobiletin, Delta-tocotrienol, Curcumin, and Delphinidin (Table 2). Monotherapy usually doesn't render significant responses in terms of reduced proliferation, induction of apoptosis or tumor growth delay, but NOTCH inhibition does enhance the effect of diverse chemotherapeutic agents.

Despite being one of the least potent GSI in preclinical studies [157], DAPT has proven to be efficacious as single agent in treating NSCLC with altered NOTCH signaling pathway members [76], in inducing apoptosis and autophagy, preferentially in cells expressing EGFR wild type or without EGFR expression [133] by inducing cell cycle arrest in G1 and G2/M phases [100], and reducing the ALDH+ stem cell population [158]. Additionally, DAPT can enhance the effects of cisplatin by reducing the CD133+ stem cell subpopulation [159]. DAPT also prevents crossresistance to paclitaxel and doxorubicin [89], of docetaxel by decreasing the AP1/miR-451-induced MDR-1 expression [100], of gefitinib by reverting the EMT phenotype [131, 160], and of pterostilbene by preventing PI3K/Akt activation [161]. Several other GSI have shown to be able to attenuate EGFR TKI resistance to erlotinib: MRK-003 via increase of the ERK-regulated pro-apoptotic Bim [162], PF3084014 through downregulation of ALDH+ stem cells [130], and RO4929079 by decreasing CD44+ stem cells and increasing the miR-223-induced decrease in FBXW7 expression [163]. Additionally, BMS-708163 can revert resistance to gefitinib in NSCLC [164]. MRK-003 has shown therapeutic enhancement of docetaxel [54] and IGF-1R inhibition by inducing apoptosis [109]. GSI-XX enhances therapeutic efficacy of the combination including a HIF-targeting small molecule inhibitor (YC-1) and radiotherapy, but only when added after the combination [165]. GSI-XX also improves the response of cisplatin by promoting autophagy, reducing 
the ALDHA1+ and CD44+ stem cells [90], or when combined with a BH3-mimetic (ABT-737) by inducing apoptosis in a Bim-dependent manner [166]. The doses used in these studies have been summarized in Table 3 .

Monoclonal antibodies against DLL4 used to target the tumor vasculature, result in an increase of branched vessels but with decreased functionality. Combination therapy with bevacizumab increases apoptosis, and decreases vessel branching and tumor progression [150]. One of the major problems with anti-VEGF therapy in the clinic is the increased levels of hypoxia leading to more aggressive treatment resistant tumor cell populations, but it also leads to hemoptysis, hypertension, and arterial thrombus embolism due to effects on the normal vasculature in the heart, and endocrine and nervous systems $[167,168]$. Because these anti-angiogenic inhibitors are not tumor specific, successful clinical implementation will most likely consist of the use of drug doses/scheduling aiming at vessel normalization, promoting formation of functional vessels to improve drug delivery [169], or in their combination with other therapies, rather than those aiming at tumor regression. Tarextumab in combination with erlotinib, cetuximab, or CT16, increases therapeutic efficiency of EGFR targeting and delays acquired resistance by similar mechanisms as those with TKI therapy [132].

Natural-occurring compounds have shown remarkable anti-NOTCH effects not only by affecting the expression of NOTCH pathway members (NOTCH1 and HES1) which results in reversal of the hypoxic-induced EMT phenotype [170], but also by upregulation of miR-34a, TP53 and apoptosis [171]; inhibition of EZH2 [172]; and have shown to be able to potentiate anti-tumorigenic effects in combination with cisplatin [171].

As described in this review, the NOTCH signaling pathway acts upon different aspects of the hallmarks of cancer described by Hanahan and Weinberg to promote cancer resistance, and its targeting can enhance the effect of chemotherapeutics or agents targeting oncogenic driver mutations when used in combination (see Figure 1 for summary). Importantly, several studies have shown that NOTCH inhibition in combination with radiation therapy also improves outcome in NSCLC [165, 173, 174]. Since most patients receive combinations of radiotherapy and chemotherapy, these results are significant and show there is great potential for combining NOTCH inhibitors with radiotherapy and chemotherapy to target the cancer stem cells and reduce treatment resistance. Further analysis on the implications of combining NOTCH-based targeted therapy with radiotherapy have been recently described [78, 175]. 


\section{Clinical trials using NOTCH pathway inhibitors}

Only a few of the preclinically tested NOTCH inhibitors have progressed into clinical trials in NSCLC patients including BMS-906024, PF-3084014, RO4929079, MK0752, LY900009, LY3039478, BMS-986115, enoticumab, demcizumab, and MEDI0639 (Table 4 and 5). Clinical studies using Notch inhibitors as monotherapy have shown limited effect on local control and some have been halted [176, 177]. Nevertheless, conclusions on GSI effects in cancer patients are debatable since patients are not pre-screened for Notch signaling upregulation/mutation and different clinical GSIs have diverse potencies, specificities, and side effects [157].

In the clinical trials including lung cancer patients, the best responses using GSI were obtained with LY900009 as single agent (NCT-01158404) with which 5/35 stable diseases were accomplished. GSI efficacy was confirmed by measuring on-target undesirable effects of NOTCH inhibition. Low clinical activity was explained by fast drug absorption and elimination [178]. Combination of RO4929097 with cediranib or temsirolimus (PJC-004/ NCI 8503) only marginally improved outcome, obtaining at best one partial response and 11/17-19 stable diseases with a median progressionfree survival of 4.2 months $[179,180]$. In the latter studies, there was no association between NOTCH biomarkers and time to progression at the recommended phase II dose.

Monoclonal antibodies against DLL4 have shown better outcomes compared to GSI treatment. DLL4 has been shown to be both important for tumor vasculature as well as for maintaining tumor initiating capacity of tumor stem cells in various tumor models [181]. As single agents (NCT-01577745), enoticumab, demcizumab and MEDI0639 may have up to two partial responses (enoticumab, MEDI0639) and between 36-44\% of stable diseases lasting for an average of 6 months [182, 183]. Blood of demcizumab-treated patients presented a decrease in NOTCH and WNT pathway members, and an increase of their negative regulators, whereas the hair follicles had a downregulation of stem cell genes, and an upregulation of vascular genes. In enoticumab-treated patients however, there was no association between $\mathrm{NOTCH}$ biomarkers and time to disease progression. Clinical trials combining demcizumab with pemetrexed and carboplatin for non-squamous NSCLC (NCT01189968) obtained objective responses in 50\% of patients, demcizumabtarget efficacy was observed in the blood, and a demcizumab dose was recommended for phase II trials [184]. Phase II trials (YOSEMITE) of demcizumab in combination with paclitaxel and gemcitabine to treat metastatic pancreatic cancer however were recently discontinued because primary efficacy endpoints (progression-free survival) 
were not significantly better than placebo (chemotherapy alone) however, results on other trials (PINNACLE, DENALI) are yet to be evaluated. It remains to be evaluated how combination therapy with demcizumab would influence survival in NSCLC patients.

\section{Side-effects of NOTCH Therapeutics}

Gastrointestinal toxicity is an undesired on-target effect of $\gamma$-secretase inhibitors due to simultaneous inhibition of NOTCH1 and 2, which have redundant roles in regulating homeostasis in the crypt epithelium, resulting in excessive secretory differentiation and goblet cell metaplasia [185-189]. GSIs used preclinically (Tables 3 and 6) result mainly in gastrointestinal toxicity, as seen for MRK-003 [190], PF3084014 [191], LY-411575 [188, 192], DBZ [187, 189, 193] and compound E [194]. These effects are usually found in combination with severe body weight loss but can be mitigated using glucocorticoids (dexamethasone) as shown for PF-3084014 [191] and DBZ [195]. The use of monoclonal antibodies such as OMP59R5 minimizes associated intestinal toxicity [124] probably because NOTCH1, one of the major contributors of normal gastrointestinal architecture, is not targeted. No gastrointestinal effects were detected for anti-DLL4 antibodies [196], which is explained by redundancy with DLL1 [197], and natural agents, except for curcumin where loose bowel movements were reported [198]. In clinical trials (Tables 4 and 7), several studies reported severe gastrointestinal toxicities (grade III or higher) including diarrhea, nausea, dehydration and mucositis for RO4929097 [179, 180], LY900009 [178] and MK-0752 [199] (Table 7). However, these effects were mitigated with adequate scheduling [200]. Oral dosing of PF-03084014 twice a day resulted in manageable gastrointestinal toxicity, better than that for RO4929097 and MK0752 [201], indicative that not all GSI are equally potent nor biological equivalents because they target different NOTCH receptors and also have diverse off-target effects [157]. In clinical trials (NCT-01577745), only abdominal pain was reported for enoticumab [182], and gastrointestinal toxicity was milder than for GSI treatment in general [183].

Skin adverse effects occur after GSI treatment due to the physiological function of Notch signaling in keratinocyte differentiation [192]. In preclinical studies (Table 6), $\mathrm{NOTCH}$ inhibition may cause epidermal and epithelial cell hyperplasia, and follicular and epidermal inclusion cysts $[188,192]$. Natural inhibitors of NOTCH signaling showed mild to moderate dermatitis at the site of injection for deltatocotrienol [202] and piloerection for high doses of curcumin [198]. In patients 
(Table 7), skin toxicity (grade III or higher) has been reported for GSI (but not for anti-DLL4 antibodies) and is attributed exclusively to skin rash for RO4929097 [179, 180] and MK-0752 [199].

In the immune system, NOTCH regulates megakaryocyte development and the myeloid and erythroid lineages [203]. In preclinical studies (Table 6), NOTCH inhibition may lead to mild eosinophilic inflammation, leukopenia, lymphocytopenia, altered lymphocyte development, thymus atrophy, and/or splenic marginal zone lymphoid tissue atrophy for PF-3084014 [191], LY-411575 [188, 192] and DBZ [187]. In patients (Table 7), no severe toxicities (grade III or higher) were reported.

In the vasculature, there are also toxicities reported in preclinical studies (Table 6), accounting for pathological activation of endothelial cells and sinusoidal dilation for HD105 [204]. In patients (Table 7), there were toxicities (grade III or higher) reported accounting for cardiac arrest, tachycardia, ventricular dysfunction, congestive heart failure for the GSI RO4929097 (NCT-0119386; NCT-01217411), for enoticumab [182] and for demcizumab [183].

In the lung, there were generally no effects reported preclinically (Table 6) except for respiratory distress at very high doses of curcumin [198]. In patients (Table 7) however, toxicities (grade III or higher) included lung infection/sepsis, dyspnea, and pulmonary hypertension for RO4929097 (NCT-0119386; NCT-01217411) and for enoticumab [182].

\section{NOTCH inhibition in Lung cancer Comorbidities}

Lung cancer patients often present several comorbidities. When considering the potential for $\mathrm{NOTCH} / \gamma$-secretase inhibitors, normal tissue effects are usually doselimiting. The potential role of NOTCH inhibition has been investigated not only in lung cancer but also in other lung pathologies, such as pulmonary goblet cell metaplasia, lung fibrosis, allergic asthma, chronic inflammation, and pulmonary arterial hypertension. The pathophysiology of pulmonary goblet cell metaplasia is similar to that of cystic fibrosis, bronchitis, and asthma. NOTCH inhibition may be of potential benefit in such cases by reducing the number of secretory cells and altering basal cell differentiation in adult lung towards a ciliated phenotype [205]. In lung fibrosis, characterized by fibroblast proliferation leading to excess extracellular matrix deposition (collagen and glycosaminoglycans) and tissue remodeling 
occurring frequently 6-9 months after radiation treatment, GSI prevents NOTCH/JAGGED1-induced myofibroblast differentiation in response to FZZ1 by decreasing the expression of $\alpha$-SMA $[138,206]$. Asthma and chronic inflammation (which may occur after radiation treatment) are characterized by airway hyperresponsiveness and enhanced immune response. NOTCH receptors are expressed in the surface of mature lymphocytes whereas NOTCH ligands are present in antigen-presenting cells. Inhibition of airway hyperresponsiveness and inflammation by repressing Th2-mediated IL-4 production can be observed with the inhibition of NOTCH on CD4+ T-cells and JAGGED1 on bone marrow dendritic cells [207]. Pulmonary veno-oclusive disease (PVOD), an uncommon cause of pulmonary arterial hypertension (PAH), may appear after combined therapy consisting of surgery and mitomycin with perioperative chemotherapy. PVOD requires upregulation of NOTCH3, smooth muscle cell proliferation in small pulmonary arteries, and increased vascular resistance to develop. PAH severity correlates with NOTCH3 and its downstream effector HES5. Thus, targeting the NOTCH3-HES5 axis with GSIs may improve PAH treatment [208].

\section{Conclusions and Perspectives}

Non-squamous NSCLC is the most common form of lung cancer and a deadly disease. Despite detailed knowledge on tumor driver mutations and multimodal treatment regimens with surgery, and systemic treatment using chemotherapeutics, targeted agents, and radiotherapy, tumor resistance, relapse and dose limiting toxicities are common. NSCLC is a highly heterogeneous disease at different levels and is constantly striving for survival by acquiring new favorable pro-survival mutations. There is mounting evidence implicating cancer stem cells, and the dynamically changing tumor microenvironment, as the drivers of tumor heterogeneity which in turn results in tumor progression, metastasis, and therapeutic resistance. Cancer stem cells provide an interesting therapeutic targeting opportunity to tackle tumor resistance. There is paramount evidence for a role of the NOTCH signaling pathway in driving tumor-resistance through cancer (stem) cells and crosstalk with other pathways. We identified roles for NOTCH signaling in mechanisms of tumor resistance mediated via, mainly, $\mathrm{ABC}$ drug transporters, epithelialmesenchymal transition, hypoxia, pro-survival pathways and VEGF, among others (Figure 1).

Monotherapy treatment using NOTCH inhibitors, similarly to what happens for other therapies, is not sufficient to induce tumor control or cure. Because not all patients 
have an aberrantly high NOTCH expression, and NOTCH-based therapeutics targets a specific subpopulation which is a minority in the bulk of the tumor, it should not be expected that monotherapy NOTCH-targeting treatments will have a significant impact on clinical outcome. Unfortunately, many clinical trials using NOTCH therapeutics are terminated, on hold or have reported limited efficacy. Parallel to this observation, it must be noted that the current interventional setting to evaluate NOTCH inhibition efficiency is sub-optimal. The most important shortcoming of the clinical trials is the lack of robust biomarkers to select patients and monitor responses to treatment. While there are many gene expression studies investigating on and off target effects of NOTCH targeting using GSI, they reflect the combined effects of NOTCH1-4 receptor blockade. Since Notch signaling in lung tissue is complex, with redundant but also opposing functions of specific NOTCH isoforms affecting prognosis and treatment sensitivity, such signatures are unlikely to yield predictive markers for patient stratification. It is anticipated that selection of patients would improve the quality and accuracy of the information from clinical trials. Companion biomarkers that are assessed dynamically over the course of cancer treatment are needed to monitor on-target efficacy and therapeutic redesigning.

NOTCH receptor or ligand-specific targeting agents, as opposed to broad spectrum small molecule $\gamma$-secretase inhibitors, are more likely to induce less adverse effects however, proper scheduling, reduced dosing, and combination with glucocorticoids can mitigate the adverse effects of GSIs. New insights into $\gamma$-secretase's complex composition may yield receptor-specific small molecule NOTCH inhibitors [209] that could aid in attenuating the side effects of broad spectrum inhibitors. Monoclonal antibodies have minimal toxicity to the gastrointestinal tract but they generally have limited biodistribution and prolonged half-life, a shortcoming that can be addressed using $\mathrm{F}\left(\mathrm{ab}^{\prime}\right)_{2}$ antibody fragments which allow more flexible control over the extent and duration of inhibition. Soluble decoys of the extracellular domain of NOTCH or its ligands block receptor-ligand interaction, however, their efficacy depends on their biodistribution and pharmacokinetic properties which remain to be assessed. On the other hand, cancers may present more than one alteration that increases the activity of the NOTCH signaling pathway, therefore, the benefits of pan-NOTCH inhibitors, which target all four NOTCH receptors, versus selective NOTCH receptor targeting needs further examination.

We discussed how NOTCH pathway inhibition can enhance chemo-/targeted agent sensitivity and even revert resistance in NSCLC. Although out of the scope of this review, similar opportunities exist for combining NOTCH inhibition with radiation 
therapy [174], and potentially, immune therapies. Because NOTCH signaling impacts several features of the tumor microenvironment such as tumor hypoxia and tumor cell metabolism, the prospect of altering the microenvironment using NOTCH inhibitors is exciting since normal cells usually do not develop drug resistance, but challenging because of the adverse effects of NOTCH inhibitors on normal tissue. Recently, there has been a report that T-ALL cells restore NOTCH signaling activation upon GSI withdrawal, suggesting that the persisting cells are reversibly resistant to GSI through epigenetic alterations. Combination therapies with epigenetic modulators however, enhance NOTCH-inhibition therapy [210]. Nevertheless, it remains to be established whether epigenetic modifiers can prolong GSI effects in lung cancer with tolerable normal tissue effects.

In summary, lung cancer is a complex heterogenous disease with interpatient, intratumor and inter-/intrametastatic heterogeneity at the subtype level, therefore, successful treatment options are likely to arise from personalized precision treatment. There is paramount preclinical evidence for potent anti-tumor activity of NOTCH therapeutics in NSCLC but our biological understanding of the tissue and contextspecific roles of NOTCH is understudied. Biomarkers will be essential to advance into clinical development to obtain meaningful and reliable answers on therapeutic ratios. Finally, while there is much attention into the development of smarter drugs to target specific drivers of progression and treatment resistance, efforts should also be directed towards identifying synergistic interactions of Notch inhibitors with clinically approved systemic treatments as such combinations are likely to lead to faster clinical implementation and hence, benefit the patients.

\section{Literature and Search criteria}

Studies including chemotherapeutics or targeted agents used as part of standard of care treatment against NSCLC, studies that included NOTCH signaling, nonsquamous NSCLC, preclinical and clinical studies, and studies using GSI, monoclonal antibodies, or natural agents to target NOTCH pathway members. Whenever possible, we analyzed data from studies after the year 2000. 


\section{6 | Chapter 2}

\section{Acknowledgements}

This work was supported by World-Wide Cancer research (AICR 1311-97), ERCCoG, (DIRECT 617060), and Marie Curie International Training Network (ITN) grants (Radiate, 642623). 


\section{References}

1. Auperin, A., et al., Meta-analysis of concomitant versus sequential radiochemotherapy in locally advanced non-small-cell lung cancer. J Clin Oncol, 2010. 28(13): p. 2181-90.

2. Huber, R.M., et al., Simultaneous chemoradiotherapy compared with radiotherapy alone after induction chemotherapy in inoperable stage IIIA or IIIB non-small-cell lung cancer: study CTRT99/97 by the Bronchial Carcinoma Therapy Group. J Clin Oncol, 2006. 24(27): p. 4397-404.

3. Ceresoli, G.L., et al., Phase II study of weekly paclitaxel as second-line therapy in patients with advanced non-small cell lung cancer. Lung Cancer, 2004. 44(2): p. 231-9.

4. Weiss, G.J., et al., The impact of induction chemotherapy on the outcome of second-line therapy with pemetrexed or docetaxel in patients with advanced non-small-cell lung cancer. Ann Oncol, 2007. 18(3): p. 453-60.

5. Veronesi, A., et al., Chemotherapy of advanced non-small-cell lung cancer with cyclophosphamide, adriamycin, methotrexate, and procarbazine versus cisplatin and etoposide. A randomized study. Am J Clin Oncol, 1988. 11(5): p. 566-71.

6. Yamazaki, S., et al., Clinical responses of large cell neuroendocrine carcinoma of the lung to cisplatin-based chemotherapy. Lung Cancer, 2005. 49(2): p. 217-23.

7. Sun, J.-M., et al., Chemotherapy for pulmonary large cell neuroendocrine carcinoma: Similar to that for small cell lung cancer or non-small cell lung cancer? Lung Cancer, 2012. 77(2): p. 365-370.

8. Iyoda, A., et al., Treatment options for patients with large cell neuroendocrine carcinoma of the lung. General Thoracic and Cardiovascular Surgery, 2014. 62(6): p. 351-356.

9. Noronha, V., et al., EGFR mutations in Indian lung cancer patients: clinical correlation and outcome to EGFR targeted therapy. PLoS One, 2013. 8(4): p. e61561.

10. Joshi, A., et al., EGFR mutation in squamous cell carcinoma of the lung: does it carry the same connotation as in adenocarcinomas? Onco Targets Ther, 2017. 10: p. 1859-1863.

11. Shukuya, T., et al., Efficacy of gefitinib for non-adenocarcinoma non-smallcell lung cancer patients harboring epidermal growth factor receptor mutations: a pooled analysis of published reports. Cancer Sci, 2011. 102(5): p. 1032-7.

12. Miller, V.A., et al., Bronchioloalveolar pathologic subtype and smoking history predict sensitivity to gefitinib in advanced non-small-cell lung cancer. J Clin Oncol, 2004. 22(6): p. 1103-9.

13. Imielinski, M., et al., Mapping the hallmarks of lung adenocarcinoma with massively parallel sequencing. Cell, 2012. 150(6): p. 1107-20. 
14. Govindan, R., et al., Genomic landscape of non-small cell lung cancer in smokers and never-smokers. Cell, 2012. 150(6): p. 1121-34.

15. Ding, L., et al., Somatic mutations affect key pathways in lung adenocarcinoma. Nature, 2008. 455(7216): p. 1069-75.

16. Lin, J.J. and A.T. Shaw, Resisting Resistance: Targeted Therapies in Lung Cancer. Trends Cancer, 2016. 2(7): p. 350-364.

17. Dagogo-Jack, I., A.T. Shaw, and G.J. Riely, Optimizing treatment for patients with anaplastic lymphoma kinase-positive lung cancer. Clin Pharmacol Ther, 2017. 101(5): p. 625-633.

18. Thomas, A., et al., Refining the treatment of NSCLC according to histological and molecular subtypes. Nat Rev Clin Oncol, 2015. 12(9): p. 511-26.

19. Joshi, A., et al., EGFR mutation in squamous cell carcinoma of the lung: does it carry the same connotation as in adenocarcinomas? OncoTargets and therapy, 2017. 10: p. 1859-1863.

20. Cancer Genome Atlas Research, N., Comprehensive genomic characterization of squamous cell lung cancers. Nature, 2012. 489(7417): $\mathrm{p}$. 519-25.

21. Herbst, R.S., Toxicities of Antiangiogenic Therapy in Non-Small-Cell Lung Cancer. Clinical Lung Cancer, 2006. 8: p. S23-S30.

22. Besse, B., et al., 2nd ESMO Consensus Conference on Lung Cancer: nonsmall-cell lung cancer first-line/second and further lines of treatment in advanced disease. Ann Oncol, 2014. 25(8): p. 1475-84.

23. Hellmann, M.D., et al., Nivolumab plus ipilimumab as first-line treatment for advanced non-small-cell lung cancer (CheckMate 012): results of an open-label, phase 1, multicohort study. Lancet Oncol, 2017. 18(1): p. 31-41.

24. Antonia, S.J., et al., Durvalumab after Chemoradiotherapy in Stage III NonSmall-Cell Lung Cancer. N Engl J Med, 2017. 377(20): p. 1919-1929.

25. McCall, N.S., A.P. Dicker, and B. Lu, Beyond Concurrent Chemoradiation: The Emerging Role of PD-1/PD-L1 Inhibitors in Stage III Lung Cancer. Clin Cancer Res, 2018. 24(6): p. 1271-1276.

26. Riaz, N., et al., Tumor and Microenvironment Evolution during Immunotherapy with Nivolumab. Cell, 2017.

27. Somasundaram, A. and T.F. Burns, The next generation of immunotherapy: keeping lung cancer in check. Journal of Hematology \& Oncology, 2017. 10: p. 87.

28. Alexandrov, L.B., et al., Signatures of mutational processes in human cancer. Nature, 2013. 500(7463): p. 415-21.

29. Nagy, A., et al., KRAS driven expression signature has prognostic power superior to mutation status in non-small cell lung cancer. Int J Cancer, 2017. 140(4): p. 930-937.

30. Shukla, S., et al., Development of a RNA-Seq Based Prognostic Signature in Lung Adenocarcinoma. J Natl Cancer Inst, 2017. 109(1). 
31. Vogelstein, B., et al., Cancer genome landscapes. Science, 2013. 339(6127): p. $1546-58$.

32. Gerlinger, M., et al., Intratumor heterogeneity and branched evolution revealed by multiregion sequencing. N Engl J Med, 2012. 366(10): p. 883892.

33. Fidler, I.J., Tumor heterogeneity and the biology of cancer invasion and metastasis. Cancer Res, 1978. 38(9): p. 2651-60.

34. Gerlinger, M., et al., Cancer: evolution within a lifetime. Annu Rev Genet, 2014. 48: p. 215-36.

35. Hensley, C.T., et al., Metabolic Heterogeneity in Human Lung Tumors. Cell, 2016. 164(4): p. 681-94.

36. Carvalho, S., et al., Prognostic value of metabolic metrics extracted from baseline positron emission tomography images in non-small cell lung cancer. Acta Oncol, 2013. 52(7): p. 1398-404.

37. Weir, B.A., et al., Characterizing the cancer genome in lung adenocarcinoma. Nature, 2007. 450(7171): p. 893-8.

38. Zhang, J., et al., Intratumor heterogeneity in localized lung adenocarcinomas delineated by multiregion sequencing, in Science. 2014. p. 256-259.

39. Rosenbaum, J.N., et al., Genomic heterogeneity of ALK fusion breakpoints in non-small-cell lung cancer. Mod Pathol, 2018.

40. McGranahan, N., et al., Clonal status of actionable driver events and the timing of mutational processes in cancer evolution. Sci Transl Med, 2015. 7(283): p. 283 ra54.

41. Jamal-Hanjani, M., et al., Tracking genomic cancer evolution for precision medicine: the lung TRACERx study. PLoS Biol, 2014. 12(7): p. e1001906.

42. Bonnet, D. and J.E. Dick, Human acute myeloid leukemia is organized as a hierarchy that originates from a primitive hematopoietic cell. Nat Med, 1997. 3(7): p. 730-7.

43. Prasetyanti, P.R. and J.P. Medema, Intra-tumor heterogeneity from a cancer stem cell perspective. Mol Cancer, 2017. 16(1): p. 41.

44. Wang, P., et al., Cancer stem-like cells can be induced through dedifferentiation under hypoxic conditions in glioma, hepatoma and lung cancer. Cell Death Discov, 2017. 3: p. 16105.

45. Carney, D.N., et al., Demonstration of the stem cell nature of clonogenic tumor cells from lung cancer patients. Stem Cells, 1982. 1(3): p. 149-64.

46. Ho, M.M., et al., Side population in human lung cancer cell lines and tumors is enriched with stem-like cancer cells. Cancer Res, 2007. 67(10): p. 482733.

47. Eramo, A., et al., Identification and expansion of the tumorigenic lung cancer stem cell population. Cell Death Differ, 2008. 15(3): p. 504-14.

48. Kaiser, J., The cancer stem cell gamble. Science, 2015. 347(6219): p. 226229. 
49. Takebe, N., et al., Targeting cancer stem cells by inhibiting Wnt, Notch, and Hedgehog pathways. Nature reviews. Clinical oncology, 2010. 8(2): p. 97106.

50. Armstrong, F., et al., NOTCH is a key regulator of human T-cell acute leukemia initiating cell activity. Blood, 2009. 113(8): p. 1730-40.

51. Fan, X., et al., Notch pathway inhibition depletes stem-like cells and blocks engraftment in embryonal brain tumors. Cancer Res, 2006. 66(15): p. 744552.

52. Harrison, H., et al., Breast cancer stem cells: something out of notching? Cancer Res, 2010. 70(22): p. 8973-6.

53. Sikandar, S.S., et al., NOTCH signaling is required for formation and selfrenewal of tumor-initiating cells and for repression of secretory cell differentiation in colon cancer. Cancer Res, 2010. 70(4): p. 1469-78.

54. Hassan, K.A., et al., Notch pathway activity identifies cells with cancer stem cell-like properties and correlates with worse survival in lung adenocarcinoma. Clin Cancer Res, 2013. 19(8): p. 1972-80.

55. Artavanis-Tsakonas, S., M.D. Rand, and R.J. Lake, Notch signaling: cell fate control and signal integration in development. Science, 1999. 284(5415): p. 770-6.

56. Bray, S.J., Notch signalling: a simple pathway becomes complex. Nat Rev Mol Cell Biol, 2006. 7(9): p. 678-89.

57. van Tetering, G. and M. Vooijs, Proteolytic cleavage of Notch: "HIT and $R U N^{\prime \prime}$. Curr Mol Med, 2011. 11(4): p. 255-69.

58. Borggrefe, T. and F. Oswald, The Notch signaling pathway: Transcriptional regulation at Notch target genes. Cellular and Molecular Life Sciences, 2009. 66(10): p. 1631-1646.

59. Ranganathan, P., K.L. Weaver, and A.J. Capobianco, Notch signalling in solid tumours: a little bit of everything but not all the time. Nat Rev Cancer, 2011. 11(5): p. 338-51.

60. Weng, A.P., et al., Activating Mutations of <em>NOTCH1</em > in Human T Cell Acute Lymphoblastic Leukemia. Science, 2004. 306(5694): p. 269271.

61. Hanahan, D. and R.A. Weinberg, Hallmarks of cancer: the next generation. Cell, 2011. 144(5): p. 646-674.

62. Aster, J.C., W.S. Pear, and S.C. Blacklow, The Varied Roles of Notch in Cancer. Annu Rev Pathol, 2017. 12(1): p. 245-275.

63. Kong, Y., et al., Functional diversity of notch family genes in fetal lung development. Am J Physiol Lung Cell Mol Physiol, 2004. 286(5): p. L107583.

64. Tsao, P.N., et al., Gamma-secretase activation of notch signaling regulates the balance of proximal and distal fates in progenitor cells of the developing lung. The Journal of biological chemistry, 2008. 283(43): p. 29532-44. 
65. Tsao, P.-N., et al., Notch signaling controls the balance of ciliated and secretory cell fates in developing airways. Development (Cambridge, England), 2009. 136(13): p. 2297-2604.

66. Morimoto, M., et al., Canonical Notch signaling in the developing lung is required for determination of arterial smooth muscle cells and selection of Clara versus ciliated cell fate. J Cell Sci, 2010. 123(Pt 2): p. 213-24.

67. Rock, J.R., et al., Notch-dependent differentiation of adult airway basal stem cells. Cell Stem Cell, 2011. 8(6): p. 639-48.

68. Lafkas, D., et al., Therapeutic antibodies reveal Notch control of transdifferentiation in the adult lung. Nature, 2015. 528(7580): p. 127-31.

69. Tsao, P.N., et al., Epithelial Notch signaling regulates lung alveolar morphogenesis and airway epithelial integrity. Proc Natl Acad Sci U S A, 2016. 113(29): p. 8242-7.

70. Morimoto, M., et al., Different assemblies of Notch receptors coordinate the distribution of the major bronchial Clara, ciliated and neuroendocrine cells. Development, 2012. 139(23): p. 4365-73.

71. Xing, Y., et al., NOTCH1 is required for regeneration of Clara cells during repair of airway injury. Stem Cells, 2012. 30(5): p. 946-55.

72. Aster, J.C., W.S. Pear, and S.C. Blacklow, The Varied Roles of Notch in Cancer. Annual Review of Pathology: Mechanisms of Disease, 2017. 12(1): p. 245-275.

73. Mutvei, A.P., E. Fredlund, and U. Lendahl, Frequency and distribution of Notch mutations in tumor cell lines. BMC Cancer, 2015. 15: p. 311.

74. Dang, T.P., et al., Chromosome 19 translocation, overexpression of Notch3, and human lung cancer. J Natl Cancer Inst, 2000. 92(16): p. 1355-7.

75. Haruki, N., et al., Dominant-negative Notch3 receptor inhibits mitogenactivated protein kinase pathway and the growth of human lung cancers. Cancer Res, 2005. 65(9): p. 3555-61.

76. Westhoff, B., et al., Alterations of the Notch pathway in lung cancer. Proc Natl Acad Sci U S A, 2009. 106(52): p. 22293-8.

77. Jin, M.M., et al., Notch signaling molecules as prognostic biomarkers for non-small cell lung cancer. Oncol Lett, 2015. 10(5): p. 3252-3260.

78. Theys, J., et al., High NOTCH activity induces radiation resistance in non small cell lung cancer. Radiotherapy and Oncology, 2013. 108(3): p. 440445.

79. Chen, C.Y., et al., Expression of Notch Gene and Its Impact on Survival of Patients with Resectable Non-small Cell Lung Cancer. J Cancer, 2017. 8(7): p. 1292-1300.

80. Xiong, J., et al., Prognostic roles of mRNA expression of notch receptors in non-small cell lung cancer. Oncotarget, 2017. 8(8): p. 13157-13165.

81. Yuan, X., et al., Meta-analysis reveals the correlation of Notch signaling with non-small cell lung cancer progression and prognosis. Sci Rep, 2015. 5: p. 10338. 
82. Zhang, M., et al., Does Notch play a tumor suppressor role across diverse squamous cell carcinomas? Cancer Med, 2016. 5(8): p. 2048-60.

83. Nicolas, M., et al., Notch1 functions as a tumor suppressor in mouse skin. Nat Genet, 2003. 33(3): p. 416-21.

84. Wang, N.J., et al., Loss-of-function mutations in Notch receptors in cutaneous and lung squamous cell carcinoma. Proc Natl Acad Sci U S A, 2011. 108(43): p. 17761-6.

85. Egloff, A.M. and J.R. Grandis, Molecular Pathways: Context-dependent approaches to Notch targeting as cancer therapy. Clinical cancer research : an official journal of the American Association for Cancer Research, 2012. 18(19): p. 5188-5195.

86. Weinstein, I.B., Cancer. Addiction to oncogenes--the Achilles heal of cancer. Science, 2002. 297(5578): p. 63-4.

87. Diaz, L.A., et al., The molecular evolution of acquired resistance to targeted EGFR blockade in colorectal cancers. Nature, 2012: p. 1-4.

88. Gorre, M.E., et al., Clinical resistance to STI-571 cancer therapy caused by BCR-ABL gene mutation or amplification. Science, 2001. 293(5531): p. 87680.

89. Liu, Y.P., et al., Cisplatin selects for multidrug-resistant CD133+ cells in lung adenocarcinoma by activating Notch signaling. Cancer Res, 2013. 73(1): p. 406-16.

90. Ma, Y., et al., Blockade of Notch3 inhibits the stem-like property and is associated with ALDHIA1 and CD44 via autophagy in non-small lung cancer. Int J Oncol, 2016. 48(6): p. 2349-58.

91. Yu, W.K., et al., Chemoresistant lung cancer stem cells display high DNA repair capability to remove cisplatin-induced DNA damage. $\mathrm{Br} \mathrm{J}$ Pharmacol, 2017. 174(4): p. 302-313.

92. Barr, M.P., et al., Generation and Characterisation of Cisplatin-Resistant Non-Small Cell Lung Cancer Cell Lines Displaying a Stem-Like Signature. PLOS ONE, 2013. 8(1): p. e54193.

93. Espinoza, I. and L. Miele, Deadly crosstalk: Notch signaling at the intersection of EMT and cancer stem cells. Cancer Lett, 2013. 341(1): p. 415 .

94. Wang, Z., et al., The role of Notch signaling pathway in epithelialmesenchymal transition (EMT) during development and tumor aggressiveness. Curr Drug Targets, 2010. 11(6): p. 745-51.

95. Rusch, V., et al., Aberrant p53 expression predicts clinical resistance to cisplatin-based chemotherapy in locally advanced non-small cell lung cancer. Cancer Res, 1995. 55(21): p. 5038-42.

96. Colaluca, I.N., et al., NUMB controls p53 tumour suppressor activity. Nature, 2008. 451(7174): p. 76-80.

97. Menietti, E., et al., Negative control of CSL gene transcription by stress/DNA damage response and p53. Cell Cycle, 2016. 15(13): p. 1767-78. 
98. McAuliffe, S.M., et al., Targeting Notch, a key pathway for ovarian cancer stem cells, sensitizes tumors to platinum therapy. Proc Natl Acad Sci U S A, 2012. 109(43): p. E2939-48.

99. Zaman, G.J., et al., Analysis of the expression of MRP, the gene for a new putative transmembrane drug transporter, in human multidrug resistant lung cancer cell lines. Cancer Res, 1993. 53(8): p. 1747-50.

100. Huang, J., et al., Notch-1 Confers Chemoresistance in Lung Adenocarcinoma to Taxanes through AP-1/microRNA-451 Mediated Regulation of MDR-1. Mol Ther Nucleic Acids, 2016. 5: p. e375.

101. Chen, D., et al., MicroRNA-451 induces epithelial-mesenchymal transition in docetaxel-resistant lung adenocarcinoma cells by targeting protooncogene c-Myc. Eur J Cancer, 2014. 50(17): p. 3050-67.

102. Morgan, K.M., et al., Gamma Secretase Inhibition by BMS-906024 Enhances Efficacy of Paclitaxel in Lung Adenocarcinoma. Mol Cancer Ther, 2017. 16(12): p. 2759-2769.

103. Aldonza, M.B.D., J.-Y. Hong, and S.K. Lee, Paclitaxel-resistant cancer cell-derived secretomes elicit ABCB1-associated docetaxel cross-resistance and escape from apoptosis through FOXO3a-driven glycolytic regulation. Exp Mol Med, 2017. 49: p. e286.

104. Landor, S.K., et al., Hypo- and hyperactivated Notch signaling induce a glycolytic switch through distinct mechanisms. Proc Natl Acad Sci U S A, 2011. 108(46): p. 18814-9.

105. Courtnay, R., et al., Cancer metabolism and the Warburg effect: the role of HIF-1 and PI3K. Mol Biol Rep, 2015. 42(4): p. 841-51.

106. Borggrefe, T., et al., The Notch intracellular domain integrates signals from Wnt, Hedgehog, TGF $/$ BMP and hypoxia pathways. Biochimica et Biophysica Acta (BBA) - Molecular Cell Research, 2016. 1863(2): p. 303313.

107. Gustafsson, M.V., et al., Hypoxia Requires Notch Signaling to Maintain the Undifferentiated Cell State. Developmental Cell, 2005. 9(5): p. 617-628.

108. Carbonaro, M., A. O'Brate, and P. Giannakakou, Microtubule disruption targets HIF-1alpha mRNA to cytoplasmic P-bodies for translational repression. J Cell Biol, 2011. 192(1): p. 83-99.

109. Eliasz, S., et al., Notch-1 stimulates survival of lung adenocarcinoma cells during hypoxia by activating the IGF-1R pathway. Oncogene, 2010. 29(17): p. 2488-98.

110. Kaewpiboon, C., et al., Extract of Bryophyllum laetivirens reverses etoposide resistance in human lung A549 cancer cells by downregulation of NF-kappaB. Oncol Rep, 2014. 31(1): p. 161-8.

111. Meylan, E., et al., Requirement for NF-kappaB signalling in a mouse model of lung adenocarcinoma. Nature, 2009. 462(7269): p. 104-7.

112. Vilimas, T., et al., Targeting the NF-kappaB signaling pathway in Notch1induced T-cell leukemia. Nat Med, 2007. 13(1): p. 70-7. 
113. Espinosa, L., et al., The Notch/Hes1 pathway sustains NF-kappaB activation through CYLD repression in T cell leukemia. Cancer Cell, 2010. 18(3): p. 268-81.

114. Maniati, E., et al., Crosstalk between the canonical NF-kappaB and Notch signaling pathways inhibits Ppargamma expression and promotes pancreatic cancer progression in mice. J Clin Invest, 2011. 121(12): p. 4685-99.

115. Yamamoto, M., et al., NF-kappaB non-cell-autonomously regulates cancer stem cell populations in the basal-like breast cancer subtype. Nat Commun, 2013. 4: p. 2299.

116. Garner, J.M., et al., Constitutive activation of signal transducer and activator of transcription 3 (STAT3) and nuclear factor kappaB signaling in glioblastoma cancer stem cells regulates the Notch pathway. J Biol Chem, 2013. 288(36): p. 26167-76.

117. Ledesma, F.C., et al., A human 5[prime]-tyrosyl DNA phosphodiesterase that repairs topoisomerase-mediated DNA damage. Nature, 2009. 461(7264): p. 674-678.

118. Do, P.M., et al., Mutant p53 cooperates with ETS2 to promote etoposide resistance. Genes Dev, 2012. 26(8): p. 830-45.

119. Esteban, E., M. Casillas, and A. Cassinello, Pemetrexed in first-line treatment of non-small cell lung cancer. Cancer Treat Rev, 2009. 35(4): p. 364-73.

120. Tieche, C.C., et al., Prolonged pemetrexed pretreatment augments persistence of cisplatin-induced DNA damage and eliminates resistant lung cancer stem-like cells associated with EMT. BMC Cancer, 2016. 16: p. 125.

121. Kano, Y., et al., Schedule-dependent interactions between pemetrexed and cisplatin in human carcinoma cell lines in vitro. Oncol Res, 2006. 16(2): p. 85-95.

122. Liang, S.Q., et al., Blocking the epithelial-to-mesenchymal transition pathway abrogates resistance to anti-folate chemotherapy in lung cancer. Cell Death Dis, 2015. 6: p. e1824.

123. Wu, H.M., et al., Gemcitabine-Induced Autophagy Protects Human Lung Cancer Cells from Apoptotic Death. Lung, 2016. 194(6): p. 959-966.

124. Yen, W.C., et al., Targeting Notch signaling with a Notch2/Notch3 antagonist (tarextumab) inhibits tumor growth and decreases tumorinitiating cell frequency. Clin Cancer Res, 2015. 21(9): p. 2084-95.

125. Yabuuchi, S., et al., Notch signaling pathway targeted therapy suppresses tumor progression and metastatic spread in pancreatic cancer. Cancer Lett, 2013. 335(1): p. 41-51.

126. Chong, C.R. and P.A. Janne, The quest to overcome resistance to EGFRtargeted therapies in cancer. Nat Med, 2013. 19(11): p. 1389-400.

127. Sequist, L.V., et al., Genotypic and histological evolution of lung cancers acquiring resistance to EGFR inhibitors. Sci Transl Med, 2011. 3(75): p. $75 \mathrm{ra} 26$. 
128. Saad, N., et al., Epidermal growth factor receptor T790M mutation-positive metastatic non-small-cell lung cancer: focus on osimertinib (AZD9291). OncoTargets and therapy, 2017. 10: p. 1757-1766.

129. Song, H.N., et al., Acquired C797S Mutation upon Treatment with a T790MSpecific Third-Generation EGFR Inhibitor (HM61713) in Non-Small Cell Lung Cancer. J Thorac Oncol, 2016. 11(4): p. e45-7.

130. Arasada, R.R., et al., EGFR blockade enriches for lung cancer stem-like cells through Notch3-dependent signaling. Cancer Res, 2014. 74(19): p. 5572-84.

131. Xie, M., et al., Notch-1 contributes to epidermal growth factor receptor tyrosine kinase inhibitor acquired resistance in non-small cell lung cancer in vitro and in vivo. Eur J Cancer, 2013. 49(16): p. 3559-72.

132. Hu, S., et al., Antagonism of EGFR and Notch limits resistance to EGFR inhibitors and radiation by decreasing tumor-initiating cell frequency. Sci Transl Med, 2017. 9(380).

133. Giannopoulou, E., et al., Epidermal growth factor receptor status and Notch inhibition in non-small cell lung cancer cells. J Biomed Sci, 2015. 22: p. 98.

134. Morgillo, F., et al., Implication of the insulin-like growth factor-IR pathway in the resistance of non-small cell lung cancer cells to treatment with gefitinib. Clin Cancer Res, 2007. 13(9): p. 2795-803.

135. Yeo, C.D., et al., Inhibiting IGF-1R attenuates cell proliferation and VEGF production in IGF-1R over-expressing EGFR mutant non-small cell lung cancer cells. Exp Lung Res, 2017. 43(1): p. 29-37.

136. Reinmuth, N., et al., Correlation of EGFR mutations with chromosomal alterations and expression of EGFR, ErbB3 and VEGF in tumor samples of lung adenocarcinoma patients. Lung Cancer, 2008. 62(2): p. 193-201.

137. Potenta, S., E. Zeisberg, and R. Kalluri, The role of endothelial-tomesenchymal transition in cancer progression. Br J Cancer, 2008. 99(9): $\mathrm{p}$. 1375-9.

138. Noseda, M., et al., Notch Activation Results in Phenotypic and Functional Changes Consistent With Endothelial-to-Mesenchymal Transformation. Circulation Research, 2004. 94(7): p. 910-917.

139. Shiga, K., et al., Cancer-Associated Fibroblasts: Their Characteristics and Their Roles in Tumor Growth. Cancers (Basel), 2015. 7(4): p. 2443-58.

140. Erez, N., et al., Cancer-Associated Fibroblasts Are Activated in Incipient Neoplasia to Orchestrate Tumor-Promoting Inflammation in an $N F-\kappa B$ Dependent Manner. Cancer Cell, 2010. 17(2): p. 135-147.

141. Yoshida, T., et al., Podoplanin-positive cancer-associated fibroblasts in the tumor microenvironment induce primary resistance to EGFR-TKIs in lung adenocarcinoma with EGFR mutation. Clin Cancer Res, 2015. 21(3): p. 64251.

142. Dagogo-Jack, I., A.T. Shaw, and G.J. Riely, Optimizing Treatment for Patients with ALK Positive Lung Cancer. Clin Pharmacol Ther, 2017. 
143. Sakamoto, H., et al., CH5424802, a selective ALK inhibitor capable of blocking the resistant gatekeeper mutant. Cancer Cell, 2011. 19(5): p. 67990.

144. Kogita, A., et al., Hypoxia induces resistance to ALK inhibitors in the H3122 non-small cell lung cancer cell line with an ALK rearrangement via epithelial-mesenchymal transition. Int J Oncol, 2014. 45(4): p. 1430-6.

145. Inai, T., et al., Inhibition of vascular endothelial growth factor (VEGF) signaling in cancer causes loss of endothelial fenestrations, regression of tumor vessels, and appearance of basement membrane ghosts. Am J Pathol, 2004. 165(1): p. 35-52.

146. Tong, R.T., et al., Vascular normalization by vascular endothelial growth factor receptor 2 blockade induces a pressure gradient across the vasculature and improves drug penetration in tumors. Cancer Res, 2004. 64(11): p. 3731-6.

147. Grepin, R. and G. Pages, Molecular mechanisms of resistance to tumour anti-angiogenic strategies. J Oncol, 2010. 2010: p. 835680.

148. Bergers, G. and D. Hanahan, Modes of resistance to anti-angiogenic therapy. Nat Rev Cancer, 2008. 8(8): p. 592-603.

149. Noguera-Troise, I., et al., Blockade of Dll4 inhibits tumour growth by promoting non-productive angiogenesis. Nature, 2006. 444(7122): p. 10321037.

150. Lee, D., et al., Simultaneous blockade of VEGF and Dll4 by HD105, a bispecific antibody, inhibits tumor progression and angiogenesis. MAbs, 2016. 8(5): p. 892-904.

151. Trindade, A., et al., Endothelial Dll4 overexpression reduces vascular response and inhibits tumor growth and metastasization in vivo. BMC Cancer, 2017. 17(1): p. 189.

152. Bhattacharya, S., M.A. Socinski, and T.F. Burns, KRAS mutant lung cancer: progress thus far on an elusive therapeutic target. Clin Transl Med, 2015. 4(1): p. 35.

153. Janne, P.A., et al., Selumetinib Plus Docetaxel Compared With Docetaxel Alone and Progression-Free Survival in Patients With KRAS-Mutant Advanced Non-Small Cell Lung Cancer: The SELECT-1 Randomized Clinical Trial. JAMA, 2017. 317(18): p. 1844-1853.

154. Park, S., et al., KRAS G12C mutation as a poor prognostic marker of pemetrexed treatment in non-small cell lung cancer. Korean J Intern Med, 2017.

155. Maraver, A., et al., Therapeutic effect of gamma-secretase inhibition in KrasG12V-driven non-small cell lung carcinoma by derepression of DUSP1 and inhibition of ERK. Cancer Cell, 2012. 22(2): p. 222-34.

156. Yang, G., et al., Soy food intake and risk of lung cancer: evidence from the Shanghai Women's Health Study and a meta-analysis. Am J Epidemiol, 2012. 176(10): p. 846-55. 
157. Ran, Y., et al., $\gamma$-Secretase inhibitors in cancer clinical trials are pharmacologically and functionally distinct. EMBO Molecular Medicine, 2017.

158. Sullivan, J.P., et al., Aldehyde dehydrogenase activity selects for lung adenocarcinoma stem cells dependent on notch signaling. Cancer Res, 2010. 70(23): p. 9937-48.

159. Liu, J., et al., Blocking the NOTCH pathway can inhibit the growth of CD133-positive A549 cells and sensitize to chemotherapy. Biochem Biophys Res Commun, 2014. 444(4): p. 670-5.

160. Xie, M., et al., Activation of Notch-1 enhances epithelial-mesenchymal transition in gefitinib-acquired resistant lung cancer cells. J Cell Biochem, 2012. 113(5): p. 1501-13.

161. Yang, Y., et al., Pterostilbene exerts antitumor activity via the Notch1 signaling pathway in human lung adenocarcinoma cells. PLoS One, 2013. 8(5): p. e62652.

162. Konishi, J., et al., Notch3 cooperates with the EGFR pathway to modulate apoptosis through the induction of bim. Oncogene, 2010. 29(4): p. 589-96.

163. Zhang, H., et al., Sensitivity of non-small cell lung cancer to erlotinib is regulated by the Notch/miR-223/<em> FBXW7</em> pathway. Bioscience Reports, 2017.

164. Xie, M., et al., gamma Secretase inhibitor BMS-708163 reverses resistance to EGFR inhibitor via the PI3K/Akt pathway in lung cancer. J Cell Biochem, 2015. 116(6): p. 1019-27.

165. Ikezawa, Y., et al., Inhibition of Notch and HIF enhances the antitumor effect of radiation in Notch expressing lung cancer. Int J Clin Oncol, 2017. 22(1): p. 59-69.

166. Sakakibara-Konishi, J., et al., Combined antitumor effect of gammasecretase inhibitor and ABT-737 in Notch-expressing non-small cell lung cancer. Int J Clin Oncol, 2017. 22(2): p. 257-268.

167. Carmeliet, P. and R.K. Jain, Angiogenesis in cancer and other diseases. Nature, 2000. 407(6801): p. 249-57.

168. Meadows, K.L. and H.I. Hurwitz, Anti-VEGF Therapies in the Clinic. Cold Spring Harbor Perspectives in Medicine, 2012. 2(10): p. a006577.

169. Jain, R.K., Normalization of Tumor Vasculature: An Emerging Concept in Antiangiogenic Therapy. Science, 2005. 307(5706): p. 58-62.

170. Gao, X.J., et al., Nobiletin inhibited hypoxia-induced epithelialmesenchymal transition of lung cancer cells by inactivating of Notch-1 signaling and switching on miR-200b. Pharmazie, 2015. 70(4): p. 256-62.

171. Ji, X., et al., Delta-tocotrienol suppresses Notch-1 pathway by upregulating miR-34a in nonsmall cell lung cancer cells. Int J Cancer, 2012. 131(11): p. 2668-77.

172. Wu, G.-Q., et al., Anti-cancer effects of curcumin on lung cancer through the inhibition of EZH2 and NOTCH1. Oncotarget, 2016. 7(18): p. 2653526550. 
173. Mizugaki, H., et al., gamma-Secretase inhibitor enhances antitumour effect of radiation in Notch-expressing lung cancer. Br J Cancer, 2012. 106(12): p. 1953-9.

174. Yahyanejad, S., J. Theys, and M. Vooijs, Targeting Notch to overcome radiation resistance. Oncotarget, 2016. 7(7): p. 7610-28.

175. Yahyanejad, S., et al., NOTCH blockade combined with radiation therapy and temozolomide prolongs survival of orthotopic glioblastoma. Oncotarget, 2016. 7(27): p. 41251-41264.

176. Takebe, N., D. Nguyen, and S.X. Yang, Targeting notch signaling pathway in cancer: clinical development advances and challenges. Pharmacol Ther, 2014. 141(2): p. 140-9.

177. Andersson, E.R. and U. Lendahl, Therapeutic modulation of Notch signalling--are we there yet? Nat Rev Drug Discov, 2014. 13(5): p. 357-78.

178. Pant, S., et al., A first-in-human phase I study of the oral Notch inhibitor, LY900009, in patients with advanced cancer. European Journal of Cancer, 2016. 56: p. 1-9.

179. Diaz-Padilla, I., et al., A phase Ib combination study of RO4929097, a gamma-secretase inhibitor, and temsirolimus in patients with advanced solid tumors. Invest New Drugs, 2013. 31(5): p. 1182-91.

180. Sahebjam, S., et al., A phase I study of the combination of ro4929097 and cediranib in patients with advanced solid tumours (PJC-004/NCI 8503). $\mathrm{Br}$ J Cancer, 2013. 109(4): p. 943-9.

181. Fischer, M., et al., Anti-DLL4 inhibits growth and reduces tumor-initiating cell frequency in colorectal tumors with oncogenic KRAS mutations. Cancer Res, 2011. 71(5): p. 1520-5.

182. Chiorean, E.G., et al., A Phase I First-in-Human Study of Enoticumab (REGN421), a Fully Human Delta-like Ligand 4 (Dll4) Monoclonal Antibody in Patients with Advanced Solid Tumors. Clin Cancer Res, 2015. 21(12): p. 2695-703.

183. Smith, D.C., et al., A Phase I Dose Escalation and Expansion Study of the Anticancer Stem Cell Agent Demcizumab (Anti-DLL4) in Patients with Previously Treated Solid Tumors. Clinical Cancer Research, 2014. 20(24): p. 6295-6303.

184. McKeage, M.J., et al., Phase IB Trial of the Anti-Cancer Stem Cell DLL4Binding Agent Demcizumab with Pemetrexed and Carboplatin as First-Line Treatment of Metastatic Non-Squamous NSCLC. Target Oncol, 2017.

185. Riccio, O., et al., Loss of intestinal crypt progenitor cells owing to inactivation of both Notchl and Notch 2 is accompanied by derepression of CDK inhibitors p27(Kip1) and p57(Kip2). EMBO Reports, 2008. 9(4): p. 377-383.

186. Vooijs, M., et al., Mapping the consequence of Notch1 proteolysis in vivo with NIP-CRE. Development, 2007. 134(3): p. 535-544.

187. Milano, J., et al., Modulation of notch processing by gamma-secretase inhibitors causes intestinal goblet cell metaplasia and induction of genes 
known to specify gut secretory lineage differentiation. Toxicological sciences : an official journal of the Society of Toxicology, 2004. 82(1): p. 341-358.

188. Wong, G., et al., Chronic treatment with the gamma-secretase inhibitor LY411,575 inhibits beta-amyloid peptide production and alters lymphopoiesis and intestinal cell differentiation. J Biol Chem, 2004. 279(13): p. 1287612882.

189. van Es, J.H., et al., Notch/gamma-secretase inhibition turns proliferative cells in intestinal crypts and adenomas into goblet cells. Nature, 2005. 435(7044): p. 959-63.

190. Schott, A.F., et al., Preclinical and clinical studies of gamma secretase inhibitors with docetaxel on human breast tumors. Clin Cancer Res, 2013. 19(6): p. 1512-24.

191. Aguirre, S.A., et al., Intermittent oral coadministration of a gamma secretase inhibitor with dexamethasone mitigates intestinal goblet cell hyperplasia in rats. Toxicol Pathol, 2014. 42(2): p. 422-34.

192. Rangarajan, A., et al., Notch signaling is a direct determinant of keratinocyte growth arrest and entry into differentiation. EMBO J, 2001. 20(13): p. 342736.

193. Proia, T., et al., 23814, an Inhibitory Antibody of Ligand-Mediated Notch1 Activation, Modulates Angiogenesis and Inhibits Tumor Growth without Gastrointestinal Toxicity. Mol Cancer Ther, 2015. 14(8): p. 1858-67.

194. Zheng, H., et al., KLF4 gene expression is inhibited by the notch signaling pathway that controls goblet cell differentiation in mouse gastrointestinal tract. Am J Physiol Gastrointest Liver Physiol, 2009. 296(3): p. G490-8.

195. Real, P.J., et al., Gamma-secretase inhibitors reverse glucocorticoid resistance in T cell acute lymphoblastic leukemia. Nat Med, 2009. 15(1): p. 50-8.

196. Ridgway, J., et al., Inhibition of Dll4 signalling inhibits tumour growth by deregulating angiogenesis. Nature, 2006. 444(7122): p. 1083-7.

197. Pellegrinet, L., et al., Dll1- and Dll4-Mediated Notch Signaling Are Required for Homeostasis of Intestinal Stem Cells. Gastroenterology, 2011.

198. Ozawa, H., et al., Curcumin beta-D-Glucuronide Plays an Important Role to Keep High Levels of Free-Form Curcumin in the Blood. Biol Pharm Bull, 2017. 40(9): p. 1515-1524.

199. Brana, I., et al., A parallel-arm phase I trial of the humanised anti-IGF-1R antibody dalotuzumab in combination with the AKT inhibitor MK-2206, the mTOR inhibitor ridaforolimus, or the NOTCH inhibitor MK-0752, in patients with advanced solid tumours. Br J Cancer, 2014. 111(10): p. 193244.

200. Krop, I., et al., Phase I pharmacologic and pharmacodynamic study of the gamma secretase (Notch) inhibitor MK-0752 in adult patients with advanced solid tumors. J Clin Oncol, 2012. 30(19): p. 2307-13. 
201. Messersmith, W.A., et al., A Phase I, Dose-Finding Study in Patients with Advanced Solid Malignancies of the Oral $\gamma$-Secretase Inhibitor PF03084014. Clinical Cancer Research, 2015. 21(1): p. 60-67.

202. Swift, S.N., et al., Acute toxicity of subcutaneously administered vitamin $E$ isomers delta- and gamma-tocotrienol in mice. Int J Toxicol, 2014. 33(6): $\mathrm{p}$. 450-8.

203. Oh, P., et al., In vivo mapping of notch pathway activity in normal and stress hematopoiesis. Cell Stem Cell, 2013. 13(2): p. 190-204.

204. Yan, M., et al., Chronic DLL4 blockade induces vascular neoplasms. Nature, 2010. 463(7282): p. E6-7.

205. Guseh, J.S., et al., Notch signaling promotes airway mucous metaplasia and inhibits alveolar development. Development, 2009. 136(10): p. 1751-9.

206. Kavian, N., et al., New insights into the mechanism of notch signalling in fibrosis. Open Rheumatol J, 2012. 6: p. 96-102.

207. Okamoto, M., et al., Jaggedl on dendritic cells and Notch on CD4+ T cells initiate lung allergic responsiveness by inducing IL-4 production. J Immunol, 2009. 183(5): p. 2995-3003.

208. Li, X., et al., Notch3 signaling promotes the development of pulmonary arterial hypertension. Nat Med, 2009. 15(11): p. 1289-97.

209. Sannerud, R., et al., Restricted Location of PSEN2/gamma-Secretase Determines Substrate Specificity and Generates an Intracellular Abeta Pool. Cell, 2016. 166(1): p. 193-208.

210. Knoechel, B., et al., An epigenetic mechanism of resistance to targeted therapy in T cell acute lymphoblastic leukemia. Nat Genet, 2014. 46(4): p. 364-70. 


\section{Figure and Tables}

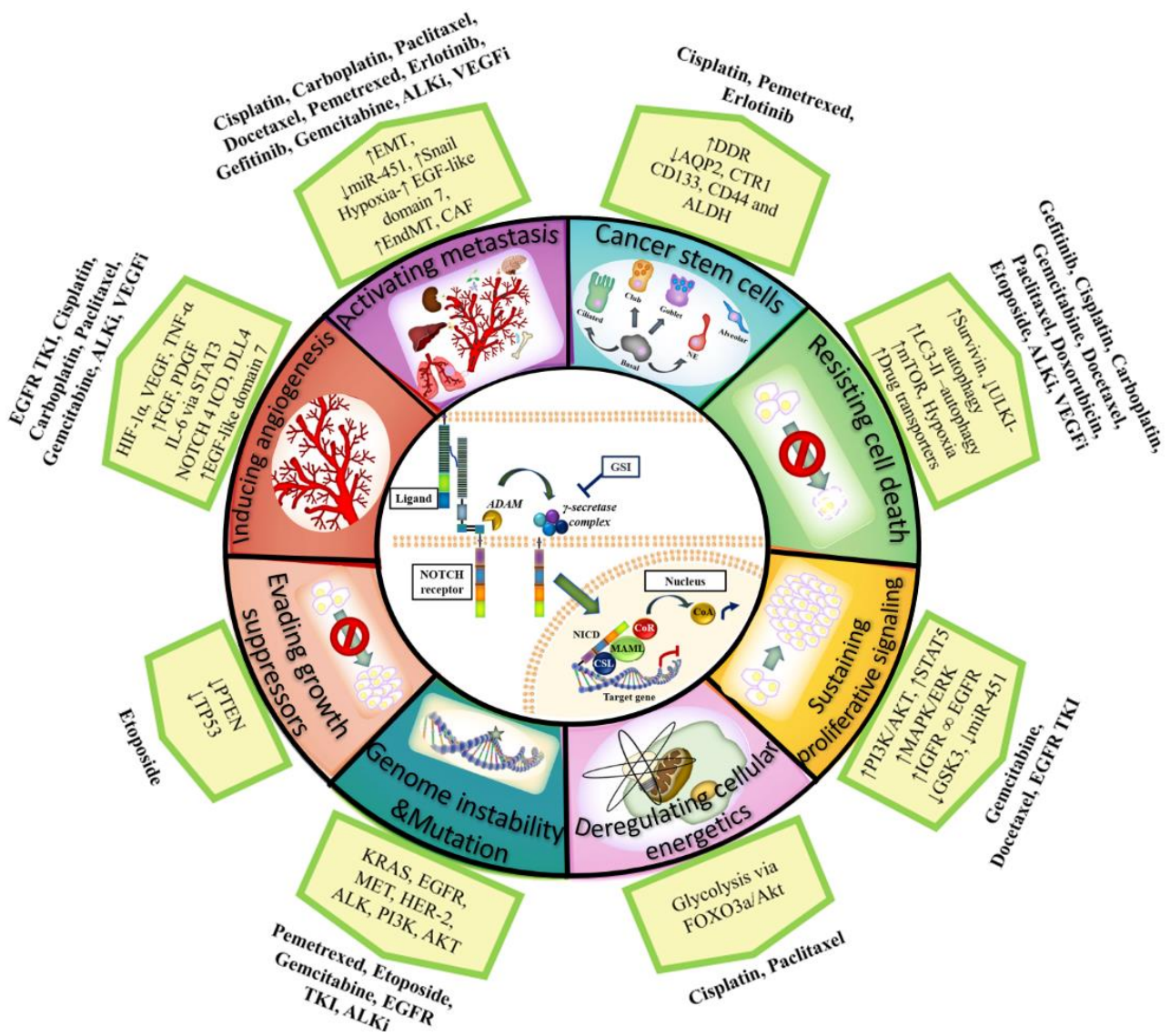

Figure 1: Notch and the Hallmarks of Cancer in tumor resistance to chemotherapy and targeted agents in NSCLC.

Notch1 sustains proliferative signaling by upregulating PI3K/Akt pathway via PTEN repression and induction of IGF-1R under hypoxia. PI3K/Akt and MAPK/ERK/Ras then upregulate HIF- $1 \alpha$ in an oxygen-dependent manner. HIF-1 $\alpha$ binds to N1ICD to regulate each other: HIF-1 $\alpha$ increases gamma-secretase activity to activate Notch signaling whereas factor inhibitor HIF (FIH) hydroxylates and downregulates NICD activity. Notch1 upregulates IGF-1R which forms a heterodimer $(\infty)$ with EGFR and increases survivin (apoptosis inhibitor) to resist cell death. 
Cancer cells also resist cell death by upregulating the autophagosomal marker LC3 nd/or drug transporters (ABCB1 and ABCG2) mediated by Notch-dependent AP1/microRNA-451 or through a glycolysis-associated mechanism via FOXO3a/Akt signaling thus promoting deregulation of cellular energetics. CSL binds to TP53 and they can both repress each other thus evading growth suppressors. Notch signaling also has a role in the maintenance of cancer stem cells. Chemotherapy induces an enrichment of resistant tumor cells expressing cancer stem cell (CSC) markers (CD133, ALDH, CD44). CSC have downregulated the AQP2 and CTR1 drug transporters which prevent drug accumulation and reduce ds DNA damage. Additionally, CSC have increased DNA damage response (DDR) and repair pathways. Notch facilitates metastasis by increasing the epithelial-mesenchymal transition (EMT) via an increase in Twist, Snail, Slug and ZEB. Notch activation can also stimulate endothelial-to-mesenchymal transition (EndMT) which increases the production of cancer-associated fibroblasts (CAF) which are known to be involved in chemotherapy resistance.

DLL4 ligand is positively regulated by proangiogenic factor (VEGF-A, bFGF), IL6 mediated by STAT3 activation, Foxc protein, Notch4 ICD, and HIF- $1 \alpha$ to induce angiogenesis. DLL4 downregulates VEGFR2 to inhibit VEGF-A and endothelial cell proliferation and migration. DLL4 ligand targeting inhibits tumor progression of human lung adenocarcinomas. Upregulated NOTCH signaling activity has been found in cancers with other genetic alterations/mutations (KRAS, EGFR, HER-2, MET, ALK, PI3K) with which it cross-talks. *I: inhibitor, DDR: DNA damage response, miR: micro RNA, GSI: $\gamma$-secretase inhibitor. 


\section{Table 1: Outcome for preclinical trials in non-small cell lung cancer with $\gamma$ - secretase inhibitors (GSI) as monotherapy or in combination with other} chemotherapeutics or targeted agents. SA: Single agent, $\uparrow:$ increase/upregulated, $\downarrow$ : decrease/downregulated, GSI: $\gamma$-secretase inhibitor, GSI?: Unspecified GSI, RT: radiotherapy, neg.: negative, EGFR: Epidermal growth factor receptor, IGF-1R: insulin-like growth factor 1 receptor, DDR1: Discoidin domain receptor 1.

\begin{tabular}{|c|c|c|c|}
\hline \multicolumn{2}{|c|}{ TREATMENT } & \multirow{2}{*}{ OUTCOME } & \multirow{2}{*}{$R E F$} \\
\hline GSI & Combination & & \\
\hline \multirow[b]{2}{*}{ DAPT } & SA & $\begin{array}{ll}\checkmark & \text { Least effective clinical GSI in cleaving NOTCH receptors } \\
\checkmark & \text { NOTCH1 gain-of function /loss of NUMB mutations: sensitive } \\
\checkmark & \uparrow \text { G1/G0 and G2/M arrest } \\
\checkmark & \downarrow \text { ALDH+ cells with } \uparrow \text { NOTCH } 1 / 2 / 3, \text { HEY1/2 and HES } 1 \\
\checkmark & \text { EGFR low/wt cells: } \downarrow \text { proliferation, G0/G1 arrest, } \uparrow \text { Beclin- } 1 \\
\checkmark & \text { Reverts NOTCH-induced EMT phenotype }\end{array}$ & $\begin{array}{l}\text { Ran } Y 2017 \\
\text { Westhoff } \\
\text { 2009; } \\
\text { Giannopoulou } \\
\text { E 2015; } \\
\text { Huang J } \\
\text { 2016; } \\
\text { Sullivan } 2010\end{array}$ \\
\hline & $\begin{array}{l}\text { Cisplatin } \\
\text { Paclitaxel } \\
\text { Doxorubicin } \\
\text { Docetaxel } \\
\text { Gefitinib } \\
\text { Pterostilbene }\end{array}$ & $\begin{array}{ll}\checkmark & \uparrow \text { P-c-Jun, } \uparrow \text { AP-1-regulated miR-451, } \downarrow \text { MDR-1 } \\
\checkmark & \text { Cisplatin-treated cells with } \uparrow \text { drug transporters: sensitive } \\
\checkmark & \downarrow \text { viability of cisplatin-resistant CD133+ cells } \\
\checkmark & \text { Gefitinib-resistant cells: } \uparrow \text { NOTCH1, HES1 and cyclin D1, } \downarrow \text { p21 } \\
& \text { Waf1/Cip1 } \\
\checkmark & \text { GSI }+ \text { cisplatin/docetaxel/gefitinib: in vivo chemosensitization, } \uparrow \\
& \text { G2/M arrest, } \downarrow \text { proliferation, } \uparrow \text { apoptosis } \\
\checkmark & \text { GSI + Pterostilbene: } \downarrow \text { tumor growth } \text { in vivo, } \downarrow \text { pterostilbene-mediated } \\
& \uparrow \text { NICD, HES1 and } \downarrow \text { PI3K/Akt, cyclin D1, surviving, DNA-PK, P- } \\
& \text { mTOR, P-S6 ribosomal protein }\end{array}$ & $\begin{array}{l}\text { Liu YP 2013; } \\
\text { Liu J 2014; } \\
\quad \text { Huang J } \\
2016 ; \text { Xie } M \\
\text { 2013; Xie M } \\
2011 ; \text { Yang Y } \\
\quad 2013\end{array}$ \\
\hline \multirow[t]{2}{*}{ MRK-003 } & SA & $\begin{array}{ll}\checkmark & \text { NOTCH1 gain-of function /loss of NUMB mutations: sensitive } \\
\checkmark & \downarrow \text { tumor formation in H1299 stem-like cells expressing } \uparrow \text { NOTCH } 2 / 4, \\
& \text { HES1, HEY1 resistant to cisplatin/docetaxel, rescued by N1/2 ICD } \\
& \text { (not N3 ICD) in sphere formation } \\
\checkmark & \downarrow \text { NOTCH1-mediated } \uparrow \text { IGFR-1-mediated Akt-1 expression by } \downarrow \\
& \text { PTEN under hypoxia and } \uparrow \text { apoptosis under hypoxia } \\
\checkmark & \downarrow \text { NOTCH3: } \downarrow \text { growth and } \uparrow \text { tumor apoptosis via } \downarrow \text { p-ERK, P-Bcl-2, } \\
\text { Bcl-Xl and } \uparrow \text { Bim, Bax, phospho-BAD proteins }\end{array}$ & $\begin{array}{l}\text { Westhoff B } \\
\text { 2009; Hassan } \\
\text { KA 2013; } \\
\text { Eliasz S 2010; } \\
\text { Konishi J } \\
2010\end{array}$ \\
\hline & $\begin{array}{l}\text { Docetaxel } \\
\text { Dominant neg. } \\
\text { IGFR-1 } \\
\text { Erlotinib }\end{array}$ & $\begin{array}{ll}\checkmark & \text { GSI }+ \text { docetaxel: } \downarrow \downarrow \text { tumor growth } \\
\checkmark & \downarrow \text { IGF-1R sensitizes cells to GSI-induced apoptosis } \\
\checkmark & \text { GSI + erlotinib: } \uparrow \text { ERK-regulated } \uparrow \text { Bim and } \downarrow \text { tumor growth }\end{array}$ & $\begin{array}{l}\text { Hassan KA } \\
\text { 2013; Eliasz S } \\
\text { 2010; Konishi } \\
\text { J } 2010\end{array}$ \\
\hline \multirow{2}{*}{ PF-3084014 } & SA & $\begin{array}{l}\checkmark \text { Preferentially } \downarrow \text { NOTCH2, but also other NOTCH receptors, SPPL } 2 b \text {, } \\
\text { APPC } 100 \text { and APP }\end{array}$ & Ran Y 2017 \\
\hline & Erlotinib & $\begin{array}{ll}\checkmark & \downarrow \text { ALDH+ NOTCH3-dependent cells in EGFR-mutated cell lines } \\
\checkmark & \text { EGFR negatively regulates Notch activity via its TK activity }\end{array}$ & $\begin{array}{l}\text { Arasada RR } \\
\quad 2014\end{array}$ \\
\hline \multirow{2}{*}{ RO4929079 } & SA & $\begin{array}{l}\checkmark \text { Preferentially } \downarrow \text { NOTCH1 followed by NOTCH } 2 / 3, \text { SPPL } 2 b \text { and } \\
\text { APPC } 100\end{array}$ & Ran Y 2017 \\
\hline & Erlotinib & $\begin{array}{ll}\checkmark & \downarrow \text { miR-223, CD44+ erlotinib-resistant cells } \\
\checkmark & \uparrow \text { FBXW7 and reverses erlotinib-resistance }\end{array}$ & Zhang H 2017 \\
\hline \multirow{2}{*}{$\begin{array}{l}\text { BMS- } \\
708163\end{array}$} & SA & $\begin{array}{lll}\checkmark & \downarrow \text { NOTCH1, HES1, PI3K, and AKT (but not mTOR) and Ki67 } \\
\checkmark & \uparrow \text { G1 arrest, active caspase } 3 \text { and PARP }\end{array}$ & \multirow{2}{*}{ Xie M 2014} \\
\hline & Gefitinib & $\begin{array}{ll}\checkmark & \downarrow \text { 3D colony growth, Ki67, gefitinib-resistant tumor xenograft growth } \\
\checkmark & \uparrow \text { cytotoxicity and apoptosis }\end{array}$ & \\
\hline LY-685458 & SA & $\checkmark \quad \downarrow$ NOTCH, DLK1-induced $\uparrow$ MMP9 expression, invasion & LiL 2014 \\
\hline LY-411575 & $\begin{array}{l}\text { DDR1 inhibitor: } \\
\text { 7rh }\end{array}$ & $\begin{array}{l}\checkmark \quad \text { Additive tumor growth delay of KRAS-driven (including TP53-null) } \\
\text { PDX NSCLC and } \uparrow \text { apoptosis } \\
\checkmark \quad \text { Similar therapeutic efficacy to cisplatin/paclitaxel, but displayed } \\
\text { coagulative necrosis, } \downarrow \text { p-Akt and p-p38 }\end{array}$ & $\begin{array}{c}\text { Ambriogio } C \\
2016\end{array}$ \\
\hline GSI XX & $\begin{array}{l}\text { YC-1 HIF inh } \\
\text { RT }\end{array}$ & $\begin{array}{ll}\checkmark & \text { RT-induced HIF-1 } \alpha \uparrow \text { NOTCH3 under hypoxia (reversed by YC-1) } \\
\checkmark & \text { GSI XX 24h-post YC-1 + 8Gy: strongest tumor growth delay in vivo }\end{array}$ & $\begin{array}{l}\text { Ikezawa Y } \\
\quad 2017\end{array}$ \\
\hline GS1 ? & $\begin{array}{l}\text { Cisplatin } \\
\text { ABT-737 (BH3- } \\
\text { only mimetic) }\end{array}$ & $\begin{array}{l}\checkmark \text { NOTCH3: } \downarrow \text { the cisplatin-mediated } \uparrow \text { in spheroid forming } \\
\text { efficiencies, LC3 and } \downarrow \text { ALDHA1, CD44 } \\
\checkmark \quad \text { GSI }+ \text { ABT-737: synergistic } \downarrow \text { proliferation, tumor growth in vivo and } \\
\uparrow \text { Bim and cleaved PARP }\end{array}$ & $\begin{array}{l}\text { Ma Y 2016; } \\
\text { Sakakibara- } \\
\text { Konishi J } \\
\quad 2016\end{array}$ \\
\hline
\end{tabular}


Table 2: Outcome for preclinical trials in non-small cell lung cancer with monoclonal antibodies (mAbs) or natural NOTCH inhibitors (inh) alone or in combination with other chemotherapeutics or targeted agents.

SA: Single agent, $\uparrow:$ increase/upregulated, $\downarrow$ : decrease/downregulated, NSCLC: Nonsmall cell lung cancer, RT: radiotherapy, EGFR: Epidermal growth factor receptor, DDR1: Discoidin domain receptor 1, PDX: Patient-derived xenograft, VEGF: Vascular endothelial growth factor, EMT: Epithelial-Mesenchymal transition, PARP: Poly (ADP-ribose) polymerase, EZH2: Enhancer of zeste homolog 2, APH1: anterior pharynx-defective 1, MMP9: Matrix Metalloprotease 9

\begin{tabular}{|c|c|c|c|c|}
\hline \multicolumn{3}{|c|}{ TREATMENT } & \multirow{2}{*}{ OUTCOME } & \multirow{2}{*}{$\boldsymbol{R E F}$} \\
\hline \multicolumn{2}{|c|}{ NOTCH-based } & Combination & & \\
\hline \multirow{8}{*}{ mAb } & \multirow{2}{*}{$\begin{array}{l}\text { CT16 } \\
\text { (anti-EGFR } \\
\text { and anti- } \\
\text { NOTCH 2/3) }\end{array}$} & SA & $\begin{array}{l}\checkmark \text { tumor initiating capacity upon } \\
\text { reimplantation, tumor growth and reversal of } \\
\text { EMT phenotype } \\
\checkmark \text { Cetuximab and erlotinib-resistant cell lines: } \\
\text { not effective }\end{array}$ & HuS 2017 \\
\hline & & RT & $\begin{array}{l}\checkmark \text { RT-enriched CD133+, EGFR inh.-enriched } \\
\text { ALDH+ } \\
\checkmark \text { the RT-induced EMT (upregulated in } \\
\text { CD133+ but not ALDH+ cells) and DNA } \\
\text { repair genes } \\
\checkmark \text { Prevented tumor regrowth, delayed acquired } \\
\text { resistance to EGFR inhibitors }\end{array}$ & HuS 2017 \\
\hline & \multirow{2}{*}{$\begin{array}{l}\text { Tarextumab } \\
\text { (anti- } \\
\text { NOTCH 2/3) }\end{array}$} & SA & $\checkmark \downarrow$ tumor initiating capacity upon reimplantation & \multirow{2}{*}{ HuS 2017} \\
\hline & & $\begin{array}{l}\text { Erlotinib } \\
\text { Cetuximab }\end{array}$ & $\checkmark$ Additive effect on NOTCH3+ NSCLC PDX & \\
\hline & $\begin{array}{l}\text { HD105 } \\
\text { (anti-DLL4 } \\
\text { and } \\
\text { anti-VEGF) }\end{array}$ & SA & $\begin{array}{l}\checkmark \quad \downarrow \text { cell proliferation, vessel sprouting and } \uparrow \\
\quad \text { apoptosis } \\
\checkmark \quad \downarrow \text { tumor progression in vivo. }\end{array}$ & Lee D 2016 \\
\hline & \multirow{2}{*}{$\begin{array}{l}\text { Murine anti- } \\
\text { DLL4 }\end{array}$} & SA & $\begin{array}{l}\checkmark \uparrow \text { CD31+tumor vessel density } \\
\checkmark \uparrow \text { thin and more branched vessels }\end{array}$ & \multirow{2}{*}{ Lee D 2016} \\
\hline & & $\begin{array}{l}\text { Murine anti- } \\
\text { VEGF antibody }\end{array}$ & $\checkmark \downarrow$ tumor vessel density and functionality & \\
\hline & $\begin{array}{r}\text { Demcizumab } \\
\text { (anti-DLL4) }\end{array}$ & $\begin{array}{l}\text { Abl, Src, c-Kit, } \\
\text { and DDR1 inh. } \\
\text { Dasatinib }\end{array}$ & $\begin{array}{l}\checkmark \text { Durable and better therapeutic efficacy than } \\
\text { cisplatin/paclitaxel in orthotopic PDX KRAS- } \\
\text { driven lung adenocarcinomas }\end{array}$ & $\begin{array}{l}\text { Ambriogio } \\
\text { C } 2016\end{array}$ \\
\hline \multirow{5}{*}{$\begin{array}{l}\text { Natural } \\
\text { inh }\end{array}$} & $\begin{array}{c}\text { Nobiletin } \\
\text { (citrus peels) }\end{array}$ & SA & $\begin{aligned} \checkmark & \uparrow \text { miR-200b under hypoxia } \\
\checkmark & \downarrow \text { NOTCH1, JAGGED1/2, HES } 1 \text { and HEY1 } \\
& \text { (but not NOTCH2/3, nicastrin, presenilin } 1 / 2 \\
& \text { and APH1) independent of gamma-secretase, } \\
& \downarrow \text { invasion in Matrigel } \\
\checkmark & \text { Reversal of hypoxic-induced EMT phenotype }\end{aligned}$ & $\begin{array}{c}\text { Gao XJ } \\
2014\end{array}$ \\
\hline & \multirow{2}{*}{$\begin{array}{c}\text { Delta- } \\
\text { tocotrienol } \\
\text { (blueberries) }\end{array}$} & SA & $\begin{aligned} & \checkmark \downarrow \text { NOTCH1, colony formation and } \\
& \text { invasiveness } \\
& \checkmark \uparrow \text { miR-34a, G0-G1 arrest, apoptosis via p53 } \\
& \text { activation }\end{aligned}$ & Ji X 2012 \\
\hline & & Cisplatin & 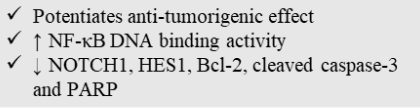 & $\begin{array}{l}\text { Wang J } \\
2012\end{array}$ \\
\hline & $\begin{array}{l}\text { Curcumin } \\
\text { (ginger and } \\
\text { other plants) }\end{array}$ & SA & $\begin{array}{l}\checkmark \text { NOTCH1 by } \downarrow \text { EZH2 via miR-let7c and } \\
\text { miR-101 } \\
\checkmark \text { Delays tumor growth and prevents metastasis }\end{array}$ & $\begin{array}{l}W u G Q \\
2016\end{array}$ \\
\hline & $\begin{array}{l}\text { Delphinidin } \\
\text { (berries) }\end{array}$ & $\begin{array}{l}\text { Bilberry } \\
\text { anthocyanidins }\end{array}$ & $\begin{aligned} & \checkmark \downarrow \text { cell proliferation and migration: } \downarrow \text { NF-kB, } \\
& \text { NOTCH1, } \beta \text {-catenin, c-Myc, MMP9, cyclin } \\
& \text { D1 and B1 } \\
& \checkmark \\
& \checkmark \text { G2/M arrest, apoptosis } \\
& \checkmark \downarrow \text { tumor growth in vivo }\end{aligned}$ & $\begin{array}{c}\text { Kausar } \\
2012\end{array}$ \\
\hline
\end{tabular}


Table 3: Comparison between preclinical in vivo doses of NOTCH-targeted agents used for non-small cell lung cancer treatment.

GSI: $\gamma$-secretase inhibitor, mAb: monoclonal antibody, ip: intraperitoneal, po: per os (oral administration)

\begin{tabular}{|c|c|c|c|}
\hline \multicolumn{2}{|c|}{ TREATMENT } & \multirow{2}{*}{ PRECLINICAL DOSE } & \multirow{2}{*}{$R E F$} \\
\hline Type & Name & & \\
\hline \multirow{6}{*}{ GSI } & DAPT & $\begin{array}{c}8 \mathrm{mg} / \mathrm{kg} \text { ip } 3 \text { days } / \text { week } \\
\text { or } \\
10 \mathrm{mg} / \mathrm{kg} \text { ip once every } 3 \text { days } * 6 \text { injections }\end{array}$ & $\begin{array}{c}\text { Xie M2013; Yang } \\
\text { Y } 2013\end{array}$ \\
\hline & MRK-003 & $150 \mathrm{mg} / \mathrm{kg} 3$ days/week & Konishi J 2010 \\
\hline & BMS-708163 & $10 \mathrm{mg} / \mathrm{kg}$ po 5 days/week & Xie $M 2014$ \\
\hline & LY-411575 & $3 \mathrm{mg} / \mathrm{kg}$ po daily & Ambriogio C 2016 \\
\hline & GSI XX & $200 \mu \mathrm{g} / \mathrm{kg}$ ip 3 days $/$ week $* 2$ cycles & Ikezawa $Y 2017$ \\
\hline & GS1 ? & $200 \mu \mathrm{g} / \mathrm{kg}$ ip 3 days $/$ week $* 2$ cycles & $\begin{array}{l}\text { Sakakibara- } \\
\text { Konishi J } 2016\end{array}$ \\
\hline \multirow{5}{*}{$\mathbf{m A b}$} & $\begin{array}{l}\text { CT16 } \\
\text { (anti-EGFR and anti- } \\
\text { NOTCH 2/3) }\end{array}$ & $40 \mathrm{mg} / \mathrm{kg}$ & Hu S 2017 \\
\hline & $\begin{array}{l}\text { Tarextumab } \\
\quad \text { (anti- } \\
\text { NOTCH 2/3) }\end{array}$ & $40 \mathrm{mg} / \mathrm{kg}$ & HuS 2017 \\
\hline & $\begin{array}{l}\text { HD105 } \\
\text { (anti-DLL4 and } \\
\text { anti-VEGF) }\end{array}$ & $\begin{array}{c}3.25 \mathrm{mg} / \mathrm{kg} \text { ip } 1-2 \text { days } / \text { week } \\
\text { or } \\
6.5 \mathrm{mg} / \mathrm{kg} \text { ip } 1 \text { day } / \text { week }\end{array}$ & Lee D 2016 \\
\hline & Murine anti-DLL4 & $2.5 \mathrm{mg} / \mathrm{kg}$ ip $1-2$ days $/$ week & Lee D 2016 \\
\hline & Demcizumab (anti-DLL4) & $10 \mathrm{mg} / \mathrm{kg}$ ip 1 day/week & Ambriogio C 2016 \\
\hline
\end{tabular}


Table 4: Maximum tolerated dose (MTD) and recommended Phase 2 doses (RP2D) for NOTCH-based therapies in clinical trials for advanced or metastatic solid tumors who no longer respond to or have relapsed from standard therapies. Life-expectancy $\geq 3$ months. ${ }^{*}$ Selected patients with gene/protein alterations in NOTCH pathway. SCC: Squamous cell carcinoma, NSCLC: Nonsmall cell lung cancer, SCLC: Small cell lung cancer, po: per os (oral administration), iv: intravenous, b.i.d.: Bis in die (=2x/day), NR: not reached, MDT: Max dose tested, UD: Undocumented, ONG: Ongoing, Q12H: every $12 \mathrm{~h}, \mathrm{QW}$ : once every week, QD: quaque die (once per day), Q3W: once every 3 weeks, Q2W: once every 2 weeks.

\begin{tabular}{|c|c|c|c|c|c|}
\hline \multicolumn{2}{|c|}{ TREATMENT } & \multirow{2}{*}{ MTD } & \multirow{2}{*}{ RP2D } & \multirow{2}{*}{$\begin{array}{c}\text { TYPE OF CANCER } \\
\text { TESTED }\end{array}$} & \multirow{2}{*}{$\begin{array}{l}\text { Clinical } \\
\text { Trial ID }\end{array}$} \\
\hline Type & Name & & & & \\
\hline \multirow{7}{*}{ GSI } & PF-03084014 & $\begin{array}{l}220 \\
\text { mg po } \\
\text { b.i.d }\end{array}$ & $\begin{array}{c}150 \mathrm{mg} \text { po } \\
\text { b.i.d }\end{array}$ & $\begin{array}{l}\text { lung, colon, desmoid, breast, thyroid, } \\
\text { endometrial, leiomyosarcoma, } \\
\text { pancreas, liver }\end{array}$ & $\begin{array}{c}\text { Messersmith W } \\
2015\end{array}$ \\
\hline & RO4929097 & $\begin{array}{c}\text { NR } \\
\text { (MDT: } \\
20 \mathrm{mg} \\
3 \text { days/ } \\
\text { week) }\end{array}$ & $\begin{array}{c}20 \mathrm{mg} \\
3 \\
\text { days/week }\end{array}$ & $\begin{array}{l}\text { NSCLC, sarcoma, neuroendocrine, } \\
\text { SCC head and neck, pancreas, breast, } \\
\text { colorectal, high-grade glioma, renal, } \\
\text { ovarian, gastrointestinal, stromal, } \\
\text { melanoma, hepatocellular, } \\
\text { endometrial, cholangiocarcinoma }\end{array}$ & $\begin{array}{c}\text { Diaz-PadillaI } \\
\text { 2013; PJC- } \\
004 / N C I 8503 \\
\text { Sahebjam S } \\
2013\end{array}$ \\
\hline & LY900009 & $\begin{array}{c}30 \mathrm{mg} \\
3 \mathrm{x} / \text { week }\end{array}$ & $\begin{array}{l}\text { UD } \\
(<30 \mathrm{mg} \\
3 \mathrm{x} / \text { week })\end{array}$ & $\begin{array}{l}\text { NSCLC, colorectal, endometrial, } \\
\text { ovarian, pancreas, sarcoma, papillary } \\
\text { adenocarcinoma, leiomyosarcoma }\end{array}$ & $\begin{array}{c}\text { NCT01158404 } \\
\text { (Pant S 2016) }\end{array}$ \\
\hline & LY3039478 & \multicolumn{2}{|c|}{$\begin{array}{c}\text { ONG } \\
\text { (MDT: po Q12H) }\end{array}$} & Advanced solid tumors & $\begin{array}{l}\text { NCT02836600; } \\
\text { NCT02784795* }\end{array}$ \\
\hline & BMS-906024 & \multicolumn{2}{|c|}{$\begin{array}{c}\text { ONG } \\
\text { (MDT: } 6 \mathrm{mg} \text { iv QW) }\end{array}$} & $\begin{array}{l}\text { NSCLC, triple-negative breast } \\
\text { cancer, and tumors with proven } \\
\text { active NOTCH }\end{array}$ & $\begin{array}{l}\text { NCT01292655, } \\
\text { NCT01653470 }\end{array}$ \\
\hline & MK-0752 & $\begin{array}{c}\text { UD } \\
\text { (MDT: } \\
4200 \mathrm{mg} \\
\text { po daily) }\end{array}$ & $\begin{array}{c}\text { (UD dose } \\
>1800 \mathrm{mg} \text { ) } \\
\text { po QW } \\
\text { dosing }\end{array}$ & $\begin{array}{l}\text { NSCLC, high grade glioma, } \\
\text { glioblastoma multiforme, anaplastic } \\
\text { astrocytoma, meningioma, } \\
\text { mesothelioma, oligoastrocytoma, } \\
\text { oligodendroglioma, leiomyosarcoma, } \\
\text { bladder, breast, colorectal, kidney, } \\
\text { endometrial, gastrointestinal, head } \\
\text { and neck, melanoma, ovarian, } \\
\text { pancreas, sarcoma, thyroid, } \\
\text { urothelial, stromal }\end{array}$ & $\begin{array}{l}\text { Krop I 2012; } \\
\text { BranaI 2014 }\end{array}$ \\
\hline & BMS-986115 & \multicolumn{2}{|c|}{ UD (MDT: QD) } & Advanced solid tumors & NCT01986218 \\
\hline \multirow{3}{*}{$\begin{array}{c}\text { mAb } \\
\text { anti- } \\
\text { DLL4 }\end{array}$} & Enoticumab & NR & $\begin{array}{c}4 \mathrm{mg} / \mathrm{kg} \\
\mathrm{Q} 3 \mathrm{~W} \text { or } \\
3 \mathrm{mg} / \mathrm{kg} \\
\mathrm{Q} 2 \mathrm{~W}\end{array}$ & $\begin{array}{l}\text { Lung, NSCLC bronchoalveolar-type, } \\
\text { colorectal, ovarian, pancreatic, } \\
\text { sarcoma, breast, salivary gland, head } \\
\text { and neck, thyroid, prostate, } \\
\text { cholangiocarcinoma, hepatocellular }\end{array}$ & $\begin{array}{c}\text { Chiorean EG } \\
2015\end{array}$ \\
\hline & Demcizumab & NR & $\begin{array}{c}5 \mathrm{mg} / \mathrm{kg} \\
\text { Q2W (with } \\
\text { cardio- } \\
\text { protective } \\
\text { agents) }\end{array}$ & $\begin{array}{l}\text { NSCLC, SCLC, colorectal, renal, } \\
\text { pancreatic, salivary gland, breast, } \\
\text { sarcoma, kidney, melanoma, head } \\
\text { and neck, gastric, prostate, bladder, } \\
\text { esophageal, ovarian, testicular/penile, } \\
\text { mesothelioma }\end{array}$ & Smith DC 2014 \\
\hline & MEDI0639 & NR & $\begin{array}{l}\text { Lack of } \\
\text { clinical } \\
\text { activity }\end{array}$ & Advanced solid tumors & NCT01577745 \\
\hline
\end{tabular}


Table 5: Outcome and on-target effects of NOTCH-targeted therapies in clinical trials where lung cancer patients have been included.

GSI: $\boldsymbol{\gamma}$-secretase inhibitor, mAb: monoclonal antibody, inh: inhibitor, SA: Single agent, $\uparrow:$ increase/upregulated, $\downarrow:$ decrease/downregulated, ORR: Objective response rate, OR: Objective response, CR: Complete response, PR: Partial response, DP: Disease progression, adv..: advanced, RP2D: Recommended Phase 2 dose, IC: inconclusive, SD: Stable disease, PFS: Progression free survival, OS: Overall survival, $\infty$ : association, A $\beta$ : amyloid- $\beta$ peptide, NSCLC: Non-small cell lung cancer, po: per os (oral administration), UD: Undocumented, N1/3 ICD: NOTCH 1/3 intracellular domain.

\begin{tabular}{|c|c|c|c|c|c|}
\hline \multicolumn{3}{|c|}{ TREATMENT } & \multirow{2}{*}{ OUTCOME } & \multirow{2}{*}{$\begin{array}{l}\text { ON-TARGET } \\
\text { EFFECTS }\end{array}$} & \multirow{2}{*}{$\begin{array}{l}\text { Clinical } \\
\text { Trial ID }\end{array}$} \\
\hline NOT & H-based & Combination & & & \\
\hline \multirow{6}{*}{ GSI } & PF-03084014 & SA & $\begin{array}{c}\text { ORR } 13 \%: 46 \text { patients } \\
1 \text { CR (adv. thyroid } \\
\text { cancer) } \\
5 / 7 \text { PR (desmoid tumor) } \\
\text { Duration: } 1.74-24 \text { months. }\end{array}$ & $\begin{array}{l}\downarrow H E S 4 \text { in peripheral } \\
\text { blood at RP2D }\end{array}$ & $\begin{array}{l}\text { Messersmith } \\
\quad W 2015\end{array}$ \\
\hline & & SA & $5 / 5 \mathrm{DP}$ & IC & $\begin{array}{c}\text { NCT- } \\
01193868\end{array}$ \\
\hline & RO4929097 & $\begin{array}{l}\text { VEGFRinh } \\
\text { cediranib } \\
\text { or } \\
\text { mTORinh } \\
\text { Temsirolimus }\end{array}$ & $\begin{array}{c}1 \mathrm{PR} \text { at best } \\
11 / 17-19 \mathrm{SD} \\
\text { 7/19DP } \\
\text { PFS: } 4.2 \text { months }\end{array}$ & $\begin{array}{c}\text { No } \infty \text { NOTCH } \\
\text { biomarkers and time to } \\
\text { progression at RP2D }\end{array}$ & $\begin{array}{l}\text { Diaz-PadillaI } \\
\text { 2013; PJC- } \\
\text { 004/NCI } 8503 \\
\text { Sahebjam S } \\
2013\end{array}$ \\
\hline & LY900009 & SA & $\begin{array}{c}\text { No OR } \\
\text { 5/35 SD (NSCLC, } \\
\text { papillary adenocarcinoma, } \\
\text { leiomyosarcoma, ureter } \\
\text { and rectal carcinoma) }\end{array}$ & $\begin{array}{c}\downarrow A \beta \text { in plasma } \\
1 / 35 \uparrow \text { glandular mucin }\end{array}$ & $\begin{array}{c}\text { NCT- } \\
\text { O1158404 } \\
(\text { Pant S 2016) }\end{array}$ \\
\hline & MK-0752 & SA & $\begin{array}{c}1 \mathrm{CR} \geq 1 \text { year (anaplastic } \\
\text { astrocytoma) } \\
12 \mathrm{SD} \geq 4 \text { months (high- } \\
\text { grade glioma) } \\
\text { No clinical activity for } \\
\text { extracranial tumors }\end{array}$ & $\begin{array}{l}\downarrow \text { NOTCH in hair } \\
\text { follicles } \\
(1800-4200 \mathrm{mg} \text { po } \\
\text { lx/week) }\end{array}$ & Krop I 2012 \\
\hline & & $\begin{array}{l}\text { Dalotuzumab } \\
\text { anti-IGFR1 }\end{array}$ & $12 / 12 \mathrm{DP}$ & UD & Brana 12014 \\
\hline \multirow{3}{*}{$\begin{array}{c}\text { mAb } \\
\text { anti- } \\
\text { DLL4 }\end{array}$} & Enoticumab & SA & $\begin{array}{c}\text { 2/44 PR (NSCLC } \\
\text { bronchoalveolar-type and } \\
\text { ovarian cancer) } \\
16 / 44 \text { SD ( } 3 \text { patients } \geq 6 \\
\text { months) } \\
1 \text { DP (low titer anti- } \\
\text { enoticumab antibodies) }\end{array}$ & $\begin{array}{l}\text { In 39/40 tumors } \\
\text { - DLL4+ and CD31+ } \\
\text { - } 30 \% \text { VEGFR-2+ } \\
\text { vessels } \infty \text { N1 and N3 } \\
\text { ICD in tumor, } \\
\text { NOTCH3 and DLL4 in } \\
\text { vessels } \\
\text { - No } \infty \text { NOTCH } \\
\text { biomarkers and time to } \\
\text { progression }\end{array}$ & $\begin{array}{c}\text { Chiorean EG } \\
2015\end{array}$ \\
\hline & Demcizumab & SA & $\begin{array}{c}21 / 48 \mathrm{SD} \text { (NSCLC, renal } \\
\text { and colorectal } \\
\text { carcinomas) } \\
16 / 25(10 \mathrm{mg} / \mathrm{kg}): \mathrm{SD} \text { or } \\
\mathrm{PR} \\
6 / 55 \text { anti-demcizumab } \\
\text { antibodies. No impact on } \\
\text { biological activity. }\end{array}$ & $\begin{array}{l}\text { In blood: } \\
\text { • } \downarrow \text { NOTCH and WNT } \\
\text { pathway genes } \\
\text { (HEY1/2, SPON2, } \\
\text { CCNA2) } \\
\text { - } \uparrow \text { Negative regulators } \\
\text { (AP2A1, AP2B1, } \\
\text { SFRP2, FOXO3, } \\
\text { CDH1, ANK1, } \\
\text { BCL2L1) angiogenesis } \\
\text { genes } \\
\text { In hair follicles: } \\
\text { - } \downarrow \text { Stem cell genes } \\
\text { - } \uparrow \text { vascular genes }\end{array}$ & $\begin{array}{l}\text { Smith DC } \\
2014\end{array}$ \\
\hline & MEDI0639 & SA & $\begin{array}{c}1 \mathrm{PR} \text { for } 1.3 \text { months } \\
9 / 25 \mathrm{SD} \text { for } 5.9 \text { months } \\
12 / 25 \mathrm{DP} \\
\text { PFS } \leq 7.1 \text { months } \\
\text { OS } \leq 28.9 \text { months }\end{array}$ & IC & $\begin{array}{c}\text { NCT- } \\
01577745\end{array}$ \\
\hline
\end{tabular}


Table 6: Associated toxicities of NOTCH-based therapies used preclinically for non-small cell lung cancer.

GSI: $\gamma$-secretase inhibitor, mAb: monoclonal antibody, ip: intraperitoneal, po: per os (oral administration), sc: subcutaneous, iv: intravenous, QW: once every week, $\uparrow$ : increase/upregulated, $\downarrow$ : decrease/downregulated, DLT: Dose-limiting toxicity

\begin{tabular}{|c|c|c|c|c|}
\hline \multicolumn{2}{|c|}{ TREATMENT } & \multirow{2}{*}{ DOSE } & \multirow{2}{*}{ TOXICITY } & \multirow{2}{*}{$\boldsymbol{R E F}$} \\
\hline Type & Name & & & \\
\hline \multirow{8}{*}{ GSI } & MRK-003 & $\begin{array}{l}100 \mathrm{mg} / \mathrm{kg} \text { po } 3 \\
\text { days }\end{array}$ & $\checkmark$ Diarrhea, dehydration, severe weight loss & $\begin{array}{l}\text { Schott } \\
2013\end{array}$ \\
\hline & \multirow[b]{2}{*}{ PF-3084014 } & $\begin{array}{l}150 \mathrm{mg} / \mathrm{kg} \text { po } 7 \\
\text { days }\end{array}$ & $\begin{array}{ll}\checkmark & \text { Goblet cell hyperplasia } \\
\checkmark & \downarrow \text { total whole blood count, lymphocyte, basophils, } \\
& \text { eosinophils, leukocytes } \\
\checkmark & \text { Mild jejunal eosinophilic inflammation }\end{array}$ & \multirow[b]{2}{*}{$\begin{array}{l}\text { Aguirre } \\
2014\end{array}$} \\
\hline & & $\begin{array}{l}100 \mathrm{mg} / \mathrm{kg} \mathrm{GSI}, 3- \\
\text { weeks*2 cycles } \\
1.0 \mathrm{mg} / \mathrm{kg} \\
\text { dexamethasone } \\
\text { weeks } 1 \text { and } 3\end{array}$ & $\begin{array}{l}\checkmark \text { Dexamethasone ameliorates gastrointestinal effects } \\
\text { (DLT) }\end{array}$ & \\
\hline & BMS-708163 & $\begin{array}{l}6 \mathrm{ml} / \mathrm{kg} \text { in } \\
\text { polyethylene } \\
\text { glycol po } 14 \text { days }\end{array}$ & $\begin{array}{ll}\checkmark & \uparrow \text { liver weights } \\
\checkmark & \text { No gastrointestinal lesions nor differentiation } \\
& \text { abnormalities } \\
\checkmark & \text { No significant immune effects }\end{array}$ & $\begin{array}{c}\text { Barten } \\
2005\end{array}$ \\
\hline & LY-411575 & $\begin{array}{l}1-10 \mathrm{mg} / \mathrm{kg} \text { po } \\
5 \text { or } 15 \text { days }\end{array}$ & $\begin{array}{l}\checkmark \quad \text { Altered lymphocyte development, thymus atrophy, } \\
\text { body weight } \\
\checkmark \quad \uparrow \text { goblet cell number, secretion of mucin into the } \\
\text { intestinal lumen, epithelial erosion, infiltration of } \\
\text { inflammatory cells in the lamina propria and necrosis } \\
\checkmark \quad \text { skin: epidermal/epithelial hyperplasia, } \\
\text { follicular/epidermal inclusion cysts }\end{array}$ & $\begin{array}{l}\text { Wong } \\
\text { 2004, } \\
\text { Rangaraj } \\
\text { an } 2001\end{array}$ \\
\hline & GSI & $10 \mu \mathrm{mol} / \mathrm{kg} 1$ day & $\begin{array}{l}\checkmark \text { Gastrointestinal mucous metaplasia, conversion of } \\
\text { cryptal cells into goblet cells (goblet cell hyperplasia) }\end{array}$ & $\begin{array}{l}\text { Zheng } \\
2009\end{array}$ \\
\hline & \multirow[t]{2}{*}{ DBZ } & $\begin{array}{l}\quad 2.5 \mathrm{ml} / \mathrm{kg} \text { ip } 5 \\
\quad \text { days } \\
\checkmark 3-30 \mu \mathrm{mol} / \mathrm{kg} \text { ip } \\
5 \text { days } \\
\checkmark \quad 10 \mu \mathrm{mol} / \mathrm{kg} \\
\quad \text { daily } 4 \text { weeks }\end{array}$ & $\begin{array}{ll}\checkmark & \text { Distension of the stomach and intestine } \\
\checkmark & \uparrow \text { mucous, goblet cell metaplasia (duodenum, jejunum) } \\
\text { and hyperplasia, apoptosis (small intestinal crypt } \\
\text { epithelial cells, large intestinal glands), villus atrophy } \\
\text { and severe diarrhea } \\
\checkmark \quad \text { splenic marginal zone lymphoid tissue atrophy }\end{array}$ & $\begin{array}{l}\text { Milano } \\
2004, \\
\text { Johan H. } \\
\text { van Es } \\
2005 \\
\text { Proia } \\
2015\end{array}$ \\
\hline & & $\begin{array}{l}\text { DBZ } 10 \mu \mathrm{mol} / \mathrm{kg} \\
\text { ip } \\
\text { Dexamethasone } \\
15 \mathrm{mg} / \mathrm{kg} \text { ip } \\
\text { Daily } 5 \text { or } 10 \text { days }\end{array}$ & $\begin{array}{l}\checkmark \quad \text { DBZ: } \uparrow \text { intestinal secretory metaplasia, goblet cell } \\
\text { hyperplasia and } \downarrow \text { proliferation small intestine crypt } \\
\text { cells } \\
\checkmark \quad \text { Combination: normal goblet cell numbers and tissue } \\
\text { architecture of intestinal epithelium }\end{array}$ & Real 2009 \\
\hline \multirow{3}{*}{ mAb } & $\begin{array}{l}\text { OMP59R5 } \\
\quad(\text { anti- } \\
\text { NOTCH 2/3) }\end{array}$ & $\begin{array}{l}40 \mathrm{mg} / \mathrm{kg} \text { every } \\
\text { other day }\end{array}$ & $\begin{array}{ll}\checkmark & \text { Minimal intestinal toxicity } \\
\checkmark & \text { Rodent teeth affectance (long-term repeated high } \\
& \text { doses) }\end{array}$ & Yen 2015 \\
\hline & $\begin{array}{l}\text { HD105 } \\
\text { (anti-DLL4 and } \\
\text { anti-VEGF) }\end{array}$ & $\begin{array}{l}1-3 \mathrm{mg} / \mathrm{kg} \mathrm{QW} \text { for } \\
\quad 8 \text { weeks } \\
10 \mathrm{mg} \text { every } 3 \text { days } \\
(5 \text { doses, } 30 \mathrm{mg} / \mathrm{kg})\end{array}$ & $\begin{array}{ll}\checkmark & \uparrow \text { activation of endothelial cells } \\
\checkmark & \text { Sinusoidal dilation } \\
\checkmark & \text { Centrilobular hepatocyte atrophy }\end{array}$ & $\begin{array}{l}\text { Yan } M \\
2009\end{array}$ \\
\hline & anti-DLL4 & $10 \mathrm{mg} / \mathrm{kg} 2$ weeks & $\checkmark \quad$ No impact on intestinal goblet cell differentiation & $\begin{array}{l}\text { Ridgway } \\
2006\end{array}$ \\
\hline \multirow{2}{*}{$\begin{array}{l}\text { Natural } \\
\text { agents }\end{array}$} & $\begin{array}{l}\text { Delta- } \\
\text { tocotrienol } \\
\text { (blueberries) }\end{array}$ & $\begin{array}{l}200-800 \mathrm{mg} / \mathrm{kg} \mathrm{sc} \\
14-30 \text { days }\end{array}$ & $\begin{array}{l}\checkmark \quad \begin{array}{l}\text { Dose-dependent severity (up to moderately severe) of } \\
\text { dermatitis and inflammation }\end{array} \\
\checkmark \quad \begin{array}{l}\text { No adverse effects were observed in any tissues or } \\
\text { organs }\end{array}\end{array}$ & Swift 2014 \\
\hline & $\begin{array}{l}\text { Curcumin } \\
\text { (ginger and } \\
\text { other plants) }\end{array}$ & $\begin{array}{l}\text { iv } 14 \text { days: } \\
\checkmark \quad 250 \mathrm{mg} / \mathrm{kg} \\
\checkmark \quad 500-1000 \\
\quad \mathrm{mg} / \mathrm{kg}\end{array}$ & 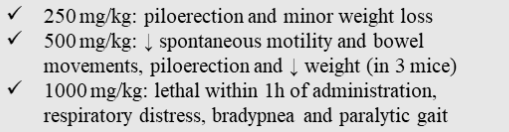 & $\begin{array}{l}\text { Ozawa } \\
2017\end{array}$ \\
\hline
\end{tabular}


Table 7: Toxicity and Pharmacokinetics (PK) of NOTCH-targeted therapies in clinical trials where lung cancer patients have been included.

** Better symptoms than those for RO4929097 and MK0752; ***Better GI toxicity than GSI. GSI: $\gamma$-secretase inhibitor, mAb: monoclonal antibody, inh: inhibitor, SA: Single agent, ID: identifier, $T_{1 / 2}$ : Half-life, UD: Undocumented, CL: Clearance, $\infty$ : association, Q3W: once every 3 weeks, Q2W: once every 2 weeks, DLT: Doselimiting toxicity, [Conc $]_{\text {Max }}$ : Maximum observed concentration, AUC: Area Under the Concentration-Time Curve

\begin{tabular}{|c|c|c|c|c|c|}
\hline \multicolumn{3}{|c|}{ TREATMENT } & \multirow{2}{*}{ TOXICITY } & \multirow{2}{*}{ PK } & \multirow{2}{*}{$\begin{array}{l}\text { Clinical } \\
\text { Trial ID }\end{array}$} \\
\hline \multicolumn{2}{|c|}{ NOTCH-based } & Combination & & & \\
\hline \multirow{7}{*}{ GSI } & PF-03084014 & SA & $\begin{array}{c}\text { Manageable } \\
\text { gastrointestinal adverse } \\
\text { events** }\end{array}$ & $\begin{array}{l}\mathrm{T}_{1 / 2}: 22-40 \mathrm{~h} \text { after } \\
\text { multiple dosing }\end{array}$ & $\begin{array}{l}\text { Messersmith } \\
\text { W } 2015\end{array}$ \\
\hline & \multirow[t]{3}{*}{ R04929097 } & SA & $\begin{array}{c}\text { Serious adverse events in } \\
\geq 1 / 5 \text { patients: } \\
\cdot \text { small intestine } \\
\text { obstruction, constipation, } \\
\text { nausea } \\
\text { - lung infection/sepsis, } \\
\text { dyspnea } \\
\text { Cardiac arrest, } \\
\text { tachycardia }\end{array}$ & UD & $\begin{array}{c}\text { NCT-0119386; } \\
\text { NCT- } \\
01217411\end{array}$ \\
\hline & & $\begin{array}{l}\text { VEGFRinh } \\
\text { cediranib }\end{array}$ & $\begin{array}{l}\text { Grade III-IV: diarrhea, } \\
\text { headache, hypertension, } \\
\text { nausea, hypothyroidism, } \\
\text { hypophosphatemia }\end{array}$ & $\begin{array}{l}\text { Combination didn’t } \\
\text { affect PK profile }\end{array}$ & \multirow{2}{*}{$\begin{array}{c}\text { Diaz-PadillaI } \\
2013 ; \text { PJC- } \\
004 / \text { NCI } 8503 \\
\text { Sahebjam S } \\
2013\end{array}$} \\
\hline & & $\begin{array}{l}\text { mTORinh } \\
\text { Temsirolimus }\end{array}$ & Grade III: rash, mucositis & $\begin{array}{l}\text { RO4929097 induces } \\
\text { CYP3A4: } \\
\uparrow \text { Temsirolimus CL }\end{array}$ & \\
\hline & LY900009 & SA & $\frac{\text { Grade III mucosal }}{\text { inflammation }}$ & $\begin{array}{c}\text { Absorption: } 1-4 \mathrm{~h} \\
\text { Elimination } \mathrm{T}_{1 / 2}: 2-4 \mathrm{~h} \\
\text { LSN2831047(GSI } \\
\text { metabolite): } \\
\text { Appearance: } 2-6 \mathrm{~h} \\
\mathrm{~T}_{1 / 2}: 5-14 \mathrm{~h}\end{array}$ & $\begin{array}{c}N C T- \\
01158404 \\
(\text { Pant } S 2016)\end{array}$ \\
\hline & & SA & $\begin{array}{l}\text { Weekly dosing was } \\
\text { generally well tolerated }\end{array}$ & $\begin{array}{l}\text { Slow absorption } \\
\text { half-life: } 15 \mathrm{~h}\end{array}$ & Krop I 2012 \\
\hline & MK-0752 & $\begin{array}{l}\text { Dalotuzumab } \\
\text { anti-IGFR1 }\end{array}$ & $\begin{array}{l}\text { Grade III dehydration, } \\
\text { rash and diarrhea }\end{array}$ & $\begin{array}{c}\cdot \mathrm{MK}-07521.63-8 \\
\mu \mathrm{mol} / \mathrm{l} \text { in plasma } \\
\cdot \text { Dalotuzumab 34-64 } \\
\mu \mathrm{g} / \mathrm{ml} \text { in serum (at day } \\
\text { 8), accumulated in } \\
\text { time. }\end{array}$ & BranaI 2014 \\
\hline \multirow{3}{*}{$\begin{array}{c}\text { mAb } \\
\text { anti- } \\
\text { DLL4 }\end{array}$} & $\begin{array}{c}\text { Enoticumab } \\
(* * *)\end{array}$ & SA & $\begin{array}{c}\text { Grade III }(0.5 \mathrm{mg} / \mathrm{kg} \\
\text { Q3W): nausea } \\
\text { Grade III }(1 \mathrm{mg} / \mathrm{kg} \\
\text { Q2W): } \\
\text { abdominal pain } \\
\text { Severe effects in } 4 \\
\text { patients: ventricular } \\
\text { dysfunction and } \\
\text { pulmonary hypertension }\end{array}$ & $\begin{array}{c}\text { Nonlinear PK. } \\
\mathrm{T}_{1 / 2}: 8-9 \text { days } \\
\text { Dose-independent CL } \\
(\text { dose }>1.5 \mathrm{mg} / \mathrm{kg}) \\
>2 \mathrm{mg} / \mathrm{L} \text { in plasma } \infty \\
\text { max. tumor activity }\end{array}$ & $\begin{array}{c}\text { Chiorean EG } \\
2015\end{array}$ \\
\hline & Demcizumab & SA & $\begin{array}{c}\text { Generally well tolerated } \\
\text { at doses } \leq 5 \mathrm{mg} \text { weekly. } \\
4 \text { patients }(10 \mathrm{mg} / \mathrm{kg} \\
\text { Q2W): congestive heart } \\
\text { failure } \\
\text { Not more than one DLT } \\
\text { per dose level. }\end{array}$ & $\begin{array}{l}\text { PK within linear range } \\
\text { CL: } 4.17 \mathrm{ml} / \text { day } / \mathrm{kg} \\
\mathrm{T}_{1 / 2}: 15.9 \text { days } \\
(>10 \mu \mathrm{g} / \mathrm{ml})\end{array}$ & Smith DC 2014 \\
\hline & MEDI0639 & SA & No participants with DLT & $\begin{array}{c}\text { AUC: } 7.4- \\
512 \mu \mathrm{g} / \mathrm{day} / \mathrm{ml} \\
{[\mathrm{Conc}]_{\mathrm{Max}} \text { in blood: }} \\
3.2-81.6 \mu \mathrm{g} / \mathrm{ml} \\
\mathrm{CL}: 1.4-0.5 \mathrm{~L} / \text { day } \\
\mathrm{T}_{1 / 2}: 1.5-8.25 \text { days }\end{array}$ & $\begin{array}{c}N C T- \\
01577745\end{array}$ \\
\hline
\end{tabular}




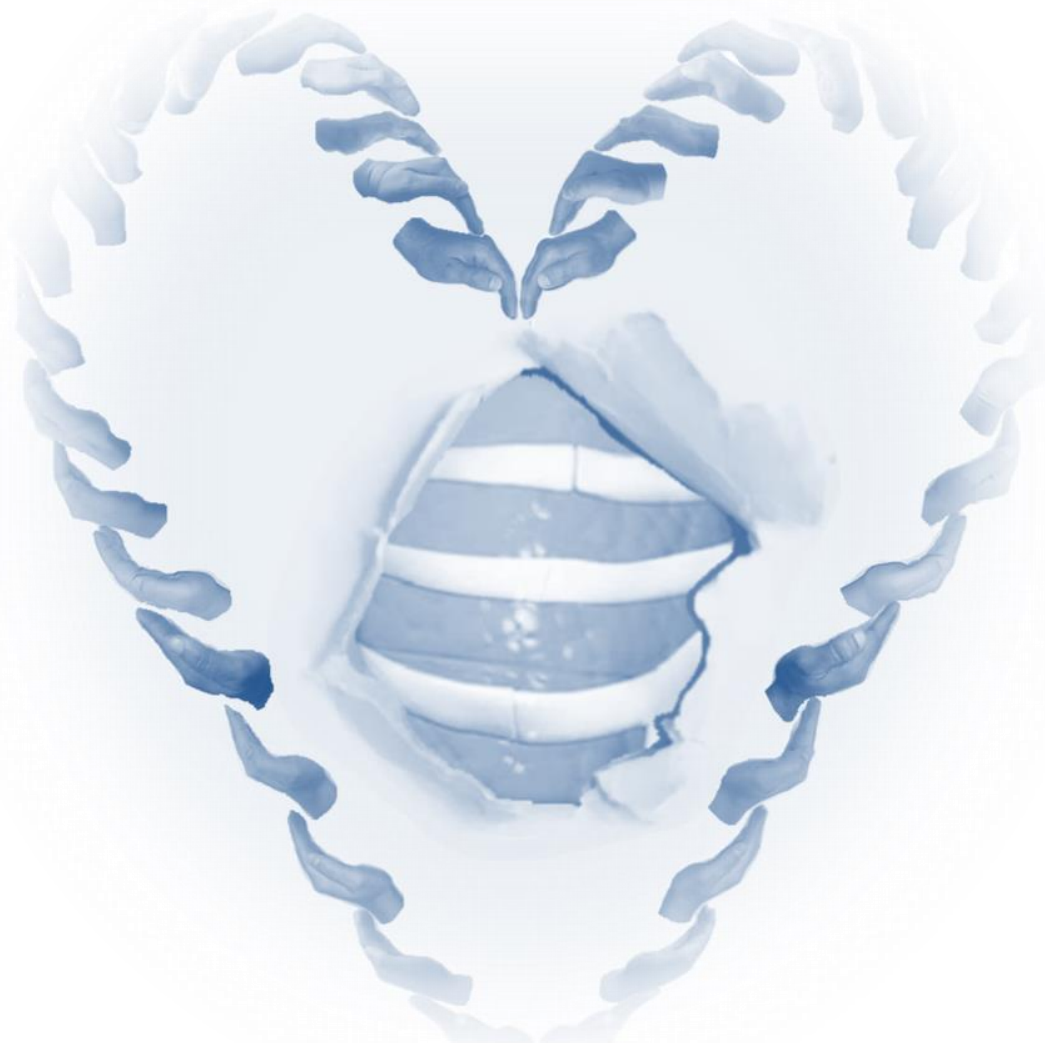




\section{CHAPTER}

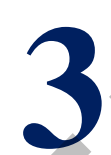

NOTCH inhibition enhances synergistically the efficacy of conventional chemotherapeutics and chemoradiation in non-squamous non-small cell lung cancer cell lines

Venus Sosa Iglesias ${ }^{1}$, Jan Theys ${ }^{1}$, Lydie Barbeau ${ }^{1}$, Alyssa Lemmens $^{1}$, Ala Yaromina ${ }^{1}$, Mario Losen ${ }^{2}$, Ruud Houben ${ }^{1,3}$, Arjan Groot ${ }^{1}$, Ludwig Dubois ${ }^{1}$, Marc Vooijs ${ }^{1}$.

1. Department of Radiation Oncology (MaastRO),

GROW - School for Oncology \& Developmental Biology

Maastricht University Medical Hospital (MUMC)

2. Department of Psychology and Neuropsychology, MHeNS, MUMC

3. MaastRO Clinic

Maastricht, The Netherlands

Submitted to BMC Cancer 


\section{CHAPTER

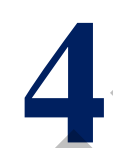

NOTCH inhibition to enhance cisplatin and

radiation treatments in human NSCLC xenografts

Venus Sosa Iglesias ${ }^{1}$, Lydie Barbeau ${ }^{1}$, Natasja Lieuwes ${ }^{1}$, Sanaz

Yahyanejad $^{1}$, Arjan Groot ${ }^{1}$, Marion Gijbels ${ }^{2}$, Jan Bussink ${ }^{3}$, Ludwig J. Dubois ${ }^{1 x}$, Jan Theys ${ }^{1 X}$, Marc Vooijs ${ }^{1}$.

\section{X: Equal contributors}

1. Department of Radiation Oncology (MaastRO), GROW - School for Oncology \& Developmental Biology Maastricht University Medical Hospital (MUMC).

Maastricht, The Netherlands.

2. Departments of Pathology (CARIM, MUMC), and of Molecular Genetics, Maastricht University.

Maastricht, The Netherlands.

3. Department of Radiation Oncology, Radboud University.

Nijmegen, the Netherlands 


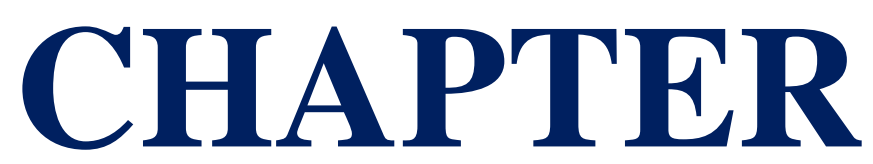

\section{A novel clinically-relevant orthotopic non-small cell lung cancer model for image-guided small animal radiotherapy platforms}

Venus Sosa Iglesias ${ }^{1}$, Stefan J van Hoof ${ }^{2}$, Lotte E J R Schyns ${ }^{2}$, Ana Vaniqui ${ }^{2}$, Relinde Lieverse ${ }^{2}$, Natasja Lieuwes ${ }^{1}$, Ala Yaromina ${ }^{1}$, Linda Spiegelberg ${ }^{1}$, Arjan Groot ${ }^{1}$, Frank Verhaegen $^{2}$, Jan Theys ${ }^{1}$, Ludwig J Dubois ${ }^{1}$, Marc Vooijs ${ }^{1}$.

1. Department of Radiation Oncology (MaastRO), GROW - School for Oncology \& Developmental Biology Maastricht University Medical Hospital (MUMC)

2. MaastRO Clinic

Maastricht, The Netherlands

Extended version of the

Submitted manuscript to British Journal of Radiology 


\section{CHAPTER 6}

\section{NOTCH blockade combined with radiation therapy and temozolomide prolongs survival of orthotopic glioblastoma xenografts}

Sanaz Yahyanejad ${ }^{1}$, Henry King ${ }^{2}$, Venus Sosa Iglesias ${ }^{1}$, Patrick V. Granton ${ }^{3,4}$, Lydie M.O. Barbeau ${ }^{1}$, Stefan J. van Hoof ${ }^{4}$, Arjan J. Groot ${ }^{1}$, Roger Habets ${ }^{1}$, Jos Prickaerts ${ }^{5}$, Anthony J. Chalmers ${ }^{6}$, Jan Theys ${ }^{1}$, Susan C. Short ${ }^{2}$, Frank Verhaegen ${ }^{4}$, Marc Vooijs ${ }^{1}$

1. Department of Radiation Oncology (MaastRO), School for Oncology \& Developmental Biology (GROW)

Maastricht University Medical Hospital (MUMC). Maastricht, The Netherlands.

2. Radiation Biology and Therapy Group, Leeds Institute of Cancer and Pathology. St James's University Hospital. Leeds, England.

3. Department of Oncology, London Health Sciences Center.

London, Ontario, Canada.

4. MaastRO Clinic, Maastricht, The Netherlands.

5. Department of Psychiatry and Neuropsychology. Maastricht University. Maastricht, The Netherlands.

6. Translational Radiation Biology, Institute of Cancer Sciences. Wolfson Wohl Cancer Research Centre, University of Glasgow. Glasgow, Scotland.

Published in Oncotarget 2016; 7(27):41251-41264. 


\section{TABLE OF CONTENTS}

$\begin{array}{ll}\text { AbSTRACT } & 207\end{array}$

$\begin{array}{ll}\text { INTRODUCTION } & 207\end{array}$

MATERIALS AND METHODS $\quad 208$

- Cell line Culture 208

- ORTHOTOPIC BRAIN TUMOR IMPLANTATION AND DRUG TREATMENT 209

- IN VIVO BIOLUMINESCENCE IMAGING (BLI) 209

- IN VIVO IRRADIATION: SMART-PLAN.

- 3D SPHEROID ASSAY 210

- Proliferation assay 211

- Quantitative Real Time PCR (QPCR) 211

- Clonogenic SURVIVAl ASSAY 211

- Flow CYTOMETRY 212

- WESTERN BLOTTING 212

- Statistical ANAlysis 212

RESULTS

- NOTCH PATHWAY IS ACTIVE IN GBM CELLS 213

- NOTCH INHIBITION IN COMBINATION WITH RT AND TMZ ATTENUATES PROLIFERATION AND CLONOGENIC SURVIVAL IN VITRO 213

- NOTCH INHIBITION COMBINED WITH STANDARD OF CARE TREATMENT REDUCES TUMOR GROWTH AND PROLONGS SURVIVAL OF INTRACRANIAL GBM IN VIVO 214

- COMBINATION TREATMENT (GSI + TMZ + RT) REDUCES THE STEM CELL POPULATION 216

DISCUSSION 216

ACKNOWLEDGEMENTS $\quad 220$

CONFLICT OF INTEREST $\quad 220$

REFERENCES $\quad 221$

FIGURES AND TABLES

$\begin{array}{ll}\text { SUPPLEMENTARY INFORMATION } & 231\end{array}$ 


\section{Abstract}

Glioblastoma multiforme (GBM) is the most common malignant brain tumor in adults. The current standard of care includes surgery followed by radiotherapy (RT) and chemotherapy with temozolomide (TMZ). Treatment often fails due to the radiation resistance and intrinsic or acquired TMZ resistance of a small percentage of cells with stem cell-like behavior (CSC). The NOTCH signaling pathway is expressed and active in human glioblastoma and NOTCH inhibitors attenuate tumor growth in vivo in xenograft models. Here we show using an image guided micro-CT and precision radiotherapy platform that a combination of the clinically approved $\mathrm{NOTCH} / \gamma$-secretase inhibitor (GSI) RO4929097 with standard of care (TMZ+RT) reduces tumor growth and prolongs survival compared to dual combinations. Our in vitro data demonstrates that GSI in combination with RT and TMZ attenuates proliferation, clonogenic survival and 3D spheroid growth of glioma cell lines U87 and E2 and that inhibits CD133 and SOX2 expression. These findings indicate that NOTCH inhibition combined with standard of care treatment can provide improved survival benefit for GBM and encourage further translational and clinical studies.

\section{Introduction}

Glioblastoma multiforme (GBM) is the most common malignant brain tumor in adults. Multimodal treatment of surgery followed by radiotherapy (RT) and chemotherapy using temozolomide (TMZ) extends the two-year median survival rate of patients from $10 \%$ with radiotherapy alone to $27 \%$ when combined with temozolomide [1]. TMZ acts via alkylation of $\mathrm{O}^{6}$-Guanine, which induces replication errors, DNA damage and cell death. Epigenetic silencing of the O6-methylguanineDNA methyltransferase (MGMT) gene is correlated with an improved survival and response to TMZ [2]. However, approximately $50 \%$ of brain tumors [3, 4] as well as the large majority of the recurrent tumors $[5,6]$ are resistant to TMZ. Tumors often respond to radiotherapy, but subsequent recurrence is almost inevitable due to emergence of radiation resistant cells. Treatment failure leads to a high mortality in GBM patients and therefore, there is a great need for novel treatments that improve clinical management and disease outcome.

In GBM, a subpopulation of radioresistant tumor cells expressing neural stem cell markers such as CD133 with high proliferative and self-renewal capacity have been shown to contribute to tumor recurrence. These cells are often referred to as glioma stem cells [7, 8]. Chemotherapeutic drugs including TMZ as well as radiation therapy 
were shown to predominantly target the CD133-negative population and as such enrich the CD133-positive population [8,9]. Thus, conventional chemoradiotherapy appears to effectively remove the bulk of tumor cells, while leaving many GBM stem cells alive, driving treatment resistance and tumor relapse. Altogether, it appears that eradication of these cells is needed to augment treatment efficacy and outcome.

NOTCH ligands, receptors and target genes are frequently over-expressed in glioma tissues or cell lines [10-12] and the NOTCH pathway plays an important role in maintenance of glioma stem cells [13]. $\gamma$-secretase inhibitors (GSI) are used to inhibit NOTCH pathway in basic and pre-clinical research as well as clinical trials $[14,15]$. Inhibition of NOTCH signaling in glioma stem cells has been shown to impair the tumorigenic capacity of these cells and enhance their radiation and chemo-sensitivity [16-18]. Importantly, GSI administration has also been shown to sensitize the glioblastoma cells that acquired EGF containing fibulin-like extracellular matrix protein 1 (EFEMP1)-mediated TMZ-resistance in vitro and in vivo [19]. While these results are promising, the therapeutic potential of NOTCH inhibition still has to be demonstrated in models that are more representative of the clinical situation to fully assess the benefit in the context of standard of care treatment.

In this study, we investigated for the first time the efficacy of a clinically approved GSI (RO4929097) in tumor control when combined with RT-only, TMZ -only and $\mathrm{RT}+\mathrm{TMZ}$ treatment groups in a $2 \mathrm{D}$ and $3 \mathrm{D}$ spheroid model in vitro as well as in an orthotopic mouse model in vivo. Combining GSI with either RT or TMZ significantly reduced the glioma spheroid growth and tumor progression and prolonged survival when compared with single treatment arms. This effect was most pronounced with the triple combination (GSI+RT+TMZ) and resulted in an increased tumor growth delay when compared with dual treatment arms. Our results suggest that one of the underlying mechanisms for the effect of NOTCH blockade in combination with TMZ and radiation is a reduced clonogenic survival of glioma stem /progenitor cells and that the expression of glioma stem cell marker CD133 was reduced by single or combined treatments with NOTCH inhibitors.

\section{Materials and Methods}

\section{* Cell line culture}

U87MG-Luc2 (U87) and primary E2 glioma cell lines were used in this study. E2 cells were derived from freshly resected human GBM specimens as previously described [20]. U87 cells were cultured in Hyclone MEM/EBSS (Fischer Scientific), $10 \%$ fetal calf serum, $1 \%$ L-Glutamine (Invitrogen), $1 \%$ non-essential amino acids 
(Invitrogen) and 1\% sodium pyruvate (Invitrogen). E2 cells were cultured in MEM $\alpha$ (Gibco) supplemented with 5\% L-glutamine. The U87 cell identity was confirmed using the short tandem repeat (STR) analysis (Identicell, Denmark).

For western blotting and flow cytometry both cell lines were cultured in stem cell enriching conditions in Advanced DMEM F12 medium (Gibco) supplemented with $1 \%$ B27 (Invitrogen), 0.5\% N2 (Invitrogen), $4 \mu \mathrm{g} / \mathrm{ml}$ heparin, $20 \mathrm{ng} / \mathrm{ml}$ fibroblast growth factor (bFGF, Sigma), $20 \mathrm{ng} / \mathrm{ml}$ epidermal growth factor (EGF, Sigma) and $1 \%$ L-Glutamine. Stem cell enriched cultures were grown on Matrigel (BD Biosciences) coated flasks (1:50 dilution) in serum free media.

\section{Orthotopic Brain tumor Implantation and Drug treatment}

Immunocompromised CD1 nu/nu mice were used in this study. Animal work was performed in accordance with national guidelines. The procedure for tumor implantation has been described in detail previously [21]. After confirmation of tumor establishment, GSI was orally administered with a schedule of five days on and two days off for 3 weeks. One-week post-treatment GSI/vehicle mice were irradiated. Directly after irradiation, mice were treated with TMZ/vehicle with a schedule of 3 days on and 4 days off for 2 weeks. The control group received an intraperitoneal (ip) injection of $200 \mu \mathrm{l}$ saline (TMZ-vehicle), and/or oral administration of $200 \mu 1$ of $1.0 \%$ carboxymethyl cellulose with $0.2 \%$ Tween 80 in sterile water (RO4929097-vehicle). The TMZ-only treated group received $30 \mathrm{mg} / \mathrm{kg}$ TMZ (Selleckchem) ip and oral RO4929097-vehicle, while the RO4929097 treated group received $20 \mathrm{mg} / \mathrm{kg}$ RO4929097 orally and TMZ-vehicle ip RT-only group received 8Gy single dose irradiation using the small animal micro-IR (X-RAD 225Cx, Precision X-ray Inc., North Branford, CT). The combined treatment group was administered with the same concentration of TMZ ip, RT and RO4929097 orally. RO4929097 (2,2-dimethyl-N-((S)-6-oxo-6,7-dihydro-5H-dibenzo (b,d) azeptin-7-yl)-N'-(2, 2, 3, 3, 3,-pentafluoro-propyl)-malonamide) has been indicated as GSI in this study. The RO492909 was stored at $4^{\circ} \mathrm{C}$.

\section{In vivo Bioluminescence Imaging (BLI)}

Bioluminescent imaging (BLI) was performed using the Optix MX2 system (ART Inc., Saint-Laurent, QC). Animals were monitored 3x/week. Additionally, a selection of animals in each group were followed by contrast-enhanced micro-CT imaging using the small animal micro-IR (X-RAD 225Cx, Precision X-ray Inc., North 
Branford, CT). All the procedures for imaging and analysis have been extensively described previously [21].

\section{In vivo Irradiation: SmART-Plan.}

Radiotherapy dose was calculated and prescribed using the dedicated small animal radiotherapy planning system SmART-Plan (v1.3.1, Precision X-Ray, North Branford, CT), which has been previously validated [22]. Target delineation, treatment planning and irradiation was performed on anesthetized mice based on a contrast-enhanced micro-CT images that were shown to successfully visualize tumor boundaries as described previously [21,23], and was completed while the animal remained anesthetized on the treatment bed. Within SmART-Plan the tumor was contoured and two 5-mm parallel-opposed beams were placed at the center of the tumor. This beam size ensured complete coverage plus a small margin for set-up uncertainties but still avoided irradiating most of normal brain tissue. Based on earlier work, a homogeneous dose of 8 Gy was prescribed to the target with each beam contributing an equal amount of dose. The source-to-axis distance was $30 \mathrm{~cm}$. Radiation was delivered using the small animal irradiator at $225 \mathrm{kVp}, 12 \mathrm{~mA}$ (filtration of $0.3 \mathrm{~mm}$ of copper) which provides a dose rate of approximately 3 Gy/minute.

\section{D Spheroid Assay}

Spheroid assay was used as a model for treatment response in vitro. 96-well plates were coated with $50 \mu 1$ of autoclaved agarose $(0.750 \mathrm{gr}$ agarose was dissolved in 50 $\mathrm{ml}$ serum free medium) [24]. $2800 \mathrm{U} 87$ cells/200 $\mu$ l complete medium were seeded on the agarose coated plates per well. One spheroid per well was formed within 4 days after seeding. For each condition, at least 12 spheroids were tested. 4 days postseeding first phase-contrast pictures were taken from all the spheroids individually and then the sphere volume was analyzed using an in-housed script using MATLAB program (MathWorks, 2009b, CT) and available for download [25]. Directly after image acquisition, treatment regimens were administered. GSI (RO4929097) and TMZ were administered immediately after irradiation in RT treated groups. The control group received only DMSO. Final concentration for GSI-only and TMZ-only treated group was $10 \mu \mathrm{M}$ and $5 \mu \mathrm{M}$, respectively. The RT-only group was irradiated with a single dose of $4 \mathrm{~Gy}$. All the combination groups were treated with the same doses. So as not to disrupt the sphere structure, in all treatment groups $100 \mu 1$ of 
medium was discarded very carefully and $100 \mu$ of the $2 \mathrm{x}$ treatment pre-mixtures were added. The drugs were washed out by refreshing the medium 3x/week. TMZ was washed out after three days incubation in the first week and the same treatment procedure was repeated in the second week, while GSI was refreshed twice weekly for two weeks. TMZ was diluted in DMSO and stored in $100 \mathrm{mM}$ stock at $-80^{\circ}$. GSI was diluted in DMSO and stored in $10 \mathrm{mM}$ stock at $-20^{\circ}$. Sphere volumes were measured 3 times a week and experiments were terminated when treated spheres reached $20 \mathrm{x}$ treatment volume.

\section{* Proliferation assay}

500 cells/well (U87) or 2000 cells/well (E2) were seeded in 96 well plates and were treated $24 \mathrm{~h}$ post-seeding with $5 \mu \mathrm{M}$ TMZ and/or 10 $\mu \mathrm{M}$ GSI (RO4929097) and/or single dose of 4Gy RT (Philips X-ray tube; $225 \mathrm{kV} ; 10 \mathrm{~mA}$ ). The three treatments were tested as single, double and triple combinations with 6 replicates per condition. $72 \mathrm{~h}$ post-treatment TMZ was washed out and GSI was refreshed. Cell confluency was monitored every $2 \mathrm{~h}$ using the phase-contrast mode of the IncuCyte TM FLR (2011A) live-imager. TMZ was diluted in DMSO and stored in $100 \mathrm{mM}$ stock at $80^{\circ}$. GSI was diluted in DMSO and stored in $10 \mathrm{mM}$ stock at $-20^{\circ}$.

\section{Quantitative Real Time PCR (qPCR)}

RNA extraction was performed using the NucleoSpin RNA II kit (Bioke). cDNA was prepared using the iScript cDNA Synthesis kit (BioRad). Reactions were carried out in a $10 \mu 1$ volume using sensiMix SYBR low-ROX kit (GC Biotech) with the ABI Prism 7500 Sequence Detection System. Values for each gene were normalized to expression levels of Actin RNA. Primer sequences have been described previously [26].

\section{Clonogenic survival assay}

Cells were seeded in 6-cm dishes to be $70 \%$ confluent at the time of irradiation. Cells were irradiated with $2 \mathrm{~Gy}, 4 \mathrm{~Gy}$ or $6 \mathrm{~Gy}$ and non-treated (Philips X-ray tube; $225 \mathrm{kV}$; $10 \mathrm{~mA})$. Directly after irradiation, cells were trypsinized and seeded at densities of 250 or 1000 cells per well for E2 and 500 cells per well for U87 in triplicate in 6well plates and allowed to adhere for $4 \mathrm{~h}$ in complete medium. Then, the medium was replaced with treated medium: DMSO in control group, $10 \mu \mathrm{M}$ TMZ for E2 cells and 
$5 \mu \mathrm{M}$ TMZ for U87 cells for 48h in TMZ-only treated group and 10 $\mu \mathrm{M}$ RO4929097 in GSI-only treated group. The combination groups were also treated with the same concentrations. RO4929097 was refreshed every 5 days during the experiment. Colonies were counted manually after 2 weeks. The minimum number of cells per counted colony was 30 .

\section{Flow cytometry}

Cells were first irradiated with 4Gy and then treated with $10 \mu \mathrm{M}$ RO4929097 and $10 \mu \mathrm{M}$ TMZ as well as their combination. Cultures were disaggregated with accutase 4 days post-treatment, cells were washed with PBS once and incubated with CD133/2-PE (1:200; Miltenyi Biotech) or isotype control antibody on ice for $30 \mathrm{~min}$. Then cells were washed with PBS $2 x$, resuspended in $200 \mu 1$ PBS and fixed in $1 \%$ paraformaldehyde. Just before measurements of each FACS tube DAPI (final concentration $3 \mu \mathrm{M}$ ) was resuspended in each tube. FACS analysis was carried out on FACS Calibur machine (BD Biosciences).

\section{* Western blotting}

Cells were first irradiated with 4Gy and then treated with $10 \mu \mathrm{M}$ RO4929097, 10 $\mu \mathrm{M}$ TMZ as well as their combination. 4 days post-treatment total cell lysate was prepared using SDS lysis buffer. Lysates were blotted onto a PVDF-membrane. Membranes were probed overnight at $4^{\circ} \mathrm{C}$ with primary antibodies and bound antibodies were visualized using HRP-linked secondary antibodies (Cell-Signaling) and ECL Luminescence (Pierce Biotechnology). Anti-SOX2 (ab75485, Abcam), anti-Nestin (ab6320, Abcam), anti-beta-tubulin III (T3952, Sigma-Aldrich), antiGFAP (ab5804, Millipore) and anti-Lamin A/C (Sigma-Aldrich) were used at 1:1000 dilution.

\section{* Statistical analysis}

Statistical analysis was performed using GraphPad Prism Software (v5.02, San Diego, CA). For all measured quantities mean \pm SEM are reported except where stated. Mann-Whitney or ANOVA tests were used to analyze differences in growth delay and in clonogenic survival. The log-rank (Mantel-Cox) test was used to compare the survival curves. A p-value smaller than 0.05 was considered statistically significant. 


\section{Results}

\section{NOTCH pathway is active in GBM cells}

To address whether the NOTCH pathway was active in our glioma cell lines, we analyzed the gene expression profile of NOTCH receptors (NOTCH1-4), ligands (DLL 1,3,4 JAG 1,2) and target (HES, HEY) genes. Differential expression of NOTCH pathway components was confirmed by qPCR in both U87 and primary E2 GBM cells (Figure $1 \mathrm{~A}$ and C). Blocking NOTCH$/ \gamma$-secretase using 5 or $10 \mu \mathrm{M}$ of GSI (RO4929097) significantly reduced the expression of the NOTCH target genes HES1 and HEY2 in U87 cells (Figure1B) and HEY1 and HEY2 in E2 cells (Figure 1D). HEY2 expression in E2 cells was only significantly reduced at the $10 \mu \mathrm{M}$ GSI.

\section{NOTCH inhibition in combination with RT and TMZ attenuates proliferation and clonogenic survival in vitro}

Next, we investigated the effect of NOTCH inhibition as monotherapy or in combination with RT and TMZ on proliferation of U87 and E2 cells in a 2D growth format. In U87 cells, treatment with GSI, TMZ and TMZ+GSI did not affect proliferation significantly compared with the vehicle control (DMSO). Upon RT (4Gy) treatment, GSI-only, TMZ-only and TMZ+GSI significantly reduced proliferation compared to vehicle control ( $\mathrm{p}<0.001$, in all cases) in U87 cells (Figure 2A). In E2 cells treatment with GSI and TMZ alone did not affect proliferation significantly compared with the vehicle control (DMSO), while TMZ+GSI did $(\mathrm{p}<0.05)$. Upon RT (4Gy) treatment, GSI significantly reduced proliferation compared to vehicle control $(\mathrm{P}<0.01)$. Similarly, after RT treatment TMZ+GSI significantly reduced proliferation compared to vehicle control, TMZ and GSI treatments $(\mathrm{p}<0.0001, \mathrm{p}<0.001$ and $\mathrm{p}<0.0001$, respectively) (Figure $2 \mathrm{~B}$ ).

Next, we examined the effect of combination treatments on clonogenic survival. In U87 cells, upon RT, GSI and TMZ did not affect the clonogenic survival compared with the vehicle control (DMSO), however the survival fraction was markedly reduced in GSI+TMZ treatment group at 2, 4, and 6Gy ( $<<0.0001$ ) (Figure 2C). The mean inactivation dose (50\% reduction in clonogenicity) for GSI $+\mathrm{TMZ}$ was $2.28 \mathrm{~Gy}$ and for control +TMZ 3.2Gy, respectively. In E2 cells, RT, GSI and TMZ reduced the clonogenic survival compared with the vehicle control (DMSO) $(\mathrm{p}=0.047$ and $\mathrm{p}=0.02$, respectively). Clonogenicity was further reduced in GSI $+\mathrm{TMZ}$ irradiated 
cells $(\mathrm{p}=0.0002)$ (Figure 2D). A representative image of the survived colony in E2 cells upon different treatment combinations is shown (Supplementary Figure 1).

We then investigated the effects of single and combined treatment in a threedimensional spheroid assay of U87 GBM cells. Four days post-seeding, U87 spheroids were irradiated with a single dose RT (4Gy) and then treated with TMZ $(5 \mu \mathrm{M})$ and GSI $(10 \mu \mathrm{M})$ (Figure $3 \mathrm{~A})$.

In the absence of radiation (0Gy), NOTCH inhibitor alone did not affect the spheroid growth compared with the vehicle control, however when combined with TMZ an enhanced effect was observed $(\mathrm{p}=0.01)$ (Figure 3B). RT treatment significantly delayed spheroid growth, which was further enhanced upon treatment with GSI compared to non-RT treated cells $(\mathrm{p}=0.005$ and $\mathrm{p}=0.007$, respectively) (Figure 3C). Finally, GSI combined with TMZ and single dose RT treatment resulted in the most pronounced growth delay when compared with either treatment combination including the standard of care, TMZ+RT, $(\mathrm{p}=0.02)$ (Figure 3D-E). Representative images of spheroids in each treatment group at different time points post-treatment are shown (Supplementary Figure 2). For E2 cells, spheroids formed but were not able to grow consistently over time in agarose-coated plates (data not shown).

\section{NOTCH inhibition combined with standard of care treatment reduces tumor growth and prolongs survival of intracranial GBM in vivo}

Next, we assessed the effects of NOTCH inhibition in combination with TMZ and single dose RT on the survival of mice with U87 intracranial glioblastoma. We previously determined that single dose RT of $8 \mathrm{~Gy}$ results in a significant but modest tumor growth delay compared to tumors that did not receive any irradiation [23], thereby providing a window of opportunity where the combined effects of RT and TMZ could be measured. One-week post-implantation, mice were imaged with Bioluminescence imaging (BLI) $3 \mathrm{x} /$ week. Contrast-enhanced micro-CT was also used to monitor tumor growth periodically as reported previously [23]. Upon confirmation of tumor growth by BLI or micro-CT, mice were randomized into eight different treatment arms. These mice were treated with GSI/vehicle, TMZ/vehicle as well as radiation (Figure 5A) using a dedicated small animal irradiator and associated treatment planning software (SmART-Plan) to create irradiation plans to deliver a conformal dose of $8 \mathrm{~Gy}$ to the tumor with minimal normal tissue exposure. An example of the parallel-opposed radiation treatment in sagittal, axial and coronal planes (Figure 4A-C) and the dose volume histograms (DVHs) for all irradiated mice is shown (Figure 4D). These DVHs demonstrate that a highly uniform dose 
distribution across the tumor volume is achieved at the desired prescription dose of 8 Gy as previously determined [23].

We determined the tumor growth delay using bioluminescence by calculating the time it takes for each tumor to reach 10x BLI signaling intensity from start of treatment (T10xSI). A significant growth delay was observed in GSI-, 8Gy- and TMZ-only treated groups (22 days, 25 days, and 36.4 days, respectively) compared to the control group ( 16.1 days) ( $\mathrm{p}<0.05$ for GSI- and 8 Gy-only groups, $\mathrm{p}<0.001$ for TMZ-only group) (Figure 5B). The median survival defined based on the neurological signs and abnormal behavior as humane endpoints for the GSI- and $8 \mathrm{~Gy}$ - single treatments were not significantly different from the control $( \pm 20.5$ days for GSI-only, \pm 20 days for 8Gy-only, \pm 19 days for control) (Figure 5C). Addition of GSI to either TMZ or $8 \mathrm{~Gy}$ irradiation resulted in a significant growth delay (46 days and 28.3 days, respectively) when compared with 8 Gy or TMZ-only groups ( $\mathrm{p}<0.01$ and $\mathrm{p}<0.001$, respectively) (Figure 5B). The median survival for these groups was significantly prolonged compared to control ( \pm 33days for GSI+8Gy and \pm 44 days for GSI+TMZ, $\mathrm{p}<0.001$ for both groups) (Figure 5C). Similarly, the standard of care treatment (8Gy+TMZ) resulted in a significant growth delay $( \pm 58$ days) when compared with 8 Gy- or TMZ-only groups $(\mathrm{p}<0.0001)$ as well as the TMZ+GSI group $(\mathrm{p}<0.05)$. The median survival for this group was significantly prolonged compared to single treatments $( \pm 55$ for $8 \mathrm{~Gy}+\mathrm{TMZ}, \mathrm{p}<0.001$ comparing with $8 \mathrm{~Gy}$ - and TMZ-only). The most profound growth delay and increase in median survival was observed in mice treated with triple combination of 8Gy, TMZ and GSI ( \pm 81 days for 3 out of 4 mice, $p<0.001$ compared with control and GSI $+8 G y, p<0.05$ compared with GSI+TMZ and 8GY+TMZ) (Figure 5C). One out of 4 mice showed a cure defined as being tumor-free for $>14$ weeks post start of treatment. Representative BLI and contrast-enhanced micro-CT images of a mouse in each treatment group at different time points post-treatment are shown (Supplementary Figure 3). We observed significant down regulation of HES1 mRNA expression in tumors from GSI treated mice, compared to control treated mice $(\mathrm{p}=0.01)$ (Figure 5D). HEY2 mRNA was not altered upon GSI treatment (data not shown).

No dose limiting toxicities were observed for single and double treatments although weight loss $<20 \%$ was invariably observed within the first or second week after treatment. Importantly, four out of eight mice receiving triple-combination treatments were euthanized due to a significant weight loss prior to reaching endpoint despite very small histologically confirmed tumor size. While we do not know the exact reason for this, we speculate that this could be due to toxicity associated with the triple therapy combination. 


\section{Combination treatment $(G S I+T M Z+R T)$ reduces the stem cell population}

To investigate the mechanism of increased radio- and chemo-sensitivity upon NOTCH inhibition, we evaluated the expression of the glioma stem cell marker CD133 using flow cytometry 4 days post-treatment in U87 and E2 cells grown under adherent stem cell conditions (Figure 6A-B). We observed a 4-fold increase in CD133 expression in primary E2 and U87 cells upon single dose 4Gy RT. Pretreatment with either GSI or TMZ reduced CD133 expression by 2.1 and 2.9-fold in primary E2 cells as well as 1.8 and 2-fold in U87 cells, respectively (Figure 6A-B). No significant differences were observed in TMZ + GSI treated cells (Supplementary Figure 4). Under the same treatment condition, SOX2 mRNA expression was significantly reduced upon GSI+RT and GSI+TMZ+RT treatments in both E2 and U87 (Supplementary Figure 4A-B). A strong reduction in SOX2 protein expression in E2 cells was only observed in the triple combination (Supplementary Figure 4C). GSI strongly inhibited NOTCH cleavage as shown by immunoblotting with Val1744 recognizing the active cleaved form of NOTCH1 (NICD1) (Supplementary Figure $4 C)$.

\section{Discussion}

Given the highly proliferative and infiltrative nature of GBM and the lack of curative treatment, novel treatments are urgently needed in relevant in vitro and preclinical models that mimic clinical routine to enable swift translation. In this study, we show the therapeutic benefit of the addition of the clinically approved $\mathrm{NOTCH} / \gamma$ secretase inhibitor (RO4929097) combined with the standard of care in GBM patients that undergo radiotherapy and chemotherapy. Our results show that in an orthotopic model for glioblastoma, treatment with NOTCH inhibition enhances radiotherapy, TMZ chemotherapy as well as a combination of both such that in 1 out of 4 mice tumor cure (defined as being tumor-free for $>14$ weeks) was achieved and in the remaining cohort a survival benefit of $\sim 62$ days and $\sim 26$ days was obtained when compared to the untreated mice or the standard of care treatment group (TMZ+RT), respectively.

Previously, we have shown the rational for using the 3D spheroid model system to better predict clinical efficacy [23]. Consistently, in this study we have shown that in vitro screening of the NOTCH inhibition combined with chemo-radiation (GSI+TMZ+RT) using 3D spheroid system is a robust methodology to identify 
combination therapies for GBM that reflect in vivo response. The 3D model used here however still lack important factors from the tumor micro-environment such as vasculature, fibroblasts or immune cells. Therefore, we used our previously established orthotopic GBM model [21] to address the in vivo efficacy of the NOTCH inhibitors combined with chemoradiation. The effect of radiation on normal brain tissue is a determining factor in treatment of CNS tumors because of the radiation-induced necrosis. Considering this and in contrast to standard devices using a single large beam, we used an image-guided small animal micro-irradiator device that combines micro-CT imaging with conformal irradiation and millimeteradjustable beams [23]. This was combined with the use of the treatment planning software (SmART-Plan) and made radiation treatment planning and delivery highly accurate in this orthotopic GBM model. Similar to that in clinical practice, we determined dose-volume histograms (DVH) showing that the X-ray beams were highly conformal and localized to the tumor region, minimizing exposure to organs at risk such as the normal brain [22, 23].

Based on our findings, growth responses between the in vitro spheroid and in vivo preclinical models did complement one another; however, there were some discrepancies as well. For example, NOTCH inhibition alone did not affect the spheroid growth in U87 cells but did result in a significant growth delay in vivo according to the BLI data. Our data are in accordance with the observation in other studies that showed $\gamma$-secretase inhibitors alone do not affect neurosphere formation in vitro [17], but single agents activity of these inhibitors prolong survival in orthotopic glioblastoma xenografts [18]. One explanation could be the contribution of Notch inhibition in the tumor microenvironment for example by disrupting tumor angiogenesis [27] or blocking endothelial to tumor cell signaling $[28,29]$ which was not addressed in this study. We additionally explored combination treatments of GSI with RT or TMZ as mono-therapy. In our 3D spheroid model and GBM tumor bearing mice, NOTCH inhibition potentiated the effect of RT or TMZ. Our findings confirmed previous results that showed the enhanced therapeutic effect of combined GSI with TMZ (in vitro and ex vivo) or GSI with RT (in vitro and in vivo) compared to each treatment alone [17, 28, 30]. While these findings are important to move NOTCH inhibitors forward as GBM therapeutics, contribution of GSI to current standard of care treatment (TMZ+RT) has never been reported to the best of our knowledge. In this study, for the first time we show that NOTCH inhibition in combination with TMZ+RT significantly enhances the 3D spheroid growth delay in vitro and prolongs survival of the mice bearing the orthotopic glioma compared to TMZ+RT. This result is encouraging in developing new combination therapy for GBM; however, the clinical relevance of this study could further be maximized by 
the use of patient derived xenografts that may better reveal the gene expression profiles of the glioma patients and typical histological characteristics (microinfiltrative, highly vascularized, palisading necrosis) compared to established glioma cell lines [31]. Recently, integrated genomic profiling of glioblastoma has provided an improved molecular understanding of the etiology and has led to new classifications with prognostic and predictive importance [32-35]. Targeted therapeutics against 'actionable' targets have however led to limited clinical efficacy exemplified by Avastin and EGFR inhibitor trials in GBM [36]. This may in part be due to high heterogeneity in GBM and clonal expansion driven by treatment or de novo acquired resistance mechanisms [37]. Therapeutics targeting of the survival of tumor initiating cells are more likely to be successful as they target "driver" populations. Identification of these driver populations needs further investigation but our work and that of others highlight the importance of CD133+ cells in growth and treatment response and their dependence on $\mathrm{NOTCH}$ signaling. While $\mathrm{NOTCH}$ inhibitors may be effective in proneural subtype which has high NOTCH activity [38], in low grade glioma's NOTCH1 loss of function mutations are observed and NOTCH inhibitors may be less evident [32]. While it remains possible that activating mutations in NOTCH1 (or NOTCH2/3) are only present in a subset of the tumor cells, it emphasizes the need for in depth molecular analysis prior to patient selection for NOTCH inhibitor therapy.

To elucidate the underlying mechanism of the treatment response in our study, we observed that NOTCH inhibition influenced the radiation-enhanced CD133 expression, in line with the expected role of NOTCH in the maintenance of glioma stem cells [28]. Importantly, it has been reported that endothelial cells can function as a stem cell niche to promote $\mathrm{CD} 133+$ self-renewal in glioblastoma [29] by providing NOTCH ligands that activate $\mathrm{NOTCH}$ receptors in $\mathrm{CD} 133+$ cells. Targeting NOTCH ligands in endothelial cells reduced the CD133+ population as well as the growth of intracranial glioma xenografts [39]. In line with this, Hovinga et al. showed that NOTCH inhibition in glioma explants resulted in decreased selfrenewal of CD133+ cells, which correlated with a decrease in endothelial cells [28]. If NOTCH inhibition also reduced CD133+ sub-populations directly or indirectly via endothelial cell signaling in vivo was not addressed in our study and requires further investigation.

Our data showed that temozolomide was equally effective as single NOTCH inhibitor treatment or dual treatment in reducing radiation increase of CD133+ cells, consistent with other reports [40]. This finding could only be partly replicated in our clonogenics assays (not enriched for CD133+) suggesting that while CD133+ marks clonogenic cells, it does not reflect only glioma stem cells. Others report that CD133+ 
cells are resistant to chemotherapy $[9,41]$. However, in some of these studies the viability of $\mathrm{CD} 133^{+}$cells was assessed only in short-term culture using extremely high TMZ concentrations $(0.2-2 \mathrm{mM})$ and culture conditions not suited for the maintenance of the cancer stem cell phenotype $[42,43]$. In line with our data, TMZ treatment $(5-25 \mu \mathrm{M})$ on glioma cells grown under stem cell enriched conditions reduced proliferation and survival at very low TMZ concentrations [44] and inhibited the growth of neurosphere in $\mathrm{CD} 133^{+}$sub-population and reduced tumorigenicity $[40,45]$. Further investigation is needed to reconcile these findings but suggest that within the $\mathrm{CD} 133+$ population a treatment resistant clonogenic population exist sensitive to NOTCH inhibition which support this study and other studies showing that NOTCH inhibitors can sensitize to TMZ in xenografts [17]

Although our data show depletion of glioma cancer stem cells upon GSI and TMZ administration after radiotherapy, the exact mechanism for the enhanced efficacy of the triple combination (GSI+RT+TMZ) treatment in vivo is not known. For example, triple treatment did not further reduce $\mathrm{CD} 133+$ surface population compared to dual treatments. We did observe a reduction of the glioma stem cell marker SOX2 in the triple combination only. Silencing of SOX2 in glioma tumor initiating cells blocks proliferation and suppresses tumorigenicity [46]. To what extent the expression of SOX2 and CD133 overlap in our systems is not known and the phenotypic identification of the cell types affected by these treatments in vivo would be a key step [47].

Various clinical trials are underway to investigate whether NOTCH blockade using $\gamma$-secretase inhibitors improves cancer treatment [48]. However, one of the major challenges on the way is the untoward side effects associated with NOTCH inhibitors, especially the cytotoxicity in the gastrointestinal tract [49]. Most of the available $\gamma$-secretase inhibitors are originally engineered for treatment of Alzheimer's diseases and therefore are designed for long-term and systemic treatments and are able to pass the blood brain barrier. New NOTCH inhibitors for cancer treatment are specifically designed not to cross the blood brain barrier because of cognitive defects seen in AD patients related to long-term on target NOCTH inhibition [50]. It will be crucial therefore to monitor GBM patients treated with NOTCH inhibitors for these side effects as well. To maximize the therapeutic effects and minimize the systemic NOTCH-related side effects, improved dosage regimens have now been reported in phase 1 clinical trials that "spare " the intestine [51]. In our study, using the same orally administrated GSI RO4929097 efficacy was observed with similar scheduling orally for (5 days on and 2 days off for 3 weeks), which did not lead to any weight loss or other toxic effects. However, we did observe deaths not due to the tumor burden but rather due to toxicity associated with the 
prescribed triple therapy regimen by a yet unknown mechanism. Therefore, balancing efficacy and toxicity of $\gamma$-secretase inhibitors by optimizing treatment cycles and dosing schemes is paramount for successful future clinical applications. The dose of RO4929097 inhibitor used in our study is in a therapeutically relevant pharmacokinetic range for humans [52]. In this regard, a current clinical trial is recruiting patients to investigate the efficacy of RO4929097 in combination with radiation and TMZ (http:// ClinicalTrials.gov identifier NCT01119599). Taken together, we believe that our findings are promising for clinical translation to increase survival in GBM patients but that unanticipated toxicities may occur. Therefore, identification of a subset of patients who have active NOTCH signaling could predict the likelihood of increased response to anti-NOTCH therapy. More studies are needed to fully exploit the potent NOTCH inhibitors but also to recognize potential aggravating conditions that may occur.

\section{Acknowledgements}

The NOTCH inhibitor RO4929097 was generously provided by Roche Laboratories and the National Cancer Institute (NIH). Funding was provided in part by ERC grant 607061 .

\section{Conflict of interest}

The authors have declared that no competing interests exist. 


\section{References}

1. Stupp, R., et al., Radiotherapy plus concomitant and adjuvant temozolomide for glioblastoma. N Engl J Med, 2005. 352(10): p. 987-96.

2. Minniti, G., et al., Correlation between O6-methylguanine-DNA methyltransferase and survival in elderly patients with glioblastoma treated with radiotherapy plus concomitant and adjuvant temozolomide. $\mathrm{J}$ Neurooncol, 2011. 102(2): p. 311-6.

3. Friedman, H.S., et al., DNA mismatch repair and O6-alkylguanine-DNA alkyltransferase analysis and response to Temodal in newly diagnosed malignant glioma. Journal of Clinical Oncology, 1998. 16(12): p. 3851-7.

4. Hegi, M.E., et al., Correlation of O6-methylguanine methyltransferase (MGMT) promoter methylation with clinical outcomes in glioblastoma and clinical strategies to modulate MGMT activity. Journal of Clinical Oncology, 2008. 26(25): p. 4189-99.

5. Cahill, D.P., et al., Loss of the mismatch repair protein MSH6 in human glioblastomas is associated with tumor progression during temozolomide treatment. Clinical Cancer Research, 2007. 13(7): p. 2038-2045.

6. Yip, S., et al., MSH6 Mutations Arise in Glioblastomas during Temozolomide Therapy and Mediate Temozolomide Resistance (vol 15, pg 4622, 2009). Clinical Cancer Research, 2013. 19(16): p. 4543-4544.

7. Mannino, M. and A.J. Chalmers, Radioresistance of glioma stem cells: intrinsic characteristic or property of the 'microenvironment-stem cell unit'? Mol Oncol, 2011. 5(4): p. 374-86.

8. Bao, S., et al., Glioma stem cells promote radioresistance by preferential activation of the DNA damage response. Nature, 2006. 444(7120): p. 75660.

9. Liu, G., et al., Analysis of gene expression and chemoresistance of CD133+ cancer stem cells in glioblastoma. Molecular cancer, 2006. 5: p. 67.

10. Teodorczyk, M. and M.H. Schmidt, Notching on Cancer's Door: Notch Signaling in Brain Tumors. Front Oncol, 2014. 4: p. 341.

11. Purow, B.W., et al., Expression of Notch-1 and its ligands, Delta-like-1 and Jagged-1, is critical for glioma cell survival and proliferation. Cancer Res, 2005. 65(6): p. 2353-63.

12. Kanamori, M., et al., Contribution of Notch signaling activation to human glioblastoma multiforme. J Neurosurg, 2007. 106(3): p. 417-27.

13. Wang, Z., et al., Emerging role of Notch in stem cells and cancer. Cancer Lett, 2009. 279(1): p. 8-12.

14. Takebe, N., et al., Targeting Notch, Hedgehog, and Wnt pathways in cancer stem cells: clinical update. Nat Rev Clin Oncol, 2015. 12(8): p. 445-64.

15. Rizzo, P., et al., Rational targeting of Notch signaling in cancer. Oncogene, 2008. 27(38): p. 5124-31. 
16. Fan, X., et al., NOTCH pathway blockade depletes CD133-positive glioblastoma cells and inhibits growth of tumor neurospheres and xenografts. Stem Cells, 2010. 28(1): p. 5-16.

17. Gilbert, C.A., et al., Gamma-secretase inhibitors enhance temozolomide treatment of human gliomas by inhibiting neurosphere repopulation and xenograft recurrence. Cancer Res, 2010. 70(17): p. 6870-9.

18. Chu, Q., et al., Prolonged inhibition of glioblastoma xenograft initiation and clonogenic growth following in vivo Notch blockade. Clin Cancer Res, 2013. 19(12): p. 3224-33.

19. Hiddingh, L., et al., EFEMP1 induces gamma-secretase/Notch-mediated temozolomide resistance in glioblastoma. Oncotarget, 2014. 5(2): p. 363-74.

20. Al-Mayhani, T.M.F., et al., An efficient method for derivation and propagation of glioblastoma cell lines that conserves the molecular profile of their original tumours. Journal of Neuroscience Methods, 2009. 176(2): p. 192-199.

21. Yahyanejad, S., et al., Complementary Use of Bioluminescence Imaging and Contrast-Enhanced Micro-Computed Tomography in an Orthotopic Brain Tumor Model. Molecular Imaging, 2014. 13: p. 1-8.

22. van Hoof, S.J., P.V. Granton, and F. Verhaegen, Development and validation of a treatment planning system for small animal radiotherapy: SmART-Plan. Radiother Oncol, 2013. 109(3): p. 361-6.

23. Yahyanejad, S., et al., An image guided small animal radiation therapy platform (SmART) to monitor glioblastoma progression and therapy response. Radiother Oncol, 2015.

24. Friedrich, J., et al., Spheroid-based drug screen: considerations and practical approach. Nat Protoc, 2009. 4(3): p. 309-24.

25. Granton, P. Sphere assay image size analysis. 2015; Available from: https://www.mathworks.com/matlabcentral/fileexchange/54312-sphereassay-image-size-analysis

26. Theys, J., et al., High NOTCH activity induces radiation resistance in non small cell lung cancer. Radiother Oncol, 2013. 108(3): p. 440-5.

27. Ridgway, J., et al., Inhibition of Dll4 signalling inhibits tumour growth by deregulating angiogenesis. Nature, 2006. 444(7122): p. 1083-7.

28. Hovinga, K.E., et al., Inhibition of notch signaling in glioblastoma targets cancer stem cells via an endothelial cell intermediate. Stem Cells, 2010. 28(6): p. 1019-29.

29. Calabrese, C., et al., A perivascular niche for brain tumor stem cells. Cancer Cell, 2007. 11(1): p. 69-82.

30. Dantas-Barbosa, C., et al., Inhibition of the NOTCH pathway using gammasecretase inhibitor RO4929097 has limited antitumor activity in established glial tumors. Anticancer Drugs, 2014.

31. Li, A.G., et al., Genomic changes and gene expression profiles reveal that established glioma cell lines are poorly representative of primary human gliomas. Molecular Cancer Research, 2008. 6(1): p. 21-30. 
32. Cancer Genome Atlas Research, N., et al., Comprehensive, Integrative Genomic Analysis of Diffuse Lower-Grade Gliomas. N Engl J Med, 2015. 372(26): p. 2481-98.

33. Frattini, V., et al., The integrated landscape of driver genomic alterations in glioblastoma. Nat Genet, 2013. 45(10): p. 1141-9.

34. Parsons, D.W., et al., An integrated genomic analysis of human glioblastoma multiforme. Science, 2008. 321(5897): p. 1807-1812.

35. Brennan, C.W., et al., The Somatic Genomic Landscape of Glioblastoma. Cell, 2013. 155(2): p. 462-477.

36. Prados, M.D., et al., Toward precision medicine in glioblastoma: the promise and the challenges. Neuro Oncol, 2015. 17(8): p. 1051-63.

37. Snuderl, M., et al., Mosaic amplification of multiple receptor tyrosine kinase genes in glioblastoma. Cancer Cell, 2011. 20(6): p. 810-7.

38. Saito, N., et al., A high Notch pathway activation predicts response to gamma secretase inhibitors in proneural subtype of glioma tumor-initiating cells. Stem Cells, 2014. 32(1): p. 301-12.

39. Zhu, T.S., et al., Endothelial Cells Create a Stem Cell Niche in Glioblastoma by Providing NOTCH Ligands That Nurture Self-Renewal of Cancer StemLike Cells. Cancer Research, 2011. 71(18): p. 6061-6072.

40. Beier, D., et al., Temozolomide preferentially depletes cancer stem cells in glioblastoma. Cancer Res, 2008. 68(14): p. 5706-15.

41. Kang, M.K. and S.K. Kang, Tumorigenesis of chemotherapeutic drugresistant cancer stem-like cells in brain glioma. Stem Cells Dev, 2007. 16(5): p. 837-47.

42. Lee, J., et al., Tumor stem cells derived from glioblastomas cultured in bFGF and EGF more closely mirror the phenotype and genotype of primary tumors than do serum-cultured cell lines. Cancer Cell, 2006. 9(5): p. 391-403.

43. Li, A., et al., Genomic changes and gene expression profiles reveal that established glioma cell lines are poorly representative of primary human gliomas. Mol Cancer Res, 2008. 6(1): p. 21-30.

44. Clement, V., et al., HEDGEHOG-GLI1 signaling regulates human glioma growth, cancer stem cell self-renewal, and tumorigenicity. Current biology : CB, 2007. 17(2): p. 165-72.

45. Blough, M.D., et al., Sensitivity to temozolomide in brain tumor initiating cells. Neuro Oncol, 2010. 12(7): p. 756-60.

46. Gangemi, R.M., et al., SOX2 silencing in glioblastoma tumor-initiating cells causes stop of proliferation and loss of tumorigenicity. Stem Cells, 2009. 27(1): p. 40-8.

47. Lemke, D., et al., Primary glioblastoma cultures: can profiling of stem cell markers predict radiotherapy sensitivity? J Neurochem, 2014.

48. Andersson, E.R. and U. Lendahl, Therapeutic modulation of Notch signalling - are we there yet? Nature Reviews Drug Discovery, 2014. 13(5): p. 359-380. 
49. Milano, J., et al., Modulation of notch processing by gamma-secretase inhibitors causes intestinal goblet cell metaplasia and induction of genes known to specify gut secretory lineage differentiation. Toxicol Sci, 2004. 82(1): p. 341-58.

50. Doody, R.S., et al., A phase 3 trial of semagacestat for treatment of Alzheimer's disease. N Engl J Med, 2013. 369(4): p. 341-50.

51. Tolcher, A.W., et al., Phase I study of RO4929097, a gamma secretase inhibitor of Notch signaling, in patients with refractory metastatic or locally advanced solid tumors., in Journal of Clinical Oncology. 2012, American Society of Clinical Oncology. p. 2348-2353.

52. Luistro, L., et al., Preclinical profile of a potent gamma-secretase inhibitor targeting notch signaling with in vivo efficacy and pharmacodynamic properties. Cancer Res, 2009. 69(19): p. 7672-80. 


\section{Figures and Tables}
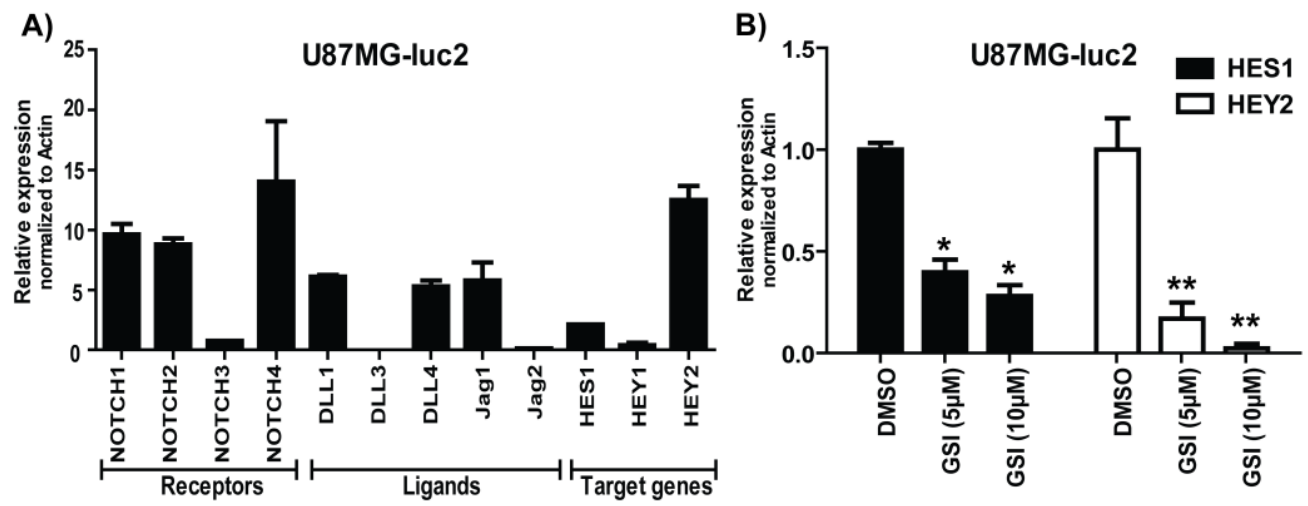

C)

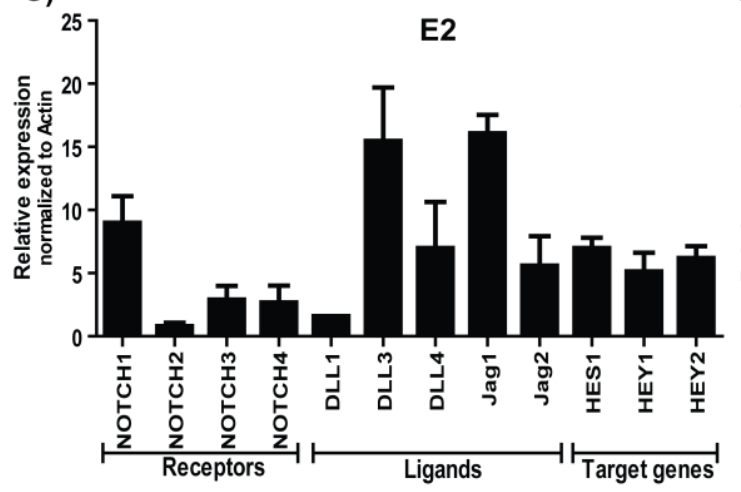

D)

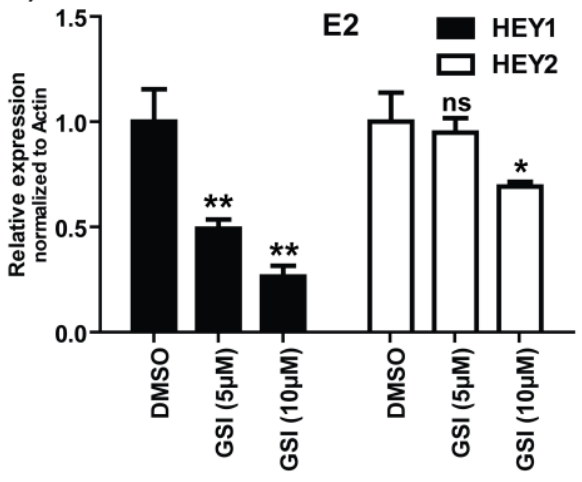

Figure 1: NOTCH signaling in GBM cell lines. A and C) mRNA expression of the NOTCH receptors, ligands and target genes in GBM cell lines were determined by qRT-PCR in U87MG-Luc2 and E2 cells. B and D) mRNA expression of NOTCH target genes (HES1, HEY1 and HEY2) reduced after treatment with different concentrations of clinically available GSI RO4929097 as determined by qRT-PCR. Values were normalized to actin. Error bars indicate SEM, $\left({ }^{*} \mathrm{P}<0.05\right.$, $* \mathrm{P}<0.01$, ns: not significant). 

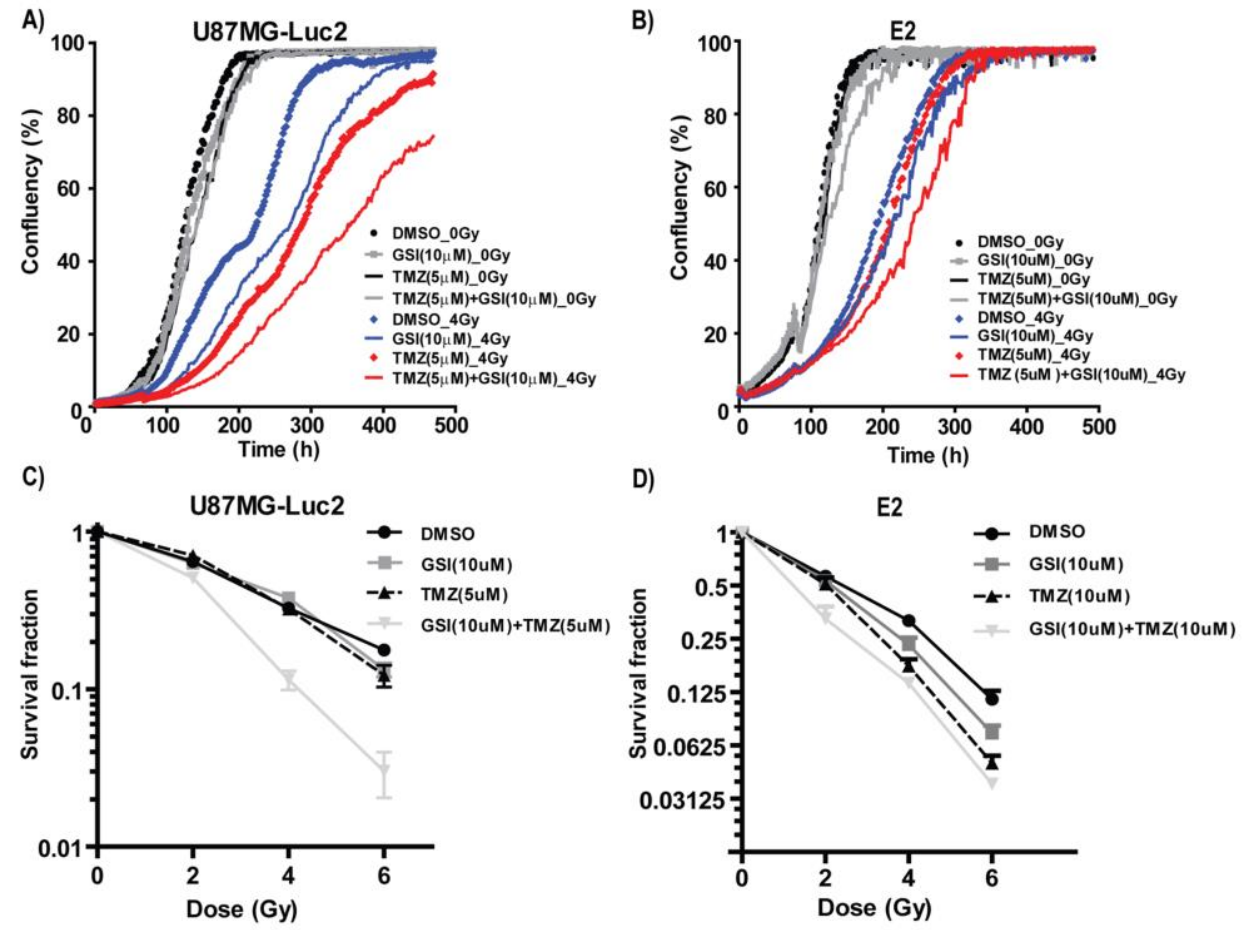

Figure 2: Effect of NOTCH inhibition combined with TMZ and RT on proliferation and clonogenicity in vitro. A-B) Proliferation analysis of U87MGLuc2 and E2 cells after indicated treatments. C-D) Survival fraction of U87MG-Luc2 and $\mathrm{E} 2$ cells upon indicated treatments following radiation. Error bars indicate SEM. 
A)

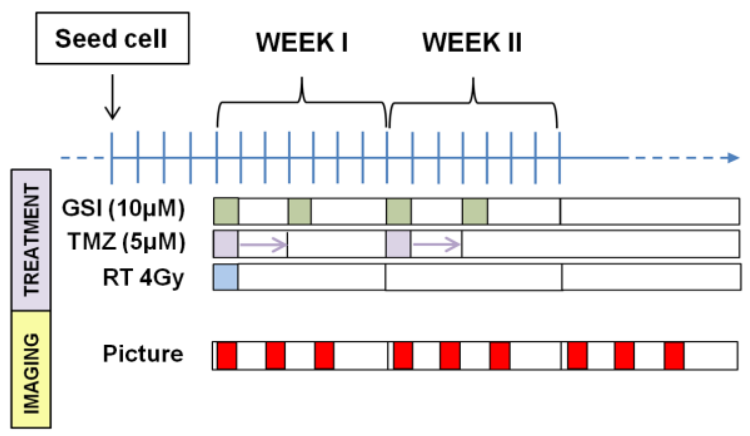

B)

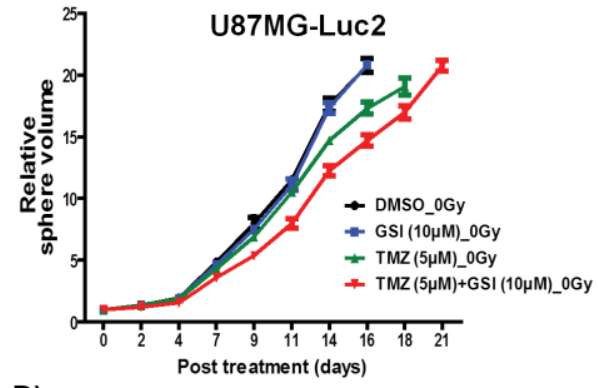

D)

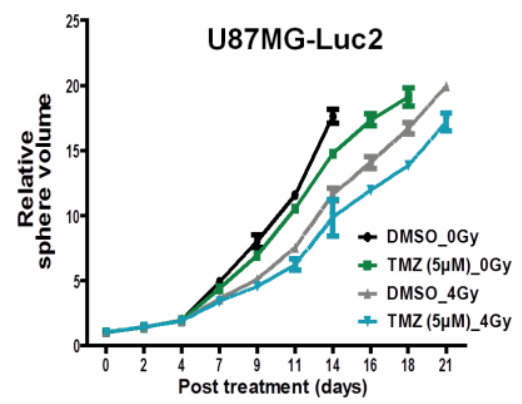

C)

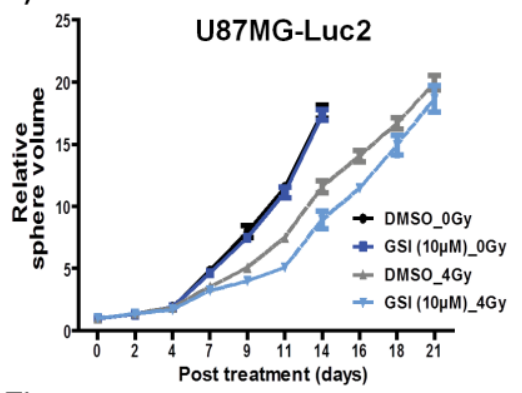

E)

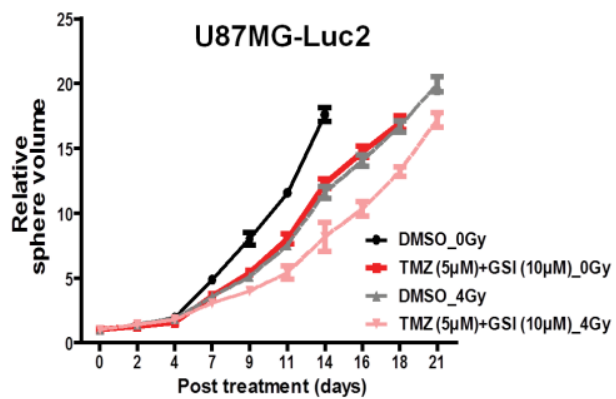

Figure 3: Effect of NOTCH inhibition combined with TMZ and RT on 3D spheroid growth in vitro. A) Schematic of the 3D spheroid assay treatment schedules. B-E) Individual spheroid growth was imaged $3 x /$ week and the volume was calculated after indicated treatments till time to reach 20x starting volume. Spheroid growth demonstrates delays upon combination treatments compared with DMSO as vehicle control. Error bars indicates SEM. 

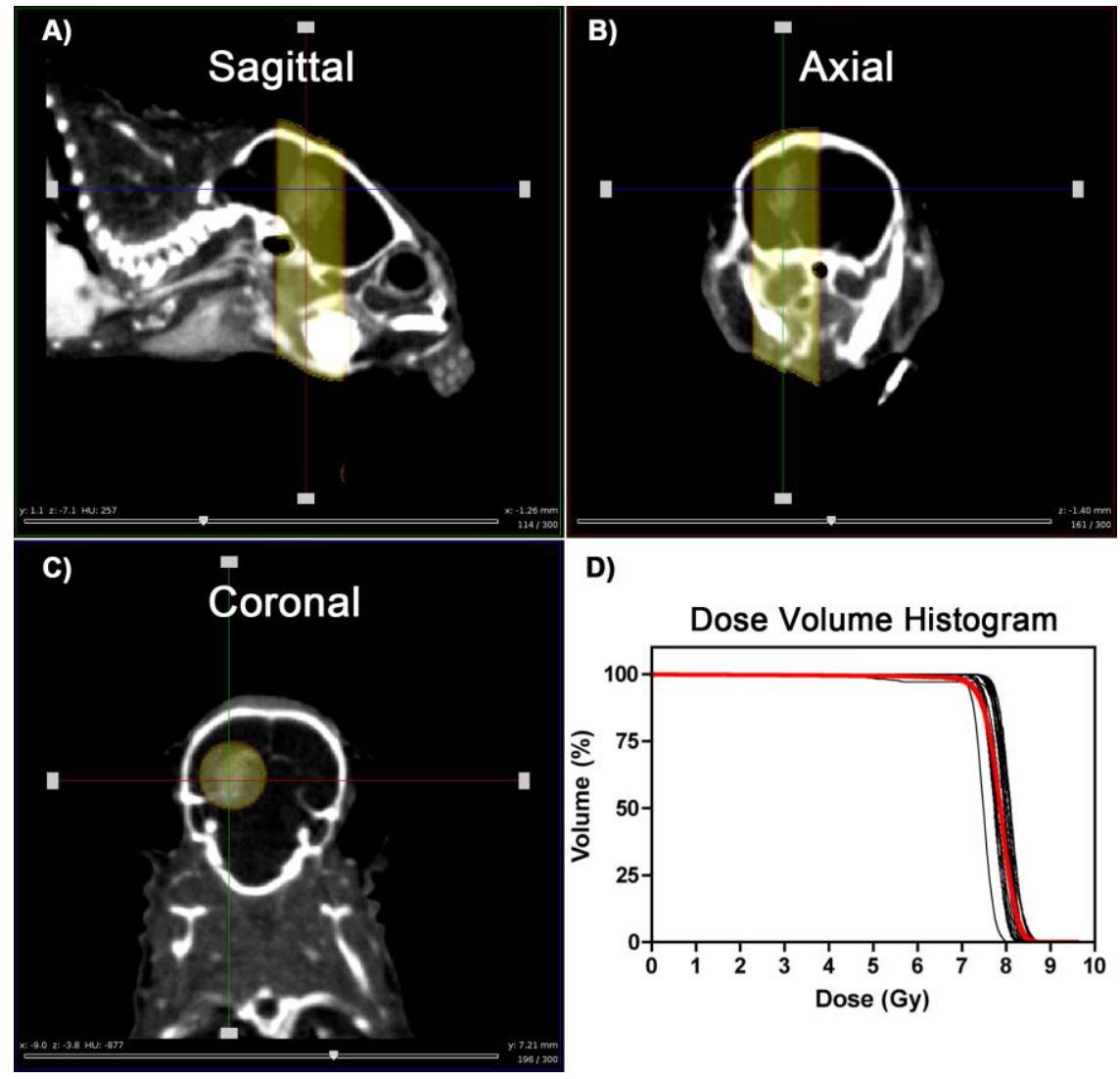

Figure 4: Radiation treatment set-up and the resulting Dose Volume Histograms. A-C) Visualization of the tumor in the brain from different planes (Sagittal, Axial and coronal) and applied parallel-opposed radiation beams to target the tumor. D) Resulting DVHs of the tumors after 8Gy irradiation. Red line shows the average of the DVHs. 
A)
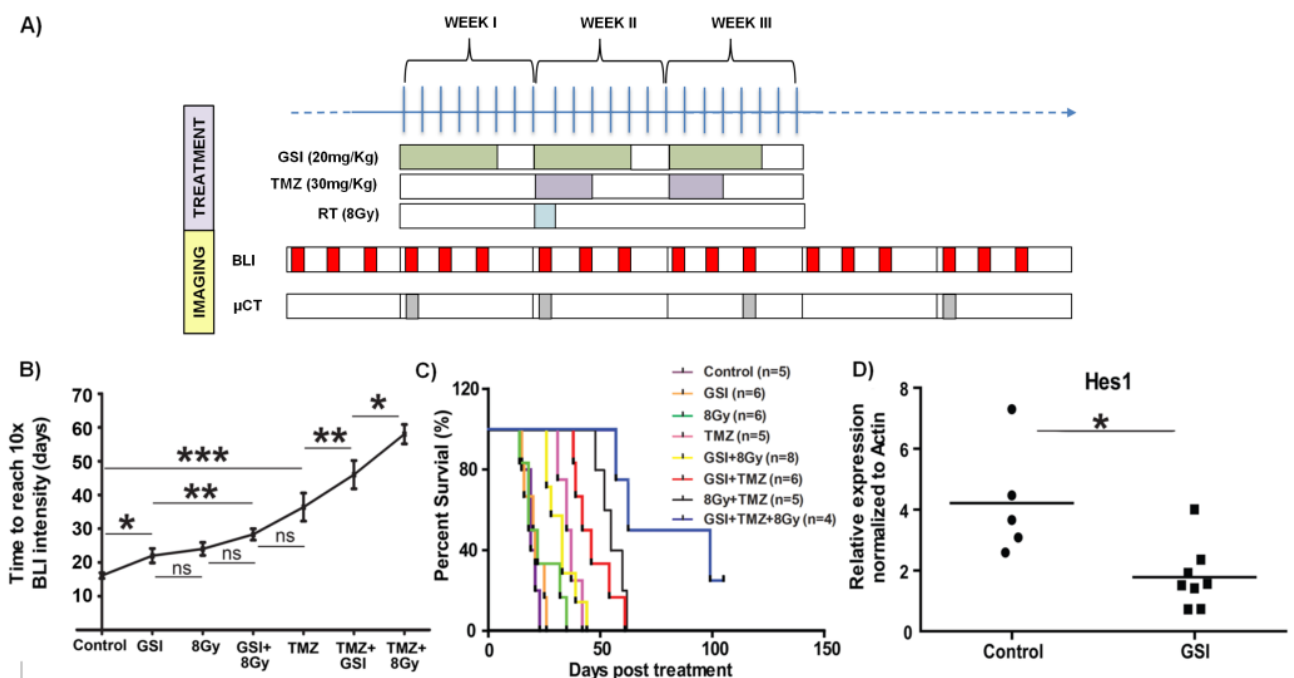

Figure 5: Effect of NOTCH inhibition combined with TMZ and RT in orthotopic U87MG-Luc2 glioblastoma in vivo. A) Schematic of the treatment schedules in vivo. B) Tumor growth delay upon indicated treatments measured by BLI signal intensity for mice in each treatment group and the end point was when the BLI signal intensity reached to $10 x$ intensity of the starting treatment day. C) Kaplan-Meier survival curve indicates number of days mice survived post-treatment. End-point was assessed based on the neurological sign and weight loss. D) mRNA expression of the HES1 expression in tumor samples treated with GSI $v s$. control was determined by qRT-PCR. Values were normalized to actin. Error bars indicates SEM. $\left(* \mathrm{p}<0.05,{ }^{* *} \mathrm{p}<0.01\right.$ and $* * * \mathrm{p}<0.001$, ns: not significant). 
A)
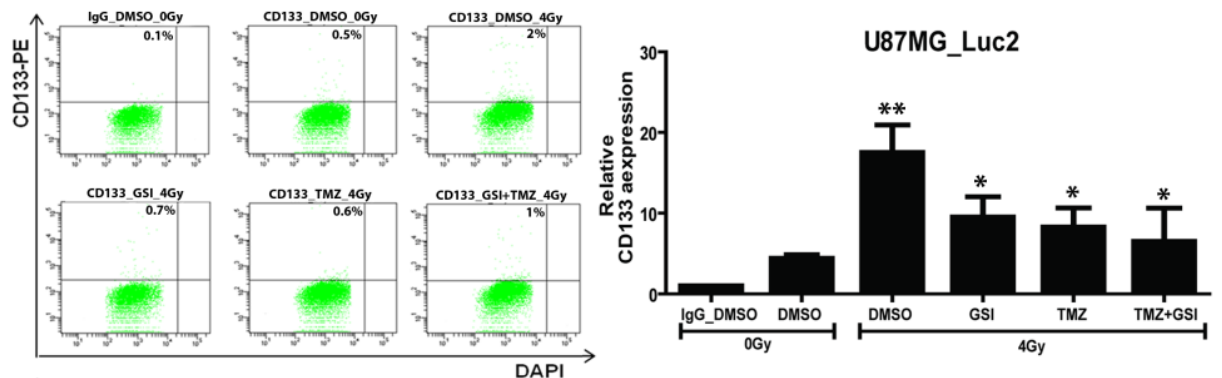

B)
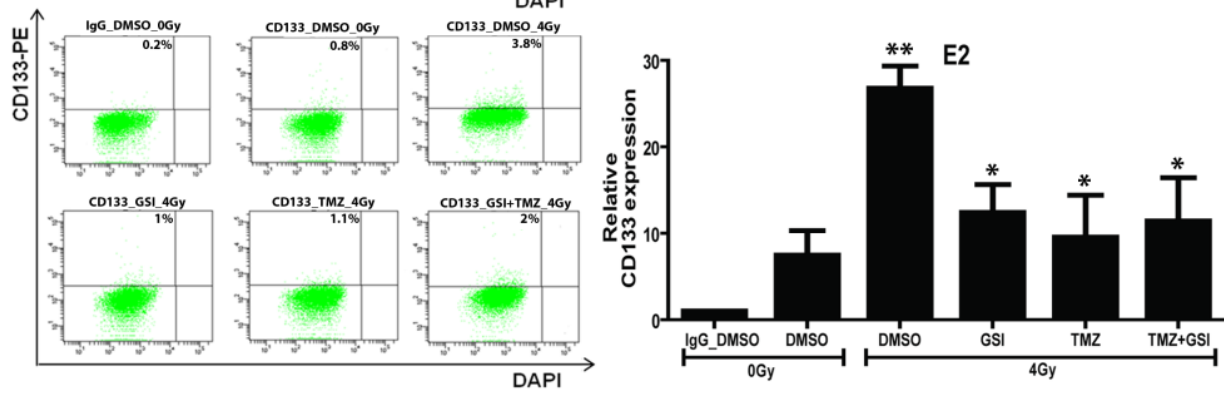

Figure 6: Effect of NOTCH inhibition combined with TMZ and RT on CD133 expression. A-B) The expression of CD133 marker upon indicated treatments in U87MG-Luc2 and E2 cells was measured by flow cytometry. Graphs indicate the quantification of the relative CD133 expression normalized to $\mathrm{IgG}$ control upon indicated treatments. DAPI was used for the live cell staining. Concentration used in all cases was $10 \mu \mathrm{M}$ GSI and $10 \mu \mathrm{M}$ TMZ. Error bars indicate SEM. $(* \mathrm{p}<0.05$, ** $\mathrm{p}<0.01$ and ***p $<0.001)$. 


\section{Supplementary information}

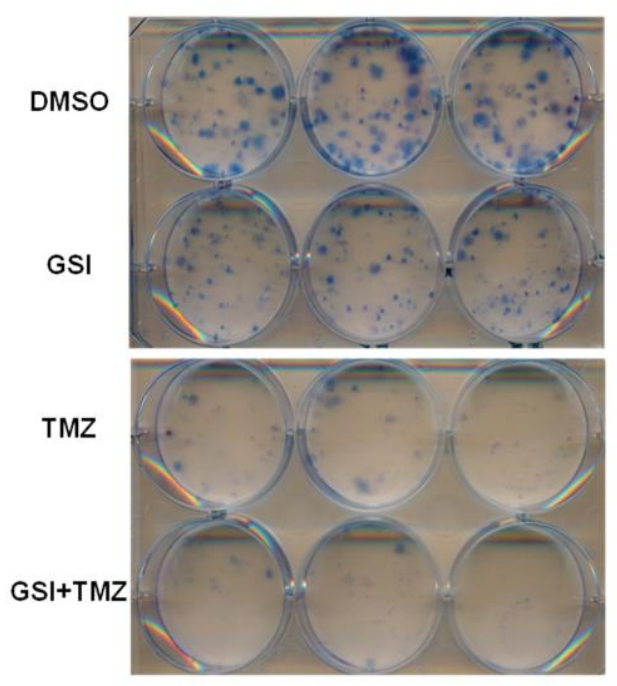

Supplementary Figure 2: E2 clonogenic assay. Representative image of the effect of GSI and TMZ treatment following radiation in E2 cells as measured by clonogenic assays.

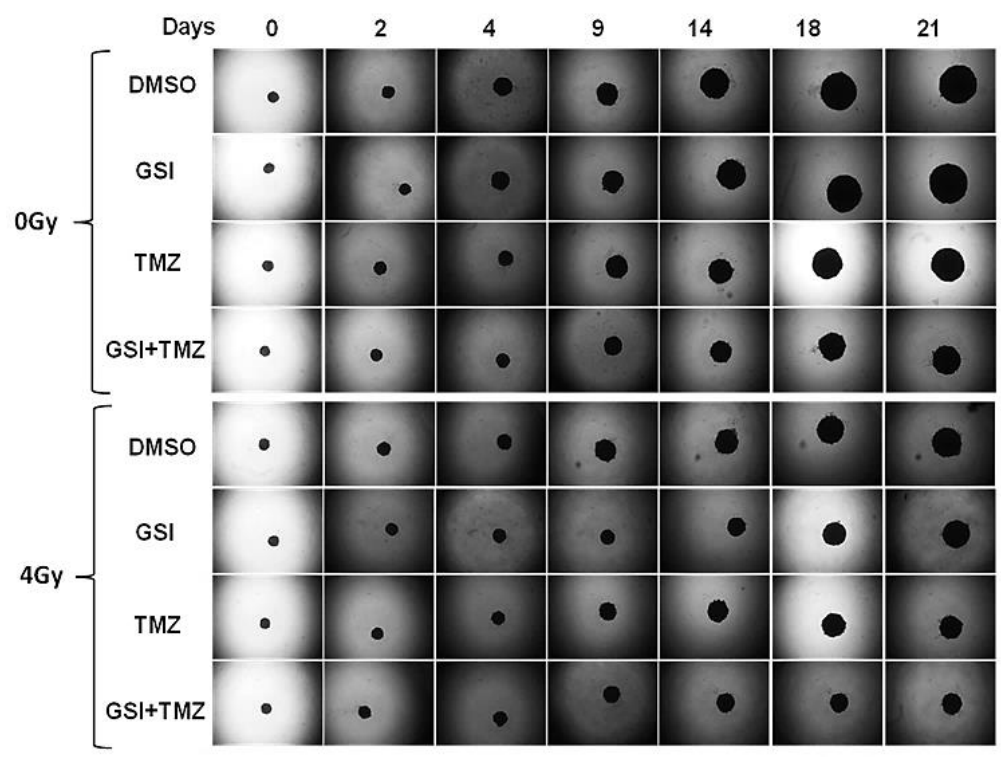

Supplementary Figure 2: U87MG-Luc2 spheroid assay. Representative image of the 3D spheroid upon indicated treatments over time. 

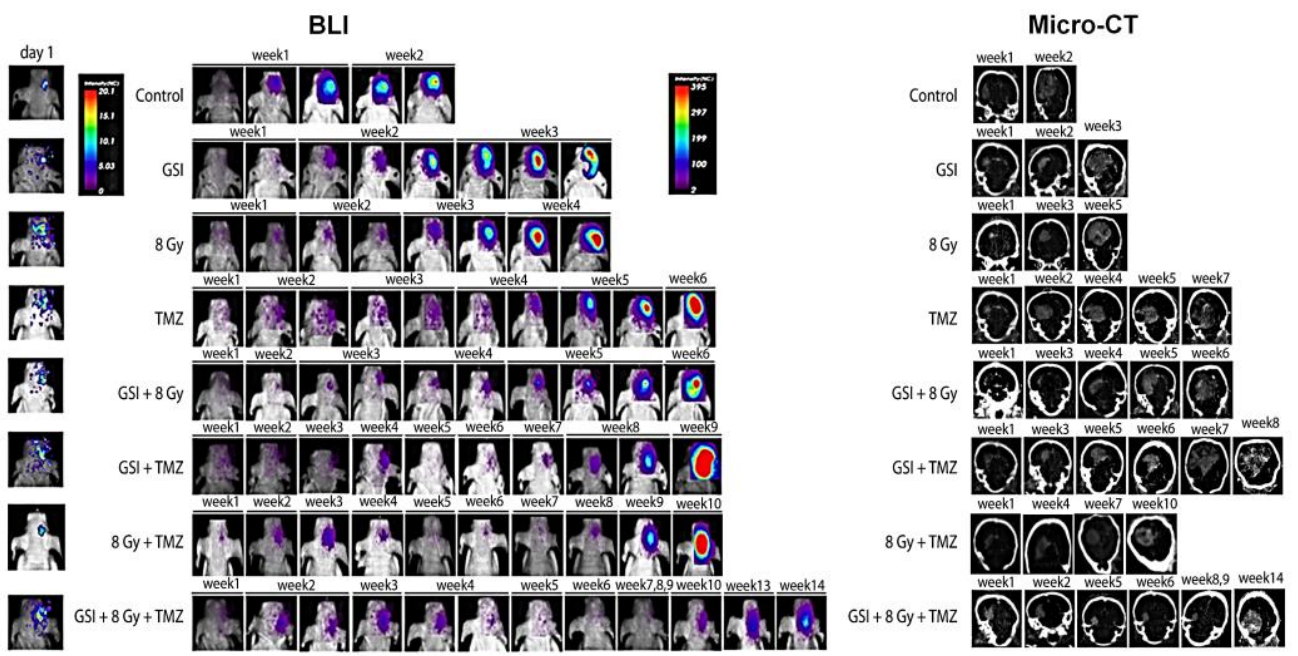

Supplementary Figure 3: Following tumor growth using BLI and/or micro-CT. Representative BLI and contrast enhanced micro-CT images of different treatment groups at different time points post-treatment.

A)

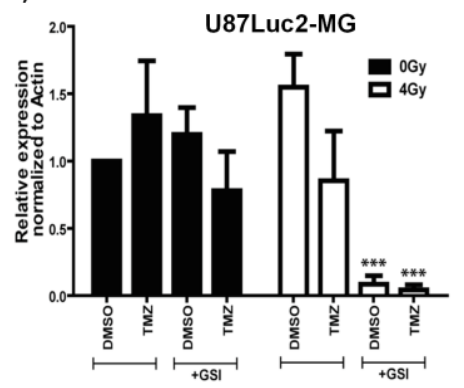

B)

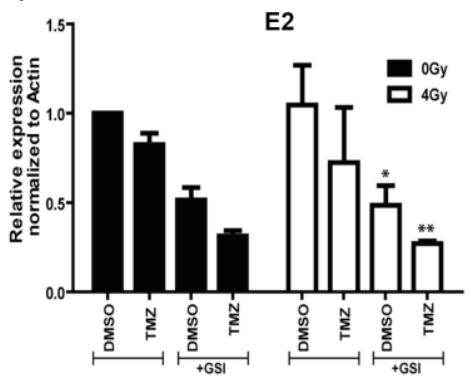

C)

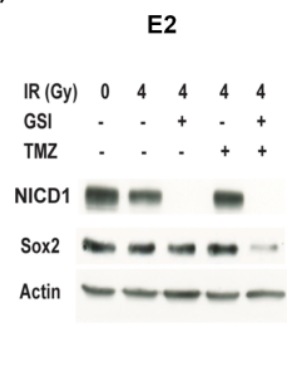

Supplementary Figure 4: Effect of NOTCH inhibition combined with TMZ and RT on SOX2 expression. A-B) mRNA expression of the SOX2 stem cell marker in U87MG-Luc2 and E2 cells upon indicated treatments was measured by qRT-PCR Error bars indicate SEM. C) Protein expression level of SOX2 and active form of NOTCH1 (NICD1) in E2 cells upon indicated treatments was analyzed by Western blotting. Actin serves as loading control. Concentration used in all cases was $10 \mu \mathrm{M}$ GSI and 10 $\mu \mathrm{M}$ TMZ. Error bars indicate SEM. $(* \mathrm{p}<0.05, * * \mathrm{p}<0.01$ and $* * * \mathrm{p}<0.001)$. 



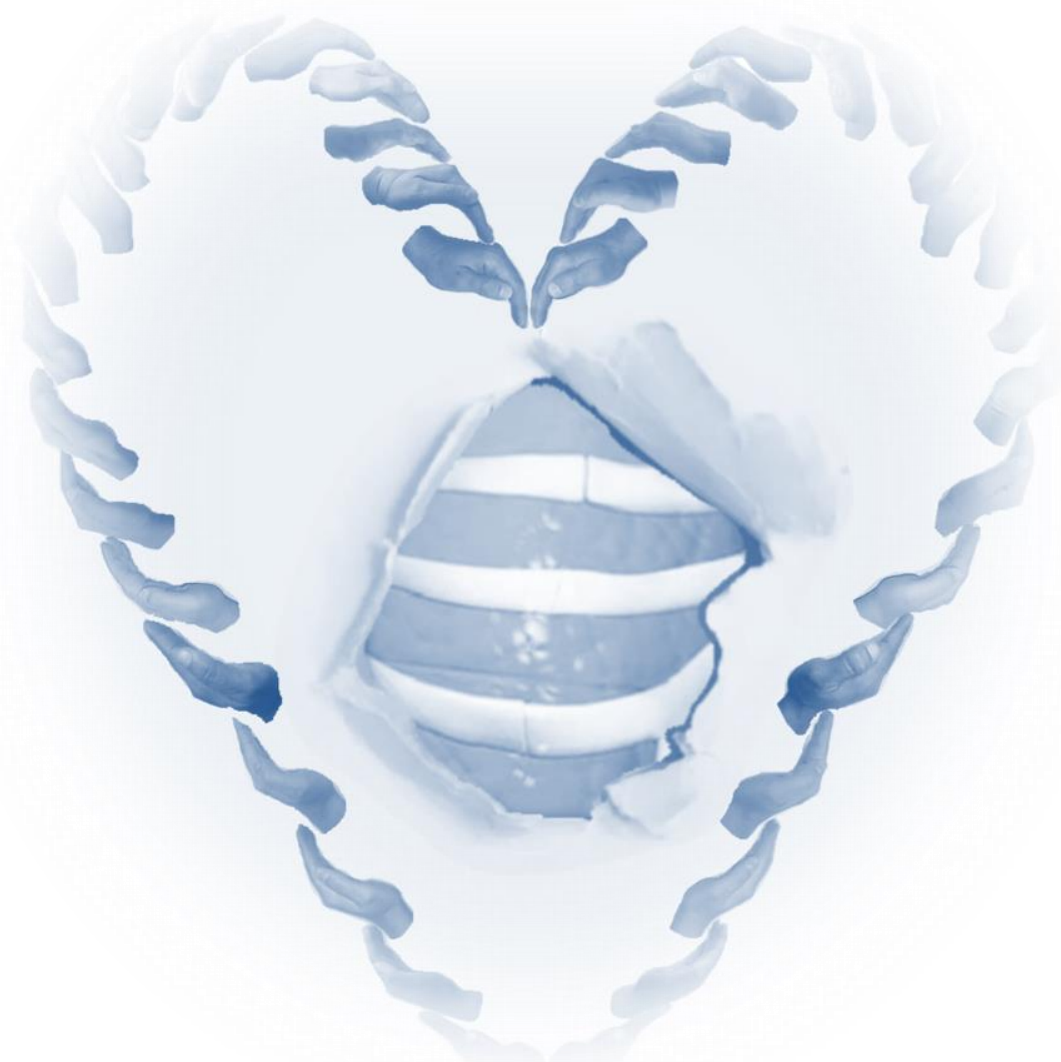




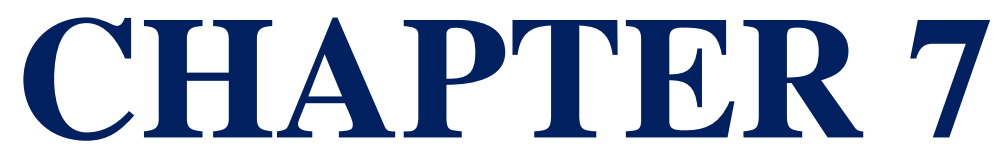

General discussion and Future Perspectives 


\section{TABLE OF CONTENTS}

THE SEARCH FOR "ORDO AB CHAO"

TREATMENT RESISTANCE AND NOTCH SIGNALING 239

THE ERA OF PERSONALIZED MEDICINE 240

IMPROVEMENT OF PRECLINICAL MODELS 242

- 2D MONOLAYER ASSAYS WITH TUMOR CELL LINES 242

- 3D SPHEROID MULTICELLULAR ASSAYS 243

- IN VIVO MODELS IN RODENTS 244

- ECTOPIC SC XENOGRAFT IMMUNE COMPROMISED MODELS 244

- ORTHOTOPIC XENOGRAFT IMMUNE COMPROMISED MODELS 245

- Metastatic MODELS 246

- HUMANIZED MOUSE MODELS 246

IS A NSCLC CURE FEASIBLE WITH NOTCH INH. THERAPY? 246

REFERENCES $\quad 248$ 


\section{The search for "ordo ab chao"}

The "Edwin Smith Papyrus" dated back to 3000 B.C. in ancient Egypt, has the first written record of what we now call cancer, a term fathered by Hippocrates (460-370 B.C.) from the Greek work "karkinos" which means crab (due to the finger-like projections characteristic of cancerous growth). Several cases of breast "ulcers" were documented, removed by cauterization, and it was remarked: "There is no treatment". Where are we now? Five millenniums later, cancer continues to coexist, and its eradication is not on the horizon. What about its elimination?

The first documentation of lung cancer dates back to 1761. Since then, several key discoveries have aided in the understanding and treatment of lung cancer [1]):

1. The use of autopsies to relate disease with pathology (Morgani, Padua 1761),

2. The introduction of surgery as therapeutic strategy for local cancer (Hunter, Scotland 1728-1793),

3. Histological analysis of tissues of patients under the microscope (Virchow, Germany $19^{\text {th }}$ century),

4. Discovery of X-rays (Roentgen 1896; Nobel Prize 1901) later used for diagnosis and radiation treatment,

5. Linking tobacco use as a cause for lung cancer (1950s, and Verner, London 1620),

6. Establishing of the TNM (primary Tumor, lymph Nodes, and Metastasis) staging system worldwide for cancer (1958),

7. The use of imaging (ultrasound, computed tomography (CT), magnetic resonance imaging (MRI), and positron emission tomography (PET)) to diagnose cancer (1970s),

8. The approval of cisplatin for NSCLC treatment (1978)

9. Sequencing of the first human genome (Lander and Venter 2001),

10. The approval tyrosine kinase inhibitors (gefitinib) in NSCLC (2003),

11. The approval of anti-angiogenesis inhibitor (bevacizumab) for NSCLC (2006),

12. The approval of immune therapy (nivolumab, pembrolizumab) for first-line treatment of advanced lung cancer (2015).

These discoveries have set the basis for further research in the field of surgery, chemotherapeutics, cancer genetics and biological and targeted therapies, immunotherapy, radiotherapy, and combinatory treatments thereof.

The Hippocratic view of cancer as an incurable disease has been modified since its conception. Almost five decades ago, $\sim 50 \%$ of patients diagnosed with cancer survived after treatment for at least 5-years, nowadays, more than two-thirds survive 
that long. Fortunately, part of the medical attention has been redirected to quality of life management of cancer survivors prolonging outcome. However, despite these improvements, cancer took 8.8 million deaths in 2015 worldwide, out of which 1.69 million were due to lung cancer, the predominant cancer-related death worldwide (World Health Organization) and mortality is estimated to increase. There are several risk factors known for developing cancer: 1) Genetic (inheritable or sporadic mutations); 2) Behavioral/dietary (obesity, alcohol consumption and low fiber intake) and 3) Environmental/physical (UV, ionizing radiation), chemical carcinogens (tobacco, aflatoxin in polluted food, processed meat and red meat, arsenic in drinking water) and biological agents (e.g. bacterial, viral, or parasitic infections). Despite the scientific knowledge in cancer causes and biology, the increase in cancer survival is not proportional to the amount of treatment options available. This is due to two major obstacles: late diagnosis and the appearance of treatment resistance.

Chest pain, dyspnea (shortness of breath), cough fatigue, sweating, pneumonia, hemoptysis, fever, chills, and body weight loss are all symptoms of lung cancer, some of which are shared with other milder diseases such as: common cold, food poisoning, asthma, bronchitis, and anaphylaxis, therefore misdiagnoses occur frequently. Over two-thirds of lung cancer patients are diagnosed at advanced stages (III-IV) where curative surgery is challenging due to spread throughout the lungs and lymph nodes or even metastases in other organs (e.g. brain, bones, liver, kidney or adrenal glands). Low-dose CT screening of populations at high risk of lung cancer occurrence could potentially increase the chance of early detection, however, it is not optimized yet for large-scale clinical implementation. Parameters such as precise definition of the high-risk population, timing and frequency, type of CT (2D versus 3D), management of false positive cases, and cost-effectiveness, as compared to smoking cessation, require attention before proper assessment of its usefulness for clinical practice [2].

Therapies with curative intent at advanced stages mostly rely on chemotherapy or chemoradiation if performance status is not compromised, and on targeted therapies or immunotherapies when cancers are addicted to certain oncogene driver mutations. Regardless of treatment-type, the majority of cancers develop resistance to treatment causing relapse and tumor progression and increasing mortality. In this chaos, is it possible to find order ("ordo ab chao")? Often the answer to problems lies in their simplification into smaller, manageable parts so, instead of looking at the big picture of "LUNG CANCER", let's break it down a NOTCH. 


\section{Treatment resistance and NOTCH signaling}

Standard first line treatment for inoperable locally advanced stage III NSCLC is concurrent polychemotherapy with fractionated radiation, 60Gy in 2Gy fractions [3], where chemotherapy usually involves a doublet where one is platinum-based [4]. Cisplatin and carboplatin have mechanistic similarities and a broad spectrum of action in a diversity of cancers. It has been suggested that cisplatin provides a survival advantage over carboplatin [5] but with a more pronounced toxicity profile including nephro-, neuro- and ototoxicity. Carboplatin on the other hand, is associated with significant myelotoxicity [6]. NSCLC patients treated with platinumbased drugs often relapse because treatment becomes ineffective, and further lines of therapy have to be considered. Several resistance-mechanisms have been identified such as genetic mutations that confer a proliferative advantage, drug efflux systems such as $\mathrm{ABC}$ drug transporters, glutathione-dependent enzymes countering ROS, topoisomerases, $\mathrm{O}^{6}$-methylguanine-DNA methyltransferases and growth factors [7]. Some of the considerations taken for second-line therapy include not only the type of therapy, response, and toxicity of the first-line treatment, but also the performance status, the presence of comorbidities, gender, race, age, smoking status, and patient's choice before entering further lines of therapies.

Given the high extent of inter- and intra-patient heterogeneity in lung cancer it is evident that this underlies the temporal tumor regressions but ultimately relapse and progress. We now understand that this heterogeneity is driving the survival of subpopulations of tumor cells with stem cell properties -cancer stem cells- that underlie intrinsic and acquired resistance, and that current treatment fails to eradicate these tumor cells. In this thesis, we have concentrated our efforts in understanding how the NOTCH signaling pathway is involved in treatment resistance and how targeting this pathway might be a complementary therapy option. In chapter $\mathbf{2}$, we have reviewed the evidence pertaining to a role for NOTCH signaling in primary or acquired resistance to chemotherapeutics or targeted agents used in NSCLC. NOTCH signaling crosstalks with multiple pathways and hence, enhances tumor resistance in different ways such as maintaining cancer stem cells, upregulating $\mathrm{ABC}$ drug transporters, inducing epithelial-mesenchymal transition or endothelialmesenchymal transition, promoting hypoxia tolerance, downregulating TP53, upregulating PI3K/Akt, RAS or the growth factors IGFR, EGFR and VEGF. Upregulated NOTCH signaling occurs in $\sim 40 \%$ of lung adenocarcinomas, $10 \%$ due to NOTCH1 gain of function mutations and $30 \%$ due to loss of the negative regulator of the NOTCH receptor, NUMB [8]. There is ample preclinical evidence for long lasting anti-tumor activity in lung cancer with small molecule $\mathrm{NOTCH} / \gamma$-secretase 
inhibitors (GSI) [9] or monoclonal antibodies against NOTCH receptors or DLL4 ligands in combination with diverse chemotherapy, radiotherapy and targeted drugs. Our work has demonstrated that NOTCH signaling also promotes resistance to radiation therapy [10] and that GSI are effective in combination treatments with chemotherapy and radiotherapy [11] (chapter 6).

Active NOTCH signaling has been linked in different cancers to poor prognosis but also to resistance towards a broad range of chemotherapeutics including cisplatin [12], docetaxel [13], gefitinib [14], anti-HER2 (human epidermal growth factor receptor 2) [15], anti-estrogens [16], dasatinib [17], temozolomide [11], doxorubicin and melphalan [18]. Therefore, targeting NOTCH could be a promising strategy not only for lung cancer but also for other types of cancer. In chapter 3, we developed 2D and 3D cell models to identify novel treatment combinations of chemotherapy, radiation therapy and a NOTCH inhibitor for non-squamous non-small cell lung cancer. We observed that for most of the tested compounds, addition of the panNOTCH GSI (BMS-906024) to clinically relevant concentrations of chemotherapy or chemoradiation regimens resulted in a synergistically enhanced 3D tumor spheroid specific growth delay. To our knowledge, there have been no further studies with these triplets. Our studies however, assess long-term survival after multiple populations doublings using concentrations of chemotherapeutics that are achievable in patients.

\section{The era of personalized medicine}

Personalized medicine is not a pioneer concept, Hippocrates already stated in his time: "It is far more important to know what person the disease has than what disease the person has". However, it wasn't until the sequencing of the hu man genome in $2001[19,20]$ that the possibility of implementing it became a reality. The birth of personalized medicine began in 2005 (and has thrived since then) with the generation of the "Omics" data [21] such as genomics [22], transcriptomics [23], proteomics [24, 25], metabolomics [26], epigenomics [27, 28] and radiomics [29]. Also, molecular and tumor hypoxia imaging using PET/CT, HX4, and MRI has contributed to improved diagnosis, patient selection and treatment monitoring [3032]. Nowadays, tumor biopsies can be sequenced in order to detect genes that could be causing oncogene addiction in cancer and/or could be implicated in resistance mechanisms such as mini real-time PCR tests for KRAS genotyping, dichotomous immunohistochemical stain and Fluorescence in situ hybridization for ALK rearrangement detection, non-invasive imaging such as tumor hypoxia PET imaging to apply radiation dose painting strategies [33], and the analysis of cell-free 
circulating tumoral DNA in liquid biopsies for EGFR /KRAS mutations. However, clinical application of these diagnostic tests remains challenging and should be referred to in the ESMO (European Society of Medical Oncology) guidelines [34]. To date, there are no tests clinically implemented for NOTCH signaling however, in chapter 2 we comment on the possibility of screening NOTCH pathway target genes in the blood and the hair follicles and in chapter 4 we suggest a potential biomarker found in serum for NOTCH activity (e.g. thymic stromal lymphopoietin, TSLP), although further validation is required.

In combination with improved sequencing techniques, WHO has performed a meticulous histology-based classification of lung cancer into over 70 different subtypes [35]. How does this improve our current understanding resistance to therapy? In chapter 2 we have discussed that genome profiles between lung adenocarcinomas and squamous cell carcinomas vary not only based on the mutation types but also in the frequency of the known mutations. This explains why different cancer subtypes are not equally responsive to the same therapy. For example, (chapter 2), comparing NSCLC patients with EGFR mutations, lung adenocarcinoma patients are more likely to benefit from EGFR-TKI therapy than squamous cell carcinoma in terms of objective response rates, overall survival, and progression-free survival [36]; $[37,38]$. Moreover, the brochioloalveolar subtype is the most likely to benefit from gefitinib treatment than the adenocarcinoma subtype [39]. Squamous cell carcinoma patients, in contrast to adenocarcinoma patients, have similar responses to both types treatment combinations, and could benefit more from a cisplatin plus etoposide treatment than a four-drug combination (cyclophosphamide, adriamycin, methotrexate, procarbazine)[40]. Large cell neuroendocrine carcinomas and small cell lung carcinomas have similar biological behaviors and respond similarly to some treatments: irinotecan, platinum, and taxanes, which are more effective than pemetrexed [41-43].

With genome sequencing, the treatment landscape of lung cancer patients is changing from systemic therapies using chemotherapy and radiotherapy to subtype specific mutation analysis for targeted therapeutics, although this strategy is similarly challenging due to the appearance of therapeutic resistance. For example, the tyrosine kinase inhibitor gefitinib, was initially FDA-approved in 2003 for the treatment of non-small cell carcinomas but was stopped again in 2005 due to lack of clinical activity in the setting for which it was approved [44]. Gefitinib approval was regained in 2009 when clinical activity was demonstrated after stratification of patients according to EGFR mutation status [45]. However, cancers became resistant to EGFR tyrosine kinase inhibitors by acquiring the T790M mutation (in the gefitinib binding pocket of EGFR) fueling $2^{\text {nd }}$ and $3^{\text {rd }}$ generation inhibitors with improved 
response rates leading to approval of Osimertinib [46]. Recently, resistance against Osimertinib has been observed as well (C797S mutation) [47] emphasizing that tumor evolution is a continuous process and that treatments imposes a selection pressure for the development of resistant subclones. Recent studies have postulated (as discussed in chapter 2) that "cancer mutation signatures" are more predictive for treatment response than single mutation status [48] and other groups have reported that they are predictive of prognosis [49]. This brings us back the essence of cancer development: the multistep acquisition of mutations in gene pathways, encompassing the hallmarks of cancers, driving the adenoma-carcinoma sequence leading to invasive metastatic cancer [50]. Cancer genome sequencing has confirmed that human tumors are under continuous selection pressure and that multiple independent 'clones' arise and survive following linear and branched evolution models [51].

\section{Improvement of preclinical models}

In order to test the combinations of therapies that improve the survival rate of patients, it is essential to have physiologically relevant, controlled, unbiased models in which to test such therapies. Such models undeniably are humans. Because it is unethical to test drugs indiscriminately on humans of which we know nothing of, we should reach a compromise in less optimal models prior for human studies. There are several models that can aid in the matter, some of which are the following:

\section{D monolayer assays with tumor cell lines}

Cytotoxicity assays in cells growing in monolayer are fast and can be used for highthroughput screening of compounds and have permitted great insight into the tumor metabolism and biology. However, this model fails to mimic the complexity and heterogeneity of clinical tumors, the organization and architecture, areas of limitedoxygen diffusion, cell-cell interactions, and the interaction with the tumor microenvironment and the immune system.

Using 2D models, we showed in chapter 3 that different non-squamous NSCLC cell lines have different responses to chemotherapeutics with/without radiation treatment. Screens in $\mathrm{H} 1299$ and $\mathrm{H} 460$ suggest that 21\% versus 5\% were synergistic, and $17 \%$ versus $11 \%$ were additive chemoradiation interactions, respectively. Etoposide, pemetrexed, and docetaxel were enhanced synergistically, whereas the small molecule ALK inhibitor crizotinib was enhanced additively by RT in the H1299. In 
the H460, results suggest that crizotinib and etoposide interacted synergistically, whereas pemetrexed and docetaxel interacted non-synergistically with RT. Additionally, the effect of carboplatin, and paclitaxel, was only effective in H1299. Interestingly, some of the therapeutics found to be enhanced in both cell lines in response to chemoradiation are currently not part of standard of care for lung cancer in Europe such as irinotecan, mitotane and dasatinib. Irinotecan, however, has shown in Japanese lung cancer patients an increase in survival rates in combination with cisplatin compared to standard etoposide/cisplatin treatments [52]. Further studies are needed to determine potential benefits/toxicities of these combination treatments. Additionally, in chapter 3 and chapter $\mathbf{6}$ we showed that addition of NOTCH inhibition to chemoradiation (with paclitaxel for lung cancer and temozolomide for glioblastoma) attenuates proliferation and reduces clonogenic survival. Additionally, in chapter 6 we found that the glioma stem cell markers CD133, SOX2 and Nestin were reduced in the triple combination treatments and reduced tumor growth in vivo.

\section{D spheroid multicellular assays}

3D models have been shown to better recapitulate tumor physiology in terms of oxygen, nutrients and metabolite gradients reflecting heterogeneity in cell proliferation and cell death, cell-cell interactions and sensitivity to radiation and chemotherapy [53]. In chapter 3, we showed that H1299 specific spheroid growth delay (SSGD) for cisplatin, etoposide, and crizotinib increased significantly $(\mathrm{p}<0.05)$ after the addition of the NOTCH inhibitor (BMS-906024) and for cisplatin, etoposide, paclitaxel, docetaxel, and crizotinib in combination with radiation and BMS-906024. Synergistic interactions $(\mathrm{p}<0.05)$ were observed between BMS906024 and chemoradiation (cisplatin, paclitaxel, docetaxel, and crizotinib). Similar results were observed for $\mathrm{H} 460$ spheroids using paclitaxel or crizotinib in triple therapy versus chemoradiation, and for crizotinib plus BMS-906024 versus crizotinib. In chapter 6, we additionally showed that in U87 glioblastoma 3D multicellular spheroid growth was delayed when the NOTCH inhibitor RO492909 was added to temozolomide plus radiation. This sensitivity to radiation was related to a reduction in the CD133-expressing glioma stem cells that have been implicated in treatment resistance. 


\section{In vivo models in rodents}

Rodent models permit easy handling (small size), and statistical power (greater numbers). Rodents are inexpensive to maintain, they reproduce fast and have large litters, and can be genetically manipulated. They have been proven useful in cancer gene discovery, therapeutics, in vivo imaging, and biomarker discovery. However, there are limitations as well, including specie-specific differences therefore, rodents are not always able to mimic human disease syndromes and cancer predisposition phenotypes. Mice have differences in drug penetration and metabolism compared to humans, thus changing drug pharmacokinetics and dynamics. The mouse diverges in size, life span, organ morphology and physiology, to humans. Telomerase enzyme is active in mice whereas it is minimally active in human adults, therefore mice cells transform and immortalize faster than human cells. Mice tumors require less genetic alterations than humans which occur mainly in germ line or a big proportion of somatic cells while in humans, the first are rare, and the second are sporadic. Mouse tumors are also less heterogeneous than human tumors, and have differences in drug affinity and metabolism which may affect responses, hampering identification of the most suited drugs for patients [54]. In order to avoid, at least partly, these limitations, human tumors can be grown in vivo using immune-compromised mice. There are still several options of models depending on the aim of the study [55].

\section{Ectopic subcutaneous xenograft immune compromised models}

Tumor cells are injected subcutaneously in the hindleg of mice. This model is standard in oncology drug testing studies since tumor volume and growth can easily be measured over time, radiation can be delivered to the tumor only by using a lead shield to avoid major toxicities to other organs. It is a model easy to work with, reproducible, and since tumor volumes may reach up to $2 \mathrm{~cm}^{3}$, the dynamics of tumor hypoxia can be studied over time. However, because mice are immune compromised, tumor interactions with the immune system are lacking. Moreover, because tumors develop in a non-native environment, tumor host tissue interaction in the hindleg are different that the interactions in the tissue from which the tumor originated. Also, mouse and human growth factor signaling and ECM may differ and therefore human tumors may respond differently in the mouse than in man.

With this model, we showed in chapter 4 that NSCLC H460-NOTCH ${ }^{\text {High }}$ tumor model (H460 overexpressing an oncogenic form of NOTCH1) is more sensitive than the H460 wildtype model to cisplatin treatment and may benefit in terms of specific growth delay (SGD) and survival, as opposed to the H460 wt model, by the 
combination with GSI. We also report, in line with our previous work, that NOTCH expressing tumors are more resistant to radiotherapy than wildtype tumors, despite selecting an RT dose that rendered a considerably strong effect (in terms of SGD) as monotherapy. In line with our previous publication, $\mathrm{H} 460-\mathrm{NOTCH}^{\mathrm{High}}$ tumors grew faster than wildtype tumors. Previously, we reported that $\mathrm{H} 460-\mathrm{NOTCH}^{\text {High }}$ tumors were more hypoxic than wildtype controls explaining why they were more resistant to RT, and further analysis of these tumors revealed that there was additionally an increase in vessel density, although the number of vessels and the vessel perfusion didn't change significantly between models, indicative that the increase in vessel density was probable due to non-functional vasculature, explaining the increase in hypoxia (data not shown). In this study, the addition of GSI in specific scheduling regimens improved GSI monotherapy regimens rendering 2 cures as opposed to no cures in monotherapy in $\mathrm{H} 460-\mathrm{NOTCH}^{\mathrm{High}}$ model. We demonstrate that $\mathrm{NOTCH}$ activity expression levels have an important role in tumor growth and sensitivity to treatment and that blocking it in combination with cisplatin or RT are promising interventions with adequate dosing and scheduling. Follow-up studies combining the three therapies remain to elucidate a potential increase in therapeutic benefit.

\section{Orthotopic xenograft immune compromised models}

In these models, tumors are implanted in the original location from which they were derived, in immune-compromised mice (chapter 5 and 6). This model requires expertise to attain reproducibility and tumor growth monitoring is dependent on noninvasive imaging techniques: computed tomography, magnetic resonance imaging or bioluminescence (using genetically modified tumor cells that express firefly luciferase). These tumors are more alike in progression to humans, and invasive capacity of tumors can be studied [55]. In chapter 5, we established a surgical procedure with $100 \%$ survival post-surgery rates, that enables tumor implantation in the right lung of mice in $60-70 \%$ of the mice. H1299 tumors that grow from this location can be monitored over time using dedicated small animal non-invasive imaging modalities such as bioluminescence imaging (BLI) and cone beam microCT (CBCT). We show that bioluminescent signals of different wavelengths (with different tissue penetrance depths) can be acquired and relative quantification can be used in the future to generate accurate spatial tumor maps. We demonstrated proof of concept using dedicated SmART-Plan software in designing radiation plans for these orthotopic tumors illustrating the effect of different collimators and tumor volume, on the use of tumor margins and of static versus arc beams on dose-volume histograms, tumor coverage and calculated mean doses of RT to the organs at risk. 
In chapter 6, we showed using an image-guided micro-CT and precision radiotherapy platform that a combination of the clinically approved $\mathrm{NOTCH} / \gamma$ secretase inhibitor (GSI) RO4929097 with standard of care (TMZ + RT) reduces tumor growth and prolongs survival compared to dual combinations. We previously demonstrated that BLI can be used to accurately predict tumor volume in GBM and in the future, could better complement CT for longitudinal imaging and perhaps treatment planning, but of course, this requires tumor cells with genetically encoded luciferase reporters which may not always be possible or desired.

\section{Metastatic models}

These models can be obtained by injection of tumor cells via tail vein, orthotopic implantation of tumors with invasive abilities or followed by excision of the primary tumor and follow-up of potential metastasis. In chapter 5 we showed that the A549 and H460 models injected orthotopically as cell suspensions are invasive and could be suited for therapeutic testing of late stages of lung cancer where the tumor has colonized the chest and nearby organs. We did however not focus on these models further.

\section{Humanized mouse models}

The engraftment of human hematopoietic stem cells in $I L 2 r y^{\text {null }}$ mice induces a humanized immune response in mice that is suitable for immunotherapy studies of human tumors [56].

\section{Other models}

There are of course many other models, such as genetically engineered mouse models to model human disease, syngeneic mouse models (e.g. Lewis lung carcinoma) whereby immortalized mouse cancer cells are engrafted into the same inbred immunocompetent mouse strain thus preventing tumor rejection and enabling immunotherapy studies, or spontaneous tumor models where tumors arise in the appropriate tissue environment in a host conditioned by the physiological events of tumorigenesis and progression in immune-proficient mice. Each model has its pros and cons and are best suited to answer different biological questions. 


\section{Is a NSCLC cure feasible with NOTCH inhibition therapy?}

Our results in glioblastoma and in lung cancer models presented in this thesis illustrate that there is potential for combination treatments including $\mathrm{NOTCH} / \gamma$ secretase inhibitors. However, as we discuss in chapter 2 , the current interventional setting to test NOTCH inhibition in clinical trials is sub-optimal. Lung cancer is a complex heterogeneous disease that is likely to benefit from individualized treatment planning. In order to obtain definitive evidence of the therapeutic efficacy of $N \mathrm{OTCH} / \gamma$-secretase therapeutics the following is important:

1. Acknowledging interpatient, intratumor and inter-/intrametastatic heterogeneity

2. Treating patients based on histological subtype and genomic signature

3. Patient stratification based on NOTCH signaling expression

4. Optimal selection of scheduling and dosing to minimize toxicity

5. Considering combining $\mathrm{NOTCH}$ inhibition with glucocorticoid treatment to overcome adverse gastrointestinal toxicity due to on-target NOTCH inhibition (see chapter 2)

6. Using treatment combinations of therapies that interact synergistically

7. Developing prognostic and predictive biomarkers for NOTCH interventions

8. Develop companion biomarkers to enable therapeutic modulation

9. Development and clinical validation of selective NOTCH inhibitors vs GSI

10. Avoiding the use of NOTCH inhibitors that cross the blood-brain barrier for extracranial tumors to prevent potential toxicities

Despite the fact that only a minority of non-squamous NSCLC have expression and or mutation of NOTCH pathway, aberrant NOTCH signaling upregulation correlates with poor outcome. With our understanding of tumor heterogeneity and cancer stem cells, and the demonstrated role of NOTCH in these cells, the impact of NOTCH therapeutics may be broader than what is predicted by whole genome analysis. Single cell gene expression analysis of bulk and tumor stem cells may uncover that NOTCH signaling is active in those subpopulations that are most difficult to eradicate by treatment and responsible for recurrence.

Regardless, who decides who we fight for? Any one person is meritorious of our efforts. Who knows? The next person could be you or me. 


\section{References}

1. Yang, P., Lung Cancer in Never Smokers. Seminars in Respiratory and Critical Care Medicine, 2011. 32(1): p. 10-21.

2. Vansteenkiste, J., et al., Early and locally advanced non-small-cell lung cancer (NSCLC): ESMO Clinical Practice Guidelines for diagnosis, treatment and follow-up. Ann Oncol, 2013. 24 Suppl 6: p. vi89-98.

3. Auperin, A., et al., Meta-analysis of concomitant versus sequential radiochemotherapy in locally advanced non-small-cell lung cancer. J Clin Oncol, 2010. 28(13): p. 2181-90.

4. Delbaldo, C., et al., Benefits of adding a drug to a single-agent or a 2-agent chemotherapy regimen in advanced non-small-cell lung cancer: a metaanalysis. Jama, 2004. 292(4): p. 470-84.

5. Hotta, K., et al., Meta-analysis of randomized clinical trials comparing Cisplatin to Carboplatin in patients with advanced non-small-cell lung cancer. J Clin Oncol, 2004. 22(19): p. 3852-9.

6. Ho, G.Y., N. Woodward, and J.I.G. Coward, Cisplatin versus carboplatin: comparative review of therapeutic management in solid malignancies. Critical Reviews in Oncology/Hematology, 2016. 102(Supplement C): p. 37-46.

7. Lippert, T.H., H.J. Ruoff, and M. Volm, Intrinsic and acquired drug resistance in malignant tumors. The main reason for therapeutic failure. Arzneimittelforschung, 2008. 58(6): p. 261-4.

8. Westhoff, B., et al., Alterations of the Notch pathway in lung cancer. Proc Natl Acad Sci U S A, 2009. 106(52): p. 22293-8.

9. van Tetering, G. and M. Vooijs, Proteolytic cleavage of Notch: "HIT and $R U N "$. Curr Mol Med, 2011. 11(4): p. 255-69.

10. Yahyanejad, S., J. Theys, and M. Vooijs, Targeting Notch to overcome radiation resistance. Oncotarget, 2016. 7(7): p. 7610-28.

11. Yahyanejad, S., et al., NOTCH blockade combined with radiation therapy and temozolomide prolongs survival of orthotopic glioblastoma. Oncotarget, 2016. 7(27): p. 41251-41264.

12. Liu, J., et al., Blocking the NOTCH pathway can inhibit the growth of CD133-positive A549 cells and sensitize to chemotherapy. Biochem Biophys Res Commun, 2014. 444(4): p. 670-5.

13. Huang, J., et al., Notch-1 Confers Chemoresistance in Lung Adenocarcinoma to Taxanes through AP-1/microRNA-451 Mediated Regulation of MDR-1. Mol Ther Nucleic Acids, 2016. 5: p. e375.

14. Liu, J., et al., Lung cancer tumorigenicity and drug resistance are maintained through ALDH(hi)CD44(hi) tumor initiating cells. Oncotarget, 2013. 4(10): p. 1698-1711. 
15. Castagnoli, L., et al., Pathobiological implications of the d16HER2 splice variant for stemness and aggressiveness of HER2-positive breast cancer. Oncogene, 2017. 36(12): p. 1721-1732.

16. Simoes, B.M., et al., Anti-estrogen Resistance in Human Breast Tumors Is Driven by JAG1-NOTCH4-Dependent Cancer Stem Cell Activity. Cell Rep, 2015. 12(12): p. 1968-77.

17. Jeong, Y.J., et al., Breast cancer cells evade paclitaxel-induced cell death by developing resistance to dasatinib. Oncol Lett, 2016. 12(3): p. 2153-2158.

18. Nefedova, Y., et al., Inhibition of Notch signaling induces apoptosis of myeloma cells and enhances sensitivity to chemotherapy. Blood, 2008. 111(4): p. 2220-9.

19. Lander, E.S., et al., Initial sequencing and analysis of the human genome. Nature, 2001. 409(6822): p. 860-921.

20. Venter, J.C., et al., The sequence of the human genome. Science, 2001. 291(5507): p. 1304-51.

21. Ghosh, D. and L.M. Poisson, "Omics" data and levels of evidence for biomarker discovery. Genomics, 2009. 93(1): p. 13-6.

22. Liu, L., et al., Comprehensive genomic profiling of lung cancer using a validated panel to explore therapeutic targets in East Asian patients. Cancer Sci, 2017.

23. Garbett, K., et al., Immune transcriptome alterations in the temporal cortex of subjects with autism. Neurobiology of disease, 2008. 30(3): p. 303-311.

24. Rual, J.-F., et al., Towards a proteome-scale map of the human proteinprotein interaction network. Nature, 2005. 437(7062): p. 1173-1178.

25. Stelzl, U., et al., A Human Protein-Protein Interaction Network: A Resource for Annotating the Proteome. Cell, 2005. 122(6): p. 957-968.

26. Fan, T., et al., Metabolomic and transcriptomic profiling of hepatocellular carcinomas in Hras12V transgenic mice. Cancer Med, 2017.

27. Steinberg, J., et al., Integrative epigenomics, transcriptomics and proteomics of patient chondrocytes reveal genes and pathways involved in osteoarthritis. Sci Rep, 2017. 7(1): p. 8935.

28. Duruisseaux, M. and M. Esteller, Lung cancer epigenetics: from knowledge to applications. Semin Cancer Biol, 2017.

29. Chen, B., et al., Development and clinical application of radiomics in lung cancer. Radiat Oncol, 2017. 12(1): p. 154.

30. Aerts, H.J., et al., Development and evaluation of a cetuximab-based imaging probe to target EGFR and EGFRvIII. Radiother Oncol, 2007. 83(3): p. 326-32.

31. Yaromina, A., et al., A novel concept for tumour targeting with radiation: Inverse dose-painting or targeting the "Low Drug Uptake Volume". Radiother Oncol, 2017. 124(3): p. 513-520.

32. Dubois, L., et al., Specific inhibition of carbonic anhydrase IX activity enhances the in vivo therapeutic effect of tumor irradiation. Radiother Oncol, 2011. 99(3): p. 424-31. 
33. Aerts, H.J., et al., Identification of residual metabolic-active areas within NSCLC tumours using a pre-radiotherapy FDG-PET-CT scan: a prospective validation. Lung Cancer, 2012. 75(1): p. 73-6.

34. Novello, S., et al., Metastatic non-small-cell lung cancer: ESMO Clinical Practice Guidelines for diagnosis, treatment and follow-up. Ann Oncol, 2016. 27(suppl 5): p. v1-v27.

35. Travis, W.D., et al., The 2015 World Health Organization Classification of Lung Tumors: Impact of Genetic, Clinical and Radiologic Advances Since the 2004 Classification. J Thorac Oncol, 2015. 10(9): p. 1243-1260.

36. Noronha, V., et al., EGFR mutations in Indian lung cancer patients: clinical correlation and outcome to EGFR targeted therapy. PLoS One, 2013. 8(4): p. e61561.

37. Shukuya, T., et al., Efficacy of gefitinib for non-adenocarcinoma non-smallcell lung cancer patients harboring epidermal growth factor receptor mutations: a pooled analysis of published reports. Cancer Sci, 2011. 102(5): p. 1032-7.

38. Joshi, A., et al., EGFR mutation in squamous cell carcinoma of the lung: does it carry the same connotation as in adenocarcinomas? OncoTargets and therapy, 2017. 10: p. 1859-1863.

39. Miller, V.A., et al., Bronchioloalveolar pathologic subtype and smoking history predict sensitivity to gefitinib in advanced non-small-cell lung cancer. J Clin Oncol, 2004. 22(6): p. 1103-9.

40. Veronesi, A., et al., Chemotherapy of advanced non-small-cell lung cancer with cyclophosphamide, adriamycin, methotrexate, and procarbazine versus cisplatin and etoposide. A randomized study. Am J Clin Oncol, 1988. 11(5): p. 566-71.

41. Yamazaki, S., et al., Clinical responses of large cell neuroendocrine carcinoma of the lung to cisplatin-based chemotherapy. Lung Cancer, 2005. 49(2): p. 217-23.

42. Sun, J.-M., et al., Chemotherapy for pulmonary large cell neuroendocrine carcinoma: Similar to that for small cell lung cancer or non-small cell lung cancer? Lung Cancer, 2012. 77(2): p. 365-370.

43. Iyoda, A., et al., Treatment options for patients with large cell neuroendocrine carcinoma of the lung. General Thoracic and Cardiovascular Surgery, 2014. 62(6): p. 351-356.

44. Thatcher, N., et al., Gefitinib plus best supportive care in previously treated patients with refractory advanced non-small-cell lung cancer: results from a randomised, placebo-controlled, multicentre study (Iressa Survival Evaluation in Lung Cancer). Lancet, 2005. 366(9496): p. 1527-37.

45. Mok, T.S., et al., Gefitinib or carboplatin-paclitaxel in pulmonary adenocarcinoma. N Engl J Med, 2009. 361(10): p. 947-57.

46. Saad, N., et al., Epidermal growth factor receptor T790M mutation-positive metastatic non-small-cell lung cancer: focus on osimertinib (AZD9291). OncoTargets and therapy, 2017. 10: p. 1757-1766. 
47. Song, H.N., et al., Acquired C797S Mutation upon Treatment with a T790MSpecific Third-Generation EGFR Inhibitor (HM61713) in Non-Small Cell Lung Cancer. J Thorac Oncol, 2016. 11(4): p. e45-7.

48. Alexandrov, L.B., et al., Signatures of mutational processes in human cancer. Nature, 2013. 500(7463): p. 415-21.

49. Nagy, A., et al., KRAS driven expression signature has prognostic power superior to mutation status in non-small cell lung cancer. Int J Cancer, 2017. 140(4): p. 930-937.

50. Hanahan, D. and R.A. Weinberg, Hallmarks of cancer: the next generation. Cell, 2011. 144(5): p. 646-674.

51. Gerlinger, M., et al., Cancer: evolution within a lifetime. Annu Rev Genet, 2014. 48: p. 215-36.

52. Langer, C.J., The emerging world role of irinotecan in lung cancer. Oncology (Williston Park), 2001. 15(7 Suppl 8): p. 15-21.

53. Kimlin, L.C., G. Casagrande, and V.M. Virador, In vitro three-dimensional (3D) models in cancer research: an update. Mol Carcinog, 2013. 52(3): p. 167-82.

54. Cheon, D.J. and S. Orsulic, Mouse models of cancer. Annu Rev Pathol, 2011. 6: p. 95-119.

55. Jung, J., Human Tumor Xenograft Models for Preclinical Assessment of Anticancer Drug Development. Toxicological Research, 2014. 30(1): p. 1-5.

56. Brehm, M.A., L.D. Shultz, and D.L. Greiner, Humanized Mouse Models to Study Human Diseases. Current opinion in endocrinology, diabetes, and obesity, 2010. 17(2): p. 120-125. 


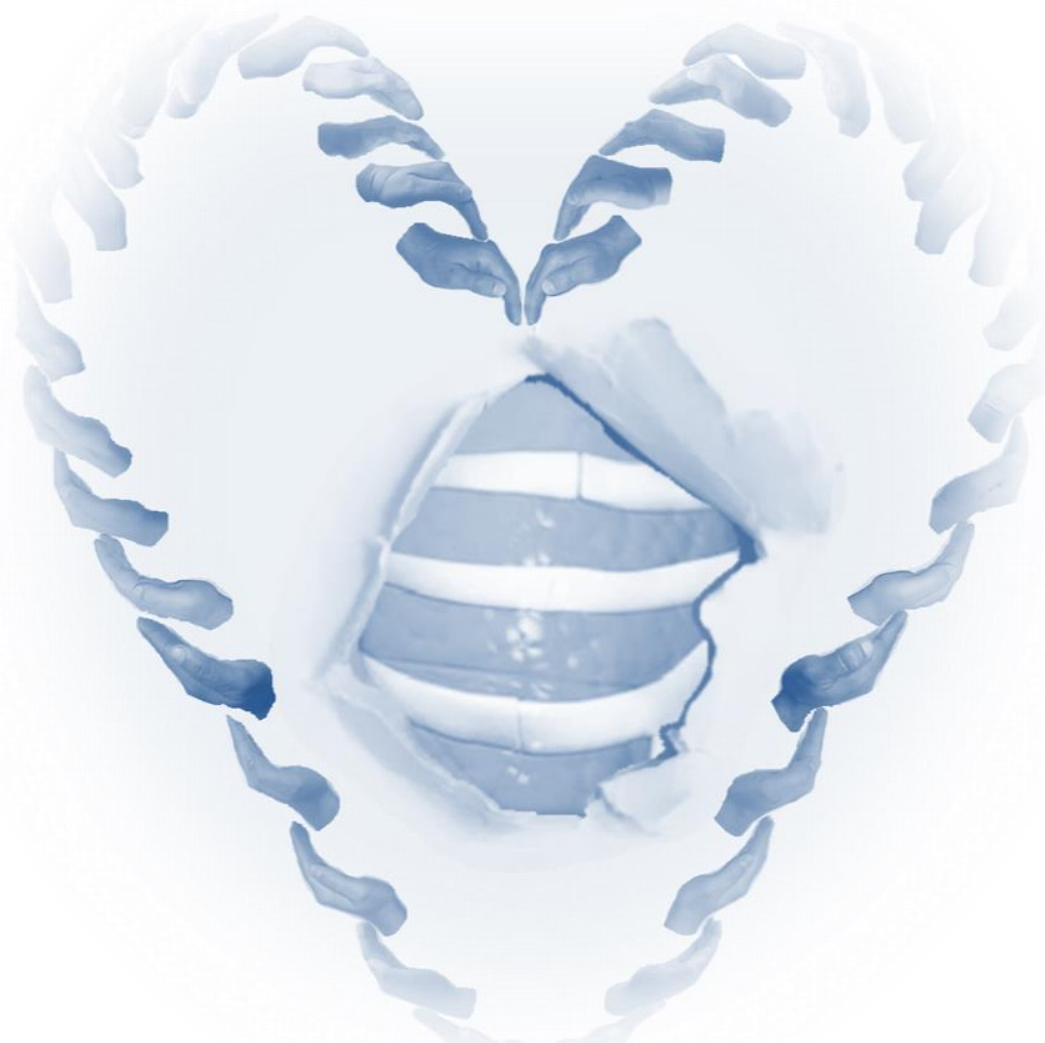


ADDENDUM 
Summary 
In this thesis, we have investigated the potential of NOTCH signaling as a therapeutic target in non-squamous NSCLC and GBM. We developed novel in vitro and in vivo models to investigate whether NOTCH inhibition using small molecule inhibitors (GSI) when used in combination with standard of care conventional treatment with chemotherapy and radiotherapy improves treatment response.

Chapter 2 provides a review on the role of NOTCH signaling in the intrinsic and acquired treatment resistance observed in NSCLC. We discuss how the NOTCH signaling pathway is associated with intrinsic mechanism of tumor resistance including its role in: cancer stem cells, ABC drug transporters, epithelialmesenchymal transition, hypoxia, and crosstalk with other oncogenic pathways such as with TP53, growth factor signaling (e.g. EGFR) tumor angiogenesis (e.g. VEGF) and the DNA damage response (ATM). We conclude that NOTCH-based therapeutic regimens in combination with radiation, and chemotherapy/targeted therapy is a promising strategy to further explore in NSCLC.

In Chapter 3, we developed in vitro in 2D and 3D semi-high throughput drug testing assays to investigate whether NOTCH inhibition enhanced the anti-tumor effect of chemo- and chemoradiation treatments. We screened 101 FDA approved chemotherapeutics from the NCI library combined with RT and focused on those chemotherapies commonly used as part of first line treatment in NSCLC. Synergistic interactions $(\mathrm{p}<0.05)$ were observed between the clinical GSI BMS-906024 and chemoradiation (using cisplatin, paclitaxel, docetaxel, and crizotinib). Several combinations treatments were identified were NOTCH blockade enhanced the cytotoxic and RT effect of these treatments.

In Chapter 4, we describe the development of an in vivo NSCLC tumor xenograft model to test the effect of these single and combined treatments on tumor growth. We identified drug doses of GSI and cisplatin that were effective in tumor growth inhibition but non-toxic. We combined GSI and cisplatin or GSI and RT in tumors with high oncogenic NOTCH activity and those with wildtype NOTCH signaling. Remarkably, we found that $\mathrm{H} 460-\mathrm{NOTCH}^{\text {High }}$ tumors are more sensitive than the H460 wildtype model to cisplatin treatment. In tumors with high NOTCH activity we observed increased vessel density, however these vessels appeared nonfunctional. $\mathrm{H} 460-\mathrm{NOTCH}^{\text {High }}$ tumors are more resistant to radiotherapy than $\mathrm{H} 460$ wildtype tumors and specific scheduling of GSI with respect to RT resulted in tumor cures for both tumor models. 
In Chapter 5, the development of an orthotopic NSCLC preclinical model in mice is described. We tested several models and found that H1299 NSCLC most reproducibly produced tumors as a single localized nodule in the lung. We compared two non-invasive imaging modalities; dual wavelength BLI and dual energy CBCT and found that they correlated. Using these models, we illustrate the effect of different tumor volume-based radiation treatment plan set-ups that can be delivered employing an image-guided, high precision small animal irradiator taking into account tumor margins, radiation dose-volume histogram parameters to both the internal and planned target volume and organs at risk (lung, heart, spinal cord).

In Chapter 6, we investigated the efficacy of NOTCH inhibitors on glioma tumor growth and tumor resistance in vitro and in vivo. Using primary and established glioma cell lines and 3D spheroid models we demonstrate that NOTCH signaling was active but that NOTCH inhibition alone was insufficient to block tumor cell survival. Next, we combined clinically approved NOTCH/ $\gamma$-secretase inhibitor with the standard of care treatment for glioblastoma: concurrent alkylating chemotherapy with temozolomide and radiotherapy. We also tested these combination treatments in an intracranial model from GBM using U87 cells and tested NOTCH inhibitors alone or in combination with standard of care treatment and monitored tumor growth response using non-invasive imaging. We found that similar to our results in vitro, NOTCH inhibition alone did not significantly block tumor growth but that when combined with temozolomide, radiotherapy or both, it significantly prolonged survival. In vitro and in vivo NOTCH inhibition blocked the survival of treatment resistant CD133 cancer stem cells.

Finally, Chapter 7 summarizes the findings of this thesis and comments on the future challenges and opportunities for NOTCH-based interventions in cancer. 
Valorization 


\section{Clinical relevance}

Cancer and cardiovascular diseases are the foremost causes of death worldwide. According to the 2018 report from the American Cancer Society, the 5-year relative survival rate for the combination of all cancers is 70 versus $63 \%$ in white and black races, respectively. Specifically for lung cancer, the 5-year survival rate is $18 \%$ and it can go up to $56 \%$ if it is localized, although only $16 \%$ of lung cancers are diagnosed at an early stage. For glioblastoma multiforme on the other hand, about $5 \%$ survive over 5-years but most patients will survive only for a year. Standard first-line treatment for glioblastoma has been clearly defined since 2005 as a combination of radiotherapy and temozolomide treatment, however, there is still controversy on further lines of treatment. There is a myriad of treatments available to treat cancer, some of which are efficient in initially reducing tumor burden, however, this positive outcome is not always long-lasting and within a few years the cancer recurs. It is this population of resistant cancer stem cells that we have aimed to tackle in this thesis by trying to prolong the effects of current standard of care (chemoradiation) combining it with a resistant cancer stem cell targeting agent: a NOTCH inhibitor. Monotherapy treatments with NOTCH inhibitors have not been successful in clinical trials and many have been halted. However, this effect should have been expected since NOTCH inhibitors only target a small percentage of cells from the bulk of the tumor, and its effects are likely to be potentiated when in combination with therapies that target a characteristic of all cancer cells such as uncontrolled cell division. Evidence of the latter can be seen in this thesis in preclinical studies both in nonsmall cell lung cancer (NSCLC) and glioblastoma (GBM). One of the mayor challenges of NOTCH-based inhibition therapy is gastrointestinal adverse effects, resulting in the induction of diarrhea, due to the requirement of NOTCH protein in normal tissues. Nevertheless, in this thesis we have shown that intermittent scheduling, reduced dosing, and its combination with other therapies with which it synergistically interacts, greatly improves tolerability. We are not pioneers in showing that intermittent dosing reduces the side effects of NOTCH inhibitors. Other groups have also shown that using inhibitors that target a single or two NOTCH receptors different from NOTCH1, also reduces toxicity. In this study, we show that using a NOTCH inhibitor (BMS906024) that targets potently and equally all NOTCH receptors, at a low dose and with an intermittent schedule, is tolerable. The use of pan-NOTCH inhibitors would avoid pre-screening of tumors for the specific $\mathrm{NOTCH}$ receptor that is overexpressed, and a general NOTCH activity screen would be sufficient. In hematological tumors, the use of glucocorticoids in combination the NOTCH inhibition treatment, attenuates the adverse effects upon the gastrointestinal 
tract. Recently, there have been reports of nanoparticles with glucose moieties loaded with $\gamma$-secretase inhibitors, that are internalized by breast cancer cells and stem cells in preclinical studies, where the uptake is correlated with the glycolytic profile of the cells and can efficiently reduce the cancer stem cell population within the tumor (Mamaeva, V et al. Molec Ther 2016; doi: 10.1038/mt.2016.42). These targeteddelivery strategies could help minimize the adverse effects upon normal tissues.

\section{Societal relevance}

Doctors and scientists are continuously on the lookout for new ways to improve treatment, may it be through the accidental discovery of new drugs due to explosions or leaks into the environment; testing of plant, fungi, or animals; comparing the biology of cancer versus healthy cells; or by in silico simulation of the interaction between the potential new drug with its target. These new drugs are then tested first in vitro and later in vivo in two or more animal species, a process that will take $\sim 3-4$ years, to evaluate whether the drugs do what they were designed for, and the potential side effects they may have, thus aiding in translation to human clinical trials. In clinical trials, doctors will collect information from 20-80 volunteers for $\sim 1$ year, on the dose at which the drug is effective, how it is metabolized, absorbed, distributed, excreted, and at which dose side effects are developed (phase I). In phase II clinical trials, minimum and maximum doses are identified in 100-300 volunteers for the following $\sim 2$ years, to assess the drug's effectiveness for a specific cancer. In phase III clinical trials, it will be assessed how well the new drug works compared to standard treatment and the potential adverse effects it may have in 1000-3000 volunteers for $\sim 3$ years. At this stage, the Food and Drug Administration (FDA) will review the data available for the drug ( 2-3 years) and if the drug is effective, safe, and works better that standard of care, the FDA will grant approval for marketing. However, there is a last phase (phase IV) where long-term effects of the drug are assessed over the course of the years. This means that from the time that he drug is discovered until it is up in the pharmaceutical shelves where it is sold, it takes $\sim 12$ 14 years and a considerable amount of medical, scientific, and economical (in the order of billions of dollars) resources together with a substantial quantity of patient volunteers. Despite careful designing and preclinical efforts, only about 5/5000 drugs proceed to clinical trials, and $1 / 5$ of the drugs that do proceed, actually make it to the market (www.medicinenet.com).

The cost of health care for cancer patients is increasing not only due to inflation but also due to an increment in the number of procedures, cases, and the ageing of the population. Because cancer therapies, especially those involving stem cell 
transplantations and palliative chemotherapy in NSCLC for example, are resource intensive, investors in cancer research (such as pharmaceutical companies and individuals who donate to the cause), and cancer patients who pay for the treatment, want to see the benefit of additional procedures and drugs, thus placing constraints on the expenditures. There is therefore a great need to improve decision-making to improve cost-effectiveness of tested treatments, and suggested treatments must show benefit versus current standard of care treatments. To meet this end, in this thesis we have suggested to use a new triplet combination consisting of standard of care treatment modalities (chemotherapeutic/targeted agents and radiation), from which the dose that is effective to reduce tumor burden with minimal side effects is known, with a small molecule inhibitor against $\gamma$-secretase thus preventing NOTCH protein activation. Despite the great availability of different efficient NOTCH inhibitors, there are none that are FDA-approved. However, several inhibitors have been tested in human clinical trials and side effects are known. This prior information would greatly facilitate further designing and development of clinical trials, reducing costs, and speedy implementation in the clinic in case of superior outcome to that of standard of care. There is a plethora of treatment options that are efficacious for the target they were designed for, however, due to a suboptimal clinical trial design, the complete efficacy of the drug upon the tumor burden has not been accurately evaluated. A clear example of this are the NOTCH inhibitors, which have not been successful in reducing significantly tumor burden when delivered as a monotherapy on an unstratified patient population. Our study gives evidence that when NOTCH inhibition treatment is delivered in NOTCH-expressing NSCLC or GBM multicellular spheroids in vitro, and in ectopic xenografts in vivo, both NSCLC and GBM growth is delayed, an effect that is further potentiated in combination with chemoradiation. Further studies are needed to substantiate our findings, but the results so far are encouraging. Moreover, it is imperative to identify companion biomarkers to assess, prospectively, the efficacy and or toxicity of the drug over the course of the treatment, enabling therapeutic regimen redesigning if need be. Here we suggest the use of TLSP as a potential companion biomarker, and although its validation still needs some more time and resources, money in the discovery of companion biomarkers would be well spent since they can aid tremendously in therapeutic assessment, and potentially reduce the amount of trials needed.

\section{Innovation}

The concept of combining chemotherapy or radiation with NOTCH inhibitors is not new, there are several ongoing clinical studies in different cancer types, 
hematological and solid tumors including lung, breast, pancreas, colorectal, and brain cancers. However, what is novel is the triple combination of chemoradiation with NOTCH inhibitors, which is what we propose in both NSCLC and GBM models.

In order to obtain optimal clinical translation, the most physiologically relevant models and clinically sound treatment options must be incorporated in preclinical studies. Our lab has invested a great amount of resources in obtaining more reliable models. The use of 3D cancer spheroid models to test the efficiency of treatment combinations in long-term in vitro is slowly starting to supersede the more common $2 \mathrm{D}$ assays which monitor efficacy in short-term assays. In vivo, the use of orthotopic models and the application of treatment planning software (SmART-Plan) to precisely deliver image-guided radiotherapy using cone beams that can go as low as $1-\mathrm{mm}$ in precision, are novel models and technologies that more closely mimic the clinical setting compared to the ectopic xenografts models which are more widely used.

\section{Market Opportunities}

Current standard of care treatments for both advanced stage NSCLC and first line treatment for GBM, both consisting in a combination of chemotherapeutics/targeted agents and radiation, are giving rise to tumor recurrence. Therefore, there is a need to carefully and thoughtfully design treatment strategies for such patients to improve prognosis and prolong survival. In this thesis we have shown evidence that NOTCHbased treatments are promising in such a respect even though there are still further preclinical studies to be addressed before considering progression into clinical trials such as addressing the effect of the triple combination (chemoradiation and NOTCH inhibition) in orthotopic models for NSCLC, and assessing the effect of the triple combination in immune proficient mice for both models. The observation that the triple therapy (chemoradiation and $\mathrm{NOTCH}$ inhibition) was more effective than chemoradiation for several chemotherapeutic agents and targeted agents in NSCLC and in GBM, is suggestive that other cancers with active NOTCH signaling may benefit of such treatments as well.

NOTCH activating mutations occur in $~ 50 \%$ of T-ALL tumors, however in solid tumors the mutation rate is much lower. In this thesis we have shown that although tumors with NOTCH overexpression are more responsive to NOTCH inhibition therapy, NSCLC tumors with wildtype levels of NOTCH signaling activation, also show significant tumor growth delay with respect to placebo. This suggests that a greater patient population could benefit from the proposed regimen approach. 
In conclusion, if patients that are susceptible to NOTCH inhibition therapy are recognized, companion biomarkers are identified, and the effect of the immune system potentiates, or at least is not detrimental, for the treatment strategy here proposed, it is highly likely that hematological and solid tumors receiving chemotherapeutic, targeted agent and/or radiation treatment will benefit from the addition of NOTCH-based therapies to prolong survival in cancer patients. 


\section{Acknowledgements}



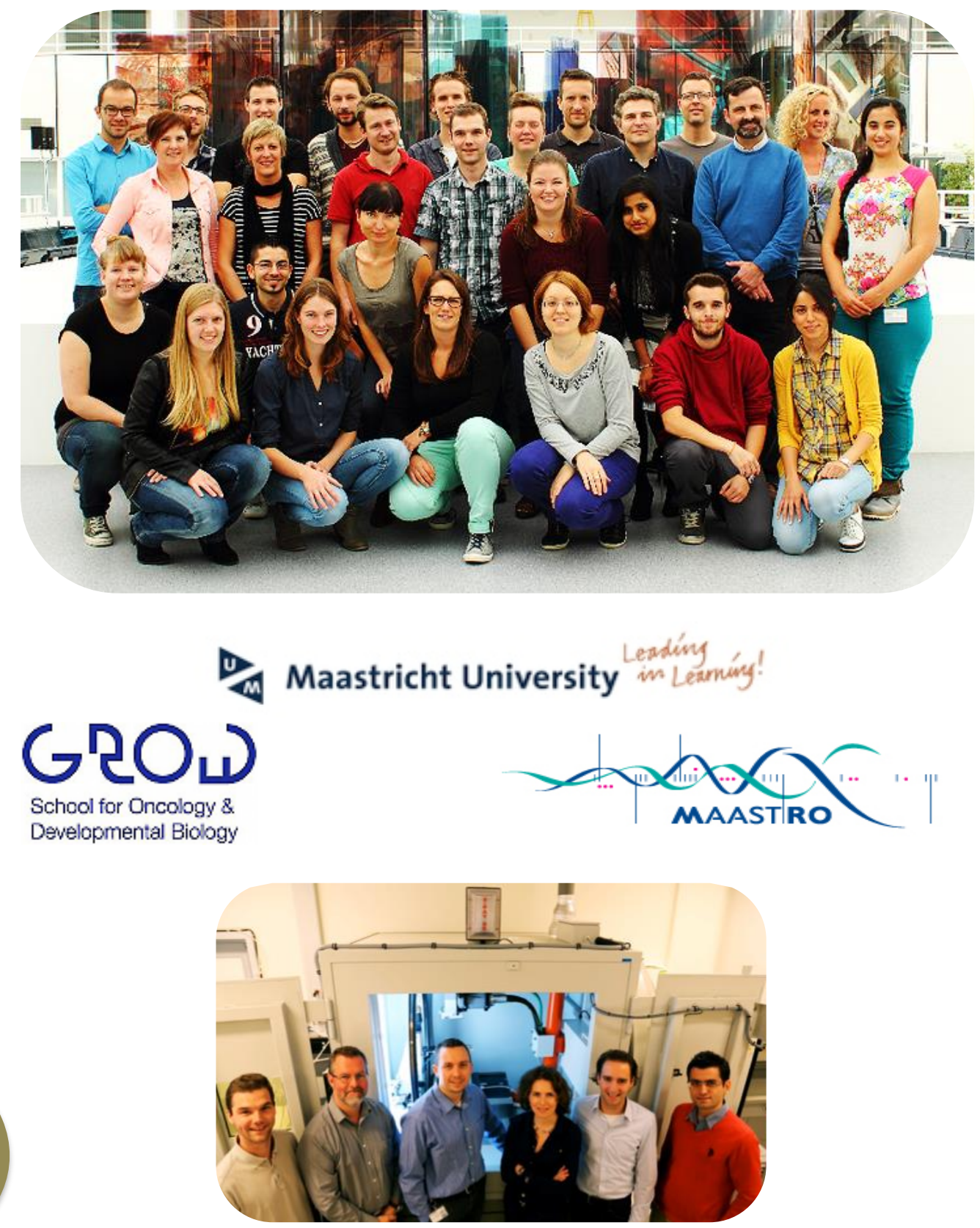

\section{worldwide}

cancer research formerly known as $A K R$

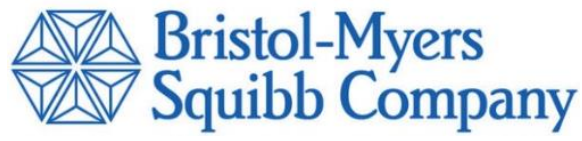




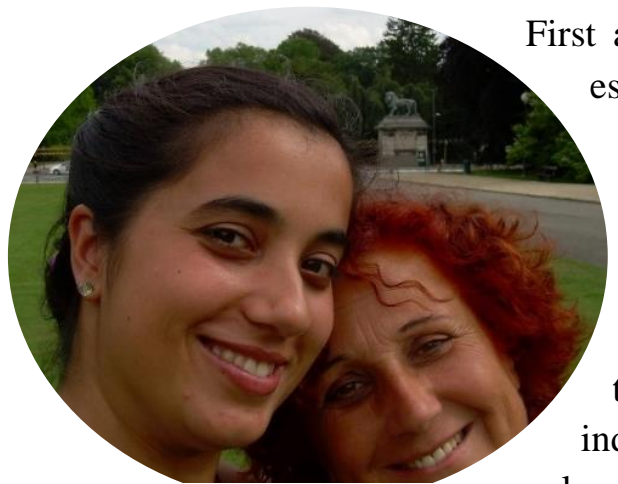

First and foremost, I am grateful to my family, especially to my mom Conchita, and to my belated dad Manolo who recently lost the battle to duodenal cancer. They both not only paid and granted a trilingual (Spanish, English, and French) education, but they never faltered in believing in my potential to become a successful and good-hearted individual. My parents, grandparents, aunts, uncles and cousins

the courage to fight in the face of adversity, to stand up after a fall, and to strive to be a loving, honest, respectful, and forgiving person. As the roman poet Virgil once said: "love conquers all". Their unconditional love, support, and motivation have helped me overcome the obstacles that life has thrown my way and have sculptured me into the woman I am today.

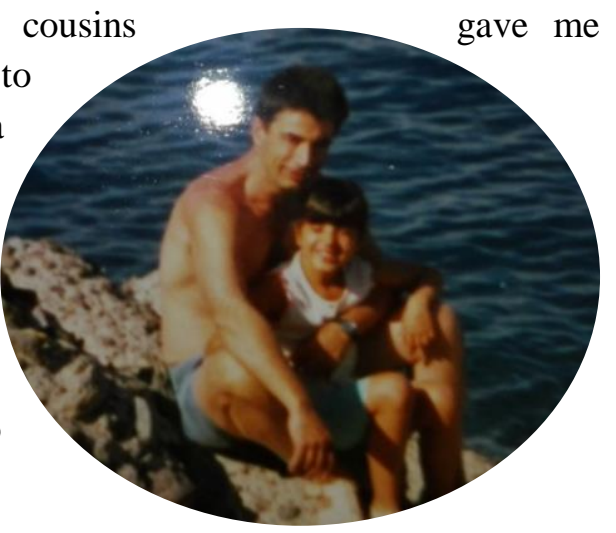

I remember the day I decided to become a research scientist. I was in high school and a young man came to our class to offer us career advice and try to convince us to enroll in the University of Valencia in Spain. I remember I was torn between humanities and sciences, and my career interests were very broad. I wanted to be a doctor, a lawyer, a teacher, an interpreter for the United Nations, a psychologist...I wanted to do charity work for Amnesty International, Doctors without Borders, join Greenpeace... I soon realized that the coincidental root of these career choices was that I wanted to help those in need. The young career advisor started to talk about how the University of Valencia was a pioneer in Spain in introducing Biotechnology as a degree, a degree that would enable us to become research scientists. Research scientists specialized in the medical field could help understand the biology of diseases to ultimately improve treatment regimens for patients. I immediately felt the connection with the career so I completed my Biotechnology degree and my Master degree in Biomedicine, both in Catalonia, Spain. I spent my summer holidays during my degree doing research internships not only in Catalonia, but also in Taiwan and the Czech Republic. I felt like fish in the water in the lab. I enjoyed not only learning to work with bacteria, yeast, zebrafish, and human samples to better understand the complexity of signaling pathways, the process of senescence, breast cancer biology, 
and the potential implications of microRNA in human disorders and therapeutics; but also, in collaborating as a unified team: from technicians, to $\mathrm{PhD}$ students, to eminent professors; with people from different ethnicities, cultures, religions, personalities, and specialized in diverse disciplines. We often hail individuals that have striven for and accomplished success, however we sometimes neglect to acknowledge that scientific triumphs come through collaboration. This is why my second acknowledgment goes to that young male career advisor who gave me direction and focus, and to all my teachers, from kindergarten to Master's degree, who instilled the passion for science, the eagerness to learn, the logic in thinking, the importance of sportsmanship in competition, and the joys of team work.

The process of application to funded $\mathrm{PhD}$ research positions in oncology took several months. Several self-financed offers were granted in Spain and in the United Kingdom (UK) in oncology, and a fellowship was offered for a $\mathrm{PhD}$ position to work in malaria in the UK. Having lost my emotionally-closest grandpa Benigno Iglesias to colorectal cancer when I was 11, and several close family friends to other cancers, I knew my research calling lied in oncology. During my wait, I gained further experience volunteering in the elementary school were my mom worked in, as her teacher assistant, and in her holidays we both hiked the trail of the "Tour of Mont Blanc" which started in Chamonix (France), crossed Switzerland, and ended in Italy. It was on the last day of the tour, already in Italy, when hiking was impeded by a heavy snow storm, that my patience was rewarded: I read a letter from Mrs. Carla Moonen, secretary to my soon-to-be boss, guide, and mentor Professor Marc Vooijs, scheduling an interview for a $\mathrm{PhD}$ position in lung cancer. After passing through the skype call interview phase, our first meeting in person was the day before my official interview, when I also had the pleasure of meeting Mascha Vooijs, Professor Marc Vooijs' wife, and they both gave me directions to Universiteitssingel 50, where my interview would be held the next day. Professor Marc Vooijs' official call of acceptance came some days later and brought tears of joy to my eyes, because I had finally accomplished my goal. On this day, my $\mathrm{PhD}$ path started under his supervision, and it is to him, and to the grant received from Worldwide Cancer Research, that I owe a great big "THANK YOU!" for the financial support to fulfill this opportunity. In addition, to Bristol-Myers Squibb for granting us the NOTCH inhibitor BMS906024 for free to test in our lab, to the University of Maastricht and all its workers, especially Professor Frans Ramaekers and Dr Ton Hopman for monitoring my $\mathrm{PhD}$ progress and personal well-being, and to Patrick Janssen and his secretary Martine Berghof, for their assistance in arranging the documents for me to become an UM-medeworker, and for their welcoming nature. 
Several people have contributed, directly or indirectly, to the practical realization of this $\mathrm{PhD}$ thesis and to all I extend my sincere words of gratitude:

\section{To my promoting team:}

Firstly, I am extremely grateful to my PhD promotor, Professor Marc Vooijs, head

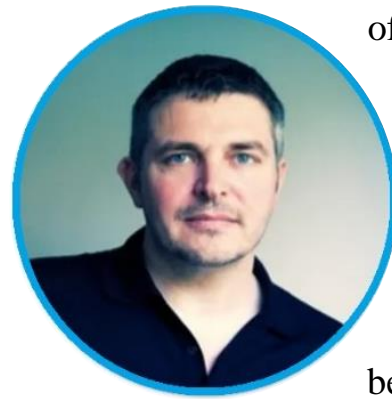
of MaastRO Lab, who believed in my potential from the start, gave me guidance to focus my research and to deepen my knowledge with scientific articles, spoke his mind directly, taught me to be scientifically critical, provided me with resources and connections to make me fruitful in science, instigated me to join scientific conferences to present my work, gave me freedom to investigate and to become independent at work, and was patient with me when my father died on my last year of $\mathrm{PhD}$.

Secondly, I am very grateful to my co-promoters, Assistant Professor Jan Theys and Associate Professor Ludwig Dubois. They were not only my daily supervisors at work and believed in my potential as a researcher, but they also cared for me to have a balanced life outside of work, making sure I took care of myself, did other activities, and didn't overwork.

Jan, you were like a daddy lion who protected me, and our work, whenever it was criticized in meetings. Thanks to your care when I came to the Netherlands by helping me check out my would-be apartment in the city center, and to the Western Union worker who advised me against transferring money for an apartment before seeing it, I was not scammed when I arrived to the Netherlands. Jan, you were always there to give me a positive outlook on our data, which was a great balance for me since I am already quite

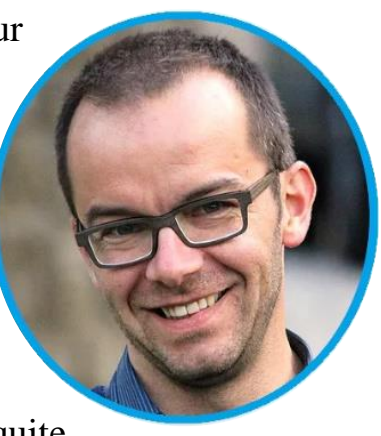
auto-critical. Despite our initial challenges, I really value your input as a supervisor, always downscaling my experiments and helping me focus my multiple interests into a concrete direction. Jan, you were a great model of written and oral communicator given that you certainly have that proverbial gift of gab. 


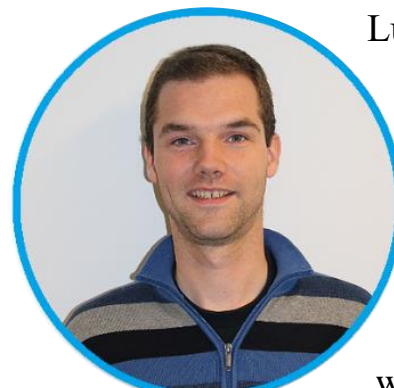

Ludwig, you were my "go-to" mentor at whichever day, time, and place. You always made time, despite your busy schedule, to hear me out, solve my doubts, to help me irradiate mice late at night, and gave me advice on animal experiments and statistics. You were always diligent in answering rapidly all my emails and correcting my work. Thank you for your patience, and showing me tough love with your criticism, which helped me become a better scientist. I will always remember your evening signs of affection saying: "Venus, are you going to the gym today? Go home girl!”.

Thank you both for all your support and concern for my well-being during my daddy's illness and when he eventually passed away.

To Prof Philippe Lambin, thank you for the scientific input from a pathologist's perspective during Refereer avonds/lunches in the clinic, for the financial and radiotherapeutic instrumental support for this project.

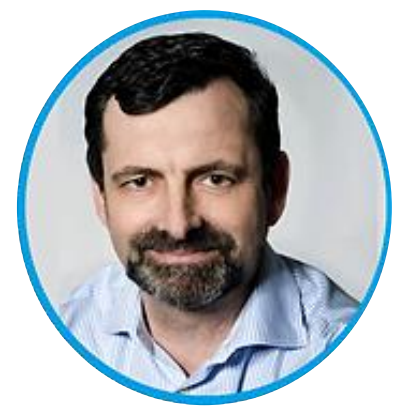

\section{To my assessment committee:}

To the chairman Prof Dirk de Ruysscher, MD, PhD; and to Prof Frans Ramaekers, $\mathrm{PhD}$; Prof. Boris Kramer, MD, PhD; Prof. Jan Bussink, MD, PhD; and Dr Pierre Close, $\mathrm{PhD}$; who spared time from their busy schedules and put effort to read and make valuable remarks of my thesis work.

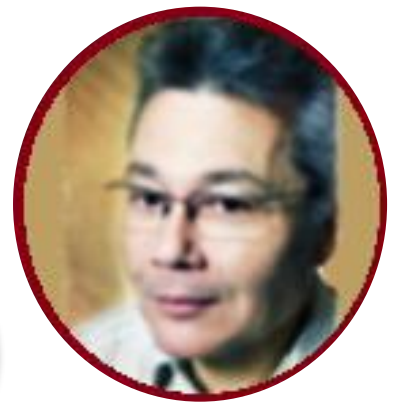

To Prof Dr. Dirk De Ruysscher, thank you for the one-toone meetings giving me advice on how best to analyze and approach my orthotopic lung cancer study, for the medical input on my manuscripts, and the inspiring awareness on how to make my study more clinically relevant. 


\section{To my fellow NOTCH group colleagues:}

To Dr Arjan Groot, the great scientific enthusiast! Thank you for spreading your positive eagerness to have the famous "killer experiments", for sharing your past and present life anecdotes, for giving me conversation in English to make me feel welcome and integrated in the group, and for giving me your help when carrying out experiments, especially during my first year and when creating new cell lines for our study. Thank you for your support throughout this $\mathrm{PhD}$ process.
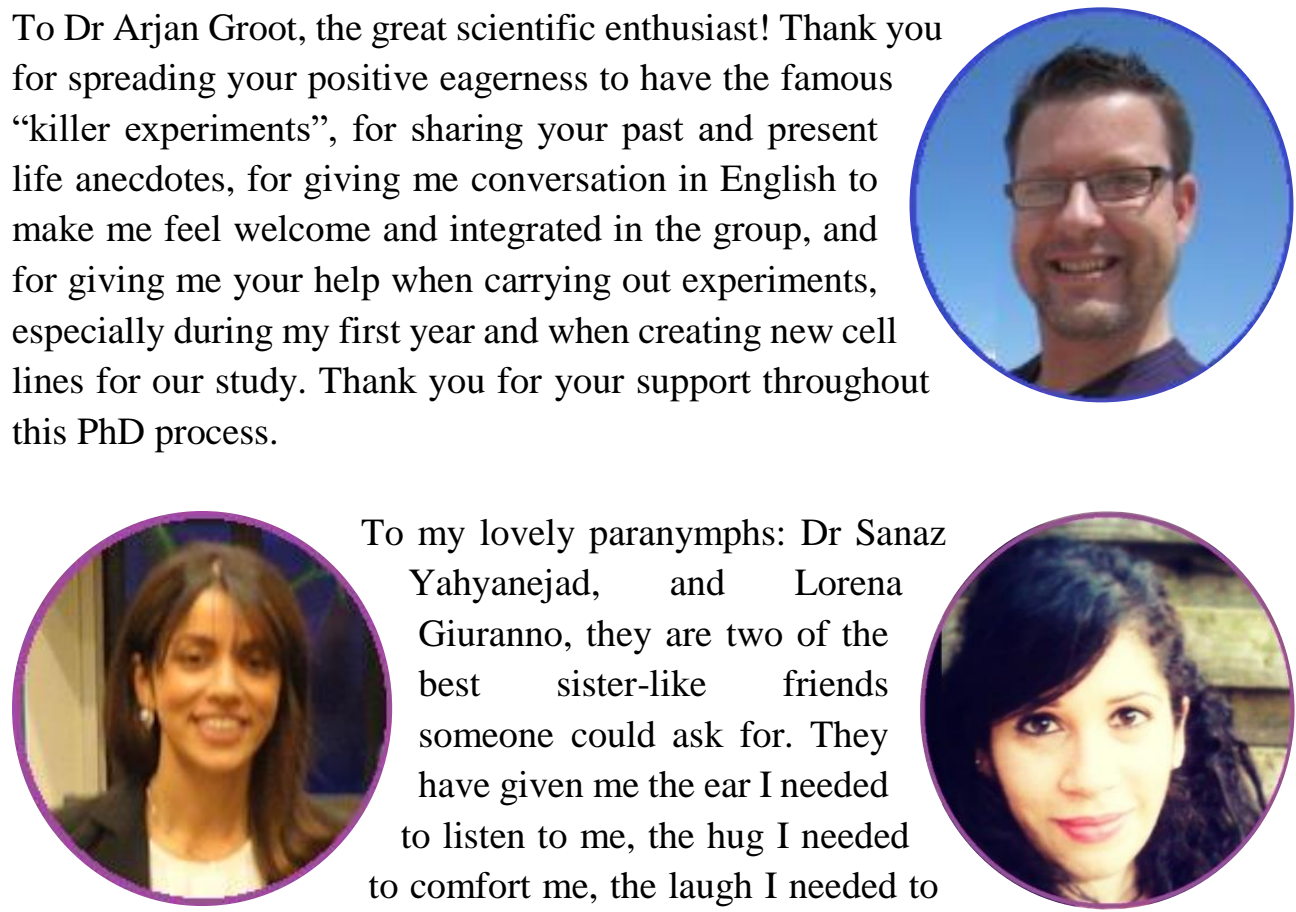
brighten my day, the company I needed so I wouldn't feel lonely, the affection I needed to feel cared for, and the advice I needed in times of doubt. Thank you for the company during our late-night work in the lab, our chats about life anecdotes and science, sharing home-cooked food, laughs and games. You both have beautiful, loving, caring, and colorful souls and I will always remember and carry you in my heart. Despite the time and the distance, I know we will be there for each other. Love you tons!!

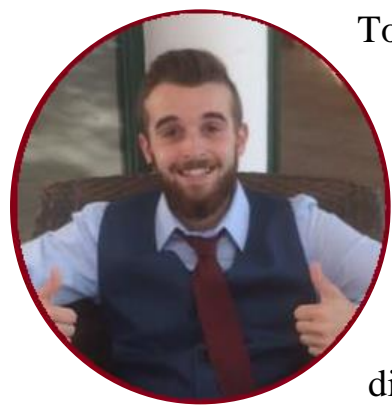

To my fellow Spanish friend Eloy Moreno Roig, Ilos!, the joy of the party, the extremely sociable buddy, the person who wouldn't miss an opportunity to crack a joke, and the relentless match-maker. Thank you for your interest and advices for my work, for not forgetting about me in your activity planning and consistently inviting me over for a dinner night with friends and new acquaintances from different backgrounds, perspectives of life and how to live it. Thank you for the Dixit-playing nights, the nights of cooking new dishes, watching Game of Thrones, skiing, dancing, or going to the gym together. Thank you for 
bringing more fun and diversity to my life, for the nice company, the understanding, and keeping in contact with me in my time of need. I hope life keeps bringing a smile to your face. Lots of hugs my dear.

To my friend Jonathan Ient, our dear Joffrey, who always was ready to listen to my venting without complaining, who brought useful advices to the table, and has a sweet, patient, and understanding nature. Thank you for the laidback and agreeable moments going out or staying in with friends and enjoying genuine laughs and pleasant conversations. I hope you are successful in your endeavors. A great big hug.

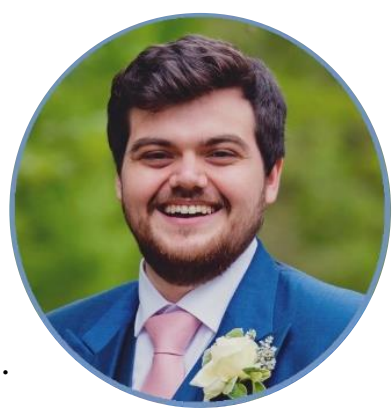

To Dr Carolien Wansleeben, thank you for our nice meetings eating out sushi,

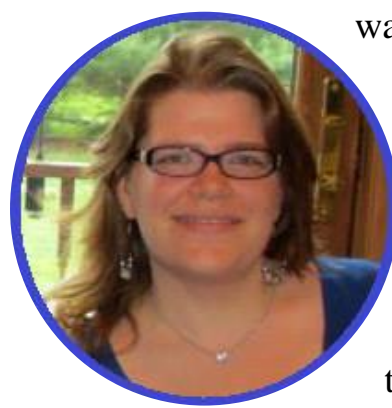
watching a movie at your place discussing about life experiences and future plans, or skiing in Austria. Your tough love and your work criticism made me aware of how to improve myself. I am very grateful for your help with my experiments, together with Ludwig, when I had to go unexpectedly back home to attend my dad's surgery and be there for his recovery. You gave me the opportunity to have some extra time with him and I am very thankful for that. I do wish that you are able to find a group where you feel like at home and a job that you are cheerful to go to.

To Dr Paolo Rusconi, dear Paolino!!, thank you for your calm and chill attitude about life which made me want to stop and relax once in a while, thank you for being an example of meticulous worker and article perusal enthusiast, for your encouraging words when we first started skiing that helped me overcome my fears, and for the nice chats during shared dinners out with friends where you shared your lovely and naughty laugh with us. All the best for your scientific future in the company.

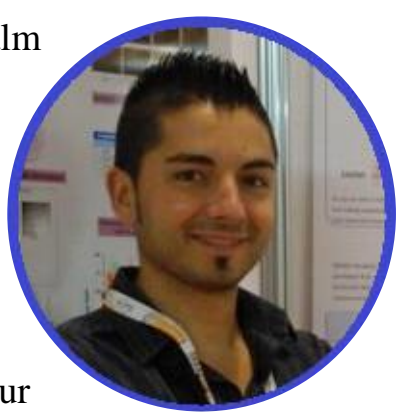




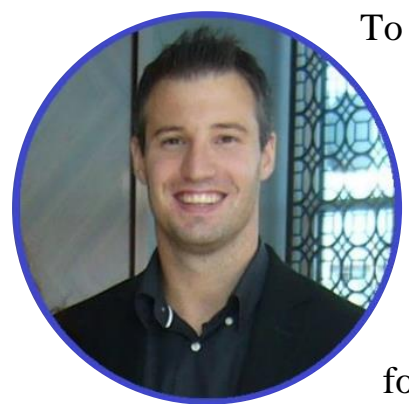

To Dr Roger Habets, my roomie during my first years, you were always kind and friendly with me, gave me supporting and encouraging words, translated to English the Limburg-Dutch conversations so that I felt included, and shared interesting chats about life and science.

positive attitude, and your extroversive and welcoming nature. Thank you also for your constant signs of appreciation for the food I shared with you. I hope you can find a job that potentiates these beautiful qualities making you feel comfortable and in harmony with yourself. Hugs!
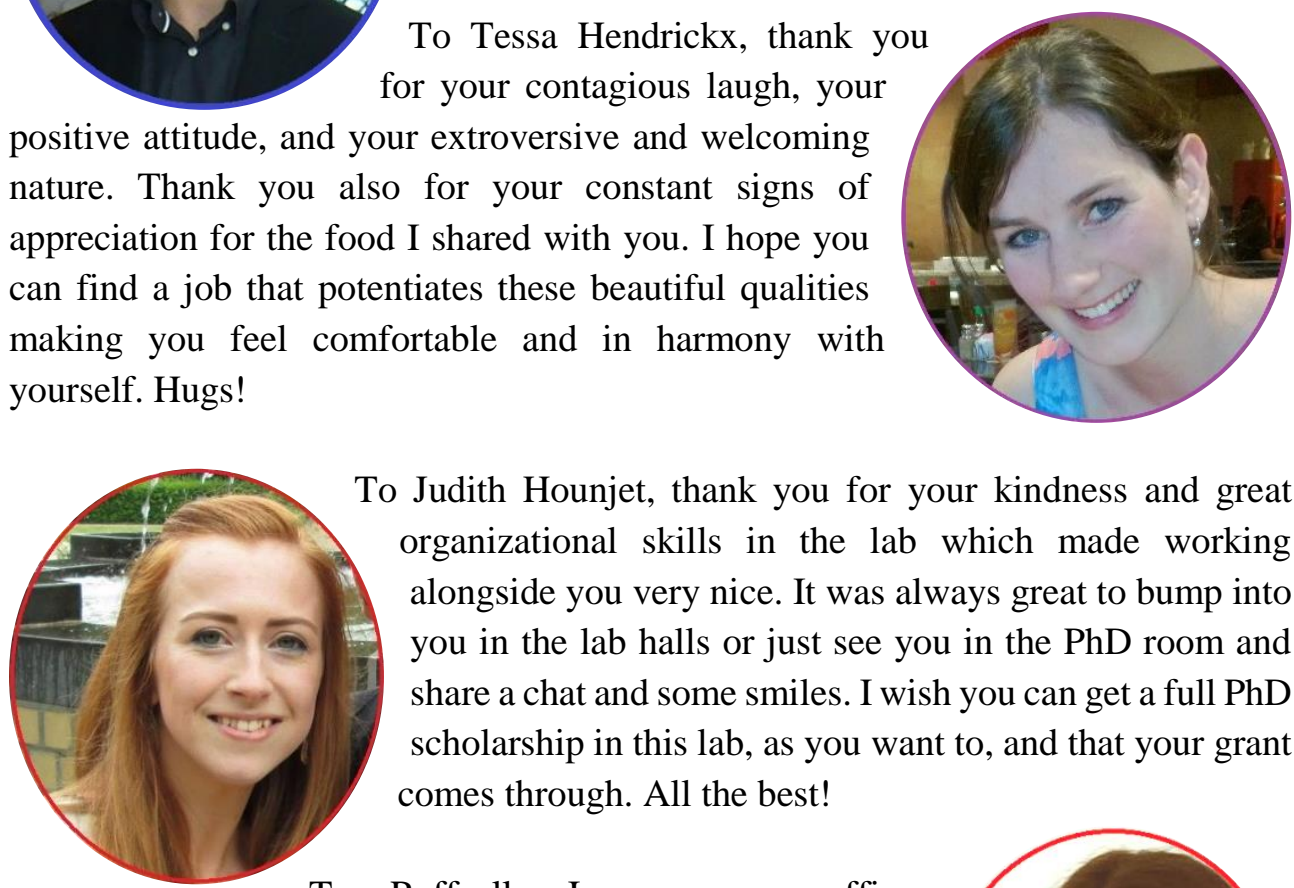

To Judith Hounjet, thank you for your kindness and great organizational skills in the lab which made working alongside you very nice. It was always great to bump into you in the lab halls or just see you in the $\mathrm{PhD}$ room and share a chat and some smiles. I wish you can get a full $\mathrm{PhD}$ scholarship in this lab, as you want to, and that your grant comes through. All the best!

To Raffaella Iannone, my office roomie, thank you for the happy conversations about science and life, for the great time at ESTRO in Brussels and the post-conference roaming around enjoying the sites and eating Belgian chocolates, and for the gatherings after work to enjoy some home-cooked meal together with some friends. I hope you enjoy your married life and your baby

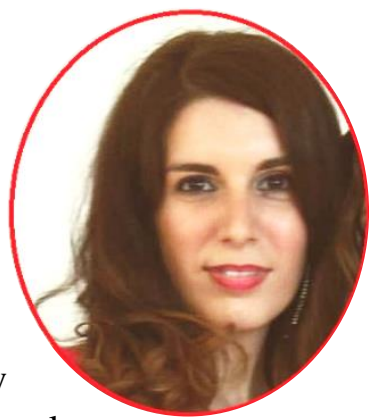
Josephine. All my best wishes for your lovely

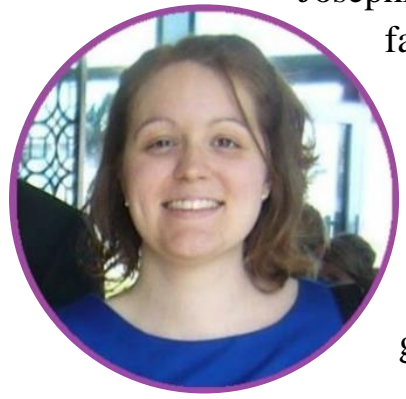
family.

To Lydie Barbeau, thank you for sharing chats, dinners and get-togethers during my first years. I very much appreciate your detail-oriented demeanor when visiting my home and for birthdays, your help, hard work, and great organization skills throughout my $\mathrm{PhD}$, especially 
when I went on conferences or back home for my dad's surgeries. Thank you for the fabulous time in Istanbul for Sanaz's wedding, planning site-seeing excursions and chatting. All my best wishes for your future job.

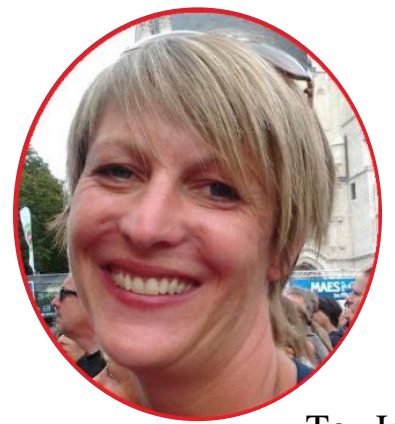

To Kim Paesmans, thank you for your experimental assistance, your patience, your lovely greeting smile, and your delicious speculaaskoekjes for Christmas. I hope you are happy in your new job. Hugs!

To Jolanda Piepers, thank you for being comprehensive and compassionate, for your positive and realistic attitude, and your advices in meetings.

To the students I helped supervise: Alyssa Lemmens, Marloes

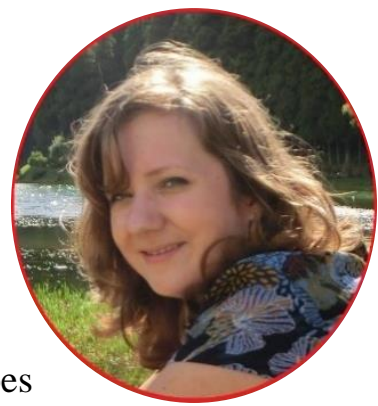
Ijff, Arjan Mofers, and Kenneth Pasmans, thank you for your willingness to learn from me, your contributions to my thesis project, your patience, and for sharing laughs on life anecdotes and science.

\section{To other MaastRO lab members:}

Thank you for the constructive criticism during MLMs, ideas for new research orientations, your willingness to share protocols and expertise.

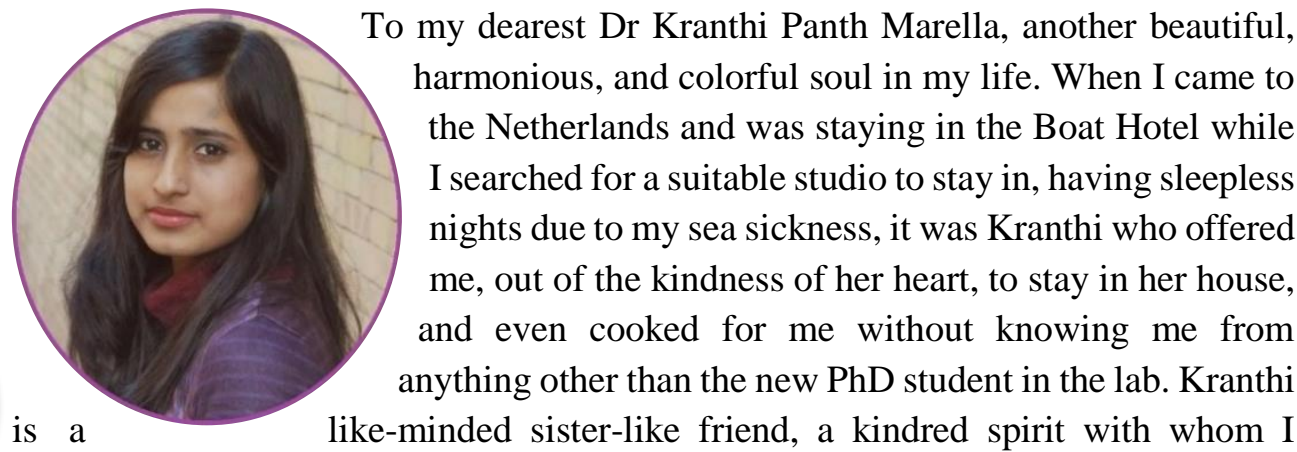
always felt understood, supported, pampered, and loved. Thank you for all the latenight succulent vegetarian dinners, exhilarating conversations and laughs that we shared alone, with more friends, and/or with our respective families. I know that our lives will cross again and we will be there in times of joy and sadness for each other 
despite the distance. Love you very much my dear!! A great big hug for your lovely family, especially to the newest adorable addition, little Ms. sunshine: Sveccha.

To my Latin dancing friend from the Dominican Republic Dr Verónica Olivo Pimentel, thank you for all your warmth, advice, full-day sauna days, sushi eating, dancing out nights, and late-night two-sided free-therapeutic chats until our eyes couldn't remain open any longer. Thank you for being an understanding friend and having my back in times of need. I hope you find that what makes you outmost happy in life in the environment that is best

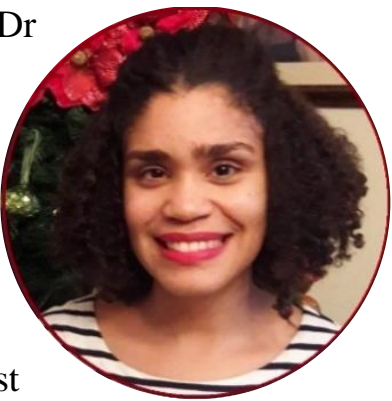
suited for you to succeed. I send you a big warm hug and a great big thank you. Love you tons!

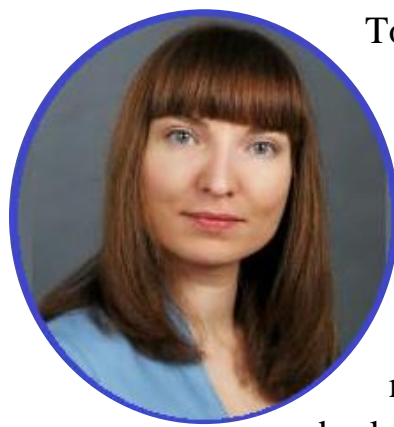

To Dr Ala Yaromina, thank you for your constructive criticism to improve my work, for making me feel welcome in the lab chatting and confiding in me, and for your exemplar predisposition to aid me and offer me advice whenever I had doubts on analysis, stainings or animal experiments. I will always remember your detailoriented demeanor on my first birthday in the lab, one month after my arrival, when you surprised me with a basket with aromatic body cream, soap, and flower petals beautifully decorated, or when you walked with me, together with your husband Georgy and son Gleb, in the solidarity walk for cancer patients and you showed me so much care and consideration for my well-being. Thank you for the beautiful connection we shared throughout my stay in the lab, I always felt I could trust and confide in you. I am very happy that you finally got your well-deserved permanent position at MaastRO. Congratulations! A great big hug.

To Dr Marike van Gisbergen, the relentless compassionate and empathetic friend, my late-night hard-working and sushi-eating buddy (until your allergy manifested), animal lover and protector. I will always be grateful for your acts of kindness when you lent me your extra mattress and blanket when my studio was still unfurnished, or when you offered me fruits in the evenings to revitalize me. Thank you for the scientific input, the

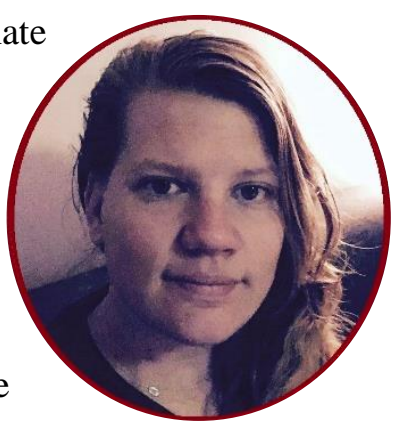


lovely evenings enjoying a movie and our goodie bags on Lady's Night in the Euroscoop, our nights out eating sushi or nights in eating home-made food at my place with Teun, for the jokes, the laughs and your understanding and caring nature. I have no doubt you will be an exceptional mother to your baby girl Lara. You are truly a great person and I am ecstatic to have met you. A great hug to you and your lovely family.

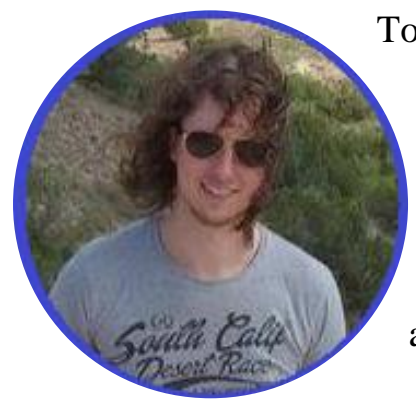

To Raymon Niemans, the chillaxed, easy-going, lively colleague! Thank you for the fooling around imitating Donald Duck, your positive outlook, for our singing duets in the clean tissue culture room which made my day, your appraisal of my home-made food, your scientific input and the chats on holiday escapades. I had a great time with you, thank you!

To Dr Nicolle Rekers, my beautiful charismatic and outgoing friend, the extremely energetic, health-oriented, and seeker of positive vibes. Thank you for the openness in our talks, our Dutch-Spanish language exchange classes, the skiing holidays, the nights out dancing and laughing, or enjoying a home-cooked meal at one of our homes. I hope wherever you are, your lovely smile never fades. A great big hug.
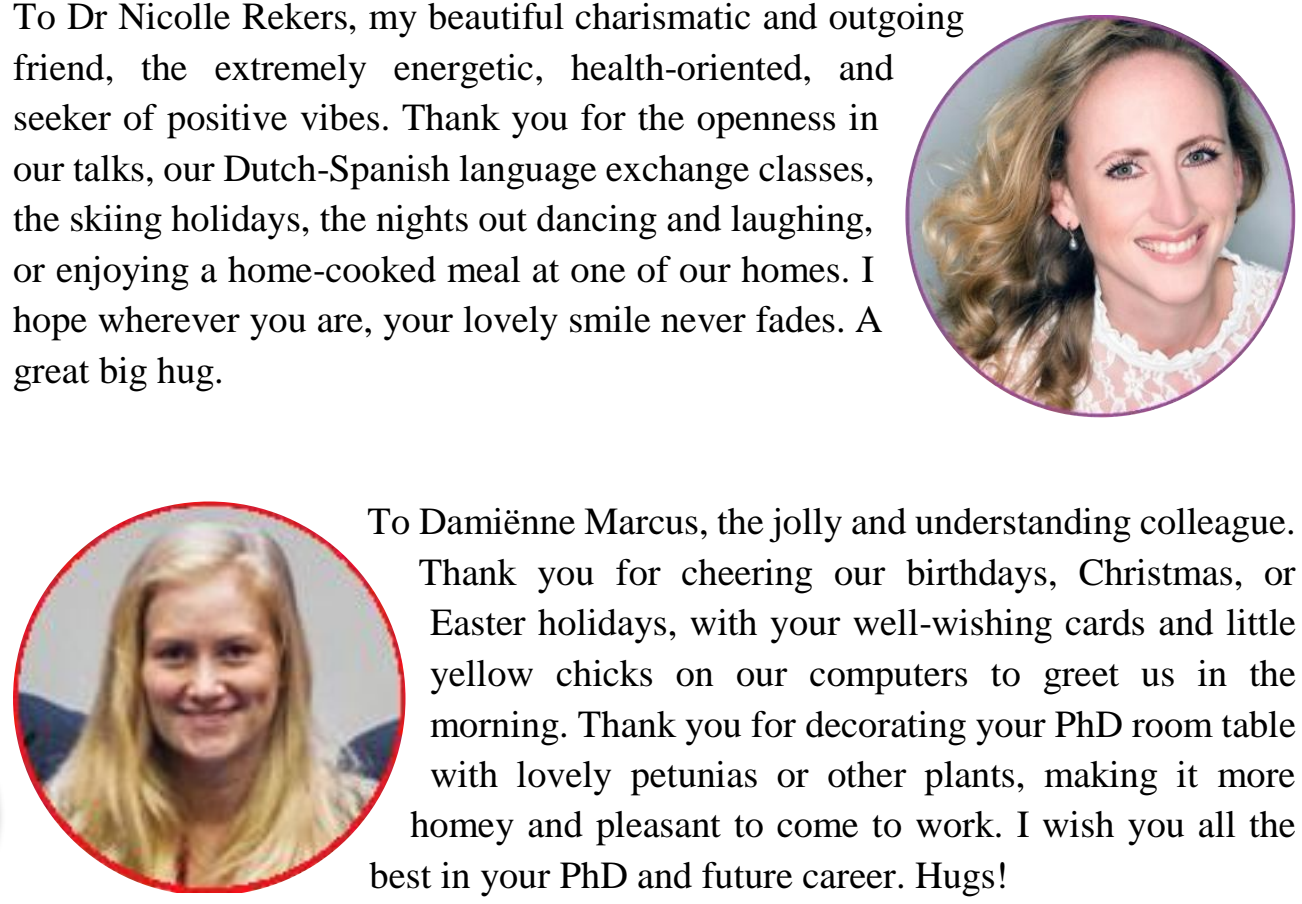
best in your $\mathrm{PhD}$ and future career. Hugs! 
To Dr Nanda Parvathaneni, the calm and easy-going friend, I always felt at ease talking to you, you were always understanding, gave me your vote of confidence, and instilled enthusiasm in me to keep looking forward, offered good advice and positive vision of the future. I hope you have time to enjoy your family with the lovely new addition Mihir and find a rewarding job that makes you cheerful. Thank you for the time we spent together.
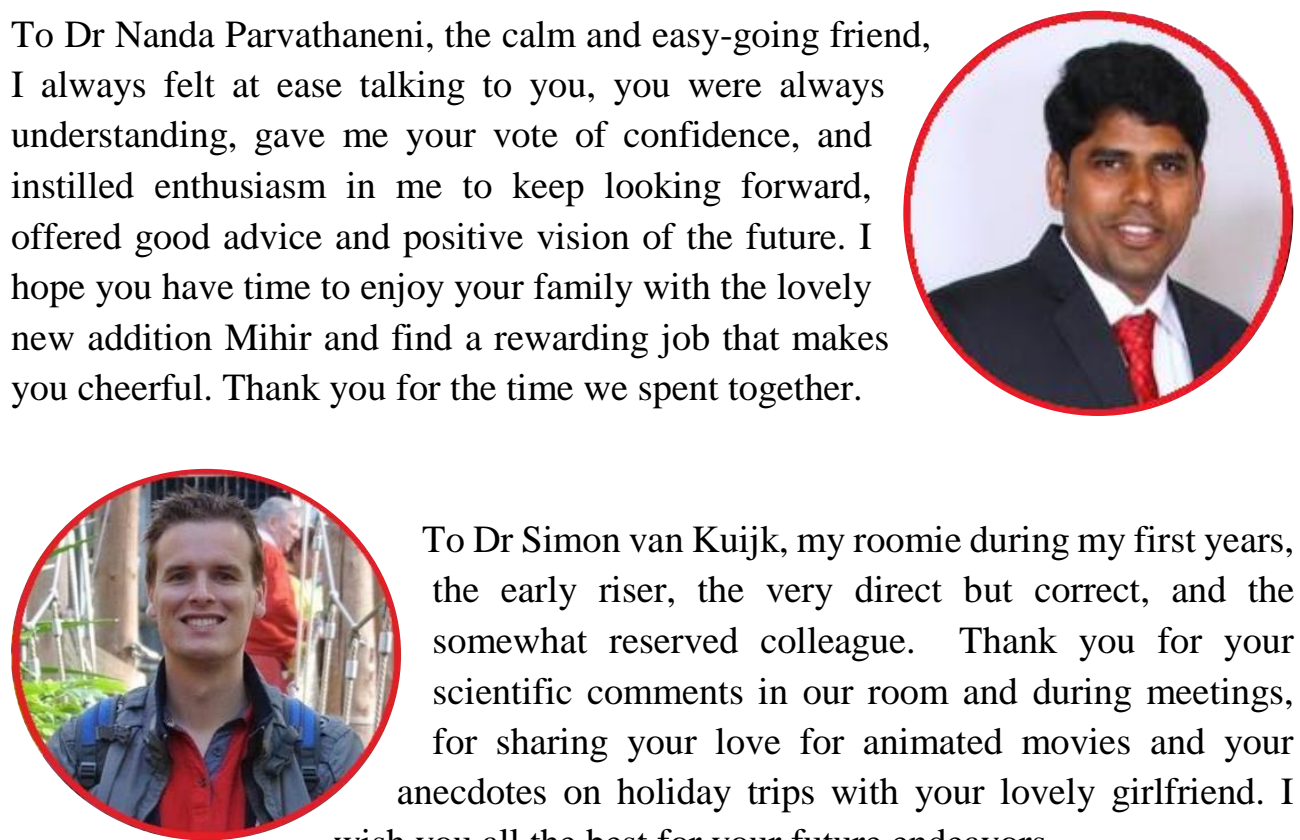

To Dr Simon van Kuijk, my roomie during my first years, the early riser, the very direct but correct, and the somewhat reserved colleague. Thank you for your scientific comments in our room and during meetings, for sharing your love for animated movies and your anecdotes on holiday trips with your lovely girlfriend. I wish you all the best for your future endeavors.

To Dr Sarah Peeters, for being a great colleague, thank you for gifting me your nice smile and laughs, for the nice times in Sanaz's wedding in Istanbul, the teambuilding activities, the lady night's out, and your energetic and positive predisposition to life. I hope you are happy in your future endeavors and continue smiling! Hugs!

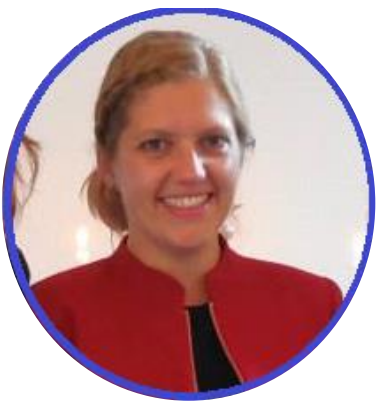

To Dr Linda Spiegelberg, thank you for your lovely, calm and harmonious soul, for

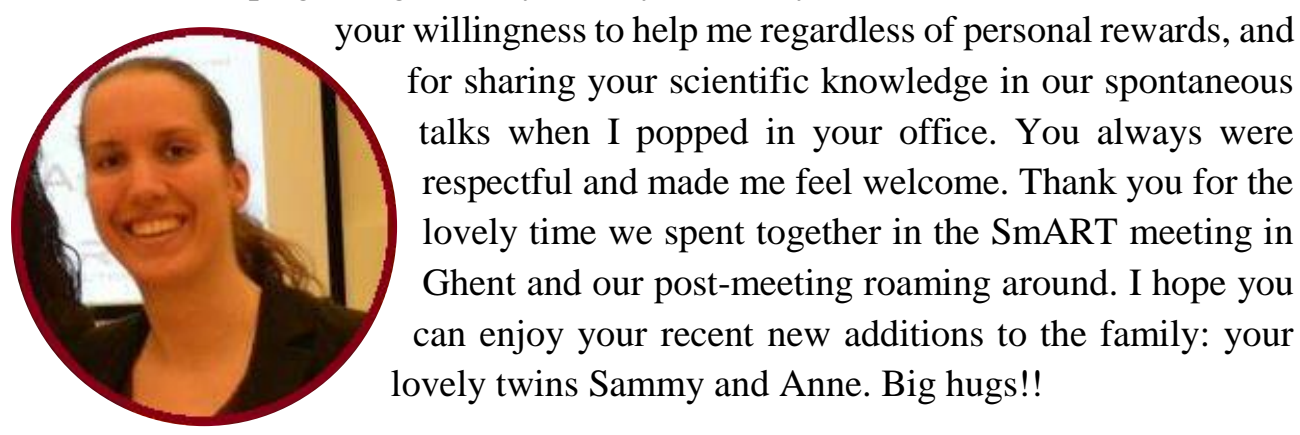




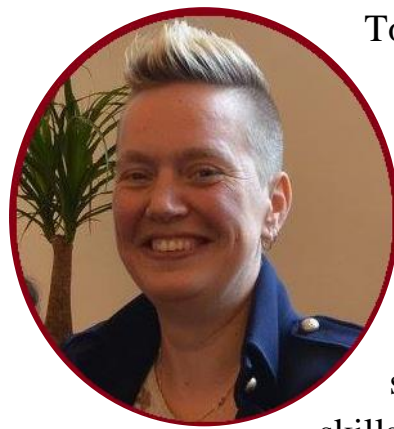

To Natasja Lieuwes, my dear Taschi, thank you for your exceptional organization skills, your creative ideas for teambuilding tasks and for gifts to other lab members, your solidarity speaking in English in my presence to make me feel included in the conversation, your willingness to listen to my venting out in the secretary/technicians' office or in the animal facility, for sharing lovely chats about life, and your great teaching skills in mouse-related work. It was always a pleasure to work with you and learn from you. I had a blast being your partner in crime in the Mol team for teambuilding!

To Rianne Biemans, thank you for your sweetness and kindness. You were always helpful with all the cell line database related things, or really any help I needed at the moment from setting up the UM-internet access in my phone to experiments in the lab or chats about life. You were always cheerful and lent me your ear to listen. Thank you for the lovely times during teambuilding, your energy and for sharing your smile with me. A big hug!
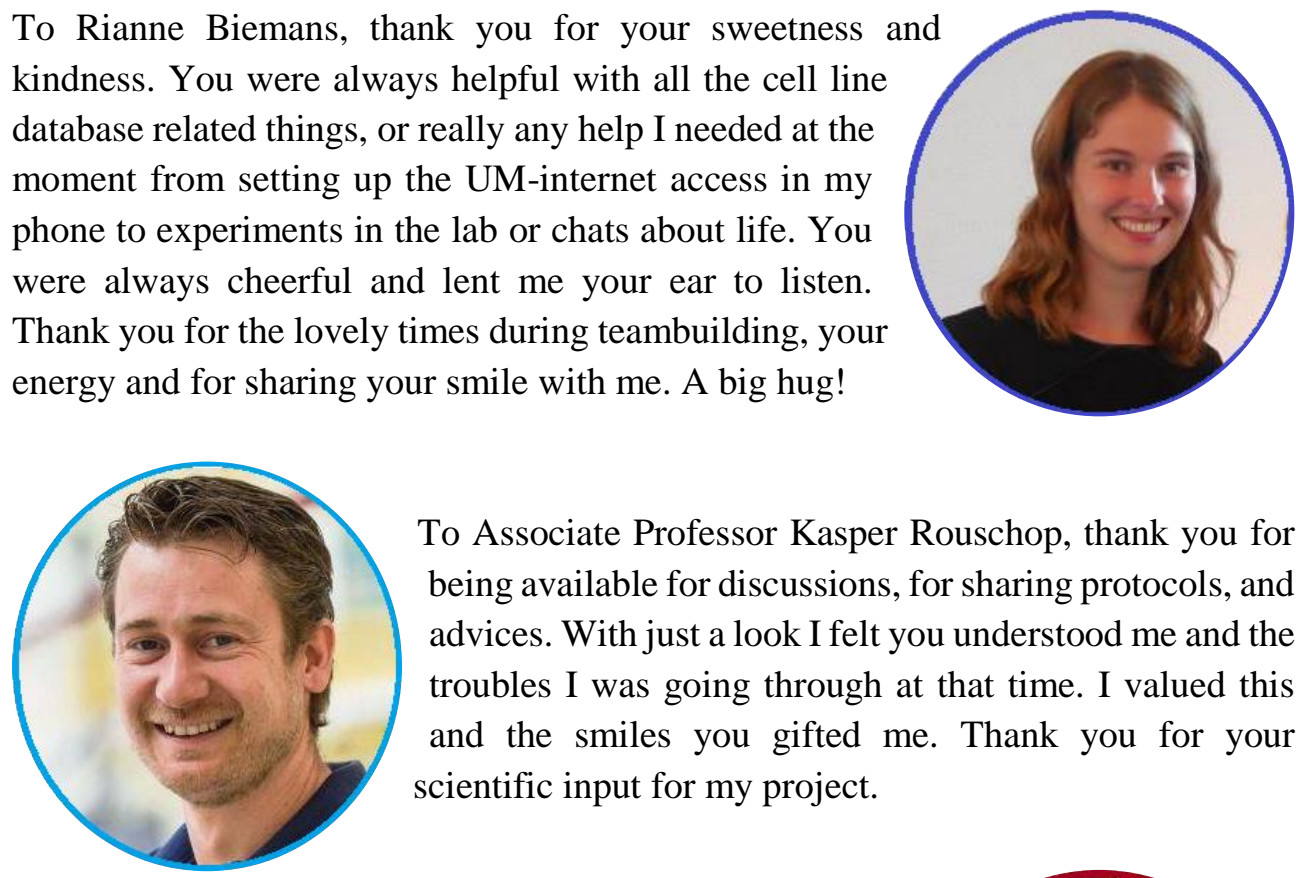

To Associate Professor Kasper Rouschop, thank you for being available for discussions, for sharing protocols, and advices. With just a look I felt you understood me and the troubles I was going through at that time. I valued this and the smiles you gifted me. Thank you for your scientific input for my project.

To Dr Maud de Klerk Starmans, thank you for your statistical advices during my first year. Although we didn't spend much time together, whenever I stepped in your office you were always nice to me. Thank you very much.

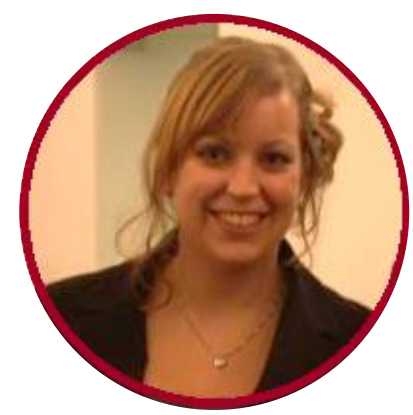




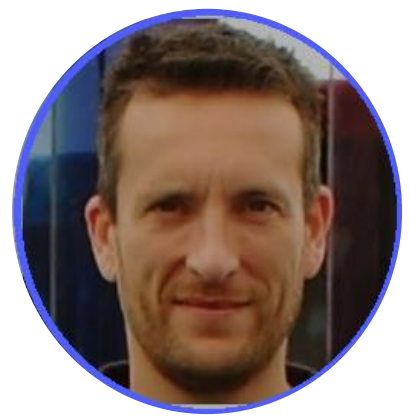

To Dr Barry Jütten, thank you for pushing me to practice Dutch with you and to improve it day-by-day during my first year. Thank you for sharing protocols and computer programs with me, and for your advices during MLM and outside in the hallways.

To Ass Prof Twan van den Beucken, thank you for always being sweet and helpful with me. Whenever I discussed data with you during MLM, in your office, or in conferences, you always had some useful constructive criticism to offer. I always received nice complements, counsel and encouragement from you. Thank you for your kind words.

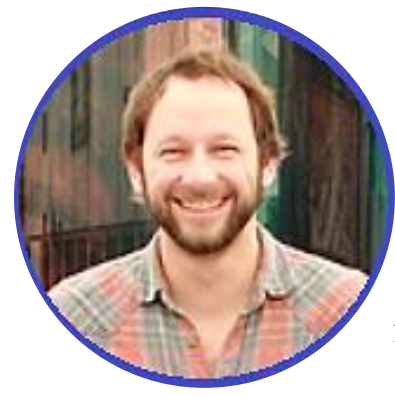

To Tom Keulers, thank you for your hilarious and friendly demeanor, for the enjoyable and relaxing times during the ERRS, Wolfsburg, and the GROW conferences, but also for sharing your scientific knowledge with me. You managed to make me crack a smile even when I wasn't feeling at my best.

To Dr Marco Schaaf, thank for your calm, thoughtful, and amiable attitude, for your good advices on $\mathrm{PhD}$ related issues and your scientific input. I always enjoyed our comforting chats and your company and wish you all the best in future endeavours.

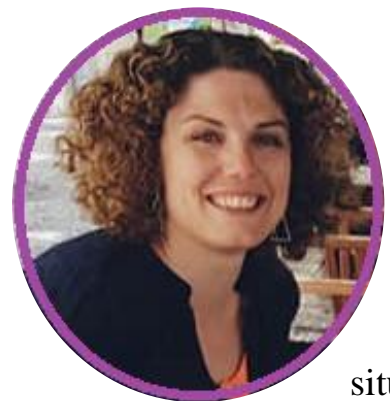

To Dr Marijke Zonneveld, thank you for your lovely energy and your outgoing nature, thank you for the great time skiing in Austria sharing a room, and in the Wolfsburg conference. You always lend me an ear to listen to me and were compassionate and understanding of my situation. 
To Kim Savelkouls, my dear Kimmie, thank you for sharing your love of dancing and singing with me. I enjoyed your company and creative input for all the artistic projects we did for the different lab events, and farewell gifts. Your laugh outbursts were very contagious, I loved them! I always enjoyed your company. Hugs!
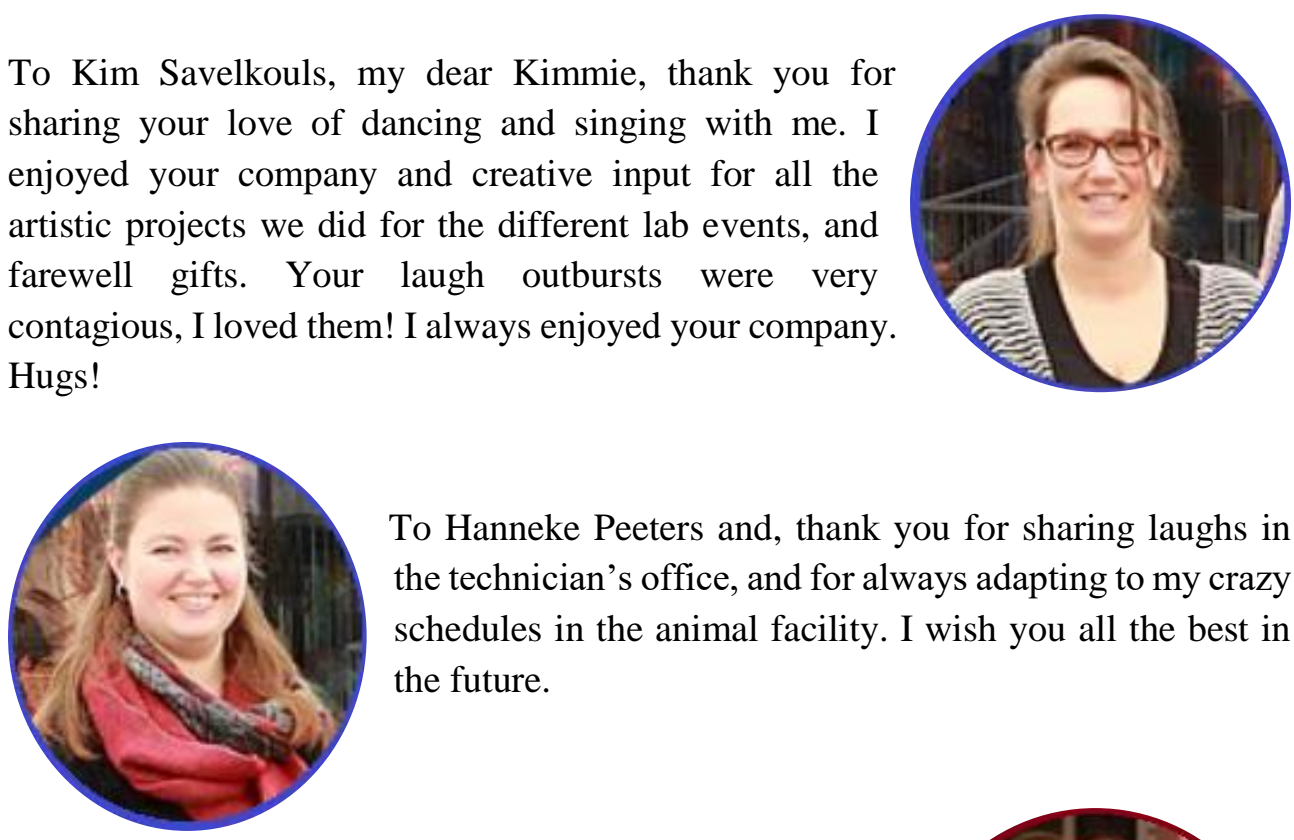

To Hanneke Peeters and, thank you for sharing laughs in the technician's office, and for always adapting to my crazy schedules in the animal facility. I wish you all the best in the future.

To Carla Moonen, thank you for always offering me a helping hand to fill in forms in Dutch, ordering reagents, scheduling meetings, delivering packages/letters, in essence, for whatever was needed at the time. Thank you for your smile when greeting me and your patience.

\section{To MaastRO Clinic members:}

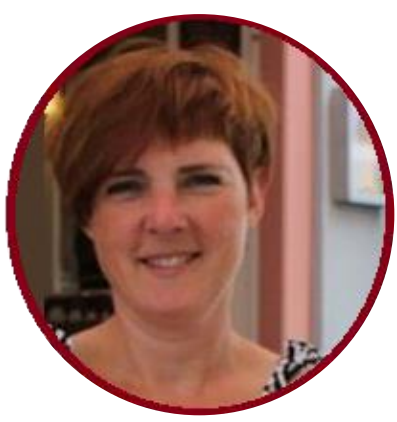

To Prof Frank Verhaegen, thank you so much for all the help and support you gave me for the orthotopic lung cancer model. You were always arranging SmART meetings and enhancing collaboration with me and other
MaastRO lab members and you had a positive outlook and
encouraging attitude for our work. Your input was
invaluable to get this chapter of the thesis going. Thank
you for offering the possibility to attend the SmART
meeting and present the data in the conference with the
possibility to get a publication out of it and inviting me for
dinner with your PhD students. 
To Stefan van Hoof, you were my life savior. Your great approachability and kindness made it very easy and a real pleasure to work with you. You were always there for me whatever day and time I needed you for any SmART-related issues or about our collaborative project, and despite your very busy agenda. You were always critical in your input and had creative ideas to overcome problems. You were also very friendly, trustworthy, and always made me feel welcome. I wish you all the best in your future endeavors.

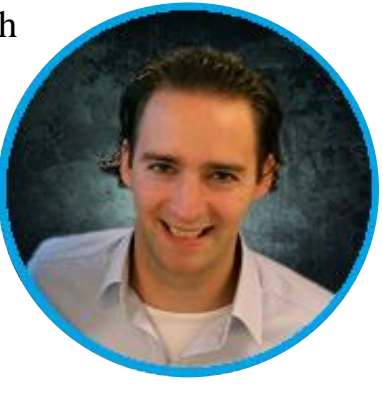

To Dr Patrick Granton, the energetic, out-going, and activity planner. Although I didn't have the pleasure to work with you closely, I did
appreciate all your input for my project and for life. I will
always remember your cheerful predisposition when
sharing chats with me. Thank you for the home cooked
meals at your place and Sanazi's and for always making
me feel welcome at your home and in your life. Thank you
for keeping me in mind to celebrate your lovely wedding
day. I hope our friendship keeps growing throughout the years.

To Ana Vaniqui de Santana and Lotte Schyns, you were both very kind and friendly,

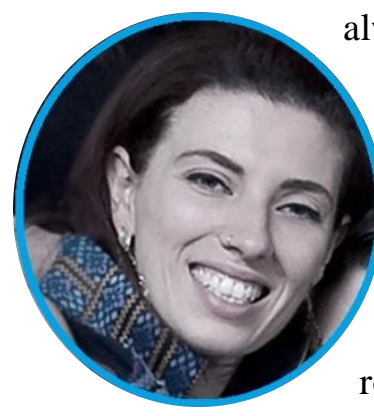
always ready to help and efficient in answering questions and giving me input on the collaborative project we did. It was a pleasure to work with you both. Lotte, I really enjoyed the time we spent in Ghent in the SmART meeting and roaming around the city.

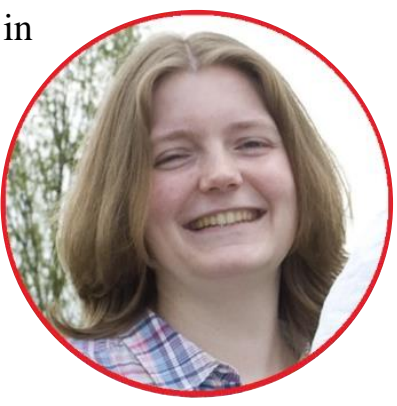


To Relinde Lieverse, thank you for your empathetic nature and your willingness to collaborate and help in whatever was needed, thank you for popping up in my office to share some hot chocolate and chitchat despite your busy schedule. I truly believe you will make a great caring and sympathetic medical doctor one day. A great hug!

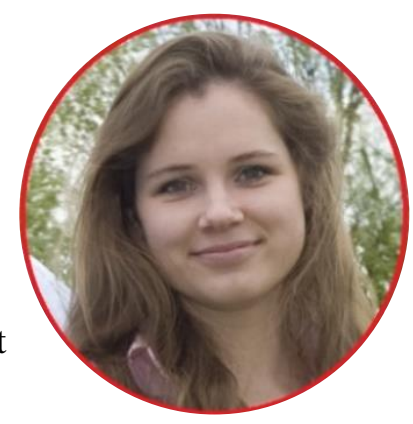

To all other MaastRO Clinic members: Karen Zegers, Aniek Even, Mark Podesta, Ralph Leijenaar, Ruben Larue, Daniela Trani, Jurgen Peerlings, Shane White, Sean Walsh, Evelyn de Jong, Janita van Timmeren, Andre Dekker, Wouter van Elmpt, Lucas Peerson, Ruud Houben, Maaike Berbee, Skadi van der Meer, Sara Botelho de Carvalho, Raghu Van Trichür, Isabel Pinheiro de Almeida, Gabriel Paiva Fonseca, Brent van der Heyden, Imke Demers, Rianne Herben, Lars Murrer ...thank you for the input during Refereeravonds and lunches, for the constructive criticism and the relaxing chats and time after conferences, at Café Thembi or in our annual ski trips.

\section{To all collaborators and co-authors:}

To Dr Jack Cleutjens, Dr Jos Broers, Dr Mario Losen, Dr Ruud Houben, Dr Jan Bussink, Dr Henry King, Jos Prickaerts, Prof Dr Anthony J. Chalmers, Prof Dr Susan C Short, and Dr Marjon J. Gijbels, thank you for your invaluable input and resources, advice and collaboration for this project.

To Saskia Seeldrayers, Richard Frijnts, Clarice Soogelee, Rik Tinnemans, Inger van Rhijn, Nadine Doorn, and Angeli Streefkerk, thank you for facilitating my experimental work with mice, accepting and processing last minute animal orders, making sure animal welfare was closely monitored, and giving me support with experimental procedures like administration of oral gavages or enabling the use of anesthetic equipment for surgical procedures.

To Sandra Zimny and Martine Hulsbosch thank you for your patience, showing me around the RNL facility, training and teaching me, and giving me support for my animal experiments and for radiation delivery to my cells.

To Patrick Ahles, and Pieter Dijkstra, thank you for giving me prompt IT support to smoothen my work. 


\section{To my friends worldwide:}

To Verònica Teixidó Estiarte, you have been like a sister for me, from the day I met you there has always been a special connection and understanding, we have gone through several struggles together, several rocks in the road have made our way tougher but we have always found our way back to each other's lives. I admire your resilient nature, hardworking and trustworthy character, your care for others and your compassion. Our friendship was tried-and-true and I am sure that no matter where we are, our friendship will keep growing and blooming. Love you tons!

To Eva Puerta López, my crib-sis!, who has known me for the longest time and with whom I share a grounded familial friendship. You have seen me literally grow out of old habits and into new ones, you have stayed beside me no matter what life threw our way, and you have shared my passion for singing, dancing, traveling, and helping those in need. May God keep blessing your family and enlighten your way to keep guiding the youngsters you work with. Love you!

To Emilia Schiefthaler, my loving friend since high school, despite the distance and the hectic schedules, every time we speak it seems that time has not gone by, we can easily slip back into our chatty rhythm and keep dancing with our words telling the latest anecdotes of our lives. Our friendship is timeless and treasured. Love you!

To Mckneil Tupaki Rongokavea, thank you for your unwavering affection and support throughout this journey. Whenever we spoke you were always able to joke and make me laugh crazily, so thank you for brightening my day. I relish on the times we spent telling stories and life experiences. You were there for me even when you didn't know that I needed a friend. Your carefree attitude, generosity, and openminded character to accept and learn from new cultures and share yours, makes confiding in you effortless. A heartfelt hug!

To Ajay Sharma and to Ravi Marella, whom I came to know through Kranthi. I have felt always welcome and taken care of at your place and around you, not only by your hospitality and your delicious home-made food, but also by your empathetic and wise nature, with the ability to envision things not so perceptible to the rest of us. Thank you for sharing your knowledge and gentleness with me.

To Benedicta Deogratias, my lovely dancing friend, thank you for the genuine laughs, honesty and comforting words, for the time with you in the dance floor, in 
workshops, celebrating our birthdays or just chatting about human rights, trips or science. I always was very comfortable with you. I hope our friendship keeps flourishing. Big hugs!!

To Jonathan Maël Mélius and to Rio Putra Juni, thank you for your noble friendship and kindhearted character. I cherish the times we spent dancing or chatting, sharing new perspectives of different cultures and ways of living, listening to music, or savoring a home-cooked meal at Eloy's place, your place or mine. Your company was always heart-warming and appreciated. Thank you. Hugs!

To Yusnier Basso Montelier, thank you for the precious times we spent together, your dauntless, caring and comical attitude made time with you a pleasure. I could always rely on you to be there for me, even in the hard times when my daddy, your dear Manolito, passed away. Thank you. Hugs!

To Mohsen Pourpouneh, for your steadfast friendship, your sweet-hearted and supportive nature. I really admired your work ethics which inspired focus and enthusiasm for my $\mathrm{PhD}$. Thank you for always trying to make me happy and for being dependable. I grew fond of our time together and hope that we keep nurturing our friendship over the years. Hugs!

To Carlos Machado Carvajal, María Ramos Canella, María Hernán, Sara Hernán, Víctor Mario Roldán Jaén, Borja García, Ianco Morales, Alfredo Morales, Andrés Iván García, ...you guys have known me throughout all my life, you have beared through my mischievousness and my sweet moments and you have accepted me as I am. Thank you.

To Beatrice, Andrea Burden, Mehrdad Serafi, Agnese Beghè, Enrico Contini, Agata, Patrícia Szép, Kenneth Fyrsterling, Yorick Depour, Anna Zseleva, Kavita Luther, Kamila Joanna, Magda Gromniak, Niken Mahaweni van Ejil,...thank you so much for the amiable and relaxing times together. Our gatherings made time in the Netherlands so much more remarkable. Thank you.

To Efke Tjan, Jeroen Luijer, Marleen van de Laar, Fonny Schouren, Svetlana Podlinova, Saskia, Linda, Claire, Myrurgia Abdul Hamid, ...thank you for the Il Fiore gym training workout you planned for us, for pushing me to work to my maximum potential, and for the vibrating energy and contagious enthusiasm. You magically 
made the stress and worries disappear and you replaced them with fresh and positive thoughts.

To Jacques Rooding, Cedric Roijakkers, Lori Robeers, Janneke den Hartog, Pascal Geusgens, Lieke Klinkenberg, Manon Hoen, Marjolein Caniëls, Stefan, Grace Newton, Alexander Wijnen, Karin, ...thank you all for making the gym classes so much more fun with the war cries: heh!, uoh uoh! and the ay aay ayy's!. You drew smiles in my face and in many other members of the classes. Your effort to fulfill all the exercises planned for us and your motivation are inspiring.

To Brahim Oumou, Edmerd Koffy, Lynn Wouters, and Emmy Mobers thank you for the outstanding hip hop and popping classes at Dansschol Reality or other gyms. Your spontaneity, creativity, and swagger, just made me enjoy myself so much more, especially next to great dancing buddies like Marijke Daems, Sanne Mariani, Benedicta Deogratias, and Lisanne van Dongen.

To Naomi Dongelmans, Khalid Lazar, and Thomas Tcheuffa, thank you for all the salsa, bachata, kizomba, and other Latin dance classes you have organized in Salsa Brisa at Dn Hiemel, La Mulata or Festivals with world renowned dancers performing and teaching workshops. Thank you for the delicious home-made worldly food you prepare for us to savor before the parties, and for the nice chats. Thank you to all my dancing buddies including Hassen Er-Rihani, Marcel Coemaet, Susana Perez, Ricardo Benavides Yabar, ...who like me, feel a passion for dancing and share it with others. The times with you guys were very uplifting. Thank you! 


\section{Curriculum Vitae}




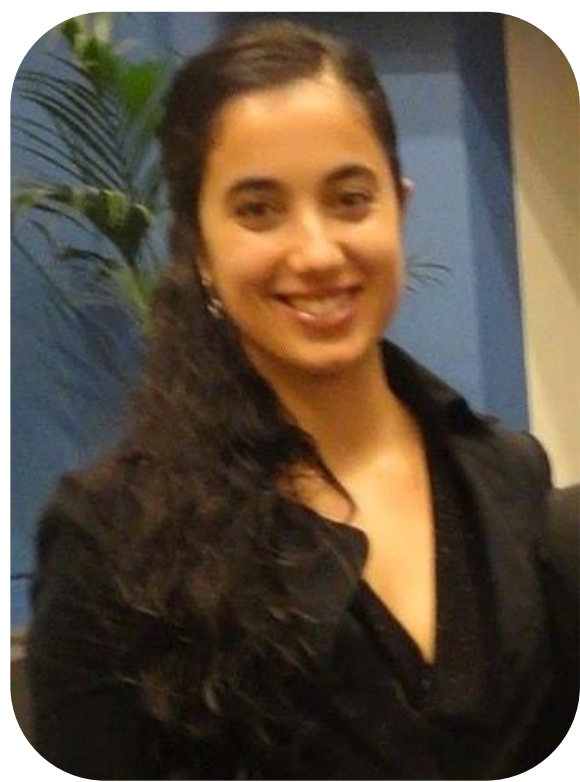

Venus Sosa Iglesias was born on October $8^{\text {th }}$ 1988, in Las Palmas de Gran Canaria, an island of volcanic origin and the second most populated ( 850000 inhabitants) island from the Canarian archipelago located in the Atlantic Ocean, to the west of Africa, but part of Spain. Before the Spanish colonization in 1483 however, the Canarian indigenous inhabitants called it "Tamarán" or "Land of the Brave" and it is considered a "miniature continent" due to the variety of microclimates and landscapes. Throughout the first 17 years of her life, Venus lived in a small village, near the highest mountain in the island (Pico de las Nieves, 1949 meters), named Valsequillo in the inner-eastern area of the island. She attended The American School of Las Palmas and graduated with honors in June 2006. During her high school years, she also attended the Alliance Française in Las Palmas to obtain her A3 certificate in French language. In 2005, she was selected as representative Ambassador of Equatorial Guinea for the Model United Nations (MUN) in the Hague, the Netherlands. The summer of that same year, she did voluntary work in a summer school in her village (Valsequillo) as an English "teacher" for elementary school children.

In September 2006, she started her degree in Biotechnology in the University of Lleida, Catalonia, in the north-eastern part of Spain. She was selected to be Local Coordinator of Research Exchanges for the International Federation of Medical Student's Association (IFMSA) for the University of Lleida. During the summer holidays of 2008 she was granted the opportunity to do a research exchange in Laboratory of Molecular Pathology from the Faculty of Medicine and Dentistry of Palacký University in Olomouc, Czech Republic, in a project entitled "Analysis of the biological role of the tumor suppressor gene PTEN" under the supervision of Dr Alice Hlobilková, MD, PhD. In the summer of 2009, she was granted a second research exchange position in the Immunology Institute of Chung Shan Medical University in Taiwan, in a project entitled "The molecular mechanisms of ornithine decarboxylase-prevented apoptosis in the early events" under the supervision of $\mathrm{Dr}$ Guang Yaw Liu, PhD. In her final two years of her Bachelor degree she did two research projects, both in in the group "Signaling Pathways in Yeast" from the Basic Biomedical Sciences Department in the University of Lleida, the first one on 
"Characterization of the cell surface protein Mtl1 from Saccharomyces cerevisiae" and the second one on "Cellular, Functional and Molecular characterization of the cysteine residues from the MAPK Slt2 from the Cell-Wall Integrity pathway in Saccharomyces cerevisiae" under the supervision of Dr. M ${ }^{a}$ Ángeles de la TorreRuiz. Out of her collaboration with Dr Mima Ivanova Petkova, her work was presented in the "XIX Jornades de Biologia Molecular" (communication \#9) in Barcelona, Spain. In 2011 she started a Master degree in Biomedicine in the University of Barcelona, Spain. Her final Master thesis was in the Oncology and Molecular Pathology Department in Vall d'Hebron Research Institute in Barcelona in a project entitled "microRNAs as possible therapeutic targets for cancer and the linkage between Rplp ribosomal proteins and oxidative stress" under the supervision of Dr. Matilde Esther Lleonart Pajarín.

After her Master's degree, and while in the search for a $\mathrm{PhD}$ position in Oncology, she was a volunteer teacher assistant in the elementary school in Valsequillo where her mother worked in as a teacher. In September 2013 she received a PhD position in the Radiation Oncology Department in the University of Maastricht, The Netherlands, under the supervision of Prof Marc Vooijs, PhD, Ass Prof Jan Theys, $\mathrm{PhD}$, and Ass Prof Ludwig Dubois, $\mathrm{PhD}$, which she completed in 2017. The most relevant results of the thesis are detailed in this book. 


\section{List of Publications}




\section{PUBLISHED}

Sosa V, Moliné T, Somoza R, Paciucci R, Kondoh H, Lleonart ME. Oxidative stress and cancer: an overview. Ageing Res Rev 2013; 12(1):376-90. doi: 10.1016/j.arr.2012.10.004

Yahyanejad S, King H, Iglesias VS, Granton PV, Barbeau LM, van Hoof SJ, Groot AJ, Habets R, Prickaerts J, Chalmers AJ, Eekers DB, Theys J, Short SC, Verhaegen F, Vooijs M. NOTCH blockade combined with radiation therapy and temozolomide prolongs survival of orthotopic glioblastoma. Oncotarget. 2016; 7(27):41251-41264. doi: 10.18632/oncotarget.9275.

\section{IN PREPARATION}

Sosa Iglesias V, Giuranno L, Dubois LJ, Theys J, Vooijs M. Drug resistance in nonsmall cell lung cancer: a potential for NOTCH targeting? Minor revision in Frontiers in Oncology.

Sosa Iglesias V, Theys J, Barbeau L, Lemmens A, Yaromina A, Losen M, Houben R, Groot A, Dubois LJ, Vooijs M. Synergistic enhancement of conventional chemotherapy and chemoradiation in non-small cell lung cancer cell lines by NOTCH/ $\gamma$-secretase inhibition. Minor revision in BMC Cancer.

Sosa Iglesias V, van Hoof SJ, Schyns LEJR, Vaniqui A, Lieuwes N, Yaromina A, Spiegelberg L, Groot AJ, Verhaegen F, Theys J, Dubois LJ, Vooijs M. A novel clinically-relevant orthotopic non-small cell lung cancer model for imageguided small animal radiotherapy platforms. Submitted to British Journal of Radiobiology.

Sosa Iglesias V, Barbeau L, Lieuwes N, Yahyanejad S, Groot AJ, Bussink J, Dubois LJ, Theys J, Vooijs M. NOTCH inhibition may benefit cisplatin and radiation treatment in human NSCLC xenograft models in mice. In preparation. 


\section{POSTERS}

1. “XIX Jornades de Biologia Molecular", Barcelona 2011

2. Wolfsberg meeting, Switzerland 2015

3. ESTRO 3rd FORUM, Barcelona 2015

4. New Advances in animal models and preclinical imaging for translational research in cancerology, France 2015

5. 3rd Symposium in SmART, Ghent 2016

6. European Radiation Research Society, Amsterdam 2016

7. Wolfsberg meeting, Switzerland 2017

\section{ORAL PRESENTATIONS}

1. ESTRO 3rd FORUM, Barcelona, Spain. April 2015

2. GROW Science Day. University of Maastricht. The Netherlands 2015

3. New Advances in animal models and preclinical imaging for translational research in cancerology. La Turballe, France, October 2015

4. 3rd Symposium in Small Animal Precision image-guided radiotherapy. Ghent, Belgium. March 2016

\section{AWARDS}

Travel award and Best participation award for the conference: "New Advances in animal models and preclinical imaging for translational research in cancerology" La Turballe, France, October 2015. 